\title{
energies
}

\section{Biorefinery Based on Olive Biomass}

\section{Edited by \\ Eulogio Castro}

Printed Edition of the Special Issue Published in Energies 


\section{Biorefinery Based on Olive Biomass}





\section{Biorefinery Based on Olive Biomass}

Editor

Eulogio Castro

MDPI $\bullet$ Basel $\bullet$ Beijing $\bullet$ Wuhan $\bullet$ Barcelona $\bullet$ Belgrade $\bullet$ Manchester $\bullet$ Tokyo $\bullet$ Cluj $\bullet$ Tianjin

\section{MDPI}


Editor

Eulogio Castro

Department of Chemical,

Environmental and Materials

Engineering and Center for

Advanced Studies in Energy and Environment,

Universidad de Jaen

Spain

\section{Editorial Office}

MDPI

St. Alban-Anlage 66

4052 Basel, Switzerland

This is a reprint of articles from the Special Issue published online in the open access journal Energies (ISSN 1996-1073) (available at: https://www.mdpi.com/journal/energies/special_issues/ Biorefinery_Biomass\#).

For citation purposes, cite each article independently as indicated on the article page online and as indicated below:

LastName, A.A.; LastName, B.B.; LastName, C.C. Article Title. Journal Name Year, Article Number, Page Range.

ISBN 978-3-03943-561-6 (Hbk)

ISBN 978-3-03943-562-3 (PDF)

Cover image courtesy of Eulogio Castro.

(c) 2020 by the authors. Articles in this book are Open Access and distributed under the Creative Commons Attribution (CC BY) license, which allows users to download, copy and build upon published articles, as long as the author and publisher are properly credited, which ensures maximum dissemination and a wider impact of our publications.

The book as a whole is distributed by MDPI under the terms and conditions of the Creative Commons license CC BY-NC-ND. 


\section{Contents}

About the Editor $\ldots \ldots \ldots \ldots \ldots \ldots \ldots \ldots \ldots \ldots \ldots$

Preface to "Biorefinery Based on Olive Biomass" $\ldots \ldots \ldots \ldots \ldots \ldots \ldots$ ix

José Luis Fernández, Felicia Sáez, Eulogio Castro, Paloma Manzanares, Mercedes Ballesteros and María José Negro

Determination of the Lignocellulosic Components of Olive Tree Pruning Biomass by Near Infrared Spectroscopy

Reprinted from: Energies 2019, 12, 2497, doi:10.3390/en12132497 . . . . . . . . . . . . . . .

José Carlos Martínez-Patiño, Irene Gómez-Cruz, Inmaculada Romero, Beatriz Gullón, Encarnación Ruiz, Mladen Brnčićc and Eulogio Castro

Ultrasound-Assisted Extraction as a First Step in a Biorefinery Strategy for Valorisation of Extracted Olive Pomace

Reprinted from: Energies 2019, 12, 2679, doi:10.3390/en12142679 . . . . . . . . . . . . . . 11

Amani Taamalli, Jesus Lozano Sánchez, Haifa Jebabli, Najla Trabelsi, Leila Abaza, Antonio Segura Carretero, Jae Youl Cho and David Arráez Román

Monitoring the Bioactive Compounds Status in Olea europaea According to Collecting Period and Drying Conditions

Reprinted from: Energies 2019, 12, 947, doi:10.3390/en12050947 .

Antonio Lama-Muñoz, María del Mar Contreras, Francisco Espínola, Manuel Moya, Inmaculada Romero and Eulogio Castro

Optimization of Oleuropein and Luteolin-7-O-Glucoside Extraction from Olive Leaves by Ultrasound-Assisted Technology

Reprinted from: Energies 2019, 12, 2486, doi:10.3390/en12132486 . . . . . . . . . . . . . . . . .

Markella Tzirita, Maria Kremmyda, Dimitris Sarris, Apostolis A. Koutinas and Seraphim Papanikolaou

Effect of Salt Addition upon the Production of Metabolic Compounds by Yarrowia lipolytica Cultivated on Biodiesel-Derived Glycerol Diluted with Olive-Mill Wastewaters

Reprinted from: Energies 2019, 12, 3649, doi:10.3390/en12193649 . . . . . . . . . . . . . . .

Antonio Serrano, Fernando G. Fermoso, Bernabé Alonso-Fariñas, Guillermo Rodríguez-Gutiérrez, Sergio López, Juan Fernandez-Bolaños and Rafael Borja Long-Term Evaluation of Mesophilic Semi-Continuous Anaerobic Digestion of Olive Mill Solid Waste Pretreated with Steam-Explosion Reprinted from: Energies 2019, 12, 2222, doi:10.3390/en12112222 . 



\section{About the Editor}

Eulogio Castro has a Ph.D. in Chemical Engineering from the University of Granada, Spain, where he also worked as a teacher in undergraduate and graduate studies in Chemistry and Chemical Engineering. Since the foundation of the University of Jaén in 1993, he has served as a teacher and researcher, and he is currently a Full Professor of Chemical Engineering. He was also an Invited Researcher at the Institute of Biotechnology in UNAM, Mexico as well as at the École Nationale Supérieure de Chimie de Toulouse, France, and a Visiting Assistant Professor at the University of Florida, USA.

His main research interests are focused on the conversion of biomass into added-value products. Different agricultural and agroindustrial materials have been used as raw materials for the development of the biorefinery concept, including sugarcane bagasse, rapeseed straw, brewer spent grains, sunflower stalks and especially all the by-products from olive tree cultivation and those derived from the olive oil production process. Prof. Castro has studied the products, biofuels, renewable chemicals and biomaterials as well as the techno-economic and environmental issues related to biorefinery deployment. 



\section{Preface to "Biorefinery Based on Olive Biomass"}

Olive tree cultivation is attracting increasing interest worldwide due to the health benefits of olive oil consumption. Currently, more than eleven million hectares of olive trees are spread across sixty countries, with those in the Mediterranean basin being the main olive oil producers. As a consequence of the production of olive oil, a huge amount of biomass is generated yearly, coming from two different origins. On the one hand, the pruning of olive trees produces a great deal of biomass that must be eliminated from cultivation fields. On the other hand, the olive oil production process is also responsible for the generation of a number of biomasses, such as olive leaves, pits, pomace and even olive-mill wastewater, all of which must be adequately handled. All these biomasses can be exploited as raw materials for the production of a number of biocompounds under the biorefinery scheme. This Special Issue of Bioenergies aims to summarize the recent research carried out about these different biomasses, along with procedures, methods and configurations to obtain a wide range of bioproducts, including but not limited to biofuels, lignin, natural antioxidants, bio-based compounds, fermentable sugars, etc. In addition to the environmental advantages derived from avoiding the usual practices of disposal of such residues (now referred to as by-products), the economic relevance of this proposal is undeniable, even if further research efforts must be accomplished.

The accepted papers deal with virtually all the stages in the exploitation of olive-derived biomass as a feedstock for a biorefinery, from the characterization of the chemical composition (including the bioactive compounds) to the treatment of the final by-products obtained after olive oil production, such as olive-mill wastewater, olive solid residues or even the extracted olive pomace.

This collection of scientific articles is an excellent source of information that can be used as a starting point to develop a further business model beyond the conventional and limited just-olive-oil-producing facility.

I would like to thank the professional staff of MDPI for their efforts that made the Special Issue and this edition possible.

Eulogio Castro

Editor 



\title{
Determination of the Lignocellulosic Components of Olive Tree Pruning Biomass by Near Infrared Spectroscopy
}

\author{
José Luis Fernández ${ }^{1}$, Felicia Sáez ${ }^{1}$, Eulogio Castro ${ }^{2}$, Paloma Manzanares ${ }^{1}$, \\ Mercedes Ballesteros ${ }^{1}$ and María José Negro ${ }^{1, *}$ \\ 1 Biofuels Unit, Energy Department, Research Centre for Energy, Environment and Technology (CIEMAT), \\ Complutense Av, 22, 28040 Madrid, Spain \\ 2 Department of Chemical, Environmental and Materials Engineering, University of Jaén, \\ Campus Las Lagunillas, 23071 Jaén, Spain \\ * Correspondence: mariajose.negro@ciemat.es
}

Received: 30 May 2019; Accepted: 26 June 2019; Published: 28 June 2019

\begin{abstract}
The determination of chemical composition of lignocellulose biomass by wet chemistry analysis is labor-intensive, expensive, and time consuming. Near infrared (NIR) spectroscopy coupled with multivariate calibration offers a rapid and no-destructive alternative method. The objective of this work is to develop a NIR calibration model for olive tree lignocellulosic biomass as a rapid tool and alternative method for chemical characterization of olive tree pruning over current wet methods. In this study, 79 milled olive tree pruning samples were analyzed for extractives, lignin, cellulose, hemicellulose, and ash content. These samples were scanned by reflectance diffuse near infrared techniques and a predictive model based on partial least squares (PLS) multivariate calibration method was developed. Five parameters were calibrated: Lignin, cellulose, hemicellulose, ash, and extractives. NIR models obtained were able to predict main components composition with $\mathrm{R}_{\mathrm{cv}}^{2}$ values over 0.5 , except for lignin which showed lowest prediction accuracy.
\end{abstract}

Keywords: lignocellulosic components; feedstock analysis; near-infrared spectroscopy; olive tree pruning

\section{Introduction}

Within the last two decades, components of biomass materials such as lignocellulosic residues have increasingly received more attention in the science community due to their potential for the production of biofuels, as well as new value-added compounds and biomaterials utilizing a "biorefinery" approach.

Olive trees are usually native to the Mediterranean countries, but cultivation has spread globally during the past two decades due to healthy benefits attributed to olive oil consumption. Currently, olive trees are cultivated in more than 40 countries, and the total dedicated surface is about 10.8 million ha in 2017 [1]. Olive tree pruning (OTP) biomass takes place immediately after fruit collection and is an essential operation performed every two years after fruit harvesting. In the Mediterranean region, residual biomass from olive tree pruning yield ranges from 1 to 5 and from 4 to 11 oven dry tha ${ }^{-1}$ respectively for Spanish and Italian orchards [2]. Older branches are cut down, gathered into to the center of each row of trees, and further treated. This agricultural residue must be promptly eliminated from the cultivation fields; otherwise a risk for vegetal diseases may arise. Currently, two different applications for this pruning biomass exist, either grinding it or scattering the chips over the field or direct burning. Direct burning represents the most common method of disposal, which puts the field at risk of an uncontrollable fire and thus an economic cost, although some initiatives are being carried out to develop collecting techniques [3]. It has been proposed to use this waste as raw material for 
obtaining a broad range of products [4,5], including energy, biofuels as bioethanol [6-9], antioxidant compounds [10,11], oligosaccharides [12], and others [13].

For any application of olive tree pruning residue it is essential to know its chemical composition in order to establish the most adequate conversion process. Moreover, the ratio of main structural components i.e., cellulose, hemicellulose, and lignin, as well as other minor components in a particular biomass, is of crucial importance to determine the potential markets applications. The current methods of chemical characterization of the composition of biomass feedstock are labor-intensive and time consuming, and thus do not meet industrial requirements for providing quick measurements. Moreover, they are often based on wet-laboratory analysis, during which the integrity and structure of the material is destroyed [14]. By contrast, the non-destructive spectroscopy techniques provides analytical information without damaging the sample and in most of the cases, in addition, sample treatment is not required. Vibrational techniques such as IR spectroscopy are amongst the non-destructive spectroscopy techniques that have attracted more interest in the last years [15,16]. Additionally, infrared spectroscopy techniques are fast, accurate, and low-cost analytical methods with high potential in biomass composition analysis [17,18]. In this context, diffuse reflectance near infrared spectroscopy (NIR) has been suggested as a re- and non-destructive method to replace reference methods for determination of lignocellulosic components of feedstock and capacity for bioethanol production [19]. In fact, near-infrared spectroscopic scanning (NIR) coupled with multivariate calibration methods have been developed to characterize different herbaceous feedstocks [20], switchgrass [21], pine [22], yellow-poplar [23], willow [24], Miscanthus [25], and bamboo [26]. NIR technique application for olive residues has been reported to analyze solid fuels for heat and power generation [27]. However, to date, there is no literature on NIR application to determine chemical composition of olive tree pruning biomass for liquid biofuels such as bioethanol or other valuable products.

This work attempts to demonstrate the effectiveness of near infrared diffuse reflectance spectroscopy (NIR) as a rapid and non-destructive method as an alternative to the wet chemical analysis methods for the determination of structural carbohydrates, lignin, and ash in olive tree pruning biomass.

\section{Materials and Methods}

\subsection{Raw Material}

Olive tree pruning was collected from different locations in Andalusia (Spain) after fruit harvesting. A total of 79 samples were gathered, 64 of which were used for calibration models, and 15 for external validation. Samples used in this work were collected manually, using a pruning shear, and corresponded to shoots less than $3 \mathrm{~cm}$ diameter with approximately $70 \%$ of leaves and $30 \%$ thin steams by weight. All samples were air-dried at room temperature to equilibrium moisture content of about $10 \%$ and milled using a centrifugal mill (Retsch ZM200, Retsch, Ins., Haan, Germany) to $2 \mathrm{~mm}$ particle size. A sub-sample of milled olive tree pruning was used for analysis of chemical composition (cellulose, hemicellulose, total lignin, extractives, and ash) and another sub-sample for NIR spectroscopy.

\subsection{Wet Chemical Composition Analysis}

Chemical composition of the olive tree pruning was determined according to (National Renewable Energy Laboratory (NREL) procedures for biomass composition analysis [28]. Firstly, extractives content was determined by Dionex Accelerated Solvent Extractor System (ASE 200). Extraction was performed in water and ethanol. After extraction, cellulose and hemicellulose content was measured based on monomers content after a two-step acid hydrolysis procedure to fractionate the fiber. A first step with $72 \%(\mathrm{w} / \mathrm{w})$ sulphuric acid at $30{ }^{\circ} \mathrm{C}$ for $60 \mathrm{~min}$ was used, followed by a second step in which the reaction mixture was diluted to $4 \%$ sulphuric acid and autoclaved at $121{ }^{\circ} \mathrm{C}$ for $1 \mathrm{~h}$. Sugars concentration was determined by high-performance liquid chromatography (HPLC) in a Waters 2695 liquid chromatograph with refractive index detector. A CARBOSep CHO-682 LEAD column 
(Transgenomic, Omaha, NE, USA) operating at $75{ }^{\circ} \mathrm{C}$ with Milli-Q water (Millipore Corporation, Billerica, MA, USA) as mobile-phase $(0.5 \mathrm{~mL} / \mathrm{min})$ was used. Anhydrous correction was applied to the quantification results of monomeric sugars to calculate the polymer of carbohydrates. The factor used to convert sugars monomers to anhydromonomers were 0.90 for glucose to glucan, galactose to galactan, mannose to mannan and 0.88 for xylose to xylan, arabinose to arabinan. Hemicellulose was calculated as the sum of xylan, arabinan, galactan, and mannan concentrations. Total lignin was calculated as the sum of acid soluble and acid insoluble lignin. All measurements were done in triplicate and results are presented as percentage on an oven-dry weight basis. Results from wet chemical analysis were used for calibration and validation of the near infrared method.

\subsection{NIR Spectroscopy}

A total of 79 samples of whole biomass samples, ground to $2 \mathrm{~mm}$ particle size, were analyzed. The biomass samples were dried in oven at $40{ }^{\circ} \mathrm{C}$ for $48 \mathrm{~h}$ before testing. NIR spectra were measured in spinning Petri dishes, using a Perkin Elmer NIR Spectrometer; model Spectrum One NTS (Perkin Elmer Inc., Beaconsfield, UK), with diffuse reflectance accessory.

The spectra were collected by averaging 70 scans, six spectra were measured, and a mean spectrum of each sample was calculated for the construction of the predictive models. The spectral range selected for analysis was 10,000 to $4000 \mathrm{~cm}^{-1}$ and spectral resolution of $8 \mathrm{~cm}^{-1}$.

\subsection{Development of NIR Calibration}

The spectral and wet chemical data were processed with Spectrum Quant+ software, version 4.51 (Perkin Elmer Inc., Billerica, MA, USA) for chemometric analysis. This includes reduction of number of variables by principal component analysis (PCA) and multiple regressions. A partial least square (PLS) multivariate calibration model was developed. To improve the correlation between the spectra and concentration data, a number of preprocessing techniques for spectral data have been utilized. To minimize baseline deviations caused by dispersion effect, the smoothing according to Savitzky-Golay algorithm [29] has been applied. The first and second derivatives were applied to remove additive and sloped baseline drifts, and finally, normalization methods like multiplicative signal correction (MSC) and standard normal variate (SNV) remove the multiplicative signal effects produced by differences of particle size in samples. The two pre-processing methods can also be combined, first and second derivative with SNV or MSC, to obtain better results. All of these pretreatments were tested.

\subsection{Analysis and Validation of Calibration Models}

The application of PLS process allows building a linear regression model based on the relation between the spectral data matrix and analyte concentration matrix. The calibration results were assessed by statistic parameters, which decide how adequately the calibration fits the data and how adequately the calibration will predict external samples. The root mean square error of calibration (RMSEC) parameter was used to evaluate the calibration and it is a statistical term that measures the standard deviation of residuals (differences between observed and predicted values) in the regression equation and measures precision of fit between data and the calibration model.

The model validation in the present study included both full-cross validation (leave-one-out) and independent validation. Cross-validation (leave-one-out method, LOO) was used as a basis to calculate the optimal range of principal components and compare the prediction ability of different calibration models. In full cross-validation procedure, for a dataset on $\mathrm{n}$ samples, one sample is left out and the rest of (n-1) samples are calibrated using PLS to generate a subset model. The full cross-validation results were expressed using the term root mean square error of cross-validation (RMSECV), and cross-validation coefficient of determination $\left(\mathrm{R}^{2}{ }_{\mathrm{cv}}\right)$. On the other hand, 15 samples were selected randomly from the entire set, to be used for an external validation of the best calibration models chosen by the cross validation criterion. The result of the external validation is defined by the 
statistic parameter root mean square error of prediction (RMSEP) and external validation coefficient of determination $\left(\mathrm{R}^{2}\right.$ ext $)$.

A key factor in determining the quality of a model is the optimum number of principal components (PCs) (or PLS factors) to include in the model. Too many PCs results in overfitting and too few PCs result in a low accurate model. There are various criteria to select the appropriate number of PCs; based on the variation of the eigenvalues with the number of PCs [30]; based on minimum value of RMSECV or an optimal RMSECV value that uses the significance F-test, according to Haaland and Thomas criteria [31]. In this work, the latter criterion was chosen.

\section{Results and Discussion}

\subsection{Wet Chemical Composition}

The chemical composition of olive pruning biomass is presented in Table 1. Results include mean value of composition, standard deviation (SD), coefficient of variation (CV), and concentration range.

In OTP biomass, cellulose content is in the range of $8.6-19.8 \%(w / w)$. Hemicellulose, lignin content, and ash content from OTP samples are in the range of $9.4-16.3 \%, 15.0-19.9 \%$, and $5.1-10.0 \%$ $(\mathrm{w} / \mathrm{w})$, respectively. It is worthwhile to mention that this lignocellulosic residue has an extractive content close to $40 \%$ (range $32.5-46.7 \%, \mathrm{w} / \mathrm{w}$ ). The coefficient of variation (CV) expresses the variation as a percentage of the mean and is often preferred as a measure of data dispersion (SD), since SD increases in proportion to concentration values. It is important to notice the low value of the coefficient of variation for lignin and extractives. The compositional variance is a factor to consider in order to achieve robust models.

The proportion of the extractive fraction was greater than that reported for olive tree pruning; extractive contents ranged from $23.3 \%(w / w)$ [32] to $31.4 \%(w / w)$ [33]. The variability of composition was attributed mainly to the heterogeneity of the residue (changing proportions of small branches and leaves). The high proportion of extractives could be related to a higher content of leaves in the original samples; high content in extractives in olive leaves (38.8\% dry weight) have been reported by other authors [34].

The summative mass closure gives a value of $88.3 \%$; the remaining percentage $(11.7 \%)$ may be attributed to other minor compounds such as acetyl groups (from hemicellulose fraction), crude protein, and other unanalyzed components, like pectins.

Table 1. Summary of chemical composition of calibration set. All data $(\% \mathrm{w} / \mathrm{w})$ are on a moisture-free basis.

\begin{tabular}{cccccc}
\hline Components & Extractives & Cellulose & Hemicellulose & Lignin & Ash \\
\hline Mean & 40.0 & 12.0 & 12.0 & 17.4 & 7.0 \\
SD & 3.0 & 2.1 & 1.7 & 1.1 & 1.1 \\
CV & 7.5 & 17.5 & 13.9 & 6.4 & 15.2 \\
Range & $32.5-46.7$ & $8.6-19.8$ & $9.4-16.3$ & $15.0-19.9$ & $5.1-10.0$ \\
\hline
\end{tabular}

\subsection{NIR Calibration}

\subsubsection{Selection of Wavenumber Regions}

The NIR radiation covers a wavelength range between 750 and $2500 \mathrm{~nm}$ (13,000 to $4000 \mathrm{~cm}^{-1}$ in wavenumber units). This range includes first, second, third overtones, and a combination of the fundamental bands. As the spectral information is redundant, the wavenumber selection can improve the robustness of multivariate calibration models if the right choices are made. The use of derivative filters was explored as a tool for spectral resolution enhancement. A set of reflectance spectra (expressed in absorbance unities vs. wavenumber) of olive tree pruning samples are shown in Figure bands between $10,000 \mathrm{~cm}^{-1}$ and $7500 \mathrm{~cm}^{-1}$ have been associated with third overtones and their low intensity and excessive noise makes them less suitable to calibration process ( $\mathrm{He}$ and $\mathrm{Hu}, 2013$ ). 
The greatest variability between spectra is observed in the range $7500-4200 \mathrm{~cm}^{-1}$. We can observe this variability in first derivative spectra represented in Figure 1B. There are three interesting zones: 7100 to $6900 \mathrm{~cm}^{-1}, 6000$ to $5600 \mathrm{~cm}^{-1}$ (associated with lignin and extractives first overtones bands), and the region between 5500 and $4000 \mathrm{~cm}^{-1}$. The latter includes the main band of water at approximately $5200 \mathrm{~cm}^{-1}$ and combination bands associated with stretching vibrations of $\mathrm{CH}, \mathrm{CH}_{2}$, and $\mathrm{CH}_{3}$ bonds of carbohydrates, although the spectral noise in this range is high. In Figure 1C, we can observe the second derivative. There are two well-resolved bands in the second derivative associated with phenolic compounds at 6900 and $5980 \mathrm{~cm}^{-1}$. However, care should be taken when choosing the wavenumber range, in order to avoid a loss of information. A reduced wavenumber region $\left(7500-5500 \mathrm{~cm}^{-1)}\right.$ was also selected to create calibration models and compare the results with those obtained from the full spectral range studies. This range mainly includes the first overtone bands, corresponding to $\mathrm{OH}$ stretching vibrations associated with carbohydrates and phenolic groups, and $\mathrm{CH}$ stretching vibrations of both aliphatic and aromatic bonds [35]. The reduced spectral range excludes water principal band extending between 5200 and $5000 \mathrm{~cm}^{-1}$, which consists of multiple overlapping bands. The apparent location of these bands changes as the spectra are measured from one to another.
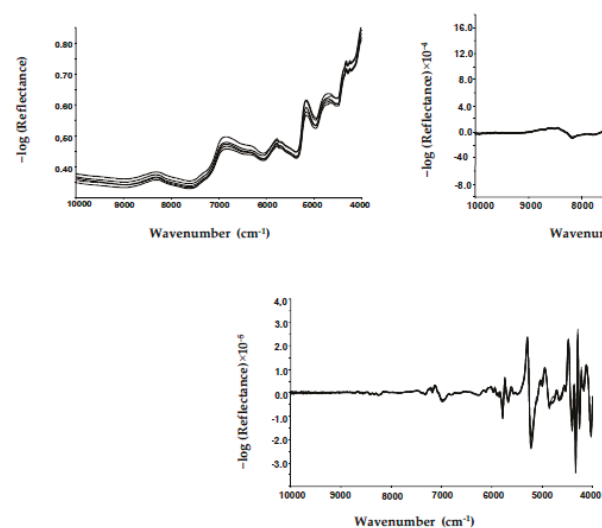

C
B

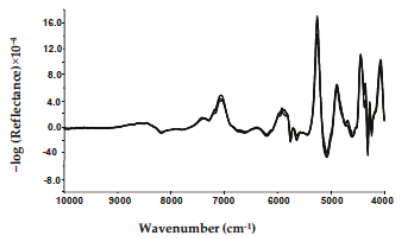

Figure 1. Near infrared spectra of olive tree pruning samples: (A) Raw spectra, (B) first derivative spectra; and (C) second derivative spectra.

\subsubsection{NIR Calibration Development}

PLS regression was performed on the near infrared spectra by using spectral preprocessing methods and using different spectra ranges. Results of the best prediction models of chemical components of OTP using full spectra $\left(10,000-4000 \mathrm{~cm}^{-1}\right)$ and selected spectra region $\left(7500-5500 \mathrm{~cm}^{-1}\right)$, respectively, are presented in Table 2. The results show how the calibration quality varies with the wavenumber range and spectral mathematical treatments. In all cases, the application of Savitzky-Golay derivation with smoothing step (five points) is essential to reach good correlations. The MSC and SNV were compared as normalization methods (either alone or in combination with first derivative filter). The two preprocessing methods gave similar results.

Regarding cellulose and extractives content, the models based on the restricted wavenumber $7500-5500 \mathrm{~cm}^{-1}$ gave the best results using first derivative with SNV for cellulose and first derivative with SNV as pretreatment for extractives. The coefficient of determination for calibration $\left(\mathrm{R}^{2}\right.$ cal $)$ indicates a good linear fit (0.95 for cellulose and 0.91 for extractives), while lower errors of prediction (RMSECV) were found for the restricted range model: 1.75 for extractives and 0.94 for cellulose. 
The coefficients of determination $\left(\mathrm{R}_{\mathrm{cv}}^{2}\right)$ also give the best results in restricted-range models $(0.80$ for cellulose and 0.65 for extractives).

For hemicellulose and lignin content, the models based on the restricted wavenumber $7500-5500 \mathrm{~cm}^{-1}$ gave similar results to those obtained with full spectra range, which indicates that reduction of wavenumber range does not improve the calibration results. For hemicellulose content, the statistics of fit were also equivalent ( 0.72 and 0.73$)$. The same is true for validation parameters, RMSECV give 0.88 and $R^{2}{ }_{c v} 0.72$ for reduced range and 0.89 and 0.71 for full spectrum, respectively.

For total lignin, although the $\mathrm{R}^{2}$ cal values (0.56) indicate some degree of fit, it is remarkable the relatively low correlation in all models studied and the rapid increase of prediction errors when introduce a greater number of principal components in model. The data pretreatment in the two wavenumber ranges (full and restricted) was the second derivative. Good fits with low number of PCs are achieved, but the prediction quality becomes noticeably worse and RMSECV values are higher than the other calibration models.

Organic nitrogenous compounds present in this agricultural waste biomass, in addition to an insufficient organic nitrogenous compounds extraction, can cause interference with the wet chemical characterization method used [36]. The interference with the acid insoluble lignin values caused by the presence of organic nitrogen compounds has been explained by the Maillard reactions. Indeed, with the wet chemical method utilized, during the second hydrolysis step $\left(121^{\circ} \mathrm{C}, 1 \mathrm{~h}\right)$, a fraction of the organic nitrogen compounds that was not removed during extraction step, could react with sugars produced during cellulose and hemicellulose, forming insoluble substances that could cause an overestimation of the lignin values.

For ash content, prediction models for OTP developed with the full spectra regions exhibited the best correlation values $\left(\mathrm{R}^{2}\right.$ cal of 0.96$)$. The RMSECV $(0.52)$, clearly indicate a best predictive power compared to reduced spectral range models $(0.74)$.

Table 2. Results of PLS1 calibration and prediction models developed for chemical composition of olive pruning biomass $(\% \mathrm{w} / \mathrm{w})$.

\begin{tabular}{|c|c|c|c|c|c|c|c|c|c|}
\hline Components & $\begin{array}{l}\text { Wavenumber } \\
\left(\mathrm{cm}^{-1}\right)\end{array}$ & $\begin{array}{c}\text { Data } \\
\text { Preprocessing }\end{array}$ & PCs & $\mathbf{R}_{\text {cal }}^{2}$ & RMSEC & $\mathbf{R}^{2} \mathrm{cv}$ & RMSECV & $\mathbf{R}^{2}$ ext & RMSEP \\
\hline \multirow{2}{*}{ Extractives } & $7500-5500$ & 1st derivative & 5 & 0.91 & 0.93 & 0.65 & 1.75 & 0.79 & 2.33 \\
\hline & $10,000-4000$ & 1st der + SNV & 4 & 0.82 & 1.30 & 0.45 & 2.05 & 0.69 & 2.25 \\
\hline \multirow{2}{*}{ Cellulose } & $7500-5500$ & 1st der $+\mathrm{SNV}$ & 5 & 0.95 & 0.51 & 0.80 & 0.94 & 0.84 & 1.06 \\
\hline & $10,000-4000$ & 1st derivative & 3 & 0.83 & 0.89 & 0.73 & 1.08 & 0.70 & 1.75 \\
\hline \multirow{2}{*}{ Hemicellulose } & $7500-5500$ & 1st der + SNV & 3 & 0.82 & 0.72 & 0.72 & 0.88 & 0.87 & 0.84 \\
\hline & $10,000-4000$ & 1st derivative & 3 & 0.82 & 0.73 & 0.71 & 0.89 & 0.83 & 0.95 \\
\hline \multirow{2}{*}{ Lignin } & $7500-5500$ & 2nd derivative & 1 & 0.56 & 0.75 & 0.05 & 1.08 & 0.29 & 1.00 \\
\hline & $10,000-4000$ & 2nd derivative & 1 & 0.56 & 0.75 & 0.01 & 1.11 & 0.04 & 1.38 \\
\hline \multirow{2}{*}{ Ash } & $7500-5500$ & 1st derivative & 5 & 0.85 & 0.42 & 0.52 & 0.74 & 0.85 & 0.80 \\
\hline & $10,000-4000$ & 1st der + SNV & 6 & 0.96 & 0.21 & 0.76 & 0.52 & 0.85 & 0.51 \\
\hline
\end{tabular}

The predictive power data represented in Table 2 give poor results in some cases. There are various factors that account for these results. First, the variability of the biomass composition data, expressed as standard deviation of the mean or coefficient of variation, was particularly low in the case of hemicellulose and lignin. The calibration and prediction results obtained are similar to those tested by other authors on biomass feedstocks, as yellow poplar [23], Miscanthus [25], or a mixture of biomasses [21]. The relatively poor values of calibration and external validation statistics associated with lignin have been also described for other complex biomass such as Eucalyptus [37].

Figure 2 provides a graphical representation of the prediction ability for the five parameters according to cross validation and external samples analysis. 


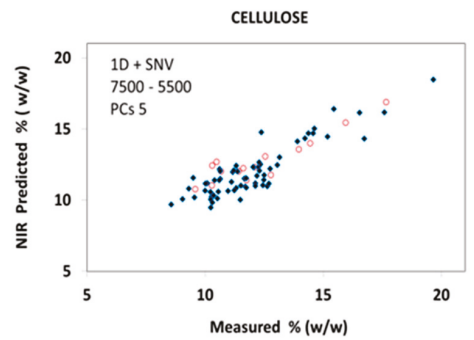

(a)

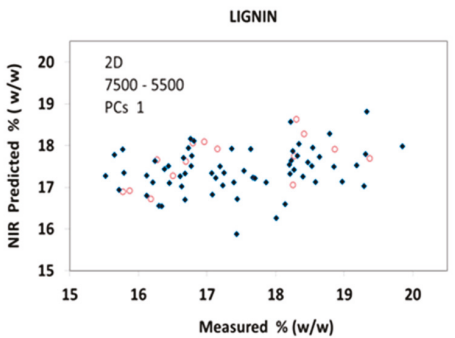

(c)

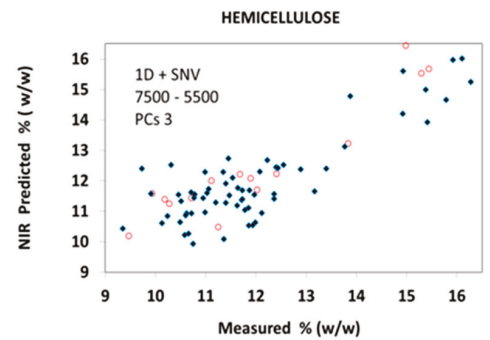

(b)

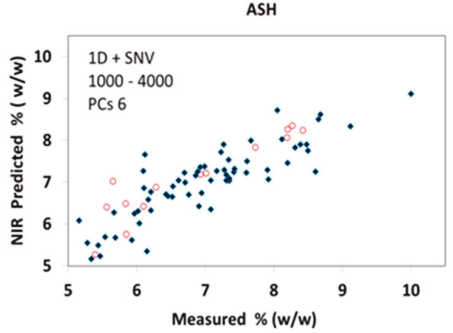

(d)

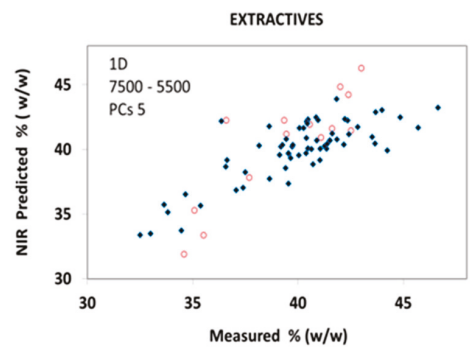

(e)

Figure 2. Near infrared (NIR)-predicted vs. measured plot of cellulose (a), hemicellulose (b), lignin (c), ash (d) and extractives (e) content in olive tree pruning: $(\downarrow)$ cross validation and $(\bigcirc)$ external validation.

\subsubsection{External Validation of Model}

The 15 samples not included in calibration model were used for external validation. These samples were chosen so that the mean and standard deviation are very similar to the calibration samples. Figure 2 shows also the prediction results for cellulose, hemicellulose, lignin, ash, and extractives corresponding to 15 samples of OTP according to the best model for each parameter depicted in Table 2. The correlation between predicted composition and measured composition is based on the optimal calibration applied over every analyte (Table 2). The predictive ability of the model to these external samples is defined in this work by the root mean square error of prediction (RMSEP), which is a measure of the variability of the difference between the predicted and reference values for a set of validation samples. The RMSEP values are shown in Table 2, and the RMSEP values found for the five parameters for whole range wavenumber method are: 1.75 for cellulose, 0.95 for hemicellulose, 2.25 for extractives, 1.38 for lignin, and 0.51 for ash. This RMSEP values are comparable to cross-validation values, although in the case of cellulose, the RMSEP value is about 60 percent larger than the RMSECV one. For the models constructed using reduced spectral range, RMSEP give the following values: Extractives 2.33, cellulose 1.06, hemicellulose 0.84, lignin 1.00, and ash 0.80 . Nevertheless, coefficient of determination $\left(\mathrm{R}^{2}\right.$ ext $)$ for external validation shows higher values than cross-validation coefficients. 
As a summary of the discussion above, it can be stated that the analyses of statistical data collected give an idea of the predictive capacity of the models. The RMSEC values indicate a good fit, but RMSECV values denote only discrete ability of prediction. Some other criteria are also available for this purpose. There are references about 0.5 as a threshold value of $\mathrm{R}_{\mathrm{cv}}^{2}$ in $\mathrm{LOO}$ cross validation; values over 0.5 are indicative of good prediction capacity [38], although it is true that LOO procedure utility as goodness of prediction has been questioned [39]. But, in any case, the calibration models of this work have values over 0.5 except for lignin. It is not easy to explain this poor behavior for lignin. On the one hand, it must be pointed out that the high content in extractives of this biomass may be interfering with spectral data analysis associated with lignin, only partly resolved applying the second derivative. On the other hand, it is important to consider that the total lignin content value comprises both the insoluble and the acid soluble lignin, and the later measurement method may imply errors associated with the use of an absorptivity coefficient not specifically determined for OTP. Moreover, the acid insoluble lignin could be overestimated by using the wet chemical analysis due to condensation reaction of relatively high concentration of protein found in OTP samples (around 8\%,w/w) [40].

For ash content measurement, previous studies have shown that is possible to determine inorganic compounds using NIR spectroscopy [41]. Simple inorganic constituents will not absorb directly in NIR region, and the ash content was indirectly determined through correlation with NIR absorbing organic compounds. The influence of inorganic elements on organic compounds NIR bands is expected to occur over full spectrum and not only in the NIR range of $7500-5500 \mathrm{~cm}^{-1}$. The monatomic ions can be coordinated with $\mathrm{C}-\mathrm{H}, \mathrm{N}-\mathrm{H}$, and $\mathrm{O}-\mathrm{H}$ bonds, which produce absorption bands across the whole NIR spectrum.

Finally, the comparison between coefficients of determination calculated for the two methods of validation (internal and external), reflects a best fit of the external sample set, but taking into account the relatively low number of samples available, the use of cross validation as a reference evaluation of calibration models is justified.

\section{Conclusions}

Results show that near infrared spectroscopy combined with multivariate calibration can be useful as a predictive technique for olive tree pruning biomass analysis, despite its specific compositional features characterized by its high content and variability of the extractives fraction. The predictability of the models in this study is, in principle, limited for the low number of available samples and low compositional variability of calibration set. In spite of this, the biomass components analyzed and predicted with the best model exhibit $\mathrm{R}^{2}$ cal values of 0.9 or near of 0.9 , and acceptable values of prediction errors. The exception was total lignin, which exhibit a poor linear fit $\left(R_{\text {cal }}^{2}<0.6\right)$ and greater prediction errors. Thus, further work is needed in order to improve its potential as a prediction tool and this implies that to increase the number and compositional variability of the sample set, both calibration and external validation are needed.

Author Contributions: Conceptualization, M.J.N. and E.C.; methodology, M.J.N., J.L.F.; validation, J.L.F.; formal analysis, F.S.; investigation, M.J.N., M.B., F.S., J.L.F.; writing—original draft preparation, M.J.N., P.M.; writing—review and editing, P.M., M.J.N.

Funding: This research was partially funded by Ministerio de Economía y Competitividad (MINECO) (Spain) Reference ENE2011-29112-C02-01 and ENE2011-29112-C02-02 including FEDER funds and EN2017-85819-C2-2-R (AEI/UE).

Acknowledgments: Authors would like to thank to MINECO (Spain) Reference ENE2011-29112 including FEDER funds.

Conflicts of Interest: The authors declare no conflict of interest.

\section{References}

1. Faostat. Available online: http://www.fao.org/faostat/en/\#home (accessed on 19 February 2019). 
2. Spinelli, R.; Picchi, G. Industrial harvesting of olive tree pruning residue for energy biomass. Bioresour. Technol. 2010, 101, 730-735. [CrossRef] [PubMed]

3. Pari, L.; Suardi, A.; Frąckowak, P.; Adamczyk, F.; Szaroleta, M.; Santangelo, E.; Bergonzoli, S.; Del Giudice, A.; Dyjakon, A. Two innovative prototypes for collecting pruning biomass: Early performance tests and assessment of the work quality. Biomass Bioenergy 2018, 117, 96-101. [CrossRef]

4. Romero-García, J.M.; Niño, L.; Martínez-Patiño, C.; Álvarez, C.; Castro, E.; Negro, M.J. Biorefinery based on olive biomass. State of the art and future trends. Bioresour. Technol. 2014, 159, 421-432. [CrossRef] [PubMed]

5. Negro, M.J.; Manzanares, P.; Ruiz, E.; Castro, E.; Ballesteros, M. The biorefinery concept for the industrial valorization of residues from olive oil industry. In Olive Mill Waste; Galanakis, C.M., Ed.; Academic Press: London, UK, 2017; pp. 57-78.

6. $\quad$ Manzanares, P.; Negro, M.J.; Oliva, J.M.; Saéz, F.; Ballesteros, I.; Ballesteros, M.; Cara, C.; Castro, E.; Ruiz, E. Different process configurations for bioethanol production from pretreated olive pruning biomass. J. Chem. Technol. Biotechnol. 2011, 86, 881-887. [CrossRef]

7. Negro, M.J.; Alvarez, C.; Ballesteros, I.; Romero, I.; Ballesteros, M.; Castro, E.; Manzanares, P.; Moya, M.; Oliva, J.M. Ethanol production from glucose and xylose obtained from steam exploded water-extracted olive tree pruning using phosphoric acid as catalyst. Bioresour. Technol. 2014, 153, 101-107. [CrossRef] [PubMed]

8. Toledano, A.; Alegría, I.; Labidi, J. Biorefining of olive tree (Olea europea) pruning. Biomass Bioenergy 2013, 59, 503-511. [CrossRef]

9. Solarte-Toro, J.C.; Romero-Garcia, J.M.; Lopez-Linares, J.C.; Ramos, E.R.; Castro, E.; Alzate, C.A.C. Simulation approach through the biorefinery concept of the antioxidants, lignin and ethanol production using olive leaves as raw material. Chem. Eng. Trans. 2018, 24, 1395-1406.

10. Castro, E.; Conde, E.; Moure, A.; Falqué, E.; Cara, C.; Ruiz, E.; Domínguez, H. Antioxidant activity of liquors from steam explosion of Olea europea wood. Wood Sci. Technol. 2008, 42, 579-592. [CrossRef]

11. Martínez-Patiño, J.C.; Gullón, B.; Romero, I.; Ruiz, E.; Brnčić, M.; Žlabur, J.Š.; Castro, E. Optimization of ultrasound-assisted extraction of biomass from olive trees using response surface methodology. Ultrason. Sonochem. 2019, 51, 487-495. [CrossRef]

12. Cara, C.; Ruiz, E.; Carvalheiro, F.; Moura, P.; Ballesteros, I.; Castro, E.; Gírio, F. Production, purification and characterisation of oligosaccharides from olive tree pruning autohydrolysis. Ind. Crops Prod. 2012, 40, 225-231. [CrossRef]

13. Toledano, A.; Serrano, L.; Labidi, J. Process for olive tree pruning lignin revalorisation. Chem. Eng. J. 2012, 193-194, 396-403. [CrossRef]

14. Krongtaew, C.; Messner, K.; Ters, T.; Fackler, K. Characterization of key parameters for biotechnological lignocellulose conversion assessed by FT-NIR spectroscopy. Part I: qualitative analysis of pretreated straw. BioResources 2010, 5, 2063-2080.

15. Gredilla, A.; Fdez-Ortiz de Vallejuelo, S.; Elejoste, N.; de Diego, A.; Madariaga, J.M. Non-destructive Spectroscopy combined with chemometrics as a tool for green chemical analysis of environmental samples: A review. Trends Anal. Chem. 2016, 76, 30-39. [CrossRef]

16. Skvaril, J.; Kyprianidis, K.G.; Dahlquist, E. Applications of near-infrared spectroscopy (NIRS) in biomass energy conversion processes: a review. Appl. Spectrosc. Rev. 2017, 52, 675-728. [CrossRef]

17. Xu, F.; Yu, J.; Tesso, T.; Dowell, F.; Wang, D. Qualitative and quantitative analysis of lignocellulosic biomass using infrared techniques: A mini-review. Appl. Energy 2013, 104, 801-809. [CrossRef]

18. Lupoi, J.S.; Singh, S.; Simmons, B.A.; Henry, R.J. Assessment of lignocellulosic biomass using analytical spectroscopy: an evolution to high-throughput techniques. BioEnergy Res. 2014, 7, 1-23. [CrossRef]

19. Sanderson, M.A.; Agblevor, F.; Collins, M.; Johnson, D.K. Compositional analysis of biomass feedstocks by near infrared reflectance spectroscopy. Biomass Bioenergy 1996, 11, 365-370. [CrossRef]

20. Payne, C.E.; Wolfrum, E.J. Rapid analysis of composition and reactivity in cellulosic biomass feedstocks with near-infrared spectroscopy. Biotechnol. Biofuels 2015, 8, 43. [CrossRef] [PubMed]

21. Liu, L.; Ye, X.P.; Womac, A.R.; Sokhansanj, S. Variability of biomass chemical composition and rapid analysis using FT-NIR techniques. Carbohydr. Polym 2010, 81, 820-829. [CrossRef]

22. Alves, A.; Santos, A.; Rozenberg, P.; Pâques, L.E.; Charpentier, J.-P.; Schwanninger, M.; Rodrigues, J. A common near infrared-based partial least squares regression model for the prediction of wood density of Pinus pinaster and Larix eurolepis. Wood Sci. Technol. 2012, 46, 157-175. [CrossRef] 
23. Nkansah, K.; Dawson-Andoh, B.; Slahor, J. Rapid characterization of biomass using near infrared spectroscopy coupled with multivariate data analysis: Part 1 yellow-poplar (Liriodendron tulipifera L.). Bioresour. Technol. 2010, 101, 4570-4576. [CrossRef] [PubMed]

24. Sandak, J.; Sandak, A. Fourier transform near infrared assessment of biomass composition of shrub willow clones (salix sp.) for optimal bio-conversion processing. J. Near Infrared Spec. 2011, 19, 309-318. [CrossRef]

25. Hayes, D.J.M. Development of near infrared spectroscopy models for the quantitative prediction of the lignocellulosic components of wet Miscanthus samples. Bioresour. Technol. 2012, 119, 393-405. [CrossRef] [PubMed]

26. Yang, Z.; Li, K.; Zhang, M.; Xin, D.; Zhang, J. Rapid determination of chemical composition and classification of bamboo fractions using visible-near infrared spectroscopy coupled with multivariate data analysis. Biotechnol. Biofuels 2016, 9, 35. [CrossRef] [PubMed]

27. Mata Sánchez, J.; Pérez Jiménez, J.A.; Díaz Villanueva, M.J.; Serrano, A.; Núñez, N.; López Giménez, J. Assessment of near infrared spectroscopy for energetic characterization of olive byproducts. Renew. Energy 2015, 74, 599-605. [CrossRef]

28. Sluiter, J.B.; Ruiz, R.O.; Scarlata, C.J.; Sluiter, A.D.; Templeton, D.W. Compositional analysis of lignocellulosic feedstocks. 1. review and description of methods. J. Agric. Food Chem. 2010, 58, 9043-9053. [CrossRef] [PubMed]

29. Savitzky, A.; Golay, M.J.E. Smoothing and differentiation of data by simplified least squares procedures. Anal. Chem. 1964, 36, 1627-1639. [CrossRef]

30. Kaiser, H.F. The application of electronic computers to factor analysis. Educ. Psychol. Meas. 1960, 20, 141-151. [CrossRef]

31. Haaland, D.M.; Thomas, E.V. Partial least-squares methods for spectral analyses. 2. Application to simulated and glass spectral data. Anal. Chem. 1988, 60, 1202-1208. [CrossRef]

32. Ballesteros, I.; Ballesteros, M.; Cara, C.; Sáez, F.; Castro, E.; Manzanares, P.; Negro, M.J.; Oliva, J.M. Effect of water extraction on sugars recovery from steam exploded olive tree pruning. Bioresour. Technol. 2011, 102, 6611-6616. [CrossRef] [PubMed]

33. Cara, C.; Ruiz, E.; Oliva, J.M.; Sáez, F.; Castro, E. Conversion of olive tree biomass into fermentable sugars by dilute acid pretreatment and enzymatic saccharification. Bioresour. Technol. 2008, 99, 1869-1876. [CrossRef] [PubMed]

34. Romero-García, J.M.; Lama-Muñoz, A.; Rodríguez-Gutiérrez, G.; Moya, M.; Ruiz, E.; Fernández-Bolaños, J.; Castro, E. Obtaining sugars and natural antioxidants from olive leaves by steam-explosion. Food Chem. 2016, 210, 457-465. [CrossRef] [PubMed]

35. Schwanninger, M.; Rodrigues, J.C.; Fackler, K. A review of band assignments in near infrared spectra of wood and wood components. J. Near Infrared Spec. 2011, 19, 287-308. [CrossRef]

36. Adjalle, K.; Larose, L.V.; Bley, J.; Barnabé, S. The effect of organic nitrogenous compound content and different pretreatments on agricultural lignocellulosic biomass characterization methods. Cellulose 2017, 24, 1395-1406. [CrossRef]

37. Poke, F.S.; Wright, J.K.; Raymond, C.A. Predicting extractives and lignin contents in Eucalyptus globulus using Near Infrared Reflectance Analysis. J. Wood Chem. Technol. 2005, 24, 55-67. [CrossRef]

38. Roy, K.; Kar, S.; Das, R.N. A Primer on QSAR/QSPR Modelling; Roy, K., Das, R.N., Eds.; Springer International Publishing: New York, NY, USA, 2015; pp. 37-39.

39. Veerasamy, R.; Rajak, H.; Jain, A.; Sivadasan, S.; Varghese, C.; Agrawal, R. Validation of QSAR models-Strategies and importance. Int. J. Drug Design Discovery 2011, 2, 511-519.

40. Kelly, S.S.; Rowell, R.M.; Davis, M.; Jurich, C.K.; Ibach, R. Rapid analysis of the chemical composition of agricultural fibers using near infrared spectroscopy and pyrolysis molecular beam mass spectroscopy. Biomass Bioenergy 2004, 27, 77-88. [CrossRef]

41. Chen, J.Y.; Matsunaga, R.; Ishikawa, K.; Zhang, H. Main inorganic component measurement of seawater using near-infrared spectroscopy. Appl. Spectrosc. 2003, 57, 1399-1406. [CrossRef]

(C) 2019 by the authors. Licensee MDPI, Basel, Switzerland. This article is an open access article distributed under the terms and conditions of the Creative Commons Attribution (CC BY) license (http://creativecommons.org/licenses/by/4.0/). 
Article

\title{
Ultrasound-Assisted Extraction as a First Step in a Biorefinery Strategy for Valorisation of Extracted Olive Pomace
}

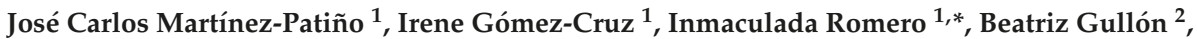 \\ Encarnación Ruiz ${ }^{1}$, Mladen Brnčić ${ }^{3}$ and Eulogio Castro ${ }^{1}$ \\ 1 Department of Chemical, Environmental and Materials Engineering, Universidad de Jaén, Campus Las \\ Lagunillas, 23071 Jaén, Spain \\ 2 Department of Chemical Engineering, Institute of Technology, Universidade de Santiago de Compostela, \\ 15782 Santiago de Compostela, Spain \\ 3 Department of Process Engineering, Faculty of Food Technology and Biotechnology, University of Zagreb, \\ Pierottijeva 6, HR-10000 Zagreb, Croatia \\ * Correspondence: iromero@ujaen.es; Tel.: +34-953213644
}

Received: 29 May 2019; Accepted: 10 July 2019; Published: 12 July 2019

\begin{abstract}
Currently, interest in finding new feedstock as sources of natural food antioxidants is growing. The extracted olive pomace (EOP), which is an agro-industrial residue from the olive pomace extracting industries, is generated yearly in big amounts, mainly in the Mediterranean countries. EOP was subjected to an ultrasound assisted extraction with ethanol-water mixtures. The effect of main parameters, such as ethanol concentration $(30-70 \% \mathrm{v} / \mathrm{v})$, ultrasound amplitude $(20-80 \%)$, and extraction time (5-15 $\mathrm{min}$ ), on the extraction of antioxidant compounds was evaluated according to a Box-Behnken experimental design. The antioxidant capacity of the resulting extracts was determined by measuring their content in total phenolic compounds (TPC) and flavonoids (TFC), as well as their antioxidant activity by DPPH, ferric reducing antioxidant power (FRAP), and ABTS assays. Considering the simultaneous maximization of these five responses, the optimal conditions were found to be $43.2 \%$ ethanol concentration, $70 \%$ amplitude, and $15 \mathrm{~min}$. The ultrasound assisted extraction of EOP under these optimized conditions yielded an extract with a phenolic and flavonoid content (per gram of EOP) of $57.5 \mathrm{mg}$ gallic acid equivalent (GAE) and $126.9 \mathrm{mg}$ rutin equivalent (RE), respectively. Likewise, the values for DPPH, ABTS, and FRAP assay (per gram of EOP) of 56.7, 139.1, and $64.9 \mathrm{mg}$ Trolox equivalent, respectively were determined in the optimized extract.
\end{abstract}

Keywords: extracted olive pomace; response surface methodology; phenolic compounds; flavonoids; antioxidant activity

\section{Introduction}

In recent years, the demand for natural antioxidants as alternatives to synthetic antioxidants has increased. Phenolic compounds, such as flavonoids, phenolic acids, phenolic alcohols, stilbenes, lignans, and tannins, exhibit antioxidant activities and other types of bioactivity (anti-inflammatory, anticarcinogenic, antiviral, hepatoprotective, and neuroprotective activities, among others) [1,2]. These properties make these compounds useful in the food and pharmaceutical industries as food preservatives and biocompounds for human health [3]. The residual biomass from agro-industrial processes could be a cheap and abundant source of high-added value compounds. The valorisation of these by-products within the context of biorefineries that produce biofuels, power, and chemicals within a single installation might help in diversifying the economies of rural areas and developing an industrial complex [4]. 
Olive oil consumption has become a worldwide phenomenon due to its healthy properties. Olive oil production has important social and economic effects in Mediterranean countries. Spain leads the list of olive oil-producing countries and generated more than six million tons of olives in 2017 [5]. Several by-products and residues are generated during the olive oil production process and they require appropriate disposal. Olive pomace is the major by-product in olive oil mills and it represents nearly $70-80 \%$ of the weight of olives [6,7]. Currently, most of this agro-industrial by-product is dried and extracted with an organic solvent, such as hexane, to recover the residual oil that remains in the olive pomace, which represents $1-3 \%$ of its total weight [6]. The final solid waste that was produced by olive pomace-extracting industries is called extracted olive pomace (EOP). This biomass represents around $20 \%$ of the pomace dry weight, and thus more than one million tons of this waste is produced yearly in Spain. The olive skins, pulp, seeds, and different proportions of pieces of stone form this exhausted solid with a moisture level of approximately $10 \%$ [8]. Currently, the stone content in EOP is diminishing, because stone fragments are generally recovered from pomace for use as fuel, mainly in domestic heating systems. With respect to the current use of EOP, the main part of this residue is burnt as a biofuel in small factories or in electric plants. However, different environmental problems that occur during EOP combustion, such as particle emission and hazardous gas generation, hinder this valorisation option [7]. Several phenolic compounds with antioxidant activities have been detected in residual biomass from the olive oil production process, which include hydroxytyrosol, tyrosol, oleuropein, ligustroside, apigenin, luteolin, rutin, vanillic acid, and caffeic acid $[9,10]$. Therefore, this residue is an interesting source of high-value bioactive compounds. Additionally, the removal of part of the non-structural fraction could improve the further energetic use of the biomass [7].

New extraction technologies have been used to overcome some of the disadvantages of the classical extraction techniques, such as maceration or the use of a Soxhlet extractor. Among these techniques, ultrasound-assisted extraction (UAE) reduces the extraction time and the solvent and energy consumption [11]. Additionally, this method achieves higher extraction yields and it is easy to scale up. UAE induces the formation of bubbles (which growth and collapse) in the irradiated liquid causing cavitation [12]. These phenomena cause mechanic, thermal, and chemical effects inside the samples. Thus, a higher extraction rate is achieved due to the structural changes in the solid sample that enhance mass transport. Moreover, the increase in temperature improves the diffusion and the solubility of the polyphenols [13]. Bath and probe ultrasound devices are the principal types of ultrasound equipment. In an ultrasonic probe system, the energy is applied directly to the sample, the energy losses are therefore minimised, and the extraction yields are increased [14]. Ultrasonic power, frequency, amplitude, extraction time, temperature, liquid-solid ratio, solvent composition, and the matrix properties are the main parameters in the UAE process [15]. The use of a mathematical tool, such as response surface methodology (RSM), can be useful in optimising and studying the effects of different operational parameters of UAE on the responses. RSM has been previously applied to study the UAE of antioxidants from different agro-industrial biomasses [11,13].

In the present work, EOP was subjected to UAE as a first step in a global strategy to valorise olive-derived biomass to produce energy and chemicals via the biorefinery approach. The aim of this work was evaluate the influence of three operational parameters (i.e., water/ethanol ratio, ultrasound amplitude, and extraction time) and to determine their optimal values while using RSM to maximise the flavonoid and phenolic compound contents and the antioxidant activities of the EOP extracts, which were determined by various tests (DPPH, ABTS, and ferric reducing antioxidant power (FRAP)). Additional experiments under the conditions that were optimized by simultaneously considering the five analysed responses were performed to evaluate the adequacy of the model equations. To our best knowledge, this is the first time that real industrial exhausted olive pomace is ultrasound-assisted extracted. 


\section{Materials and Methods}

\subsection{Raw Material}

EOP was collected during the olive oil season from the olive pomace extracting industry functionary "Oleocastellar S.A.", which is located in the province of Jaén. The EOP was homogeneized and air-dried until moisture equilibrium was achieved. Afterwards, the EOP was milled with a $1 \mathrm{~mm}$ sieve and then stored in a dry place until use.

\subsection{Ultrasound-Assisted Extraction (UAE) and Experimental Design}

The extraction of the EOP was studied according to the Box-Behnken design, a type of response surface methodology with three levels of each factor, which allows for reducing the number of experiments that are needed to provide enough information for statistically acceptable results. In the Box-Behnken design, each variable or factor adopts three levels and the experiments are distributed at the midpoints of the edges and at the centre of the cube that was formed by the factor levels. It constitutes a typical experimental design when fitting the quadratic models.

The extraction of EOP was carried out in an ultrasound probe device (UP400S, Hielscher, Germany) with a $22 \mathrm{~mm}$ diameter sonotrode. The maximum power was $400 \mathrm{~W}$ and the ultrasonic frequency was $24 \mathrm{kHz}$. The ethanol concentration (20,50, 80\%), ultrasound amplitude $(30,50,70 \%)$, and extraction time $(5,10,15 \mathrm{~min})$ were the three variables studied (Table 1). Table 1 presents the natural and coded values of these factors. The biomass was milled and sieved while using a cutting mill (Ultra Centrifugal Mill Retsch ZM 200, Haan, Germany) to $1 \mathrm{~mm}$ particle size, and the liquid-solid ratio was $20 \mathrm{~mL} / \mathrm{g}$ (i.e., $15 \mathrm{~g}$ of EOP and $300 \mathrm{~mL}$ of solvent inside a $400 \mathrm{~mL}$ beaker). The sonotrode was submerged by $1.5 \mathrm{~cm}$ in the samples. The samples were not cooled and therefore the temperatures of the samples increased during the extraction. Once the extraction was completed, the samples were vacuum filtered and the extracts were stored at $-18^{\circ} \mathrm{C}$.

Table 1. Uncoded and coded values of the factors.

\begin{tabular}{cccccc}
\hline \multirow{2}{*}{ Independent Variable } & \multirow{2}{*}{ Nomenclature } & \multirow{2}{*}{ Units } & \multicolumn{3}{c}{ Value } \\
& & & $\mathbf{( - 1 )}$ & $\mathbf{0}$ & $\mathbf{( + 1 )}$ \\
\hline Ethanol concentration & $\mathrm{EtOH}$ & $\% \mathrm{v} / \mathrm{v}$ & 20 & 50 & 80 \\
Amplitude & $\mathrm{Amp}$ & $\%$ & 30 & 50 & 70 \\
Extraction time & $\mathrm{t}$ & $\mathrm{min}$ & 5 & 10 & 15 \\
\hline
\end{tabular}

The influence of each variable was determined according to a second-order polynomial equation, which relates the independent variables $\left(x_{i}\right.$ and $\left.x_{j}\right)$ in the coded values with the response $\left(y_{i}\right)$ through the regression coefficients $\left(\beta_{0}, \beta_{i}, \beta_{i j}\right.$, and $\left.\beta_{i i}\right)$, as shown in Equation (1).

$$
y_{j}=\beta_{0}+\sum \beta_{i} \cdot x_{i}+\sum \beta_{i i} \cdot x_{i}^{2}+\sum \sum \beta_{i j} \cdot x_{i} \cdot x_{j}
$$

The experimental data were analysed with commercial software (Design Expert 7.0.0, Stat-Ease Inc., Minneapolis, MN, USA). The model predicted the optimal UAE conditions that were determined and experimentally tested in triplicate to evaluate its adequacy.

\subsection{Antioxidant Capacity Indicators for EOP}

The total phenolic compounds were measured by spectrophotometry, following the Folin-Ciocalteu method [16], with some modifications. Briefly, $0.5 \mathrm{~mL}$ of diluted extract was added to $3.875 \mathrm{~mL}$ of water and $0.125 \mathrm{~mL}$ of Folin-Ciocalteu reagent. Subsequently, $0.5 \mathrm{~mL}$ of a solution of $\mathrm{Na}_{2} \mathrm{CO}_{3}(10 \% \mathrm{w} / \mathrm{v})$ was added and the mixture was homogenised. After $1 \mathrm{~h}$, the absorbance was measured at $765 \mathrm{~nm}$ [17]. Gallic acid was used as a reference standard compound and the results are expressed as mg of gallic acid equivalent (GAE)/g EOP. 
The flavonoid content was measured according to a colorimetric method that Kim et al., describe [18]. In short, $1 \mathrm{~mL}$ of diluted extract was added to $0.3 \mathrm{~mL}$ of a solution of $\mathrm{NaNO}_{2}(5 \% \mathrm{w} / \mathrm{v})$, followed by $0.3 \mathrm{~mL}$ of $10 \% \mathrm{AlCl}_{3}$ solution after $5 \mathrm{~min}$, and the resultant solution was homogenised. Six minutes later, $2 \mathrm{~mL}$ of $\mathrm{NaOH}$ solution $1 \mathrm{M}$ was added, and the resultant solution was mixed. After $5 \mathrm{~min}$, the absorbance was measured at $510 \mathrm{~nm}$. Rutin was used as the reference standard, and the results are expressed as $\mathrm{mg}$ of rutin equivalents $(\mathrm{RE}) / \mathrm{g}$ EOP. All of the measurements were performed in triplicate and the mean values are reported.

Three different assays widely known, DPPH, ABTS, and FRAP, were performed to know the antioxidant activity of the EOP extracts. In the DPPH radical scavenging assay, $2 \mathrm{~mL}$ of $6 \times 10^{-5} \mathrm{M}$ methanolic solution of DPPH (2,2-diphenyl-1-picrylhydrazyl) was added to a $200 \mu \mathrm{L}$ of sample. The reduction of absorbance was measured at $517 \mathrm{~nm}$ after $15 \mathrm{~min}$.

The TEAC (Trolox equivalent antioxidant capacity) method is based on the scavenging of ABTS radical (2,2'-azino-di(3-ethyl-benzothiazoline-6-sulfonic acid)). ABTS radical cation (ABTS·+) was originated by reacting $7 \mathrm{mM}$ ABTS stock solution with $2.45 \mathrm{mM}$ potassium persulfate, which allowed the mixture to stand at room temperature and protected it from the light for 12-16 h before use. Afterwards, ABTS- + solution was diluted with phosphate buffer (PBS, pH7.4) to an absorbance of 0.7 at $734 \mathrm{~nm}$. The decrease in absorbance was read at $6 \mathrm{~min}$ after the addition of $2.0 \mathrm{~mL}$ of diluted ABTS·+ solution to $20 \mu \mathrm{L}$ of samples.

The FRAP (ferric reducing antioxidant power) reagent was prepared with $200 \mathrm{mmol} / \mathrm{L}$ acetate buffer (pH 3.6), $10 \mathrm{mmol} / \mathrm{L}$ of 2,4,6-tripyridyl-s-triazine (TPTZ) solution in $40 \mathrm{mmol} / \mathrm{L} \mathrm{HCl}$, and $20 \mathrm{mmol} / \mathrm{L}$ $\mathrm{FeCl} 3.6 \mathrm{H} 2 \mathrm{O}$ in distilled water in the proportion of 10:1:1, respectively. The samples $(100 \mu \mathrm{L})$ were mixed with the FRAP reagent $(3 \mathrm{~mL})$ and the absorbance was measured at $593 \mathrm{~nm}$ after $6 \mathrm{~min}$. Trolox was used as the standard and the antioxidant activities are expressed as mg of trolox equivalent (TE)/g EOP. All of the samples were analysed in triplicate.

\section{Results and Discussion}

\subsection{Influence of the Factors on the UAE of EOP and Model Equations}

The Box-Behnken design was applied to study the impact of the three variables (ethanol concentration, amplitude, and ultrasonic time) on the ultrasound-assisted extraction of EOP. A total of 17 experiments, including one point and four replicates at the centre of the domain selected for each factor, were performed in random order (Table 2). Ethanol-water mixtures were selected as the extraction solvents following results that were obtained in our laboratory with other olive residues [17]. Additionally, ethanol is a low-cost and non-toxic solvent that is used in several food applications. Regarding the time and amplitude, the experimental range was not extended to avoid solvent evaporation, as the samples were not refrigerated during the experiment. The final temperature exceeded $75{ }^{\circ} \mathrm{C}$ (experiments 3 and 16) in the cases with higher values for both parameters. Table 2 presents the final temperatures of all of the experiments. The values of the fixed variables, such as particle size and the solid-liquid ratio, were selected based on the results of a preliminary screening [17].

Table 3 summarises the different statistical parameters and demonstrates the model adjustment to the experimental data for each model equation. The models that were obtained for each of the responses were statistically significant, with p-values $<0.0001$ in all cases, and the dispersions of the experimental results were independent of the pure errors of the models, as indicated by the lack of significance of the $p$-values for the lack of fit ( $p>0.05$ in all cases). $R^{2}$ values that are close to 1 indicate good accuracies of the models and, in all cases, the $\mathrm{R}^{2}$ values were greater than 0.97 . Moreover, the good precision and reproducibility of the models were confirmed by the CV values, which were below $3.5 \%$ in the five response equations. 
Table 2. Experimental conditions for ultrasound-assisted extraction (UAE) for extracted olive pomace (EOP) and experimental data for the responses.

\begin{tabular}{cccccccccc}
\hline Exp. & $\begin{array}{c}\text { EtOH } \\
(\mathbf{\% v} / \mathbf{v})\end{array}$ & $\begin{array}{c}\text { Amp } \\
\mathbf{( \% )}\end{array}$ & $\begin{array}{c}\text { Time } \\
(\mathbf{m i n})\end{array}$ & $\begin{array}{c}\text { T } \\
\left({ }^{\circ} \mathbf{C}\right)\end{array}$ & $\begin{array}{c}\text { TPC }(\mathbf{m g} \\
\text { GAE/g EOP) }\end{array}$ & $\begin{array}{c}\text { TFC }(\mathbf{m g} \\
\text { RE/g EOP) }\end{array}$ & $\begin{array}{c}\text { DPPH (mg } \\
\text { TE/g EOP) }\end{array}$ & $\begin{array}{c}\text { ABTS (mg } \\
\text { TE/g EOP) }\end{array}$ & $\begin{array}{c}\text { FRAP (mg } \\
\text { TE/g EOP) }\end{array}$ \\
\hline 1 & 50 & 50 & 10 & 59 & 55.8 & 119.7 & 53.6 & 126.0 & 58.9 \\
2 & 20 & 30 & 10 & 47 & 53.0 & 113.9 & 50.7 & 121.6 & 56.9 \\
3 & 50 & 70 & 15 & 76 & 60.4 & 125.6 & 59.9 & 140.5 & 64.4 \\
4 & 50 & 50 & 10 & 58 & 54.5 & 118.2 & 56.6 & 128.0 & 61.0 \\
5 & 20 & 50 & 5 & 44 & 51.1 & 105.2 & 51.8 & 103.6 & 51.6 \\
6 & 50 & 50 & 10 & 58 & 54.2 & 117.4 & 55.8 & 124.3 & 58.3 \\
7 & 50 & 30 & 5 & 41 & 54.1 & 113.2 & 52.3 & 116.7 & 51.8 \\
8 & 50 & 30 & 15 & 57 & 53.3 & 116.0 & 58.3 & 128.2 & 57.5 \\
9 & 50 & 70 & 5 & 54 & 54.7 & 114.5 & 59.3 & 122.9 & 57.7 \\
10 & 50 & 50 & 10 & 59 & 54.3 & 119.4 & 56.1 & 125.2 & 57.5 \\
11 & 80 & 50 & 15 & 64 & 37.3 & 91.2 & 45.3 & 84.8 & 43.2 \\
12 & 80 & 70 & 10 & 69 & 41.3 & 88.0 & 44.0 & 90.4 & 44.2 \\
13 & 80 & 50 & 5 & 44 & 35.7 & 81.1 & 40.5 & 72.1 & 36.6 \\
14 & 80 & 30 & 10 & 44 & 32.8 & 83.1 & 40.8 & 74.6 & 36.1 \\
15 & 50 & 50 & 10 & 59 & 52.5 & 118.4 & 56.1 & 128.3 & 58.8 \\
16 & 20 & 70 & 10 & 74 & 53.5 & 110.0 & 52.7 & 119.3 & 55.6 \\
17 & 20 & 50 & 15 & 64 & 51.3 & 113.2 & 52.1 & 116.9 & 52.8 \\
\hline
\end{tabular}

GAE: gallic acid equivalent; RE: rutin equivalent; TE: trolox equivalent.

Table 3. Statistical parameters of the five model equations.

\begin{tabular}{cccccc}
\hline Coefficient & TPC & TFC & DPPH & ABTS & FRAP \\
\hline F-value & 147.14 & 166.67 & 76.85 & 192.12 & 60.44 \\
p-value & $<0.0001$ & $<0.0001$ & $<0.0001$ & $<0.0001$ & $<0.0001$ \\
$\mathrm{R}^{2}$ & 0.9913 & 0.9901 & 0.9835 & 0.9934 & 0.9734 \\
Adj R & 0.9843 & 0.9842 & 0.9707 & 0.9882 & 0.9571 \\
C.V & 1.98 & 1.61 & 2.00 & 1.96 & 3.24 \\
Lack of fit (p-value) & 0.8047 & 0.0706 & 0.6751 & 0.2425 & 0.2274 \\
\hline
\end{tabular}

The experimental results were adjusted to quadratic regression equations. The equations for the coded values of the independent variables that modelled the five studied responses are presented below, with the non-significant terms ( $\mathrm{p}$-values $>0.1$ ) removed.

$$
\begin{gathered}
\mathrm{TPC}=54.28-7.73 \cdot \mathrm{EtOH}+2.08 \cdot \mathrm{Amp}+0.84 \cdot \mathrm{t}+1.99 \cdot \mathrm{EtOH} \cdot \mathrm{Amp}+1.63 \cdot \mathrm{Amp} \cdot \mathrm{t}-10.44 \mathrm{EtOH}^{2}+1.32 \cdot \mathrm{Amp}^{2} \\
\mathrm{TFC}=118.05-12.35 \cdot \mathrm{EtOH}+1.5 \cdot \mathrm{Amp}+4 \cdot \mathrm{t}+2.19 \cdot \mathrm{EtOH} \cdot \mathrm{Amp}+2.08 \cdot \mathrm{Amp} \cdot \mathrm{t}-19.82 \mathrm{EtOH}^{2} \\
\mathrm{DPPH}=55.94-4.60 \cdot \mathrm{EtOH}+1.73 \cdot \mathrm{Amp}+1.45 \cdot \mathrm{t}+1.12 \cdot \mathrm{EtOH} \cdot \mathrm{t}-1.34 \cdot \mathrm{Amp} \cdot \mathrm{t}-9.27 \mathrm{EtOH}^{2}+1.16 \cdot \mathrm{t}^{2} \\
\mathrm{ABTS}=126.37-17.46 \cdot \mathrm{EtOH}+4.02 \cdot \mathrm{Amp}+6.88 \cdot \mathrm{t}+4.54 \cdot \mathrm{EtOH} \cdot \mathrm{Amp}-28.78 \mathrm{EtOH}^{2}+3.91 \cdot \mathrm{Amp}^{2}-3.22 \cdot \mathrm{t}^{2} \\
\quad \mathrm{FRAP}=59.13-7.11 \cdot \mathrm{EtOH}+2.44 \cdot \mathrm{Amp}+2.52 \cdot \mathrm{t}+2.35 \cdot \mathrm{EtOH} \cdot \mathrm{Amp}-11.2 \mathrm{EtOH}^{2}+1.57 \cdot \mathrm{t}^{2}
\end{gathered}
$$

\subsubsection{Total Phenolic Compounds (TPC)}

Phenolic compounds are metabolites with antioxidant activities that are present in vegetable sources. These compounds are highly sensitive to environmental factors; therefore, the extraction conditions are very important in recovering high levels of bioactive compounds [1]. As presented in Table 2, the amounts of TPC ranged between 32.8 and $60.4 \mathrm{mg}$ GAE/g EOP in the experiments numbered 14 and 3 of the design. The model equation (Equation (2)) revealed that the ethanol concentration was the most influential factor in this response, as can be deduced from the higher coefficients of the linear and quadratic terms for this variable. Likewise, the influence of the other two factors, ultrasound amplitude and extraction time, as well as the interaction between them, was significant. 
As an example, Figure 1 shows a close agreement between the predicted and experimental values for TPC.

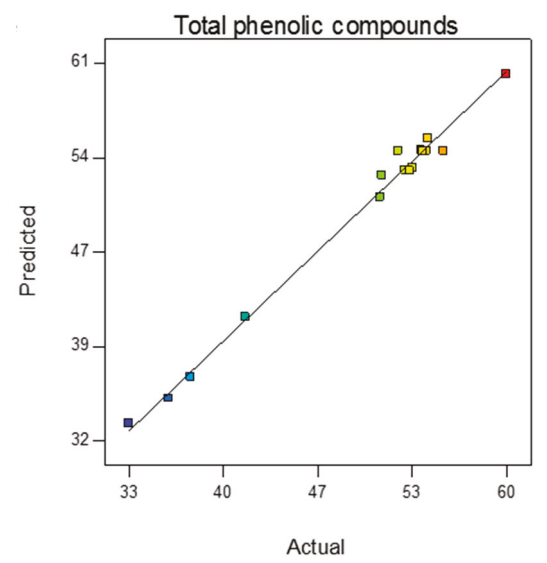

Figure 1. Predicted versus experimental values for total phenolic compounds (TPC) content in the EOP extracts.

As presented in Figure 2a, the ethanol concentration had a positive influence that reached a maximum at approximately $40 \%$ ethanol and subsequently declined, as indicated by the negative signs of the linear and quadratic terms for this parameter. This behaviour can be explained in accordance with the principle of similarity and intermiscibility; i.e., greater extraction of phenolic compounds is achieved when the solvent and the solute have similar polarity [2]. Notably, the use of a mixture of ethanol and water, as the solvent is more efficient in the phenolic extraction than the use of a pure solvent. Goldsmith et al. [19] optimised the UAE of olive pomace while only using water and achieved a maximum of $19.7 \mathrm{mg} \mathrm{GAE} / \mathrm{g}$ OP, which is lower than the values that were obtained in the present work. The amplitude and extraction time had a positive effect over the entire range (Figure $2 \mathrm{~b}$ ) and amplitude had the greater positive effect. Thus, the highest TPC (60.9 mg GAE/g EOP) was predicted by the model at an ethanol concentration of $41.7 \%$, an amplitude of $70 \%$, and a time of $15 \mathrm{~min}$.

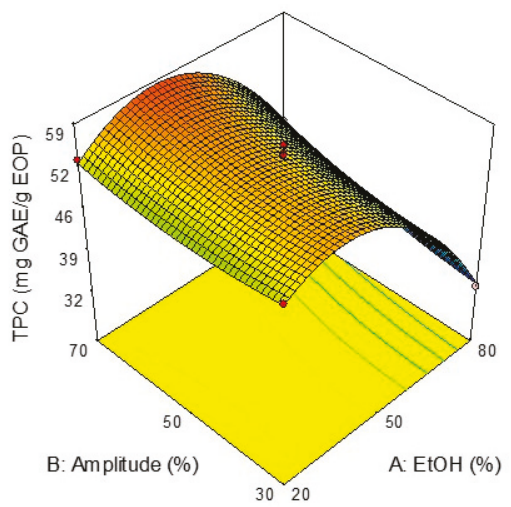

(a)

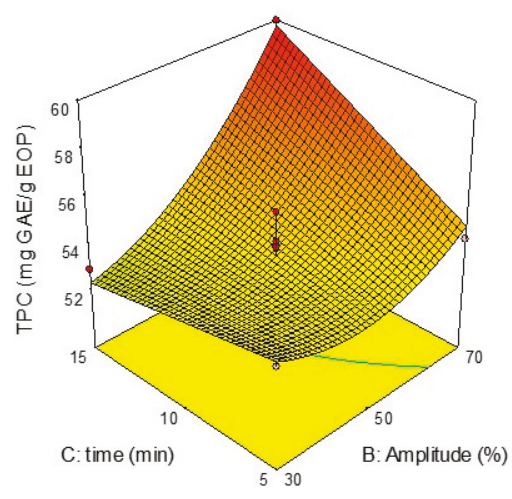

(b)

Figure 2. Response surfaces of the total phenolic compounds (TFC) as functions of the (a) ethanol concentration and amplitude (time: $10 \mathrm{~min}$ ) and the $(\mathbf{b})$ amplitude and extraction time (ethanol concentration: $50 \%)$. 


\subsubsection{Total Flavonoid Compounds (TFC)}

Flavonoids are a subgroup within the highly diverse group of phenolic compounds, and their specific biological properties depend on their chemical structures. The TFC values ranged between 81.1 (experiment 13) and $125.6 \mathrm{mg} \mathrm{RE/g} \mathrm{EOP} \mathrm{(experiment} \mathrm{3)} \mathrm{in} \mathrm{the} \mathrm{performed} \mathrm{experiments} \mathrm{(Table} \mathrm{2).} \mathrm{These}$ results indicate that the extraction yield of flavonoids increased up to 1.55 times when the extraction was carried out at the intermediate level of ethanol concentration and the highest level of both ultrasound amplitude and time. This shows the importance of optimising the UAE conditions. As expected, taking into account that flavonoids can be considered as phenolic compounds, the predicted model for TFC (Equation (3)) was similar to that obtained for TPC with a significant negative effect of the ethanol concentration in both responses.

Figure 2 depicts the surface of the TPC response as a function of ethanol concentration and amplitude (a) and as a function of amplitude and time (b). The extraction time and amplitude exhibited positive influences over the entire studied range. The TFC level reached a maximum at a solvent composition of approximately $40 \%$ ethanol regarding the influence of the ethanol concentration (Figure 3a), and again this was the most important factor. Similar influence of the ethanol concentration in flavonoid extraction has been observed in other biomasses [2,18]. In this work, the model predicted the greatest TFC at $42.3 \%$ ethanol concentration, 70\% amplitude, and $15 \mathrm{~min}$, which would achieve $126.9 \mathrm{mg}$ RE/g EOP.

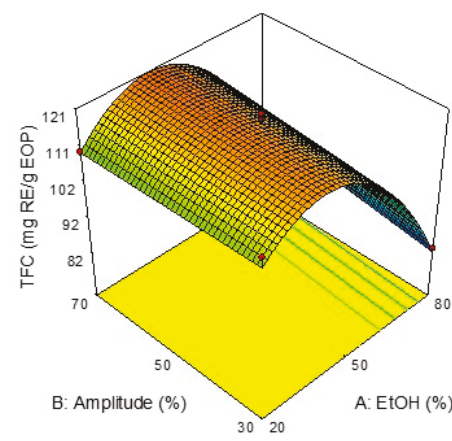

(a)

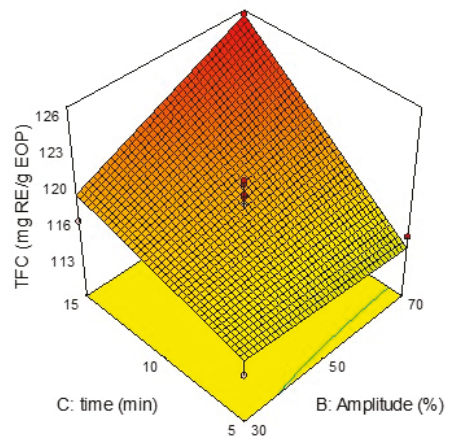

(b)

Figure 3. Response surface plots of the total flavonoid content (TFC) as a function of (a) the ethanol concentration and amplitude (time: $10 \mathrm{~min}$ ) and (b) the amplitude and time (ethanol concentration: 50\%).

\subsubsection{Antioxidant Activity}

Three methods were applied to determine the antioxidant activities in the EOP extracts. The experimental values (Table 2) varied between 40.5 and $59.9 \mathrm{mg}$ TE/g EOP in the DPPH assay, between 72.1 and $140.5 \mathrm{mg}$ TE/g EOP in the ABTS assay, and between 36.1 and $64.4 \mathrm{mg} \mathrm{TE} / \mathrm{g}$ EOP in the FRAP assay. The software generated similar model equations for DPPH Equation (4), ABTS Equation (5), and FRAP assays Equation (6).

In the three analytical methods that were applied, the antioxidant activity depended on the three variables studied, with the linear and quadratic terms for the ethanol concentration being the most significant ones with a clear negative influence. It is worth noting that a significant interaction effect between this factor and the ultrasound amplitude was detected for ABTS and FRAP assays, but not for DPPH. In the case of ABTS assay, the negative coefficient for the quadratic term for extraction time was not sufficient to change the trend. Figure $4 \mathrm{a}, \mathrm{c}$,e depict the influence of ethanol concentration and amplitude on the DPPH, ABTS, and FRAP assay responses, respectively. Figure $4 \mathrm{~b}, \mathrm{~d}, \mathrm{f}$ present the surface responses as a function of amplitude and extraction time for the same responses. The three assays of antioxidant activity produced similar influences of the studied variables on the TPC and TFC 
responses. This behaviour indicates a clear correlation between the presence of phenolic compounds and the antioxidant activity of the EOP extracts. The extraction time and amplitude increased with the values of the responses across the entire studied ranges, and the ethanol concentration produced maximum yield when the liquid was approximately $40 \%$ ethanol.

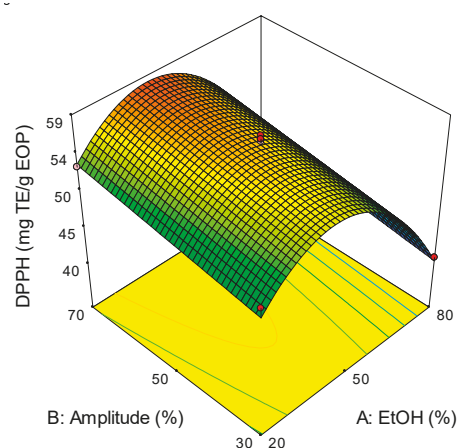

(a)

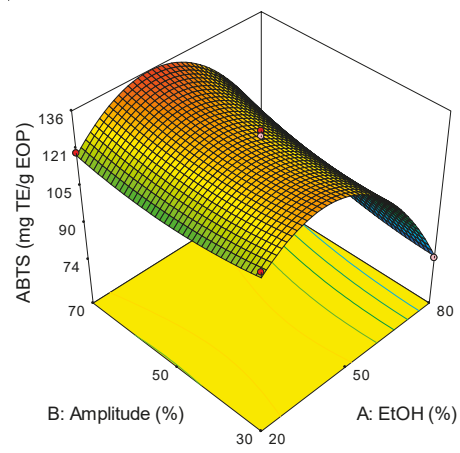

(c)

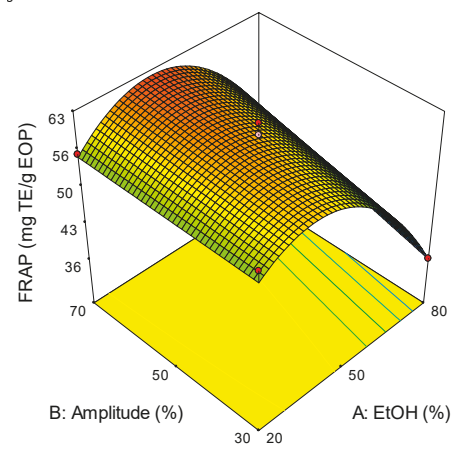

(e)

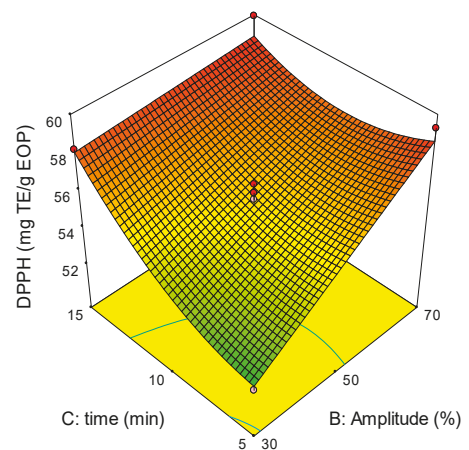

(b)

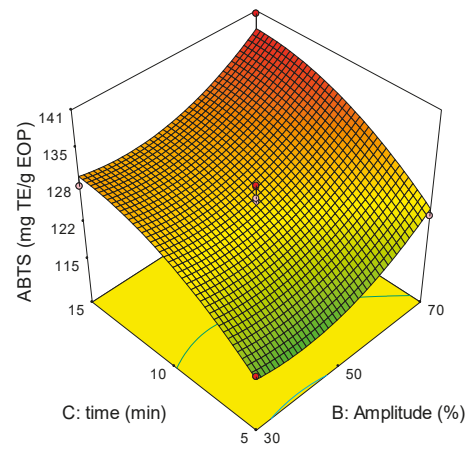

(d)

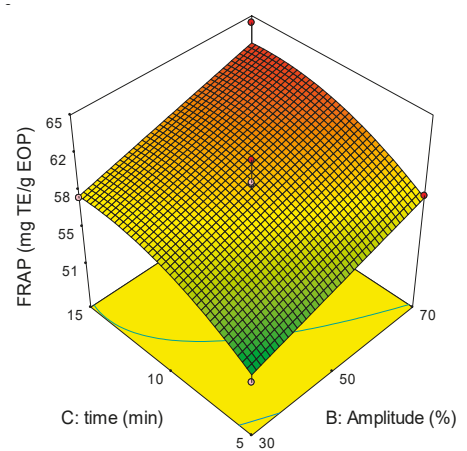

(f)

Figure 4. Response surfaces of the antioxidant activity of the extracted olive pomace (EOP) extracts. (a,b) DPPH assay; (c,d) ABTS assay; and, (e,f) FRAP assay. 


\subsection{Process Optimisation and Validation of the Model}

An optimisation of the three studied variables was carried out with the objective of simultaneously maximising the five measured responses due to the relationship between phenolic compounds and their antioxidant activity (i.e., TPC, TFC, DPPH, ABTS, and FRAP). The optimal conditions that were predicted by the model that maximised all responses included a $43.2 \%$ ethanol concentration, $70 \%$ amplitude, and a $15 \mathrm{~min}$ extraction time. Table 4 shows the values that were predicted by the model for all of the responses in the optimal conditions and the means of three experiments that were performed in the optimal conditions to test the adequacy of the model. The experimental data were close to the values that were predicted by the model; the error was below $10 \%$ in all cases.

Table 4. Predicted and experimental ultrasound-assisted etraction (UAE) values of EOP in the optimal conditions that simultaneously maximised the five responses.

\begin{tabular}{cccccc}
\hline Values & TPC $(\mathbf{m g}$ & TFC $(\mathbf{m g}$ & DPPH $(\mathbf{m g}$ & ABTS $(\mathbf{m g}$ & FRAP $(\mathbf{m g}$ \\
& GAE/g EOP) & RE/g EOP) & TE/g EOP) & TE/g EOP) & TE/g EOP) \\
\hline Predicted value & 60.9 & 126.9 & 59.3 & 139.4 & 63.0 \\
Experimental value & $57.5 \pm 0.3$ & $139.6 \pm 2.3$ & $56.7 \pm 1.5$ & $139.1 \pm 4.7$ & $64.9 \pm 0.6$ \\
Error & $5.9 \%$ & $9.1 \%$ & $4.6 \%$ & $0.2 \%$ & $2.9 \%$ \\
\hline
\end{tabular}

Regarding other residues from olive oil production, Martinez-Patiño et al. [17] studied the phenolic content and antioxidant activity of extracts from olive tree pruning (OTP) and olive mill leaves (OML) while also using UAE. The optimised UAE conditions for OTP and OML were similar to those for EOP, but the ethanol concentration was slightly higher (approximately 55\%). All of the responses that were determined for the EOP extracts were significantly greater than those of the OTP and OML extracts. Table 5 summarises the increments in the five studied responses in the EOP extracts with respect to OTP and OML. In the case of TPC, the EOP liquids produced results that were 1.85 times greater than those from the OTP extracts and 1.37 times greater than those of the OML extracts.

Table 5. Comparison of the main antioxidant activity indicators for EOP and other olive oil production residues.

\begin{tabular}{cccccc}
\hline Residue Ratio & TPC & TFC & DPPH & ABTS & FRAP \\
\hline EOP/OTP & 1.85 & 1.88 & 1.79 & 2.09 & 1.78 \\
EOP/OML & 1.37 & 1.45 & 1.33 & 1.45 & 1.31 \\
\hline
\end{tabular}

Other by-products from agro-industrial processes have been studied in terms of obtaining phenolic compounds while also using UAE. For example, waste from sunflower oil production, e.g., sunflower seed cake, reached $17.96 \mathrm{mg}$ GAE/g dry TPC biomass in optimised UAE conditions, $43 \%$ ethanol, $70{ }^{\circ} \mathrm{C}$ and $86 \mu \mathrm{m}$ amplitude [20], and the residue from lemon juice production produced $17.97 \mathrm{mg} \mathrm{GAE} / \mathrm{g}$ dry TPC biomass and an antioxidant activity of $9.4 \mathrm{mg} \mathrm{TE} / \mathrm{g}$ dry biomass in a FRAP assay of extracts that were obtained with UAE at $50{ }^{\circ} \mathrm{C}, 45 \mathrm{~min}$ and $250 \mathrm{~W}$ [21]. In both cases, the reported phenolic compound concentrations are lower than those that were obtained with EOP extracts in this work. Hence, EOP could be considered to be a relevant source for antioxidant compounds, in an olive-derived biorefinery concept.

\subsection{Olive Pomace and Extracted Olive Pomace as Sources of Antioxidants}

Olive pomace (OP) and extracted olive pomace (EOP), respectively, are the main wastes in the olive mills and the olive pomace extracting industries. Table 6 presents recent references from the literature related to the extraction of phenolic compounds from these agro-industrial residues. OP is the by-product obtained after olive oil separation (by centrifugation), which still contains residual oil and it is described with different names that include alperujo or olive cake while orujillo is the 
final residue, exhausted, and dry olive pomace. Most of these references used olive pomace from olive mills as raw material. In most of these works, olive pomace from the olive mills was used as raw material, and then it was defatted in the laboratory with an organic solvent prior to antioxidant extraction. Only the present work and that of Caballero et al. [22] used real EOP industrial waste.

Table 6. Antioxidant capacity indicators for olive pomace extracts.

\begin{tabular}{|c|c|c|c|c|}
\hline Material & Method & Conditions & Characterization of the Extracts & Ref. \\
\hline Olive pomace & $\begin{array}{l}\text { Ultrasound assisted } \\
\text { extraction }\end{array}$ & Water, $30^{\circ} \mathrm{C}, 75 \mathrm{~min}$ & $\begin{array}{c}\text { TPC: } 19.7 \text { mg GAE/g } \\
\text { DPPH: } 31.2 \mathrm{mg} \mathrm{TE} / \mathrm{g} ; \\
\text { CUPRAC: } 73.5 \mathrm{mg} \text { TE/g }\end{array}$ & [19] \\
\hline $\begin{array}{l}\text { Orujillo } \\
\text { (exhausted } \\
\text { pomace) }\end{array}$ & $\begin{array}{l}\text { Supercritical fluid } \\
\text { extraction }\end{array}$ & $\begin{array}{l}\text { Ethanol }(60 \%) \text {, pressure } 200,250 \text { and } \\
\text { 300 bar }\end{array}$ & $\begin{array}{l}\text { TPC: } 14.0 \mathrm{mg} \mathrm{GAE} / \mathrm{g} \\
\text { DPPH: } 85.3 \mu \mathrm{g} \mathrm{TE} / \mathrm{mL}\end{array}$ & [22] \\
\hline \multirow{5}{*}{ Olive pomace } & \multirow{5}{*}{$\begin{array}{l}\text { Homogenate (HAE), } \\
\text { microwave (MAE), } \\
\text { ultrasound (UAE) and } \\
\text { high hydrostatic } \\
\text { pressure (HHPAE) } \\
\text { assisted extraction }\end{array}$} & Natural deep eutectic solvents: & & \multirow{5}{*}{ [23] } \\
\hline & & $\operatorname{HAE}\left(60^{\circ} \mathrm{C}, 30 \mathrm{~min}, 12,000 \mathrm{rpm}\right)$ & $\begin{array}{c}\text { TPC: } 34 \text { mg GAE } / g ; 28 \text { g d.w/g } \\
\text { DPPH }\end{array}$ & \\
\hline & & $\operatorname{MAE}\left(60^{\circ} \mathrm{C}, 30 \mathrm{~min}, 200 \mathrm{~W}\right)$ & $\begin{array}{c}\text { TPC: } 29.6 \mathrm{mg} \mathrm{GAE} / \mathrm{g} ; 36.7 \mathrm{~g} \\
\text { d.w./g DPPH }\end{array}$ & \\
\hline & & $\mathrm{UAE}\left(60^{\circ} \mathrm{C}, 30 \mathrm{~min}, 280 \mathrm{~W}, 60 \mathrm{kHz}\right)$ & $\begin{array}{c}\text { TPC: } 20.1 \mathrm{mg} \mathrm{GAE} / \mathrm{g} ; 40.6 \mathrm{~g} \\
\text { d.w./g DPPH }\end{array}$ & \\
\hline & & HHPAE (10 min, $600 \mathrm{MPa})$ & $\begin{array}{c}\text { TPC: } 26 \text { mg GAE } / g ; 45.7 \text { g d.w./g } \\
\text { DPPH }\end{array}$ & \\
\hline \multirow{3}{*}{ Olive pomace } & $\begin{array}{c}\text { Conventional solvent } \\
\text { extraction (CSE) }\end{array}$ & CSE (Water-EtOH) & $\begin{array}{l}\text { TPC: } 16.9 \text { mg GAE/g; } \\
\text { DPPH: } 0.81 \mathrm{~g} \mathrm{TE} / \mathrm{L}\end{array}$ & \multirow{3}{*}[24]{} \\
\hline & $\begin{array}{l}\text { Ultrasound-assisted } \\
\text { extraction (UAE) }\end{array}$ & $\mathrm{UAE}\left(70^{\circ} \mathrm{C}, 120 \mathrm{~min}\right)$ & $\begin{array}{l}\text { TPC: } 14.7 \text { mg GAE/g; } \\
\text { DPPH: } 1.76 \mathrm{~g} \mathrm{TE} / \mathrm{L}\end{array}$ & \\
\hline & $\begin{array}{l}\text { Cyclodextrin-enhanced } \\
\text { pulsed UAE }\end{array}$ & Cyclodextrin-enhanced pulsed UAE & $\begin{array}{l}\text { TPC: } 69.6 \mathrm{mg} \mathrm{GAE} / \mathrm{g} \\
\text { DPPH: } 52.2 \mathrm{mg} \mathrm{TE} / \mathrm{kg}\end{array}$ & \\
\hline Olive pomace & $\begin{array}{l}\text { Ultrasound-assisted } \\
\text { extraction (UAE) }\end{array}$ & $\begin{array}{c}\text { UAE (90\% EtOH; } 50{ }^{\circ} \mathrm{C} ; 5 \mathrm{~min}, \mathrm{~S} / \mathrm{L}: 30 \\
\mathrm{~g} / \mathrm{mL}, 135.6 \mathrm{~W} / \mathrm{cm}, 60 \mathrm{kHz})\end{array}$ & $\begin{array}{c}55.1 \mathrm{mg} \mathrm{HT} / \mathrm{g} ; 381.2 \mathrm{mg} \mathrm{MA} / \mathrm{g} ; \\
29.8 \mathrm{mg} \mathrm{OA} / \mathrm{g}\end{array}$ & [25] \\
\hline Olive pomace & $\begin{array}{l}\text { Extraction via membrane } \\
\text { processing }\end{array}$ & 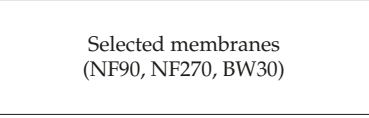 & $\begin{array}{l}\text { TPC: } 1234.3 \mathrm{mg} \text { GAE/L extract } \\
\text { TFC: } 464.2 \mathrm{mg} \text { EE/L extract } \\
\text { DPPH: } 405.9 \mu \mathrm{g} \text { TE/L extract } \\
\text { FRAP: } 9183.3 \mu \mathrm{mol} \text { FSE/L extract }\end{array}$ & [26] \\
\hline Olive cake & Conventional extraction & Methanol, $70^{\circ} \mathrm{C}, 12 \mathrm{~h}, 3$ cycles & $\begin{array}{l}\text { TPC: } 4.37 \mathrm{mg} \text { GAE } / \mathrm{g} \\
\text { DPPH: } 72 \% \text { inhibition }\end{array}$ & [27] \\
\hline Olive pomace & $\begin{array}{c}\text { Hydrothermal } \\
\text { pretreatments (steam } \\
\text { explosion (SE) and } \\
\text { subcritical water }(\mathrm{SCW}) \text { ) }\end{array}$ & $200^{\circ} \mathrm{C}, 5 \mathrm{~min}$ & $\begin{array}{c}S E \\
\text { TPC: } 73.3 \mathrm{mg} \mathrm{GAE} / \mathrm{g} \\
S C W \\
\text { TPC: } 69.7 \mathrm{mg} \mathrm{GAE} / \mathrm{g}\end{array}$ & {$[28]$} \\
\hline Olive cake & $\begin{array}{l}\text { Extraction by magnetic } \\
\text { stirring }\end{array}$ & Ethanol and acetone $(70 \%), 2 \mathrm{~h}$ & $\begin{array}{c}\text { TPC: } 63.7 \mathrm{mg} \mathrm{GAE} / \mathrm{g} \text { extract } \\
\text { DPPH: } 95.4 \% \text { inhibition } \\
\text { ORAC: } 0.82 \mathrm{mg} \mathrm{TE} / \mathrm{g}\end{array}$ & [29] \\
\hline Alperujo & Hydrothermal treatment & $\begin{array}{l}\text { Ethyl acetate, } 160{ }^{\circ} \mathrm{C}, 60 \mathrm{~min} \\
(\mathrm{pH}=4.5)\end{array}$ & $\begin{array}{l}\text { TPC: } 64.2 \mathrm{~g} \mathrm{GAE} / 100 \mathrm{~g} \\
\text { DPPH: } 12 \mathrm{mg} \mathrm{TE} / \mathrm{mL} \\
\text { FRAP: } 1.8 \mathrm{mg} \mathrm{TE} / \mathrm{mL}\end{array}$ & [30] \\
\hline Olive-waste cake & Soxhlet extraction & $\mathrm{n}$-hexane; $60 \mathrm{~min}$ & $\begin{array}{c}\text { TPC: } 35 \mathrm{mg} \mathrm{GAE} / \mathrm{g} ; \\
\text { TFC: } 13.3 \mathrm{mg} \text { CTE/g } \\
\text { DPPH: } 67.2 \mu \mathrm{mol} \mathrm{TE} / \mathrm{g} \\
\text { FRAP: } 176.7 \mu \mathrm{mol} \mathrm{TE} / \mathrm{g}\end{array}$ & {$[31]$} \\
\hline
\end{tabular}

TPC: total phenolic content; GAE: gallic acid equivalents; d.w.: dry weight; TE: trolox equivalents; TFC: total flavonoids content; HT: hydroxytyrosol; MA: maslinic acid; OA: oleanolic acid; EE: epicatechin equivalents; FSE: ferrous sulfate equivalents; CTE: catequin equivalents.

Several extraction methods have been studied, including conventional extraction, UAE, microwaveassisted extraction (MAE), high hydrostatic pressure-assisted extraction (HHPAE), supercritical fluid extraction (SFE), and extraction with eutectic solvents. In this body of work, Chanioti and Tzia [23] reported that the use of eutectic solvents improved the phenolic extraction yield from OP with respect to conventional solvents (i.e., solutions of water and ethanol). However, the content of the phenolics 
that were obtained in the present work with real EOP that was extracted by ultrasound with an ethanol-water mixture of $43 \%$ ( $57.5 \mathrm{mg}$ GAE/g EOP) was higher than that reported by these authors with the use of deep eutectic solvents and ultrasound as the extraction method (20.1 $\mathrm{mg} \mathrm{GAE} / \mathrm{g} \mathrm{OP}$ ). Caballero et al. (2018) [22] reported a TPC of $14.1 \mathrm{mg}$ GAE from exhausted pomace while using SFE. Seçmeler et al. (2018) [28] proposed the use of steam explosion and other hydrothermal pretreatments, such as subcritical water for the recovery of phenols and reported higher values (69.7-73.3 mg GAE) than those that were obtained in the present research. Regarding the content of specific phenolic compounds, Xie et al., [25] found that UAE resulted in greater extraction efficiencies of hydroxytyrosol, maslinic acid, and oleanolic acid from OP when compared with conventional extraction and MAE. Importantly, the comparison of the results from different works that utilised different extraction techniques is difficult due to the variability of OP and EOP samples (e.g., differences due to the type of cultivation and the variety and maturation of the olives) and the different methods that are used to quantify the phenolic compound content and measure the antioxidant activity. However, the results that were obtained here and in the studies reported in the bibliography suggest that the residual biomass contains noticeable amounts of phenols and high antioxidant activity. Therefore, the extract from olive pomace has potential for use as a natural bioactive ingredient in different industrial applications.

\section{Conclusions}

Exhausted olive pomace is an interesting source of natural antioxidant compounds, which can be extracted as a first step in a biorefinery strategy for valorisation of this agro-industrial residue. This work investigated the impact of ultrasound assisted extraction conditions on TPC, TFC, and antioxidant activity of EOP while using RSM. The optimal conditions for UAE were determined at $43.2 \%$ ethanol concentration, 70\% ultrasound amplitude, and 15 min extraction time, yielding an optimal extract with higher phenolic and flavonoid content, as well as higher antioxidant activity than those previously determined for other by-products of the olive oil industries, such olive oil or olive tree pruning also extracted by ultrasound.

Author Contributions: I.R., E.R. and E.C. designed the experiments and revised the manuscript; J.C.M.-P. and I.G.-C. performed the experimental part and wrote the draft version of the manuscript; M.B., B.G. and I.R. supervised the experimental work and analysed and discussed the results. All authors approved the final version.

Funding: This research was funded by the Spanish Ministerio de Economía y Competitividad- Agencia Estatal de Investigación and Fondo Europeo de Desarrollo Regional. Project Reference ENE2017-85819-C2-1-R.

Acknowledgments: José Carlos Martínez Patiño gratefully acknowledges financial support provided by the Universidad de Jaén Doctoral School. Irene Gómez-Cruz expresses her gratitude also to the Universidad de Jaén for financial support (grant R5/04/2017).

Conflicts of Interest: The authors declare no conflict of interest.

\section{References}

1. Medina-Torres, N.; Ayora-Talavera, T.; Espinosa-Andrews, H.; Sánchez-Contreras, A.; Pacheco, N. Ultrasound assisted extraction for the recovery of phenolic compounds from vegetable sources. Agronomy 2017, 7, 47. [CrossRef]

2. Gullón, B.; Gullón, P.; Lú-Chau, T.A.; Moreira, M.T.; Lema, J.M.; Eibes, G. Optimization of solvent extraction of antioxidants from Eucalyptus globulus leaves by response surface methodology: Characterization and assessment of their bioactive properties. Ind. Crops Prod. 2017, 108, 649-659. [CrossRef]

3. Gullón, B.; Eibes, G.; Moreira, M.T.; Herrera, R.; Labidi, J.; Gullón, P. Yerba mate waste: A sustainable resource of antioxidant compounds. Ind. Crop. Prod. 2018, 113, 398-405. [CrossRef]

4. Romero-García, J.M.; Niño, L.; Martínez-Patiño, C.; Álvarez, C.; Castro, E.; Negro, M.J. Biorefinery based on olive biomass. State of the art and future trends. Bioresour. Technol. 2014, 159, 421-432. [CrossRef] [PubMed]

5. Food and Agriculture Organization of the United Nations. FAOSTAT. 2019. Available online: http://faostat3. fao.org/ (accessed on 3 May 2019). 
6. Manzanares, P.; Ruiz, E.; Ballesteros, M.; Negro, M.J.; Gallego, F.J.; López-Linares, J.C.; Castro, E. Residual biomass potential in olive tree cultivation and olive oil industry in Spain: Valorization proposal in a biorefinery context. Span. J. Agric. Res. 2017, 15, 6. [CrossRef]

7. Ruiz, E.; Romero-García, J.M.; Romero, I.; Manzanares, P.; Negro, M.J.; Castro, E. Olive-derived biomass as a source of energy and chemicals. Biofuel Bioprod. Biorefining 2017, 11, 1077-1094. [CrossRef]

8. Berbel, J.; Posadillo, A. Review and analysis of alternatives for the valorisation of agro-industrial olive oil by-products. Sustainability 2018, 10, 237. [CrossRef]

9. Pérez-Bonilla, M.; Salido, S.; Van Beek, T.A.; Waard, P.D.; Linares-Palomino, P.J.; Sánchez, A.; Altarejos, J. Isolation of antioxidative secoiridoids from olive wood (Olea europaea L.) guided by on-line HPLC-DAD-radical scavenging detection. Food Chem. 2011, 124, 36-41. [CrossRef]

10. Gullón, B.; Gullón, P.; Eibes, G.; Cara, C.; De Torres, A.; López-Linares, J.C.; Ruiz, E.; Castro, E. Valorisation of olive agro-industrial by-products as a source of bioactive compounds. Sci. Total Environ. 2018, 645, 533-542. [CrossRef]

11. Hayta, M.; Isçimen, E.M. Optimization of ultrasound-assisted antioxidant compounds extraction from germinated chickpea using response surface methodology. LWT Food Sci. Technol. 2017, 77, $208-216$. [CrossRef]

12. Khemakhem, I.; Ahmad-Qasem, M.H.; Catalán, E.B.; Micol, V.; García-Pérez, J.V.; Ayadi, M.A.; Bouaziz, M. Kinetic improvement of olive leaves bioactive compounds extraction by using power ultrasound in a wide temperature range. Ultrason. Sonochem. 2017, 34, 466-473. [CrossRef] [PubMed]

13. Hosseini, H.; Bolourian, S.; Yaghoubi Hamgini, E.; Ghanuni Mahababadi, E. Optimization of heatand ultrasound-assisted extraction of polyphenols from dried rosemary leaves using response surface methodology. J. Food Process. Preserv. 2018, 42, e13778. [CrossRef]

14. Tiwari, B.K. Ultrasound: A clean, green extraction technology. TrAC Trends Anal. Chem. 2015, 71, 100-109. [CrossRef]

15. Bamba, B.S.B.; Shi, J.; Tranchant, C.C.; Xue, S.J.; Forney, C.F.; Lim, L.T. Influence of extraction conditions on ultrasound-assisted recovery of bioactive phenolics from blueberry pomace and their antioxidant activity. Molecules 2018, 23, 1685. [CrossRef] [PubMed]

16. Singleton, V.L.; Rossi, S.A. Colorimetric of total phenolics with phosphomolibic-phosphotungstic acid reagents. Am. J. Enol. Vitic. 1965, 16, 144-158.

17. Martínez-Patiño, J.C.; Gullón, B.; Romero, I.; Ruiz, E.; Brncic, M.; Žlabur, J.Š.; Castro, E. Optimization of ultrasound-assisted extraction of biomass from olive trees using response surface methodology. Ultrason. Sonochem. 2019, 51, 487-495. [CrossRef]

18. Kim, D.O.; Jeong, S.W.; Lee, C.Y. Antioxidant capacity of phenolic phytochemicals from various cultivars of plums. Food Chem. 2003, 81, 321-326. [CrossRef]

19. Goldsmith, C.D.; Vuong, Q.V.; Stathopoulos, C.E.; Roach, P.D.; Scarlett, C.J. Ultrasound increases the aqueous extraction of phenolic compounds with high antioxidant activity from olive pomace. LWT Food Sci. Technol. 2018, 89, 284-290. [CrossRef]

20. Zardo, I.; de Espíndola Sobczyk, A.; Marczak, L.D.F.; Sarkis, J. Optimization of Ultrasound Assisted Extraction of Phenolic Compounds from Sunflower Seed Cake Using Response Surface Methodology. Waste Biomass Valoris. 2019, 10, 33-44. [CrossRef]

21. Papoutsis, K.; Pristijono, P.; Golding, J.B.; Stathopoulos, C.E.; Bowyer, M.C.; Scarlett, C.J.; Vuong, Q.V. Optimizing a sustainable ultrasound-assisted extraction method for the recovery of polyphenols from lemon by-products: Comparison with hot water and organic solvent extractions. Eur. Food Res. Technol. 2018, 244, 1353-1365. [CrossRef]

22. Caballero, A.S.; Romero-García, J.M.; Castro, E.; Cardona, C.A. Supercritical fluid extraction for enhancing polyphenolic compounds production from olive waste extracts. J. Chem. Technol. Biotechnol. 2019. Available online: https://onlinelibrary.wiley.com/doi/abs/10.1002/jctb.5907/ (accessed on 3 May 2019). [CrossRef]

23. Chanioti, S.; Tzia, C. Extraction of phenolic compounds from olive pomace by using natural deep eutectic solvents and innovative extraction techniques. Innov. Food Sci. Emerg. Technol. 2018, 48, 228-239. [CrossRef]

24. Albahari, P.; Jug, M.; Radic, K.; Jurmanovic, S.; Brncic, M.; Brncic, S.R.; Vitali Cepo, D. Characterization of olive pomace extract obtained by cyclodextrin-enhanced pulsed ultrasound assisted extraction. LWT Food Sci. Technol. 2018, 92, 22-31. [CrossRef] 
25. Xie, P.; Huang, L.; Zhang, C.; Deng, Y.; Wang, X.; Cheng, J. Enhanced extraction of hydroxytyrosol, maslinic acid and oleanolic acid from olive pomace: Process parameters, kinetics and thermodynamics, and greenness assessment. Food Chem. 2019, 276, 662-674. [CrossRef] [PubMed]

26. Antónia Nunes, M.; Pawlowski, S.; Costa, A.S.G.; Alves, R.C.; Oliveira, M.B.P.P.; Velizarov, S. Valorization of olive pomace by a green integrated approach applying sustainable extraction and membrane-assisted concentration. Sci. Total Environ. 2019, 652, 40-47. [CrossRef] [PubMed]

27. Alu'datt, M.H.; Alli, I.; Ereifej, K.; Alhamad, M.; Al-Tawaha, A.R.; Rababah, T. Optimisation, characterisation and quantification of phenolic compounds in olive cake. Food Chem. 2010, 123, 117-122. [CrossRef]

28. Seçmeler, Ö.; Güçlü Üstündag, Ö.; Fernández-Bolaños, J.; Rodríguez-Gutiérrez, G. Effect of subcritical water and steam explosion pretreatments on the recovery of sterols, phenols and oil from olive pomace. Food Chem. 2018, 265, 298-307. [CrossRef]

29. Moudache, M.; Colon, M.; Nerín, C.; Zaidi, F. Phenolic content and antioxidant activity of olive by-products and antioxidant film containing olive leaf extract. Food Chem. 2016, 212, 521-527. [CrossRef] [PubMed]

30. Rubio-Senent, F.; Fernández-Bolaños, J.; García-Borrego, A.; Lama-Muñoz, A.; Rodríguez-Gutiérrez, G. Influence of $\mathrm{pH}$ on the antioxidant phenols solubilised from hydrothermally treated olive oil by-product (alperujo). Food Chem. 2017, 219, 339-345. [CrossRef]

31. Uribe, E.; Pasten, A.; Lemus-Mondaca, R.; Vega-Gálvez, A.; Quispe-Fuentes, I.; Ortiz, J.; Di Scala, K. Comparison of Chemical Composition, Bioactive Compounds and Antioxidant Activity of Three Olive-Waste Cakes. J. Food Biochem. 2015, 39, 189-198. [CrossRef]

(C) 2019 by the authors. Licensee MDPI, Basel, Switzerland. This article is an open access article distributed under the terms and conditions of the Creative Commons Attribution (CC BY) license (http://creativecommons.org/licenses/by/4.0/). 

Article

Monitoring the Bioactive Compounds Status in Olea europaea According to Collecting Period and Drying Conditions

\author{
Amani Taamalli ${ }^{1}$, Jesus Lozano Sánchez ${ }^{2,3, *}$, Haifa Jebabli ${ }^{1}$, Najla Trabelsi ${ }^{1}$, Leila Abaza ${ }^{1}$, \\ Antonio Segura Carretero ${ }^{3,4}$, Jae Youl Cho ${ }^{5}$ and David Arráez Román ${ }^{3,4}$ \\ 1 Laboratoire de Biotechnologie de l'Olivier, Centre de Biotechnologie de Borj-Cedria, \\ B/P 901 Hammam-Lif 2050, Tunisia; taamalli_ameni@yahoo.fr (A.T.); jebebli@hotmail.com (H.J.); \\ najla.trabelsi@cbbc.rnrt.tn (N.T.); leila.abaza@cbbc.rnrt.tn (L.A.) \\ 2 Department of Food Science and Nutrition, University of Granada, Campus Universitario s/n, \\ 18071 Granada, Spain \\ 3 Center of Research and Development of Functional Food, Health Science Technological Park, \\ Avda. del Conocimiento s/n, 18100 Granada, Spain; ansegura@ugr.es (A.S.C.); darraez@ugr.es (D.A.R.) \\ 4 Department of Analytical Chemistry, Faculty of Sciences, University of Granada, 18071 Granada, Spain \\ 5 Department of Integrative Biotechnology, Sungkyunkwan University, 2066 Seobu-ro, Suwon 16419, Korea; \\ jaecho67@gmail.com \\ * Correspondence: jesusls@ugr.es
}

Received: 4 January 2019; Accepted: 6 March 2019; Published: 12 March 2019

\begin{abstract}
Polyphenols and triterpenoids in olive have relevant importance both in the physiology of the plant and the nutritional and biological value of its products. Olive leaf extracts are of special interest for their numerous health-promoting properties. The present research is investigating the occurrence of phytochemicals in supercritical fluid extracts from leaves with regard to collection time and drying temperature. The phytochemical profiles of the olive leaf extracts were determined by reversed-phase high-performance liquid chromatography (HPLC) coupled to electrospray ionization time-of-flight mass spectrometry (ESI-TOF-MS) detection. The main extracted phytochemicals were phenols and terpenoids. A significant variation in the amounts of the different components was observed as a function of the different drying temperature and collecting time $(p<0.05)$. Among samples, the maximal contents of polyphenols and secoiridoid derivatives were found in the extracts from olive leaves collected in November and dried at $120^{\circ} \mathrm{C}$, whereas triterpenoids showed the highest content in fresh leaves collected in August.
\end{abstract}

Keywords: Olea europaea; supercritical fluid extraction; triterpenoids; polyphenols; olive leaves

\title{
1. Introduction
}

The incidence of many chronic disorders, such as cardiovascular diseases and certain types of cancers, could be attenuated by an improved diet, particularly through increased consumption of diets rich in fruits and vegetables. Such health-promoting properties of edible plants are related mainly to the presence of secondary metabolites, known as phytochemicals [1]. In contrast to traditional pharmaceutical drugs based on single defined substances, herbal preparations have been characterized by a multi-ingredient nature. However, recently this strategy has been introduced in modern medicine with the use of multi-ingredient pharmaceutical cocktails, now prevalent in the treatment of some diseases [2].

The exploitation of olive leaves as a natural resource rich in bioactive compounds would involve the valorization of this byproduct [3]. In addition, olive leaves have a high potential for exploitation 
in the food industry. Properties of olive leaves are generally attributed to the presence of a range of triterpenes and phenolic compounds such as secoiridoids, lignans, flavonoids, etc. [4].

Fresh olive leaves generally need drying and milling before any purpose. As a preservation method, drying is carried out to remove the water from the leaves to protect the leaves against spoilage and degradation of oleuropein by enzyme action. It also improves extraction efficiency or extractability [5]. Thus, it is considered as the main process in olive leaf treatment. Olive leaves have to be dried for use as an ingredient in dry mixes, extracting phenolic compounds having antioxidant properties, and use in olive leaf tea [6]. In fact, the immediate drying of olive leaves is the most important operation in post-harvest processing in order to avoid quality losses and prevent possible degradation during storage [7], since drying might affect the product quality and is an energy-intensive process [8].

Thus, this work has focused on the determination of phenolic compounds and triterpenoids from fresh and dried olive leaves from El Hor cultivar, cultivated in the center of Tunisia at four sampling dates (January, April, August, and November) using an HPLC-ESI-TOF MS platform. The choice of this olive cultivar was based on our previous findings demonstrating that El Hor olive leaves extracted by supercritical fluid extraction showed the best anticancer activity among other cultivars and extraction techniques $[9,10]$. Thus, for in-depth study, the aim of this work was to study how the drying process and collecting period of olive leaves affect the phenolic composition of extracts in order to obtain extracts rich in bioactive compounds.

\section{Results and Discussion}

\subsection{Identification of Phytochemicals in Olive Leaf Extracts}

Natural antioxidants are mainly secondary metabolites such as phenolic acids, flavonoids, and terpenoids, which are produced by plants for sustaining growth under adverse environment [11]. Polyphenols are among the most widespread class of secondary metabolites in nature, which possess an aromatic ring with one or more hydroxyl substituents.

In the present study, olive leaf extracts were analyzed by HPLC with TOF/MS detection. The characterization process was conducted using the elution order, the interpretation of their mass spectrum provided by the TOF-MS, commercial standards when available, and the data previously reported in the literature. Table 1 includes the compounds, which were identified in olive leaves' SFE extracts, and the information generated by the TOF analyzer-retention time, experimental and calculated $\mathrm{m} / \mathrm{z}$, molecular formula, and error and milliSigma value. A total of 20 compounds were characterized in SFE extracts by the HPLC-ESI-TOF/MS analytical methods described above. Among them, 17 compounds were from different polar compound classes and polyphenolic families. We classify them into groups such as secoiridoids and related derivatives, simple phenolic compounds, flavonoids (flavonols, flavones, and O-methylated flavones), and lignans. In addition, three were characterized as triterpenoids. 
Table 1. Mass spectral data of the phytochemicals identified in the olive leaf extracts.

\begin{tabular}{|c|c|c|c|c|c|c|c|}
\hline Peak & $m / z$ & R.T. (min) & $\begin{array}{l}\text { Molecular } \\
\text { Formula }\end{array}$ & $\begin{array}{r}\text { Error } \\
(\mathrm{ppm})\end{array}$ & mSigma & Compound Name & Group \\
\hline 1 & 389.1089 & 8.25 & $\mathrm{C}_{16} \mathrm{H}_{22} \mathrm{O}_{11}$ & 3.1 & 1 & Secologanoside * & $\begin{array}{l}\text { Secoiridoids and } \\
\text { related derivatives }\end{array}$ \\
\hline 2 & 153.0557 & 8.90 & $\mathrm{C}_{8} \mathrm{H}_{10} \mathrm{O}_{3}$ & -0.5 & 2 & Hydroxytyrosol $^{\#}$ & $\begin{array}{l}\text { Simple phenols: } \\
\text { Phenyl alcohols }\end{array}$ \\
\hline 3 & 389.1447 & 10.33 & $\mathrm{C}_{16} \mathrm{H}_{22} \mathrm{O}_{11}$ & -0.5 & 4.4 & Loganoside * & Secoiridoids \\
\hline 4 & 401.1453 & 11.10 & $\mathrm{C}_{18} \mathrm{H}_{26} \mathrm{O}_{10}$ & 3.8 & 2.7 & $\begin{array}{l}\text { benzyl alcohol } \\
\text { pentose * }\end{array}$ & $\begin{array}{l}\text { Other polar } \\
\text { compounds }\end{array}$ \\
\hline 5 & 403.1246 & 11.30 & $\mathrm{C}_{17} \mathrm{H}_{24} \mathrm{O}_{11}$ & 2.8 & 6.9 & $\begin{array}{c}\text { Elenolic acid } \\
\text { glucoside isomer } 1 *\end{array}$ & $\begin{array}{l}\text { Secoiridoids and } \\
\text { related derivatives }\end{array}$ \\
\hline 6 & 151.0401 & 11.70 & $\mathrm{C}_{8} \mathrm{H}_{8} \mathrm{O}_{3}$ & -4.8 & 6.4 & vanillin \# & $\begin{array}{l}\text { Simple phenols: } \\
\text { Aldehydes }\end{array}$ \\
\hline 7 & 403.1246 & 12.09 & $\mathrm{C}_{17} \mathrm{H}_{24} \mathrm{O}_{11}$ & -0.5 & 13.8 & $\begin{array}{c}\text { Elenolic acid } \\
\text { glucoside isomer } 2 *\end{array}$ & $\begin{array}{l}\text { Secoiridoids and } \\
\text { related derivatives }\end{array}$ \\
\hline 8 & 193.0506 & 14.63 & $\mathrm{C}_{10} \mathrm{H}_{10} \mathrm{O}_{4}$ & 3.3 & 9.4 & Ferulic acid \# & $\begin{array}{l}\text { Simple phenols: } \\
\text { Hydroxycinnamic } \\
\text { acids }\end{array}$ \\
\hline 9 & 539.1770 & 15.46 & $\mathrm{C}_{25} \mathrm{H}_{32} \mathrm{O}_{13}$ & 5.2 & 3.8 & Oleuropein isomer $1^{\#}$ & $\begin{array}{l}\text { Secoiridoids and } \\
\text { related derivatives }\end{array}$ \\
\hline 10 & 539.1770 & 15.96 & $\mathrm{C}_{25} \mathrm{H}_{32} \mathrm{O}_{13}$ & 4.7 & 2.4 & Oleuropein isomer $2^{\#}$ & $\begin{array}{l}\text { Secoiridoids and } \\
\text { related derivatives }\end{array}$ \\
\hline 11 & 523.1821 & 23.92 & $\mathrm{C}_{25} \mathrm{H}_{31} \mathrm{O}_{12}$ & 4.0 & 4.8 & Ligstroside * & $\begin{array}{c}\text { Secoiridoids and } \\
\text { related derivatives }\end{array}$ \\
\hline 12 & 417.1555 & 18.59 & $\mathrm{C}_{22} \mathrm{H}_{26} \mathrm{O}_{8}$ & -2.0 & 35.6 & Syringaresinol $^{\#}$ & Lignans \\
\hline 13 & 285.0405 & 19.00 & $\mathrm{C}_{15} \mathrm{H}_{10} \mathrm{O}_{6}$ & & & Luteolin ${ }^{\#}$ & Flavonoids: Flavones \\
\hline 14 & 301.0354 & 19.20 & $\mathrm{C}_{15} \mathrm{H}_{10} \mathrm{O}_{7}$ & 1.9 & 11.24 & Quercetin ${ }^{\#}$ & Flavonoids: Flavonols \\
\hline 15 & 357.1344 & 19.9 & $\mathrm{C}_{20} \mathrm{H}_{22} \mathrm{O}_{6}$ & 1.2 & 14.9 & pinoresinol $^{\#}$ & Lignans \\
\hline 16 & 415.1398 & 20.56 & $\mathrm{C}_{22} \mathrm{H}_{24} \mathrm{O}_{8}$ & -2.6 & 9.6 & acetoxypinoresinol \# & Lignans \\
\hline 17 & 299.0561 & 22.97 & $\mathrm{C}_{16} \mathrm{H}_{12} \mathrm{O}_{6}$ & 9.3 & 14.2 & diosmetin \# & $\begin{array}{l}\text { Flavonoids: } \\
\text { O-methylated } \\
\text { flavones }\end{array}$ \\
\hline Peak & $m / z$ & R.T. (min) & $\begin{array}{l}\text { Molecular } \\
\text { Formula }\end{array}$ & $\begin{array}{r}\text { Error } \\
(\mathrm{ppm})\end{array}$ & mSigma & Compound Name & Class \\
\hline 18 & 471.3480 & 14.71 & $\mathrm{C}_{30} \mathrm{H}_{48} \mathrm{O}_{4}$ & 14.3 & 5.8 & Maslinic acid $^{\#}$ & triterpenoids \\
\hline 19 & 455.3531 & 16.30 & $\mathrm{C}_{30} \mathrm{H}_{48} \mathrm{O}_{3}$ & 11.3 & 3.4 & Oleanolic acid \# & triterpenoids \\
\hline 20 & 455.3531 & 16.41 & $\mathrm{C}_{30} \mathrm{H}_{48} \mathrm{O}_{3}$ & 12.1 & 8.8 & Ursolic acid * & triterpenoids \\
\hline
\end{tabular}

* Identified on the basis of mass spectra. Comparison with the literature, \# Identified on the basis of external pure standard.

Figure 1 shows the resulting base peak chromatograms (BPCs) of fresh and dried olive leaf samples collected in January from A1 to A7 according to drying temperature. The main phytochemicals identified are included in Table 1, and their corresponding extracted ion chromatograms (EICs) are shown in Figure 2. 

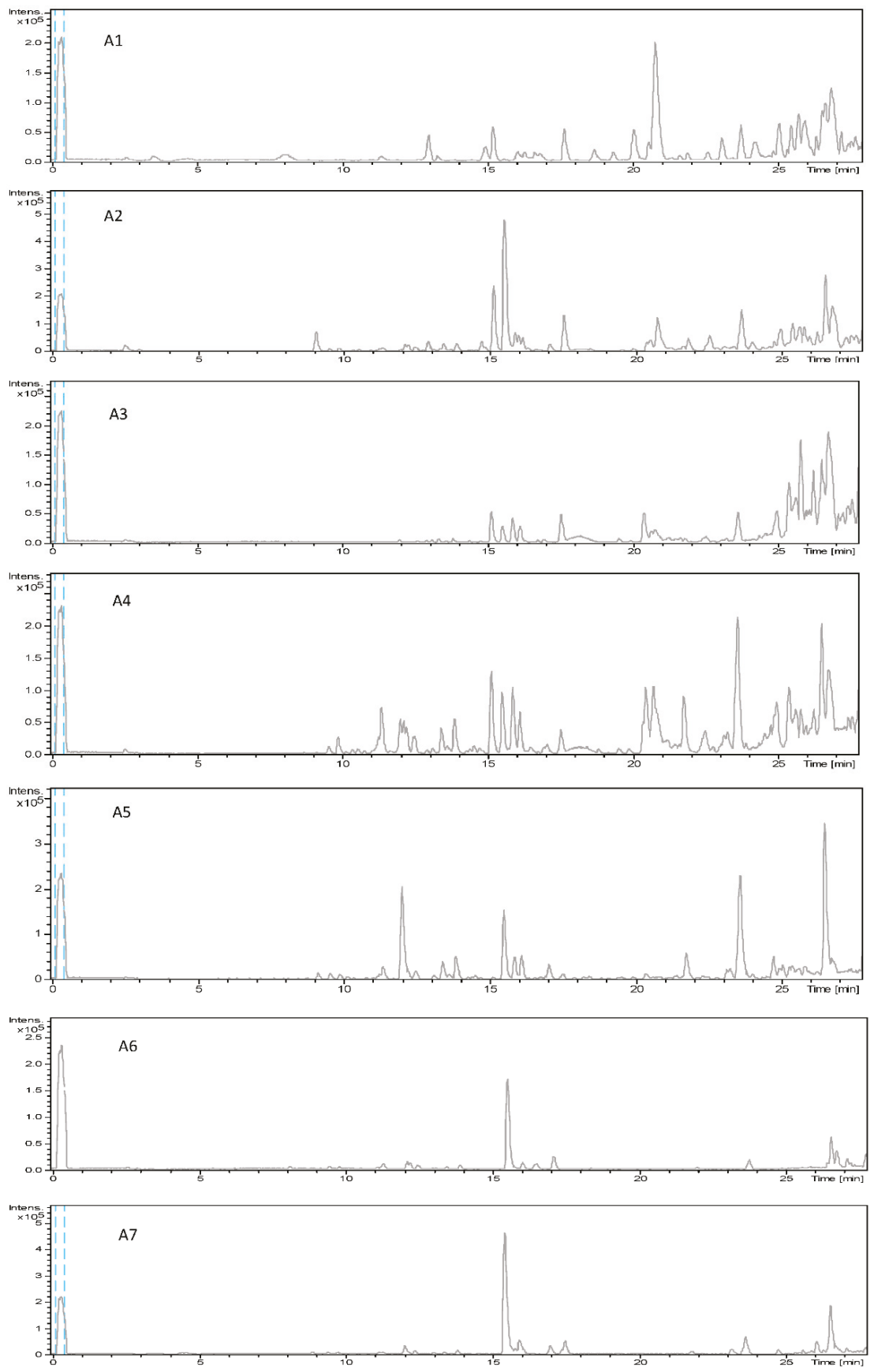

Figure 1. Base peak chromatograms (BPCs) of fresh and dried olive leaf samples collected in January from A1 to A7 according to drying temperature. A1: Fresh leaves, A2: leaves dried at $25^{\circ} \mathrm{C}$, A3: leaves dried at $40{ }^{\circ} \mathrm{C}$, A4: leaves dried at $60^{\circ} \mathrm{C}$, A5: leaves dried at $80^{\circ} \mathrm{C}$, A6: leaves dried at $100{ }^{\circ} \mathrm{C}, \mathrm{A} 7$ : leaves dried at $120^{\circ} \mathrm{C}$. 

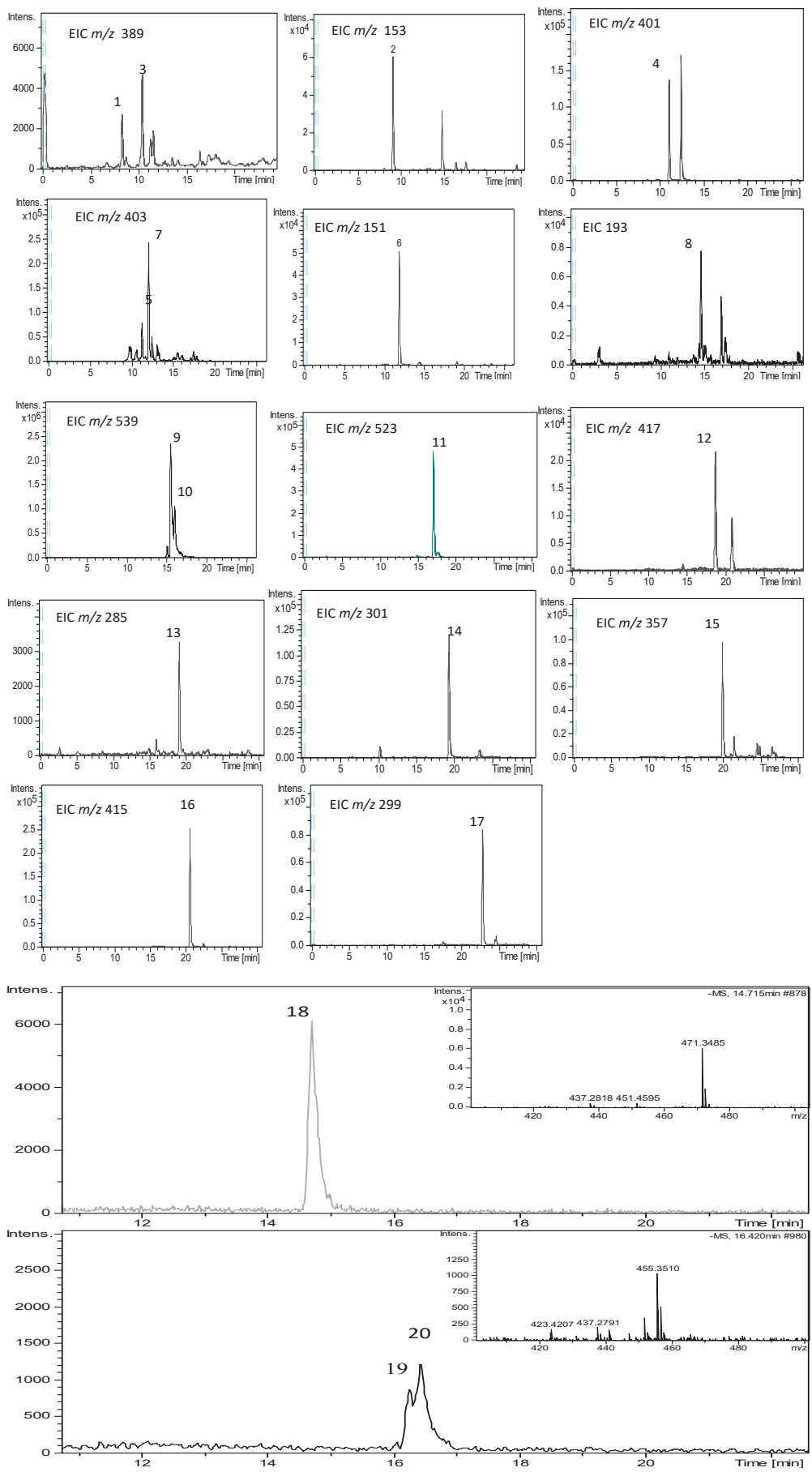

Figure 2. Extracted ion chromatograms (EICs) of identified compounds in olive leaf samples. 


\subsubsection{Simple Phenolic Compounds}

One phenyl alcohol (peak 2 eluted at $8.9 \mathrm{~min}$ ), aldehyde (peak 6 eluted at $11.7 \mathrm{~min}$ ), and hydroxycinnamic acid (peak 8 eluted at $14.63 \mathrm{~min}$ ) were identified in our extracts as hydroxytyrosol, vanillin, and ferulic acid, respectively. The identification of these compounds was confirmed with their corresponding analytical standards.

\subsubsection{Secoiridoids}

Secoiridoids are produced from the secondary metabolism of terpenes. These compounds are characterized by the presence of elenolic acid or its derivates in its glycosidic or aglyconic form in their molecular structure [12]. As reported in Table 1, secoiridoids that could be identified in the different extracts included free forms such as secologanoside (peak $1, \mathrm{~m} / \mathrm{z} 389.1089$ ) and loganoside (peak $1, \mathrm{~m} / \mathrm{z}$ 389.1447), as well as phenol-conjugated secoiridoids in glycosidic form such as oleuropein (peaks 9 and 10, $m / z$ 539.177) and ligstroside (peak 11, $m / z$ 523.1821). Such compounds have been reported in olive leaves and other organs of the olive tree in previous literature [13-16].

\subsubsection{Flavonoids}

Flavones, flavonols, and $\mathrm{O}$-methylated flavones were the main flavonoids identified in the olive leaf extracts according to sampling time and drying. These were luteolin with an ion at $m / z 285.0405$ (peak 13), quercetin with an ion at $m / z 301.0354$ (peak 14), and diosmetin with an ion at $m / z 299.0561$ (peak 17). The identification of these flavonoids was based on their analytical standards together with their MS spectra. They have also been reported in previous literature [13].

\subsubsection{Lignans}

Three lignans compounds could be identified in the different olive leaf extracts under study. Peaks 12, 15, and 16 showed ions at $m / z$ 417.1555, 357.1344, and 415.1398, respectively, that were determined as syringaresinol, pinoresinol, and acetoxypinoresinol, respectively. These lignans were also reported in our previous work on fresh leaves [13].

\subsubsection{Other Polar Compounds}

Peak 4 with an ion at $\mathrm{m} / \mathrm{z} 401.1453$ eluted at $11.10 \mathrm{~min}$ and was identified as benzyl alcohol pentose. This polar compound was reported in olive leaves in previous literature [17] as well as in other plant extracts $[18,19]$.

\subsubsection{Triterpenoids}

Triterpenic compounds are common constituents of plants, occurring in the form of free acids or aglycones of triterpenoid saponins [20] offering a wide range of health-promoting activities, both as pure substances or as blends [21]. Despite their beneficial properties, there is relatively little information available on their distribution in olive leaves. To our knowledge, this is the first report on their concentration in olive leaves in response to drying and season. In this work, three triterpenic acids could be identified in the different olive leaf extracts. As demonstrated in Figure 2, peak 18 showed an ion at $\mathrm{m} / \mathrm{z} 471.3480$ eluting at $14.71 \mathrm{~min}$ which was identified as maslinic acid. Peaks that eluted at 16.30 and $16.45 \mathrm{~min}$ gave ions at $\mathrm{m} / \mathrm{z} 455.3531$ and were identified as oleanolic acid and ursolic acid, respectively (Figure 2).

\subsection{Quantification of Phytochemicals in Olive Leaf Extracts}

The phytochemicals were quantified in the different olive leaf extracts using the analytical standard calibration curves. Figure 3 shows the variation of the determined amounts of the identified phytochemicals. For phenolic compounds, oleuropein and derivatives were quantified using the calibration curve of the external standard oleuropein. Hydroxytyrosol, ferulic acid, pinoresinol, 
luteolin, apigenin, diosmetin, and quercetin were quantified using their corresponding standard calibration curves. Acetoxypinoresinol and syringaresinol were quantified using the calibration curve of pinoresinol. For triterpenoids, oleanolic acid and ursolic acid were quantified with the calibration curve of oleanolic acid, whereas maslinic acid was quantified with the calibration curve of maslinic acid. Finally, benzyl alcohol pentose was quantified using the calibration curve of hydroxytyrosol.

2.2.1. Variation of Phenolic and Triterpenoid Compounds Depending on Sampling Time and Drying Temperature

Phenolic and triterpenic compounds were determined, and their amounts expressed as $\mu \mathrm{g} / \mathrm{g}$ of olive leaf are summarized in Figure 3. Significant variation ( $p<0.05$, Supplementary Materials) was observed among samples according to the drying temperature and sampling season.

Effect of Sampling Time

The seasonal changes in the composition of the leaves of the El Hor olive cultivar have been investigated with respect to sampling time: January, April, August, and November. The quantity and distribution of phenolic and triterpenic compounds in the leaves showed significant differences among samples of different seasons $(p<0.05)$.

As shown in Figure 4, contents in secoiridoids varied significantly among sampling time $(p<0.05)$. In fresh leaves, the highest amount was observed in leaves collected in summer and did not exceed $1 \mu \mathrm{g} / \mathrm{g}$. However, for dried leaves, the highest amounts were registered for leaves collected in November $(338.8 \mu \mathrm{g} / \mathrm{g})$ except for leaves dried at $25^{\circ} \mathrm{C}$. In the latter, the January sampling time presented the highest amounts $(24.5 \mu \mathrm{g} / \mathrm{g})$ which decreased to $0.6 \mu \mathrm{g} / \mathrm{g}$ in August to slightly increase in November to reach $6.52 \mu \mathrm{g} / \mathrm{g}$. As reported by other researchers, the contents of oleuropein in olive leaves were in general barely influenced by the collecting period factor [22].

It was reported that December was the sampling time during which oleuropein presented the maximum amount for 'Leccino' cultivar [4]. In another work, the samples of leaves collected in October seemed to contain slightly lower amounts of oleuropein than those collected in March, likely due to a higher degradation rate of this glycoside in autumn and in addition to lower production of green young leaves (leaf renovation) during this season with respect to the spring period [22].

The variations in lignans amounts observed in olive leaves according to sampling time were affected by the drying temperature. In fact, fresh leaves did not show the same behavior as observed in dried leaves. As shown in Figure 4, lignans in fresh leaves decreased from January $(12.6 \mu \mathrm{g} / \mathrm{g})$ to April $(4.0 \mu \mathrm{g} / \mathrm{g})$ and re-increased in August $(9.1 \mu \mathrm{g} / \mathrm{g})$ and decreased in November, to reach values similar to those observed in April $(4.2 \mu \mathrm{g} / \mathrm{g})$. However, in leaves dried at 25 and $60^{\circ} \mathrm{C}$, a slight increase was observed from January to April in contrast to leaves dried at $40{ }^{\circ} \mathrm{C}$, where lignans amounts, after a decrease from January to April, increased in August and November to reach $2.41 \mu \mathrm{g} / \mathrm{g}$. As far as we know, this is the first time the behavior of lignans has been studied according to drying.

Luteolin and quercetin were detected only in the November sampling time, whereas diosmetin was detected in samples from each period. Flavonoids amounts showed significant variations $(p<0.05)$ among samples, however, they did not exceed $3.1 \mu \mathrm{g} / \mathrm{g}$ according to the sampling time. The highest values were observed in August and November for fresh leaves and when leaves were dried at $120^{\circ} \mathrm{C}$ (Figure 4). 

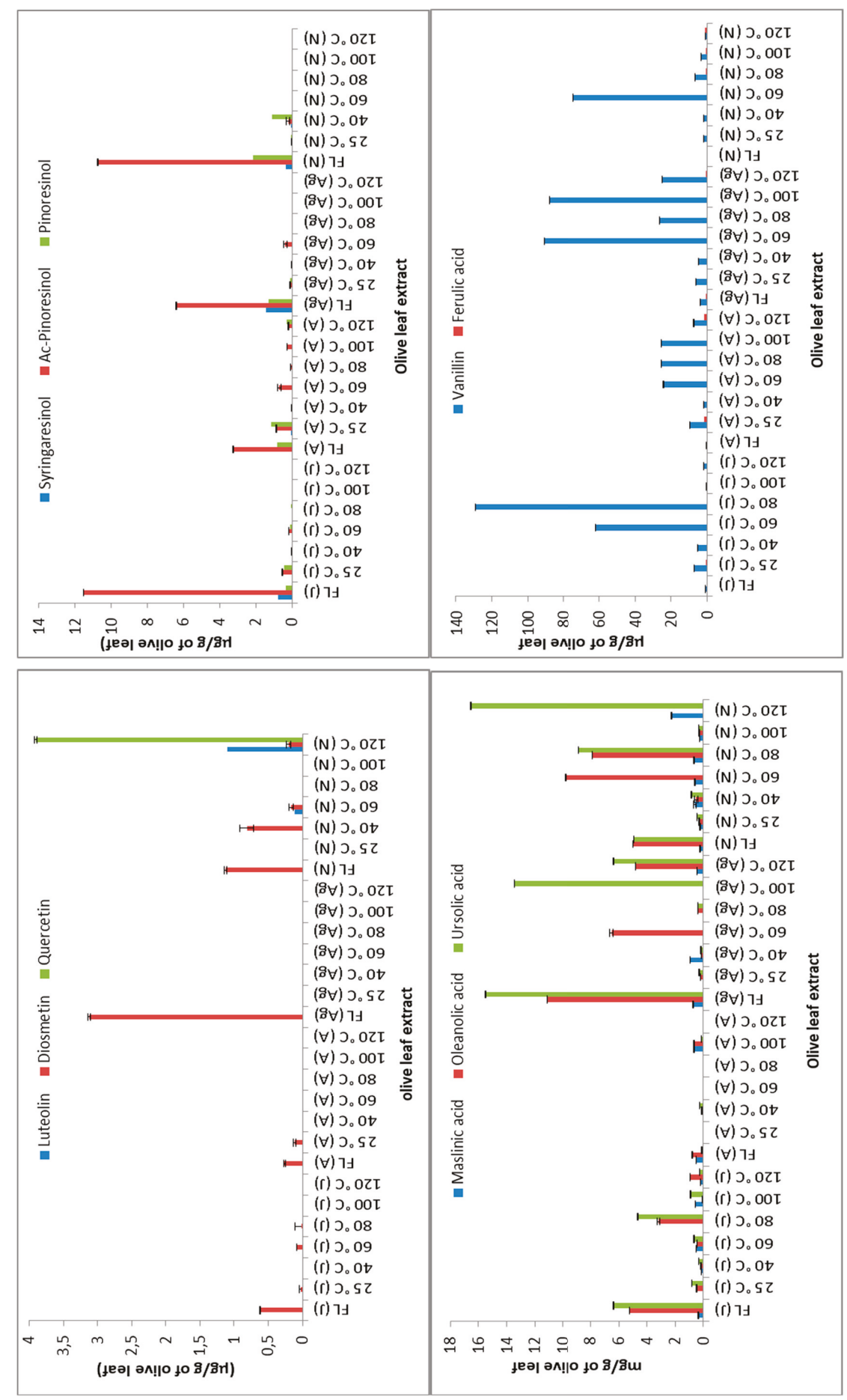

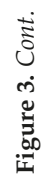



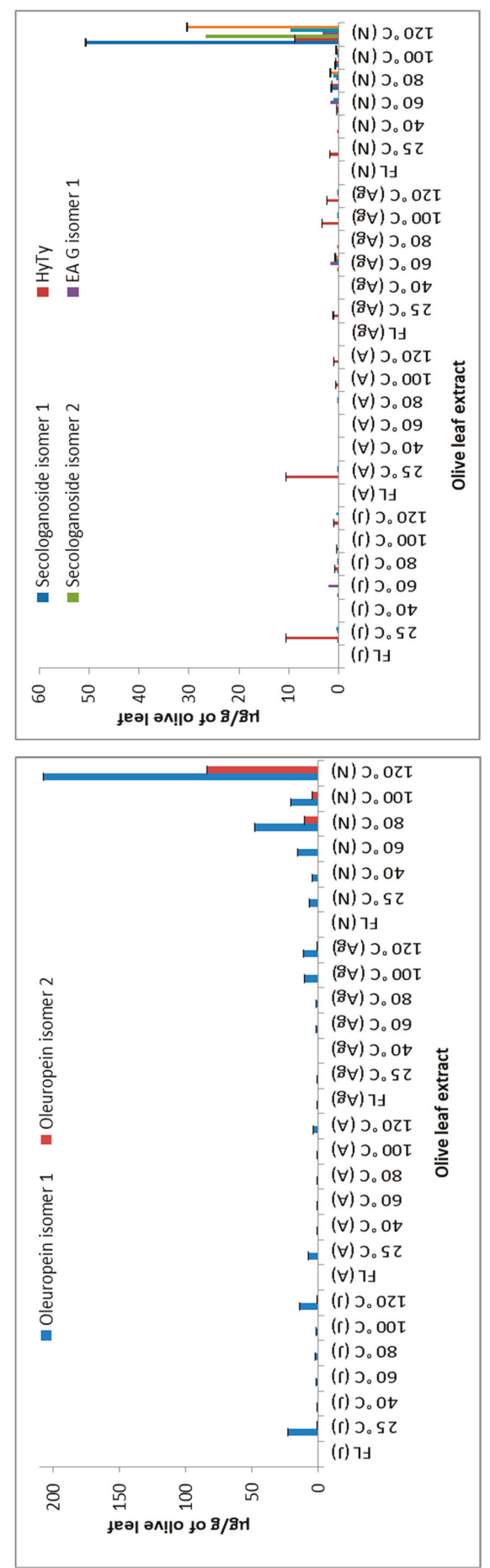

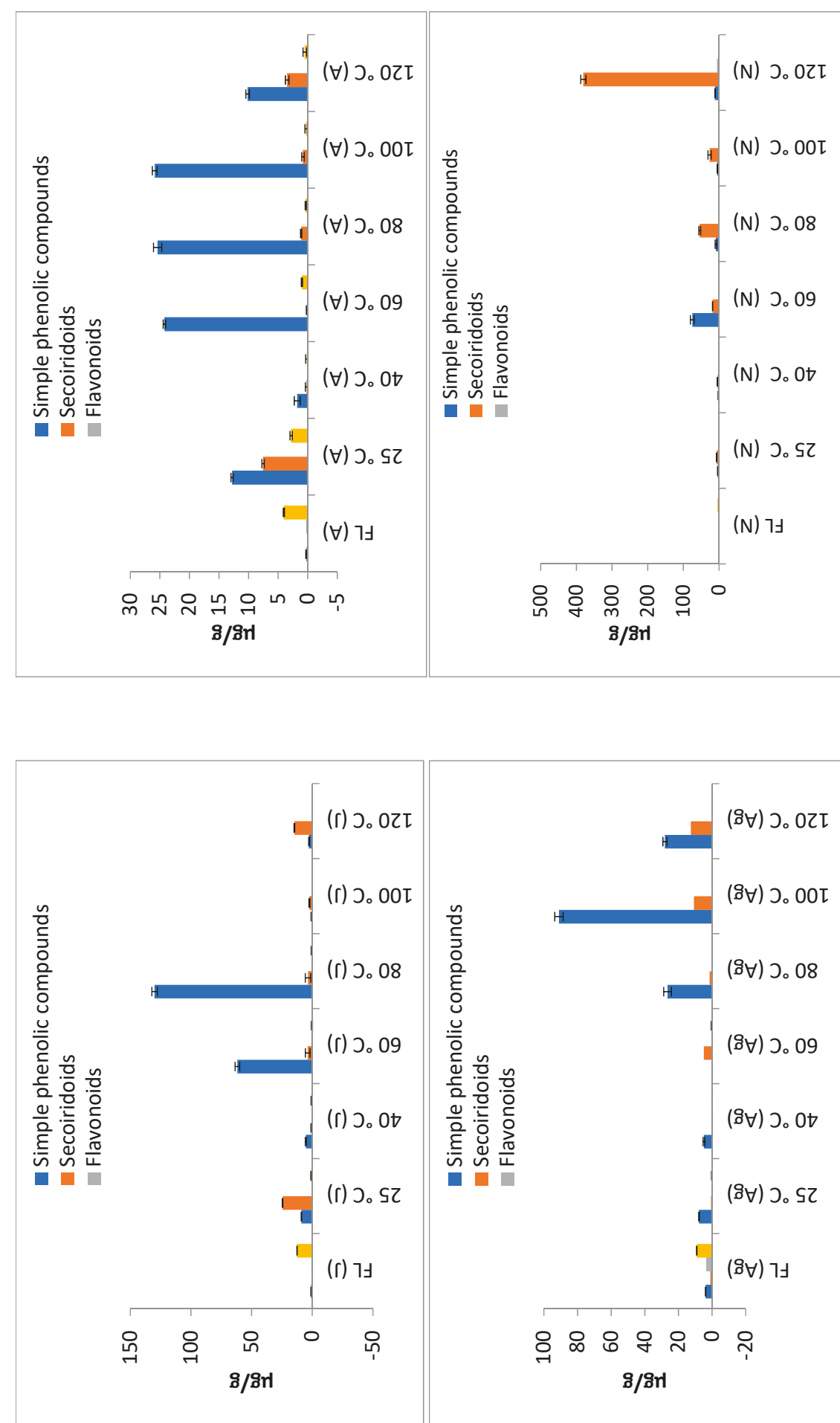


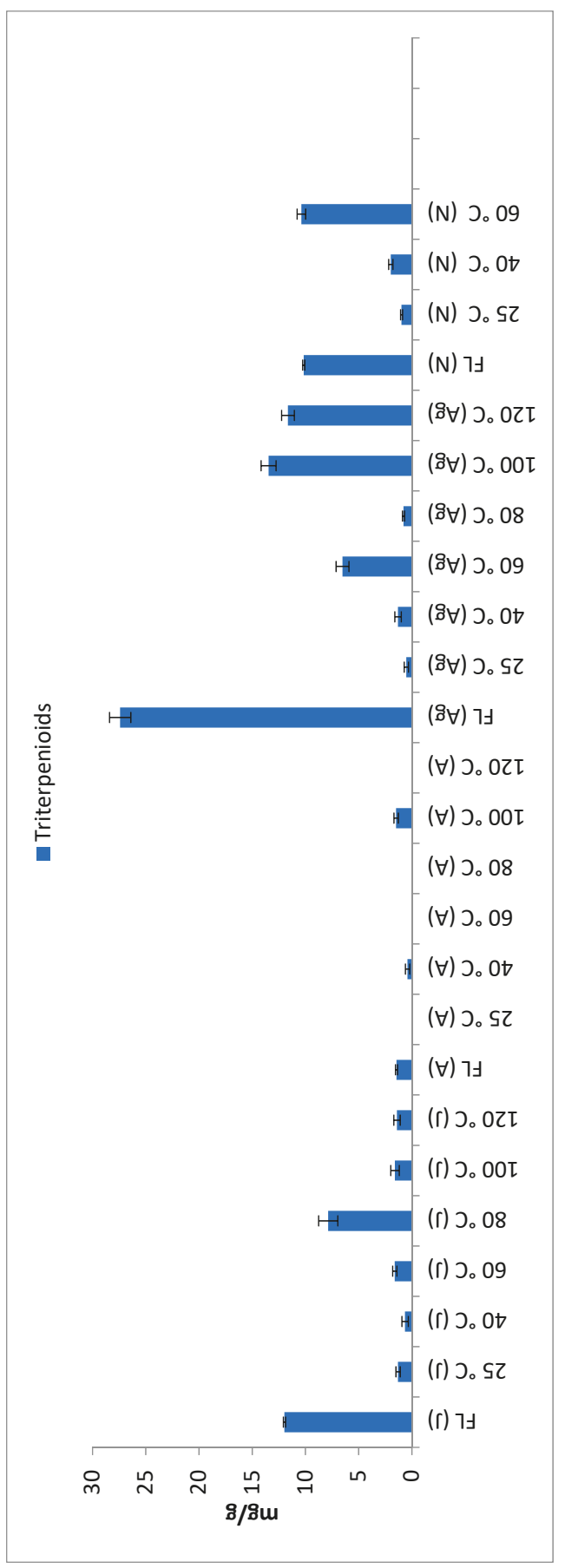

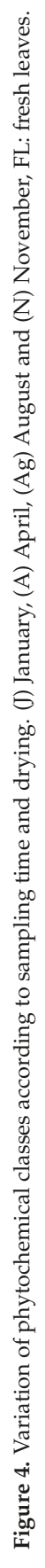


Total triterpenoids showed a significant variation $(p<0.05)$ between olive leaf samples according to sampling time. These metabolites were almost absent in April (Figure 3). The highest value of total triterpenoids was registered for extracts of the November and August sampling times, in which it reached 18.8 and $27.4 \mathrm{mg} / \mathrm{g}$, respectively. The amounts in January varied between 0.1 and $11.9 \mathrm{mg} / \mathrm{g}$. Regardless of the season, generally, ursolic and oleanolic acid presented the major fraction of triterpenoids, whereas maslinic acid presented the lowest amounts.

\section{Effect of Drying}

The contents of phenolic compounds in the leaves showed different fluctuations according to the drying temperature.

Secoiridoids showed a significant variation $(p<0.05)$ among samples from the same season and dried at different temperatures. Values varied from 0.6 to $24.5 \mu \mathrm{g} / \mathrm{g}, 0.1$ to $7.5 \mu \mathrm{g} / \mathrm{g}, 0.6$ to $4.9 \mu \mathrm{g} / \mathrm{g}$, and 4.1 to $380.0 \mu \mathrm{g} / \mathrm{g}$ for olive leaf samples collected in January, April, August, and November, respectively. The highest amounts were registered for leaves dried at $120{ }^{\circ} \mathrm{C}$ followed by those dried at $80{ }^{\circ} \mathrm{C}$ collected in autumn, with values reaching 380 and $53 \mu \mathrm{g} / \mathrm{g}$, respectively.

Oleuropein contents varied between 0.5 and $23.2 \mu \mathrm{g} / \mathrm{g}, 0.1$ and $6.9 \mu \mathrm{g} / \mathrm{g}, 0.6$ and $2.1 \mu \mathrm{g} / \mathrm{g}$, and between 4.1 and $290.0 \mu \mathrm{g} / \mathrm{g}$ for olive leaf samples of January, April, August, and November, respectively. As can be seen, the highest value was registered at a drying temperature of $120{ }^{\circ} \mathrm{C}$ for the samples collected in November. For the two collecting times of January and April, it seems that 25 and $120{ }^{\circ} \mathrm{C}$ were the appropriate drying temperatures to obtain the highest secoiridoid content, while 60 and $120^{\circ} \mathrm{C}$ leaf drying provided the highest amounts of secoiridoids in August. Finally, when collected in November, leaves dried at 80 and $120^{\circ} \mathrm{C}$ gave the highest amounts of secoiridoids. Accordingly, we can consider a temperature of $120^{\circ} \mathrm{C}$ as convenient to obtain higher secoiridoid contents in olive leaves.

Oleuropein content of fresh olive leaves was very low as compared to its content in dried leaves, which might be due to the cell structure destruction of drying that allows any solvent to penetrate more easily. In a previous study, it was claimed that the low area of the surface facilitates the penetration of solvents into cells [5]. On the other side, it seems that supercritical fluid extraction does not permit the extraction of oleuropein when fresh leaves are used. This is in agreement with the results reported in our previous work [9]. Depending on the collecting season, the appropriate drying temperature for obtaining the highest amounts of oleuropein was as follows $-25{ }^{\circ} \mathrm{C}$ when collected in January and April, and $120{ }^{\circ} \mathrm{C}$ when collected in August and November, reaching $290 \mu \mathrm{g} / \mathrm{g}$ in the latter. Previous studies reported that the composition of olive leaf extracts is greatly influenced by the drying technique [3,7]. In other work, oleuropein content of fresh green olive leaves was very low as compared to its content in dried leaves, explained by the fact that the surface area was too low to facilitate the penetration of solvents into cells so that oleuropein stayed protected in leaves cell [5].

In the same context, secologanoside contents showed remarkable fluctuations among olive leaf samples dried at different temperatures, with the leaves dried at $120{ }^{\circ} \mathrm{C}$ being those that gave the highest contents in secologanoside among all samples. Its value reached $32.4 \mu \mathrm{g} / \mathrm{g}$.

Elenolic acid glucoside was not detected in fresh olive leaves regardless of the sampling time. The most remarkable variation is registered for the sample dried at $120^{\circ} \mathrm{C}$ and collected in November in which its amount reached $12.8 \mu \mathrm{g} / \mathrm{g}$.

The major compound in lignans that was determined in the majority of the analyzed extracts was acetoxypinoresinol, of which amounts presented a mean value of more than $77 \%$ of determined lignans, followed by pinoresinol and syringaresinol. The highest amounts of lignans were found in fresh leaves independently of the sampling time and reached $12.6 \mu \mathrm{g} / \mathrm{g}$. Their levels showed a remarkable decrease with the increase in drying temperature.

Flavonoids amounts tended to decrease when increasing the drying temperature independently of the sampling time. For samples collected in November, when increasing drying temperature, flavonoids decreased gradually but increased then at $120^{\circ} \mathrm{C}$. The registered values of total flavonoids 
ranged from 0.2 to $0.8,0.1$ to 0.3 , and 0.2 to $5.3 \mu \mathrm{g} / \mathrm{g}$ for samples collected in January, April, and November, respectively.

Triterpenoids showed a significant variation $(p<0.05)$ among fresh and dried olive leaf samples at different temperatures, with the highest values registered in fresh leaves of the August sampling. The contents of determined triterpenoids varied from 0.2 to $11.9 \mathrm{mg} / \mathrm{g}$ in January, between 0.2 and $0.4 \mathrm{mg} / \mathrm{g}$ in April, 0.3 and $27.4 \mathrm{mg} / \mathrm{g}$ in August, and finally from 0.7 to $18.8 \mathrm{mg} / \mathrm{g}$ in November.

\subsection{Principal Component Analysis}

The principal component analysis permits us to better visualize the classification of the olive leaf samples under study according to the sampling time. Thus, PCA was applied to the data of the samples under study on the basis of classes of the identified phytochemicals.

As shown in Figure 5, for fresh leaves, the first two principal components (F1 and F2) explained $97.74 \%, 96.36 \%, 96.57 \%, 92.32 \%, 97.32 \%, 89.55 \%$ and $88.40 \%$ of the variance for fresh leaves, and leaves dried at $25,40,60,80,100$, and $120^{\circ} \mathrm{C}$, respectively. For fresh leaves, a positive correlation was observed between the axis $\mathrm{F} 1$ and secoiridoids, triterpenoids, flavonoids, and simple phenolic compounds $\left(\cos ^{2}>0.9\right)$ whereas lignans showed a positive correlation with the F2 axis. Good separation was observed between samples collected in August and the rest of the samples according to the F1 axis. Regarding the leaves dried at $25^{\circ} \mathrm{C}$, a positive correlation was registered between the $\mathrm{F} 1$ axis and secoiridoids, flavonoids, and other polar compounds ( $\cos ^{2}>0.85$ ), whereas a positive correlation was observed between the F2 axis and lignans and simple phenolic compounds $\left(\cos ^{2}>0.89\right)$. A clear separation was observed between the three main groups of samples (samples collected in January, samples collected in April, and samples collected in August and November). At a drying temperature of $40^{\circ} \mathrm{C}$, the PCAin Figure 5 showed a clear separation between samples collected in November and the rest of the samples. A positive correlation was registered between the F1 axis and secoiridoids, lignans, and flavonoids ( $\left.\cos ^{2}>0.95\right)$. At $60^{\circ} \mathrm{C}$, secoiridoids, other polar compounds and lignans which correlated highly to the F1 $\left(\cos ^{2}>0.81\right)$ axis permitted the separation between samples collected in November and the rest of samples. Three main groups of samples could be classified in the PCA applied at the data registered at $80^{\circ} \mathrm{C}$. Indeed, a clear separation could be visualized between samples collected in January, samples collected in November, and samples collected in April and August. When dried at $100{ }^{\circ} \mathrm{C}$, lignans and triterpenoids permitted a clear separation between samples collected in April and samples collected in August, while other polar compounds correlated to the F1 axis $\left(\cos ^{2}=0.882\right)$ and contributed to the separation of samples collected in November from the rest of samples. At $120^{\circ} \mathrm{C}$, secoiridoids were the most contributing variable in the classification of samples according to the $\mathrm{F} 1$ axis $\left(\cos ^{2}=0.947\right)$ while simple phenolic compounds were the most contributing variable in the classification of samples according to the $\mathrm{F} 2$ axis $\left(\cos ^{2}=0.851\right)$. 
(I)

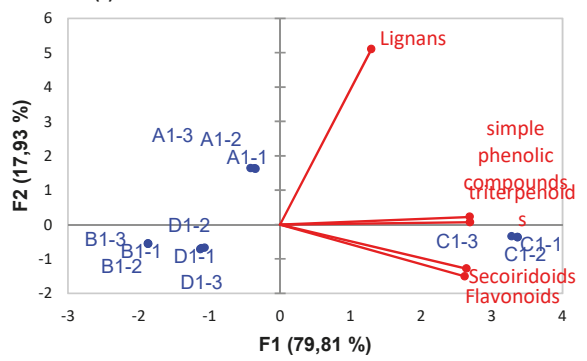

(III)

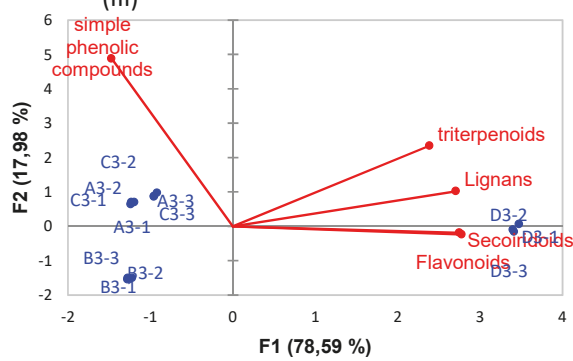

(V)

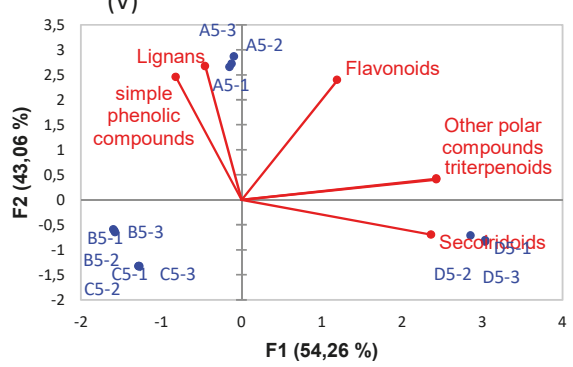

(VII)

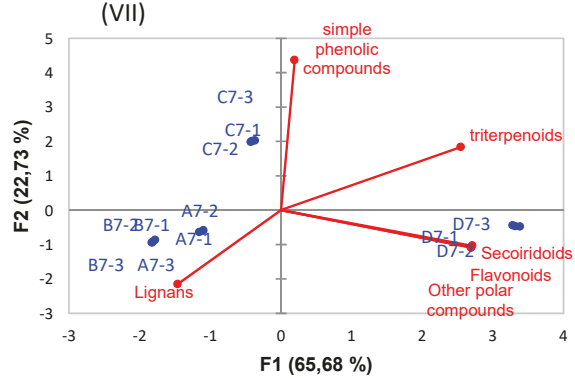

(II)
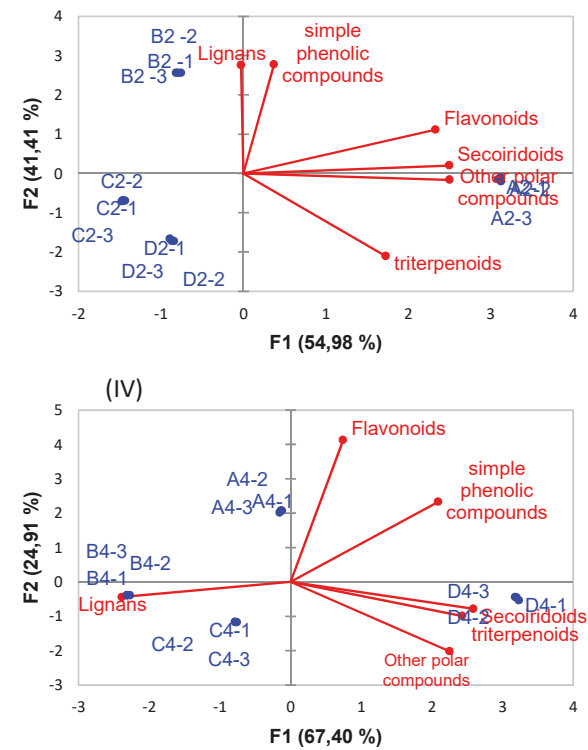

(VI)

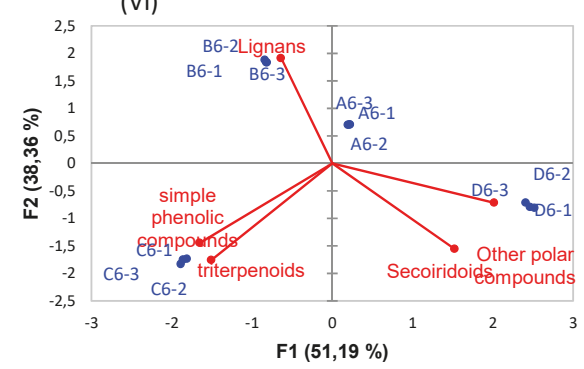

Figure 5. Principal Component Analysis of olive leaf samples according to sampling time: (I): Fresh leaves, (II): Leaves dried at $25^{\circ} \mathrm{C}$, (III): Leaves dried at $40{ }^{\circ} \mathrm{C}$, (IV): Leaves dried at $60^{\circ} \mathrm{C}$, (V): Leaves dried at $80^{\circ} \mathrm{C}$, (VI): Leaves dried at $100{ }^{\circ} \mathrm{C}$, and (VII): Leaves dried at $120^{\circ} \mathrm{C}$. A: January, B: April, C: August, and D: November, FL: fresh leaves.

In Figure 6, the plotting of olive leaf samples is presented according to drying temperatures. As can be observed, the most remarkable classification of samples was registered for the August and November samplings. In August, a clear separation was observed between fresh and dried leaves, regardless of the drying temperature. A high correlation was registered between the F1 axis and 
lignans and flavonoids $\left(\cos ^{2}>0.98\right)$. In November, a good separation was observed between the leaves dried at $120^{\circ} \mathrm{C}$ and the rest of the samples. Secoiridoids and other polar compounds correlated highly to the $\mathrm{F} 1$ axis $\left(\cos ^{2}>0.96\right)$.

For the majority of cases, flavonoids contents decreased with the increase of drying temperature except for November sampling time when the highest amount was registered at $120^{\circ} \mathrm{C}$.

(I)

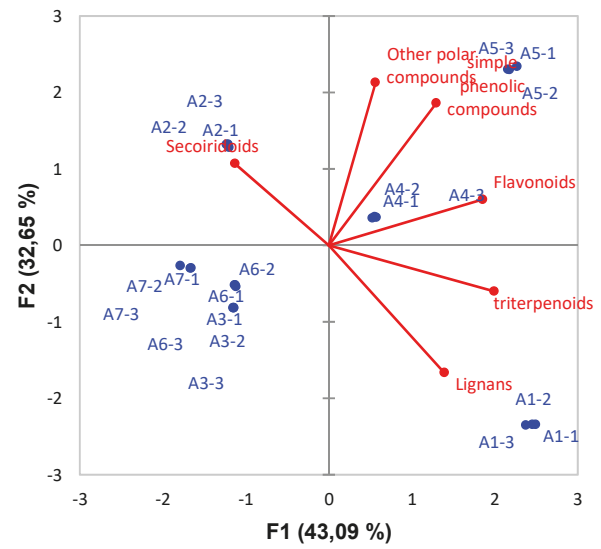

(III)

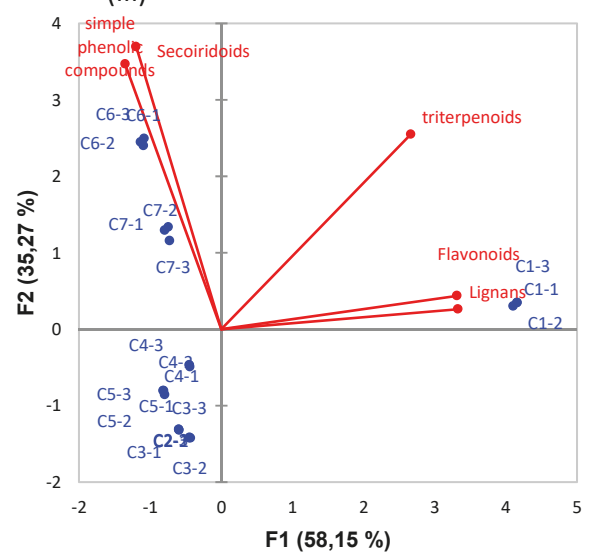

(II)

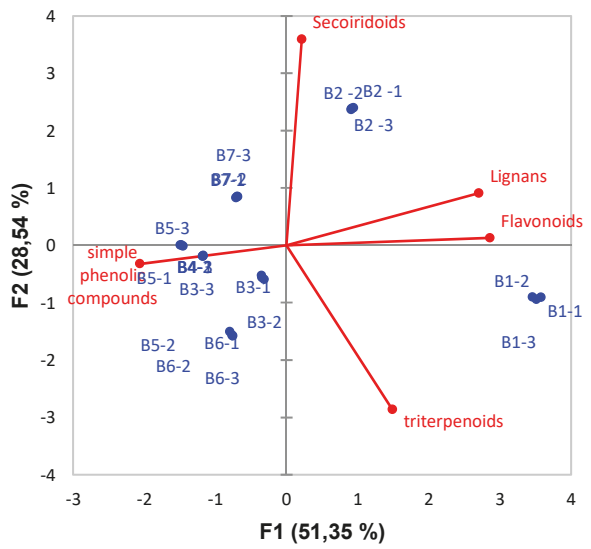

(IV)

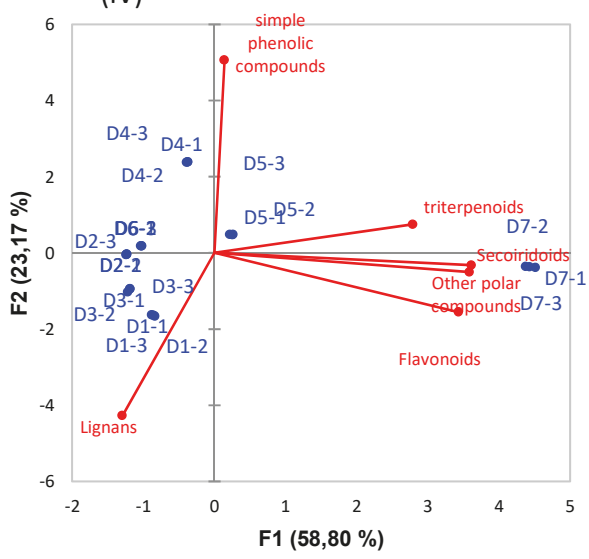

Figure 6. Principal Component Analysis of olive leaf samples according to drying temperature. (I) January, (II) April, (III) August, and (IV) November. 1: fresh leaves, 2: $25^{\circ} \mathrm{C}, 3: 40^{\circ} \mathrm{C}, 4: 60^{\circ} \mathrm{C}, 5$ : $80^{\circ} \mathrm{C}, 6: 100{ }^{\circ} \mathrm{C}$, and $7: 120^{\circ} \mathrm{C}$.

\section{Materials and Methods}

\subsection{Chemicals}

All chemicals were of analytical reagent grade and used as received. HPLC-grade acetonitrile and methanol were purchased from Labscan (Dublin, Ireland). Acetic acid of analytical grade (assay $>99.5 \%$ ) was purchased from Fluka (Buchs, Switzerland). Double-deionized water with a conductivity of $<18.2 \mathrm{M} \Omega$ was obtained with a Milli-Q system (Millipore, Bedford, MA). Standard compounds such as hydroxytyrosol, vanillin, luteolin, apigenin, ferulic acid, oleanolic, and maslinic acids were purchased from Sigma-Aldrich (St. Louis, MO, USA,) (+)-pinoresinol was acquired from Arbo Nova (Turku, Finland), and oleuropein from Extrasynthèse (Lyon, France). 


\subsection{Sampling and Drying}

Olive leaves were collected from the olive cultivar 'El Hor' from the Center of Tunisia. The collection of the leaf samples began from January 2017 and continued until November 2017. The collected olive leaves were directly transferred to the laboratory, washed with distilled water. Portions of leaves were immediately stored at $-80{ }^{\circ} \mathrm{C}$ and other portions were dried either at room temperature $\left(25^{\circ} \mathrm{C}\right)$ in a programmable mechanical convection oven (Binder Gmbh) or various temperatures of 40,60, 80, 100, and $120^{\circ} \mathrm{C}$. Leaves were ground using an Ultra Centrifugal Mill ZM 200 (Retsch Gmbh, Germany). As a means of gaining representative results and eliminating factors which could affect the monitoring of the phenolic compounds, this fraction was isolated by supercritical $\mathrm{CO}_{2}$ extraction without storage of dried samples.

\subsection{Supercritical $\mathrm{CO}_{2}$ Extraction}

Supercritical $\mathrm{CO}_{2}$ extraction was carried out with a Waters Prep Supercritical Fluid Extraction system (SFE-100) equipped with a high-pressure $\mathrm{CO}_{2}$ P-50 pump, a high-pressure co-solvent P-50 pump, an automated back pressure regulator, a low-pressure heating exchange, a high-pressure heating exchange, a high-pressure extraction, and a high-pressure collection vessel. Prior to the extraction process, $10 \mathrm{~g}$ of ground olive leaves were homogenized with $15 \mathrm{~g}$ of sea sand, that was selected as inert material to hold the sample inside the extraction cell and to improve extraction efficiency. This mixture was introduced into the extraction cell and packed with glass wool. Extractions were carried out at 150 bar and $40{ }^{\circ} \mathrm{C}$, once experimental conditions had been reached, the extraction solvent (consisting of a mixture of $\mathrm{CO}_{2}$ plus $6.6 \%$ of ethanol as a modifier) passed through the extraction cell for one hour at $23 \mathrm{~g} / \mathrm{min}$. The obtained extract was collected, and the solvent was evaporated under vacuum at $38^{\circ} \mathrm{C}$ [9]. A minimum of three replicate extractions was performed for each plant sample, and each extract was analyzed at least three times by HPLC analysis.

\subsection{RP-HPLC-TOF MS Analysis of Phenolic and Terpenoid Compounds}

Analytical methods to characterize the phenolic and terpenoid compounds in olive leaf SFE extracts were performed in an Agilent 1200 series Rapid Resolution LC (Agilent Technologies, CA, USA) equipped with a vacuum degasser, autosampler, a binary pump, and a diode array detector (DAD). The HPLC system was coupled to a micrOTOF (BrukerDaltonics, Bremen, Germany), and an orthogonal-accelerated TOF mass spectrometer using an electrospray interface (model G1607A from Agilent Technologies, Palo Alto, CA, USA). The chromatographic separation of these compounds was carried out on a $\mathrm{C}_{18}$ Zorbax Eclipse Plus analytical column $(4.6 \times 150 \mathrm{~mm}, 1.8 \mu \mathrm{m})$ from Agilent Technologies.

Regarding phenolic compounds, the mobile phases used were water with acetic acid $(0.5 \%)$ (mobile phase A), and acetonitrile (mobile phase B) and the solvent gradient changed according to the following conditions (Table 2):

Table 2. Solvent gradient conditions for the analysis of phenolic compounds.

\begin{tabular}{|c|c|c|}
\hline Time & Water $+0.5 \%$ Acetic Acid & Acetonitrile \\
\hline 0-10 min & $95 \%-70 \%$ & $5 \%-30 \%$ \\
\hline $10-12 \mathrm{~min}$ & $70 \%-67 \%$ & $30 \%-33 \%$ \\
\hline 12-17 min & $67 \%-62 \%$ & $33 \%-38 \%$ \\
\hline $17-20 \mathrm{~min}$ & $62 \%-50 \%$ & $38 \%-50 \%$ \\
\hline $20-23 \mathrm{~min}$ & $50 \%-5 \%$ & $50 \%-95 \%$ \\
\hline $23-25 \mathrm{~min}$ & $5 \%-95 \%$ & $95 \%-5 \%$ \\
\hline $25-35 \mathrm{~min}$ & $95 \%$ & $5 \%$ \\
\hline
\end{tabular}

The injection volume in the HPLC was $10 \mu \mathrm{L}$. The flow rate used was set at $0.80 \mathrm{~mL} / \mathrm{min}$ throughout the gradient and, consequently, the use of a splitter was required for the coupling with 
the MS detector, as the flow that arrived at the TOF detector had to be $0.2 \mathrm{~mL} / \mathrm{min}$ in order to obtain reproducible results and stable spray. Source and transfer parameters for MS analysis were set using the negative ion mode with spectra acquired over a mass range from $m / z 50$ to 1000 [9]. External mass spectrometer calibration was performed with sodium acetate clusters $(5 \mathrm{mM}$ sodium hydroxide in water $/ 2$-propanol $1 / 1(v / v)$, with $0.2 \%$ of acetic acid) in quadratic high-precision calibration (HPC) regression mode. The calibration solution was injected at the beginning of the run, and all the spectra were calibrated prior to polyphenols identification.

Separation of triterpenic compounds from olive leaf extracts was performed on the same 1200 series Rapid Resolution LC (Agilent Technologies, CA, USA) described above. The mobile phases used were acidified water with $0.1 \%$ formic acid as mobile phase A and methanol with $0.1 \%$ formic acid as mobile phase B (Table 3). The gradient was as follows:

Table 3. Solvent gradient conditions for the analysis of triterpenoids.

\begin{tabular}{ccc}
\hline Time & Water $+\mathbf{0 . 1} \%$ Formic Acid & Methanol $+\mathbf{0 . 1} \%$ Formic Acid \\
\hline $\mathbf{5} \mathbf{~}$ in & $25 \%$ & $75 \%$ \\
$\mathbf{1 0} \mathbf{m i n}$ & $0 \%$ & $100 \%$ \\
$\mathbf{2 0} \mathbf{~ m i n}$ & $0 \%$ & $100 \%$ \\
$\mathbf{2 8} \mathbf{m i n}$ & $95 \%$ & $5 \%$ \\
$\mathbf{3 5} \mathbf{m i n}$ & $95 \%$ & $5 \%$ \\
\hline
\end{tabular}

The injection volume in the HPLC was $10 \mu \mathrm{L}$. The flow rate was at $0.8 \mathrm{~mL} / \mathrm{min}$. The same MS conditions as described above were used, using sodium formate clusters for external mass spectrometer calibration.

Quantitation was carried out by HPLC-ESI-TOF-MS. Nine standard calibration curves of the main compounds found in samples were prepared using nine commercial standards. The stock solutions containing these analytes were prepared in methanol/water $(50 / 50, v / v)$. All calibration curves showed good linearity over the range of study with a minimum value of $R^{2}=0.992$. Results are given in $\mathrm{mg}$ of analyte per $\mathrm{g}$ of olive leaf for triterpenoids and in $\mu \mathrm{g}$ of analyte per $\mathrm{g}$ of olive leaf for the rest of compounds.

\subsection{Statistical Analysis}

An analysis of variance (ANOVA) and a Tukey posthoc test were carried out using IBM SPSS statistics 2017 version 25. Principal component analysis (PCA) was carried out using XLSTAT version 2018. 5.03. Values are expressed as mean value \pm SD of three replicates for each sample.

\section{Conclusions}

In conclusion, for fresh leaves, November was the best time to obtain extracts richer in simple phenolic compounds, secoiridoids, flavonoids, and triterpenoids as compared to the other sampling times. However, after drying, the behavior of the phenolic compounds and triterpenoids varied according to the drying temperature. In fact, at $25^{\circ} \mathrm{C}$, January sampling demonstrated the highest contents of secoiridoids and flavonoids, whereas April showed the highest simple phenolic compounds amounts. On the other hand, when dried at $40^{\circ} \mathrm{C}$, leaves collected in November showed the highest amounts in secoiridoids, flavonoids, lignans, and triterpenoids, whereas leaves collected in January and August showed the highest amounts in simple phenolic compounds. The same was observed for secoiridoids and triterpenoids for leaves collected in November at drying temperatures of $60^{\circ} \mathrm{C}$ and higher. Accordingly, April seemed to be the best sampling time for better recovery of lignans, and August seemed to be the best time for recovering higher simple phenolic compounds at elevated drying temperature.

Among analyzed samples, fresh leaves were generally characterized by higher amounts of flavonoids, lignans, and triterpenoids, whereas secoiridoids were generally found at higher amounts 
in samples dried at $120^{\circ} \mathrm{C}$. It is noteworthy that samples dried at $120^{\circ} \mathrm{C}$ and collected in November presented among analyzed samples with the highest amounts in secoiridoids, flavonoids, triterpenoids, and other polar compounds. Since knowing the qualitative and quantitative composition as well as the occurrence of the phytochemicals in a given plant species is a crucial step for investigating the bioavailability and biochemical effect of any dietary phytochemical, and such results would be very useful in the study of the implication of olive leaf phytochemicals in biological activities. In addition, such findings would be helpful in terms of industrial valorization of El Hor olive leaves since it is important to know the best sampling time and drying temperature to obtain the highest yield of compounds of interest. Olive leaf bioactive compounds can be used as an important source to produce nutraceuticals or to be included in functional food thanks to their potential health benefits.

Supplementary Materials: The following are available online at http://www.mdpi.com/1996-1073/12/5/947/s1. Statistical data on phenolic and triterpenoid compounds determined according to the drying temperature and sampling season.

Author Contributions: Investigation, A.T., J.L.Z. and H.J.; Methodology, A.T. and J.L.Z.; Supervision, J.L.Z., L.A., A.S.C., D.A.R. and J.Y.C.; Validation, A.T. and; Writing-Original Draft, A.T., J.L.Z. and N.T.; Writing-Review \& Editing; L.A., A.S.C., J.Y.C. and D.A.R.

Acknowledgments: This work was supported by the Tunisian Ministry of Higher Education under the Tunisian-Korean project "Development of Anti-arthritic remedy with Olive by-products".

Conflicts of Interest: The authors declare no conflict of interest.

\section{Abbreviations}

$\begin{array}{ll}\text { HPLC } & \text { High-Performance Liquid Chromatography } \\ \text { ESI } & \text { Electrospray Ionization } \\ \text { TOF } & \text { Time of Flight } \\ \text { MS } & \text { Mass Spectrometry } \\ \text { BPC } & \text { Base Peak Chromatogram } \\ \text { EIC } & \text { Extracted Ion Chromatogram } \\ \text { SFE } & \text { Supercritical Fluid Extraction } \\ \text { FL } & \text { Fresh olive leaves }\end{array}$

\section{References}

1. Segasothy, M.; Phillips, P.A. Vegetarian diet: panacea for modern lifestyle diseases? QJM 1999, 92, 531-544. [CrossRef] [PubMed]

2. Nicoletti, M. Advances in Production of Functional Foods and Nutraceuticals; Nova Science Publishers, Inc.: New York, NY, USA, 2014; ISBN 9781631172557.

3. Ahmad-Qasem, M.H.; Cánovas, J.; Barrajón-Catalán, E.; Carreres, J.E.; Micol, V.; García-Pérez, J.V. Influence of Olive Leaf Processing on the Bioaccessibility of Bioactive Polyphenols. J. Agric. Food Chem. 2014, 62, 6190-6198. [CrossRef] [PubMed]

4. Fabbri, A.; Galaverna, G.; Ganino, T. Polyphenol composition of olive leaves with regard to cultivar, time of collection and shoot type. Acta Hortic. 2008, 791 Pt 2, 459-464. [CrossRef]

5. Afaneh, I.; Yateem, H.; Al-Rimawi, F. Effect of Olive Leaves Drying on the Content of Oleuropein. Am. J. Anal. Chem. 2015, 6, 246-252. [CrossRef]

6. Erbay, Z.; Icier, F. Thin-Layer Drying Behaviors of Olive Leaves (Olea europaea L.). J. Food Process Eng. 2010, 33, 287-308. [CrossRef]

7. Ahmad-Qasem, M.H.; Barrajón-Catalán, E.; Micol, V.; Mulet, A.; García-Pérez, J.V. Influence of freezing and dehydration of olive leaves (var. Serrana) on extract composition and antioxidant potential. Food Res. Int. 2013, 50, 189-196. [CrossRef]

8. Erbay, Z.; Icier, F. Optimization of Drying of Olive Leaves in a Pilot-Scale Heat Pump Dryer. Dry. Technol. 2009, 27, 416-427. [CrossRef] 
9. Taamalli, A.; Arráez-Román, D.; Barrajón-Catalán, E.; Ruiz-Torres, V.; Pérez-Sánchez, A.; Herrero, M.; Ibañez, E.; Micol, V.; Zarrouk, M.; Segura-Carretero, A.; et al. Use of advanced techniques for the extraction of phenolic compounds from Tunisian olive leaves: Phenolic composition and cytotoxicity against human breast cancer cells. Food Chem. Toxicol. 2012, 50, 1817-1825. [CrossRef] [PubMed]

10. Barrajón-Catalán, E.; Taamalli, A.; Quirantes-Piné, R.; Roldan-Segura, C.; Arráez-Román, D.; Segura-Carretero, A.; Micol, V.; Zarrouk, M. Differential metabolomic analysis of the potential antiproliferative mechanism of olive leaf extract on the JIMT-1 breast cancer cell line. J. Pharm. Biomed. Anal. 2015, 105, 156-162. [CrossRef] [PubMed]

11. Ksouri, W.M.; Medini, F.; Mkadmini, K.; Legault, J.; Magné, C.; Abdelly, C.; Ksouri, R. LC-ESI-TOF-MS identification of bioactive secondary metabolites involved in the antioxidant, anti-inflammatory and anticancer activities of the edible halophyte Zygophyllum album Desf. Food Chem. 2013, 139, 1073-1080. [CrossRef] [PubMed]

12. Segura-Carretero, A.; Menéndez-Menéndez, J.; Fernández-Gutiérrez, A. Polyphenols in Olive Oil: The Importance of Phenolic Compounds in the Chemical Composition of Olive Oil. In Olives and Olive Oil in Health and Disease Prevention; Elsevier Inc.: Amsterdam, The Netherlands, 2010; pp. 167-175, ISBN 9780123744203.

13. Taamalli, A.; Arráez-Román, D.; Ibañez, E.; Zarrouk, M.; Segura-Carretero, A.; Fernández-Gutiérrez, A. Optimization of microwave-assisted extraction for the characterization of olive leaf phenolic compounds by using HPLC-ESI-TOF-MS/IT-MS2. J. Agric. Food Chem. 2012, 60, 791-798.

14. Taamalli, A.; Abaza, L.; Arráez Román, D.; Segura Carretero, A.; Fernández Gutiérrez, A.; Zarrouk, M.; Ben Youssef, N. Characterisation of phenolic compounds by HPLC-TOF/IT/MS in buds and open flowers of "chemlali" olive cultivar. Phytochem. Anal. 2013, 24, 504-512. [CrossRef] [PubMed]

15. Abaza, L.; Taamalli, A.; Arráez-Román, D.; Segura-Carretero, A.; Fernández-Gutierrérez, A.; Zarrouk, M.; Youssef, N. Ben Changes in phenolic composition in olive tree parts according to development stage. Food Res. Int. 2017, 100, 454-461. [CrossRef] [PubMed]

16. Ammar, S.; del Mar Contreras, M.; Gargouri, B.; Segura-Carretero, A.; Bouaziz, M. RP-HPLC-DAD-ESI-QTOF-MS based metabolic profiling of the potential Olea europaea by-product "wood" and its comparison with leaf counterpart. Phytochem. Anal. 2017, 28, 217-229. [CrossRef] [PubMed]

17. Taamalli, A.; Arráez Román, D.; Gómez Caravaca, A.M.; Zarrouk, M.; Segura Carretero, A. Geographical Characterization of Tunisian Olive Tree Leaves (cv. Chemlali) Using HPLC-ESI-TOF and IT/MS Fingerprinting with Hierarchical Cluster Analysis. J. Anal. Methods Chem. 2018, 2018, 6789704. [CrossRef] [PubMed]

18. Taamalli, A.; Arráez-Román, D.; Abaza, L.; Iswaldi, I.; Fernández-Gutiérrez, A.; Zarrouk, M.; Segura-Carretero, A. LC-MS-based Metabolite Profiling of Methanolic Extracts from the Medicinal and Aromatic Species Mentha pulegium and Origanum majorana. Phytochem. Anal. 2015, 26, 320-330. [CrossRef] [PubMed]

19. Rodríguez-Pérez, C.; Quirantes-Piné, R.; Fernández-Gutiérrez, A.; Segura-Carretero, A.; Rodriguez-Perez, C.; Quirantes-Pine, R.; Fernandez-Gutierrez, A. Comparative characterization of phenolic and other polar compounds in Spanish melon cultivars by using high-performance liquid chromatography coupled to electrospray ionization quadrupole-time of flight mass spectrometry. Food Res. Int. 2013, 54, 1519-1527. [CrossRef]

20. Kalogeropoulos, N.; Chiou, A.; Ioannou, M.; Karathanos, V.T.; Hassapidou, M.; Andrikopoulos, N.K. Nutritional evaluation and bioactive microconstituents (phytosterols, tocopherols, polyphenols, triterpenic acids) in cooked dry legumes usually consumed in the Mediterranean countries. Food Chem. 2010, 121, 682-690. [CrossRef]

21. Caligiani, A.; Malavasi, G.; Palla, G.; Marseglia, A.; Tognolini, M.; Bruni, R. A simple GC-MS method for the screening of betulinic, corosolic, maslinic, oleanolic and ursolic acid contents in commercial botanicals used as food supplement ingredients. Food Chem. 2013, 136, 735-741. [CrossRef] [PubMed]

22. Ranalli, A.; Contento, S.; Lucera, L.; Di Febo, M.; Marchegiani, D.; Di Fonzo, V. Factors affecting the contents of iridoid oleuropein in olive leaves (Olea europaea L.). J. Agric. Food Chem. 2006, 54, 434-440. [CrossRef] [PubMed]

(C) 2019 by the authors. Licensee MDPI, Basel, Switzerland. This article is an open access article distributed under the terms and conditions of the Creative Commons Attribution (CC BY) license (http:/ / creativecommons.org/licenses/by/4.0/). 

Optimization of Oleuropein and Luteolin-7-O-Glucoside Extraction from Olive Leaves by Ultrasound-Assisted Technology

\author{
Antonio Lama-Muñoz *, María del Mar Contreras, Francisco Espínola, Manuel Moya, \\ Inmaculada Romero and Eulogio Castro \\ Department of Chemical, Environmental and Materials Engineering, University of Jaén, Campus Las Lagunillas, \\ s/n, Building B3, 23071 Jaén, Spain \\ * Correspondence: alama@ujaen.es; Tel.: +34-953-211-860
}

Received: 29 May 2019; Accepted: 27 June 2019; Published: 28 June 2019

\begin{abstract}
The olive orchard cultivation in Mediterranean countries results in huge amounts of lignocellulosic biomass residues. One of the main residues are olive leaves. Olive leaves contain high concentrations of bioactive antioxidant compounds like oleuropein and luteolin-7-glucoside. The production of biactive compounds from olive leaves requires treatments capable of breaking the lignocellulosic structure. Current research focuses on use of inexpensive, quick, and not harmful to the environment treatments, searching a more simplified large-scale operation approach. Recently, advances in applied chemistry have led to possible new emerging industrial techniques like ultrasound-assisted extraction (UAE). This technology is a promising candidate as a green treatment solution for olive leaves utilization in a biorefinery. However, this application goes through prior optimization of technique and operating conditions. The goal of this study was to optimize the extraction of oleuropein and luteolin-7-glucoside from olive leaves through an investigation of the influence of key factors of ultrasound-assisted extraction using an experimental central composite design, in comparison with conventional Soxhlet extraction. The highest extraction efficiency and antioxidant capacity were obtained under optimal increment of temperature and amplitude conditions ( $40{ }^{\circ} \mathrm{C}$ and $30 \%$, respectively). Values for oleuropein, luteolin-7-glucoside were $69.91 \mathrm{~g} / \mathrm{kg}$ and $1.82 \mathrm{~g} / \mathrm{kg}$, respectively.
\end{abstract}

Keywords: antioxidants; bioactive compounds; biorefinery; experimental design; olive leaves; olive biomass; response surface methodology; ultrasound-assisted extraction; olive byproducts valorization

\title{
1. Introduction
}

The activity of the olive oil industry generates enormous quantities of solid wastes; among them olive leaves represent $10 \%$ of the total weight of the olives harvested. They are also generated in high amounts during olive tree pruning ( $25 \mathrm{~kg}$ per olive tree annually) [1]. Disposal of olive leaves help to maintain fields clean and avoid the spread of diseases; they are usually removed by either burning or grinding and scattering them on fields, which involves a very important problem of management and environmental pollution [2]. Nowadays there are no exploitations on an industrial scale for this byproduct, so proposals for evaluation and exploitation can help its better use and valorization.

Olive leaves represent a promising raw source of antioxidants and bioactive substances. Olive leaves contain high amounts of a large variety of phenolic and flavonoid compounds (hydroxytyrosol, verbascoside, apigenin-7-glucoside, rutin, and ligstroside, among others), similar to those present in olives and their derived products [3,4]. Because of that, there is a great interest by the scientific community in the valorization of these residues to achieve extracts enriched in these compounds that can be used in food, cosmetic, and pharmaceutical industries $[5,6]$ to prevent oxidation processes. 
In addition, olive leaves extracts have other applications in food industry such as to improve the nutritive value of certain foods [7]. Among the bioactive compounds of olive leaves are secoiridoids and flavonoids, oleuropein and the flavone luteolin-7-glucoside being some of the compounds that have attracted more interest and which show well-documented biologic activities including antioxidant, antimicrobial, anti-inflammatory, anticancer, and neuroprotective properties [8]. Oleuropein and luteolin-7-glucoside are the most important phenolic compounds recovered from olive leaves [9]. Oleuropein is the most abundant component of the secoiridoid fraction, whereas glycosylated form luteolin-7-glucoside is the principal flavonoid with concentrations can reach up to 210 and $11 \mathrm{~g} / \mathrm{kg}$ dry leaf, respectively $[10,11]$.

Literature shows that oleuropein has potent biological and pharmacological activities [12]. Its main pharmacological properties that make it anticancer, anti-diabetes, antiobesity, cardioprotective, gastroprotective, hepato-protective, and radioprotective, among others, are in large part attributed to its putative antioxidant and anti-inflammatory effects [13]. Oleuropein and luteolin-7-glucoside have also been studied for their differentiation-inducing effects on hematopoietic stem cells highlighting their potential use in the ex vivo generation of blood cells components that are very important for the therapy of a wide spectrum of hematological disorders [14]. In a work carried out by Ahmad-Qasem et al. [15] on the olive leaves extracts' bioaccessibility, luteolin-7-glucoside was the most stable polyphenol during the in vitro digestion process simulation which means this compound may be considered as one of the responsible of the bioactivity of olive leaves extracts. Therefore, owing to their numerous health benefits associated, an optimized extraction method is important for their isolation and purification, thus contributing to the advances in research of their biological properties.

Several extraction technologies have been used to obtain antioxidant extracts and to isolate bioactive compounds from olive leaves [16]. The optimization of the extraction techniques for these compounds is essential for the development of biorefineries dedicated to the valorization of the by-products generated by olive biomass for obtaining pharmaceuticals, cosmetics, nutraceuticals, and functional foods. The conventional or traditional methods such as Soxhlet solid-liquid extraction have many drawbacks, such as long extraction times and exposure of the extracts to excessive heat. Recently, the demand of novel techniques of extraction with shorter extraction times and less consumption of solvent than conventional methods is increasing. As an alternative of traditional extraction methods, newer and more sophisticated technologies such as pressurized liquid extraction, also known as accelerated solvent extraction, microwave-assisted extraction, plasma-assisted extraction, supercritical fluid extraction, and ultrasound-assisted extraction (UAE) have been developed and used to obtain different types of bioproducts from various biomass [17]. For instance, pressurized liquid extraction has shown to be effective for the recovery of olive leaves biophenols [18]; microwave treatment has been utilized for the extraction of lipids from lyophilized algal biomass [19]; plasma-based processes have been used as pretreatment for the isolation of biopolymers from crustacean wastes [20], and supercritical carbon dioxide extraction is suited for the obtaining of hop extracts mostly constituted by non-polar compounds [21]. Specifically, the UAE process is an effective extraction technology for a broad range of compounds from different samples of biomass [22] and is being specifically used in the extraction of phenolic antioxidant and bioactive compounds from olive leaves [23-26] and other olive oil industry by-products $[27,28]$. UAE provides many advantages for natural compounds extraction since ultrasound waves produce intense pressure and temperature gradients within the material, which can induce physical structural disruption of cell wall due to the formation of microcavities. This then enhances the extraction rates by increasing the mass transfer and release of intracellular components into the extraction solvent, leading to higher extraction yields with shorter processing time [23]. The application of UAE in food industry is an interesting alternative to the traditional extraction methods. It is considered one of the most economically and feasible large-scale applications [29].

UAE is influenced by several factors such as temperature and amplitude (maximum height of a soundwave). Therefore, the optimization of the extraction procedure is critical to produce extracts with high content of oleuropein and luteolin-7-O-glcuoside. Experimental designs are useful tools 
for evaluation of statistical significance of the factors implicated and the simultaneous optimization of variables. In comparison to one-variable-at-a-time protocol, the experimental design reduces the experimental trials, analysis time, and the environmental and economic cost and error [30]. Experimental design under response surface methodology (RSM) is a useful mathematical and statistical protocol to assess the influence of multiple factors and their interactions on one or more response variables. RSM has been used by many authors for optimization of bioactive compounds extraction from a number of biomass sources, including olive leaves, using UAE [31].

Although the effect of extraction conditions on the oleuropein content has been investigated, no study reports on the effects of UAE extraction factors on the recovery and simultaneous optimization of the other main polyphenol from the olive leaves extracts, the compound luteolin-7-glucoside. Optimization of UAE has been studied by extraction parameters including solid/solvent ratio, time, and ethanol concentration. However, most of the authors did not include temperature and/or amplitude as design factors [32-34]. Thus, the goal of this study was to optimize the extraction of oleuropein and luteolin-7-glucoside from olive leaves through a study of the influence of two key factors of UAE such as increment of temperature and amplitude using a central composite design (CCD). Additionally, antioxidant capacity of the extracts as well as the total phenolic content (TPC) and total flavonoid content (TFC) were also included. Furthermore, these findings were compared to the traditional Soxhlet extraction method.

\section{Materials and Methods}

\subsection{Samples and Reagents}

Olive tree leaves from Picual variety were manually picked from specimens situated on the campus Las Lagunillas of the University of Jaén, Spain. After collection, raw material was dried in an oven for $24 \mathrm{~h}$ at $105{ }^{\circ} \mathrm{C}$ and ground using a cutting mill featuring a $5 \mathrm{~mm}$ sieve. The average particle diameter by mass was further evaluated $\left(D_{50}=1.21 \mathrm{~mm}\right)$. Finally, they were stored at room temperature in the dark until extraction. For determining the moisture content, a sample of fresh olive leaves was weighed, heated in an oven for $24 \mathrm{~h}$ at $105^{\circ} \mathrm{C}$, cooled in a desiccator, and then reweighed to constant weight in accordance with NREL analytical procedure (Technical Report NREL/TP-510-42621). Total solids and moisture content in fresh olive leaves expressed on weight basis were $51.44 \%(\mathrm{w} / \mathrm{w})$ and $48.56 \%(\mathrm{w} / \mathrm{w})$, respectively.

Acetonitrile, ethanol, and methanol (HPLC grade) were supplied by PanReac AppliChem (Barcelona, Spain). Commercial standards of oleuropein and luteolin-7-glucoside were provided by Sigma-Aldrich (St. Louis, USA).

\subsection{Extraction Methods}

Two methods were evaluated for the extraction of oleuropein and luteolin-7-glucoside from olive leaves.

Soxhlet extraction was carried out with $10 \mathrm{~g}$ of dry olive leaves and $150 \mathrm{~mL}$ of $60 \%(\mathrm{v} / \mathrm{v})$ ethanol-water solution for $4 \mathrm{~h}$. Literature shows that the aqueous ethanol solutions are required to effectively extract phenolic compounds from olive leaves [26,32,35]. Since aqueous ethanol solutions are the most common safe solvents in the food industrial process, they were selected as extraction solvent. The extractions were performed in triplicate. The extracts were filtered at the end of the experiments using grade 1 Whatman ${ }^{\circledR}$ qualitative filter paper. The results were expressed in mean \pm standard deviation.

Ultrasound-assisted extraction (UAE) was performed using a sonifier ultrasonic disrupter/ homogenizer 150 (Branson Ultrasonics Corporation, Danbury, USA) operating at a frequency of $40 \mathrm{kHz}$, equipped with a microtip ultrasound probe, and a temperature probe. Specifically, $2.0 \mathrm{~g}$ ground olive leaves were thoroughly mixed with $26 \mathrm{~mL}$ of $60 \%$ (v/v) ethanol-water solution (solvent/solid ratio of $13: 1 \mathrm{v} / \mathrm{w}$ ) in a glass beaker. The extraction was performed with the ultrasonic probe immersed 
$1 \mathrm{~cm}$ into the mixture. Before applying ultrasonics, all samples were put into a thermostatic bath at $20^{\circ} \mathrm{C}$ and maintained there until this temperature was reached. When an ultrasound pulse travels through sample, some of the energy is absorbed by the sample and is converted to heat, which in turn produces a temperature rise. The heating rate depends on the amplitude. Amplitude is a parameter used to describe the strength of an ultrasound pulse. Amplitude is a measure of a wave's magnitude of oscillation, that is, the magnitude of the maximum disturbance in the medium. Since sample heating occurs due to absorption of ultrasound energy by the solution and to self-heating of the probe, the extraction procedure was performed in maximum temperature mode and continuous sonics (continuous sonics were run at a set constant amplitude, until a set maximum temperature was measured by the temperature probe, time the assay was terminated). The increment of temperature was used as a design factor and the ultrasonics time was considered as a response to be studied. The effects of these UAE parameters were evaluated on the oleuropein and luteolin-7-glucoside content from olive leaves extracts. An ethanol-water $(60 \%, v / v)$ solution was used as extraction solvent. This solvent is commonly used for extracting under optimal conditions phenolic compounds from olive leaves [25] because it is a non-harmful, low-toxicity, and easily removable solvent that presents minimum environmental impact and was a good option for the UAE process.

\subsection{Design of Experiments and Statistical Analysis}

RSM was applied to the investigation of the effects of the factors increment of temperature and amplitude on the extraction of phenolic and flavonoid compounds from olive leaves. As the equipment cannot operate at more than $70 \%$ the maximum amplitude when using a microtip, in this design a range between $30 \%$ and $60 \%$ of maximum amplitude was selected. Seven responses were selected: the extraction yield or residual dry matter (R), total flavonoid and phenolic contents (TFC and TPC, respectively), antioxidant capacity (2,2-diphenyl-1-picrylhydroazyl (DPPH) free-radical scavenging capacity), oleuropein and luteolin-7-glucoside contents of the extracts, and applied ultrasonic time to reach the maximum temperature. To determine the relationships between the factors and observed results and to optimize the operating conditions, a design of experiments was performed: a central composite design (CCD) involving two factors, consisting of thirteen randomized trials, and including five replicates of the central point. The coded and actual factors for the experimental design are listed in Table 1, together with the maximum temperature reached by the sample. The factors were coded in accordance with the transformation reported by Vidal et al. [36].

As temperature is not an independent factor of the amplitude, the increment of temperature from $20^{\circ} \mathrm{C}$ was fixed as independent variable in experimental design. As mentioned in Section 2.2, prior to the extraction, all samples were tempered at $20^{\circ} \mathrm{C}$. The sonifier allowed us to control the way in which ultrasonics were applied to the samples by setting the unit to operate in one of the several different modes. Maximum temperature control mode was determined and specified operating temperatures for each ultrasonic cycle. Several maxima allowable temperatures in the samples or liquids were set for the experimental design $\left(40,50\right.$ and $\left.60^{\circ} \mathrm{C}\right)$, so that ultrasonics stopped automatically when the specified temperatures were reached and measured by the temperature probe.

The experimental data were evaluated using the Design-Expert $₫ v 8$ 8.0.7.1 software (Stat-Ease, Inc., USA) and response surface methodology. ANOVA test was used to analyze the significance of the data. To explain the behavior of the responses, a quadratic model for each response was used in accordance with Equation (1):

$$
Y=\beta_{0}+\beta_{1} \cdot \Delta \mathrm{T}+\beta_{2} \cdot \mathrm{A}+\beta_{12} \cdot \Delta \mathrm{T} \cdot \mathrm{A}+\beta_{11} \cdot \Delta \mathrm{T}^{2}+\beta_{22} \cdot \mathrm{A}^{2}+\mathrm{SD}
$$

where $\mathrm{Y}$ is the response, $\Delta \mathrm{T}$ is the coded increment of temperature $\left({ }^{\circ} \mathrm{C}\right), \mathrm{A}$ is the coded amplitude, and SD is the standard deviation of the models. The predicted responses were correlated with the following set of coefficients: the intercept $\left(\beta_{0}\right)$, linear $\left(\beta_{1}\right.$ and $\left.\beta_{2}\right)$, interaction $\left(\beta_{12}\right)$, and quadratic $\left(\beta_{11}\right.$ and $\beta_{22}$ ) coefficients. A significance level of $0.1 \%$ was set up for the models (p-value $<0.001$ ) and a $5 \%$ for the coefficients ( $p$-value $<0.05$ ). The lack of fit was statistically significant at the $10 \%$ probability 
level ( $p$-value $>0.1$ ). The terms not significant of the models were not incorporated to the equations in accordance with the $\mathrm{p}$-values indicated above.

Table 1. Central composite design (CCD) expressed in terms of actual and coded factors used for optimization of ultrasound-assisted extraction (UAE) conditions and maxima operating temperatures.

\begin{tabular}{|c|c|c|c|c|c|c|}
\hline \multirow{2}{*}{ Run } & \multirow{2}{*}{ Space Type } & \multicolumn{2}{|c|}{ Increment of Temperature $(\Delta \mathrm{T})$} & \multirow{2}{*}{$\begin{array}{c}\text { Maximum } \\
\text { Temperature }\left({ }^{\circ} \mathrm{C}\right)\end{array}$} & \multicolumn{2}{|c|}{ Amplitude (A) } \\
\hline & & Actual $\left({ }^{\circ} \mathrm{C}\right)$ & Coded & & Actual (\%) ${ }^{1}$ & Coded \\
\hline 1 & Center & 30 & 0 & 50 & 45 & 0 \\
\hline 2 & Axial & 30 & 0 & 50 & 23.8 & -1.413 \\
\hline 3 & Factorial & 20 & -1 & 40 & 60 & 1 \\
\hline 4 & Axial & 30 & 0 & 50 & 66.2 & 1.413 \\
\hline 5 & Factorial & 40 & 1 & 60 & 30 & -1 \\
\hline 6 & Axial & 15.9 & -1.410 & 35.9 & 45 & 0 \\
\hline 7 & Center & 30 & 0 & 50 & 45 & 0 \\
\hline 8 & Center & 30 & 0 & 50 & 45 & 0 \\
\hline 9 & Factorial & 20 & -1 & 40 & 30 & -1 \\
\hline 10 & Axial & 44.1 & 1.410 & 64.1 & 45 & 0 \\
\hline 11 & Factorial & 40 & 1 & 60 & 60 & 1 \\
\hline 12 & Center & 30 & 0 & 50 & 45 & 0 \\
\hline 13 & Center & 30 & 0 & 50 & 45 & 0 \\
\hline
\end{tabular}

\subsection{Extraction Yield}

To obtain the extraction yield, the extracts were filtered through a $0.45 \mu \mathrm{m}$ and an amount of $2 \mathrm{~mL}$ was dried using an electronic moisture analyzer model BMO35 (BOECO, Germany) at $105^{\circ} \mathrm{C}$ to constant weight. The extraction yields are referred to initial dry biomass and expressed as grams of extract per kilogram of dry olive leaf.

\subsection{TPC and TFC of Olive Leaves Extracts}

For the TPC quantification of the olive leaves extracts the Folin-Ciocalteu method was used. Briefly, $1.5 \mathrm{~mL}$ of $0.2 \mathrm{M}$ Folin's reagent and $1.2 \mathrm{~mL}$ of $0.7 \mathrm{M}$ sodium carbonate solution were added to $300 \mu \mathrm{L}$ of a suitably diluted aliquot of the extracts. The sample was maintained at $25^{\circ} \mathrm{C}$ in the dark for $10 \mathrm{~min}$, and then absorbance was measured at $655 \mathrm{~nm}$. The results were expressed as mmol Gallic acid equivalents/kg dry olive leaf (mmol GAE/kg).

The TFC of the olive leaves extracts was evaluated using the method described by Chang et al. [37] with modifications. In brief, $1 \mathrm{~mL}$ of extract was reconstituted to $1 \mathrm{~mL}$ with methanol-water $20 \%$ (v/v). After $5 \mathrm{~min}$ of incubation, $0.3 \mathrm{~mL}$ of $10 \%(\mathrm{w} / \mathrm{v}) \mathrm{AlCl}_{3}$ and $0.3 \mathrm{~mL} 5 \%(\mathrm{w} / \mathrm{v}) \mathrm{NaNO}_{2}$ solutions were added. The sample was allowed to stand for $6 \mathrm{~min}$. Then, $2 \mathrm{~mL}$ of a $1 \mathrm{M} \mathrm{NaOH}$ solution was added, and the final volume of the mixture was brought to $10 \mathrm{~mL}$ using double-distilled water. The sample was allowed to stand for $15 \mathrm{~min}$, and its absorbance was measured at $510 \mathrm{~nm}$. The TFC was evaluated from a calibration curve. The results were expressed as mmol Rutin equivalents $/ \mathrm{kg}$ dry olive leaf ( $\mathrm{mmol} \mathrm{RE} / \mathrm{kg}$ ).

\subsection{Antioxidant Capacities of Olive Leaves Extracts}

The DPPH free-radical scavenging method described by Vázquez-Roncero et al. [38] was used for determining the antioxidant capacity of the extracts. The inhibition percentage of the DPPH radicals was measured at $515 \mathrm{~nm}$ after $15 \mathrm{~min}$ in accordance with the following Equation (2):

$$
\% \text { DPPHinhibition }=\frac{[D P P H] \text { control }-[D P P H] \text { extract }}{[D P P H] \text { control }} 100
$$

The DPPH concentrations ([DPPH]) were calculated by interpolating the calibration curve of absorbance versus DPPH concentration. The inhibition percentage was transformed into antioxidant capacity using Trolox as external standard. For this purpose, another calibration curve graphing the 
percentage of inhibition against the Trolox concentration was used. The antioxidant capacity of the extracts was expressed as mmol Trolox equivalents/kg dry olive leaf (mmol TE/kg).

\subsection{Analysis of Phenolic Compounds by HPLC-DAD}

The determination of oleuropein and luteolin-7-glucoside in olive leaves extracts were carried out in accordance with the COI method (COI/T.20/Doc No 29/Rev.1, 2017) for the determination of biophenols with modifications. HPLC quantification was performed on a Shimadzu Prominence UFLC chromatograph system equipped with an autosampler, a column oven, a degasser, a diode array detector, and a quaternary pump. A C18 reverse-phase column ( $250 \mathrm{~mm} \times 4.6 \mathrm{~mm})$, type BDS HYPERSIL $5 \mu \mathrm{m}$ (Thermo Fisher Scientific Inc., USA) was used at $30^{\circ} \mathrm{C}$ to separate the biophenols. Oleuropein and luteolin-7-glucoside were identified by comparison with their commercial standards through retention times and the UV absorption spectra in the 190-350 nm range. Quantification was carried out by integration of the peaks at $280 \mathrm{~nm}$ using five-point regression curves of individual stock solutions of known concentration in triplicate of external standards. The phenolic compounds contents were expressed as $\mathrm{g}$ polyphenol/kg dry olive leaves ( $\mathrm{g}$ polyphenol/ $\mathrm{kg}$ ).

\section{Results and Discussion}

\subsection{Influence of UAE Factors on the Extraction of Bioactive Compounds from Olive Leaves}

Extraction procedure is a very important stage for the recovery and isolation of bioactive compounds from olive leaves. Although the effect of UAE operating conditions on the extraction yield, total phenolic and flavonoid contents, and antioxidant capacity has been studied, few works have reported the influence of extraction factors on the recovery of specific phenolic compounds from olive leaves $[23,25,33]$ using UAE and RSM, at least from our knowledge of literature, and only one of them tackled the task of how to simultaneously optimize the recovery of some of the main phenolic compounds found in olive leaves extracts (verbascoside and luteolin-4'-O-glucoside) besides oleuropein, but without taking into account amplitude as a factor to study [39]. In order to obtain the maximum extraction yield of oleuropein and luteolin-7-glucoside, two of the key factors involved in the UAE technique such as the increment of temperature, or maximum operating temperature, and amplitude were examined in this study. CCD and RSM were used to perform the tests and interpret the experimental results and optimize the operating conditions of this technique. The experimental design is presented in Table 1. The time duration of the ultrasonic processing needed to reach the maxima temperatures was measured and can be seen in Table 2.

Table 2. Responses for the central composite design obtained of the extractions by UAE ${ }^{1}$.

\begin{tabular}{|c|c|c|c|c|c|c|c|}
\hline Run & $\begin{array}{l}\text { Ultrasonic } \\
\text { Time (min) }\end{array}$ & $\begin{array}{c}\mathrm{R} \\
(\mathrm{g} / \mathrm{kg})\end{array}$ & $\begin{array}{c}\text { TPC (mmol } \\
\text { GAE/kg) }\end{array}$ & $\begin{array}{c}\text { TFC (mmol } \\
\text { RE/kg) }\end{array}$ & $\begin{array}{c}\text { DPPH (mmol } \\
\text { TE/kg) }\end{array}$ & $\begin{array}{l}\text { Oleuropein } \\
(\mathrm{g} / \mathrm{kg})\end{array}$ & $\begin{array}{l}\text { Luteolin-7- } \\
\text { Glucoside }(\mathrm{g} / \mathrm{kg})\end{array}$ \\
\hline 1 & 5.05 & $244.2^{1}$ & 178.1 & 211.0 & 182.9 & 58.2 & 1.46 \\
\hline 2 & 14.45 & 242.1 & 180.4 & 211.8 & 179.6 & 58.8 & 1.51 \\
\hline 3 & 2.00 & 190.7 & 146.1 & 170.2 & 146.2 & 47.3 & 1.15 \\
\hline 4 & 3.00 & 227.9 & 160.1 & 191.3 & 158.1 & 52.3 & 1.31 \\
\hline 5 & 17.93 & 292.9 & 212.8 & 261.0 & 213.8 & 71.5 & 1.87 \\
\hline 6 & 2.42 & 188.5 & 138.7 & 157.8 & 135.9 & 44.9 & 1.08 \\
\hline 7 & 5.13 & 256.0 & 186.3 & 223.4 & 183.2 & 62.2 & 1.56 \\
\hline 8 & 5.55 & 248.1 & 177.9 & 212.1 & 175.0 & 58.9 & 1.46 \\
\hline 9 & 5.82 & 209.5 & 156.3 & 185.6 & 156.8 & 51.0 & 1.26 \\
\hline 10 & 9.27 & 312.4 & 215.9 & 266.1 & 214.6 & 71.6 & 1.88 \\
\hline 11 & 4.98 & 283.5 & 197.2 & 239.4 & 198.8 & 65.3 & 1.67 \\
\hline 12 & 5.05 & 247.6 & 182.8 & 218.7 & 185.5 & 60.1 & 1.50 \\
\hline 13 & 5.07 & 221.5 & 164.3 & 195.9 & 166.2 & 52.3 & 1.33 \\
\hline
\end{tabular}


Table 2 also summarizes the influence of temperature and amplitude on R, TPC, TFC, DPPH, and oleuropein and luteolin-7-glucoside contents of olive leaves extracts by UAE and shows experimental values obtained all of the responses for each the design runs. The oleuropein content ranged from $44.9 \mathrm{~g} / \mathrm{kg}$ to $71.6 \mathrm{~g} / \mathrm{kg}$ dry leaf. As for luteolin-7-glucoside content, the quantity changed between $1.08 \mathrm{~g} / \mathrm{kg}$ and $1.88 \mathrm{~g} / \mathrm{kg}$ dry leaf. The highest values of oleuropein and luteolin-7-glucoside (runs 5 and 10) was in agreement with the best results of TPC, TFC, and DPPH, showing that these polyphenols can also be the main compounds responsible for the antioxidant capacity of the obtained extracts.

Increment of Temperature and Amplitude Influence

The mathematical models based on the experimental results for each of the responses are showed in the Table 3. Analysis of variance in accordance with the p-values stated in Section 2.3 showed that the models were reliable for prediction of responses.

Table 3. Mathematical models displayed in coded factors describing the behavior of the responses, including the main statistical parameters.

\begin{tabular}{cccc}
\hline Response & Models & $\mathbf{R}^{2}$ & CV (\%) \\
\hline Time $(\mathrm{min})$ & $5.17+3.64 \cdot \Delta \mathrm{T}-4.12 \cdot \mathrm{A}-2.28 \cdot \Delta \mathrm{TA}+1.01 \cdot \Delta \mathrm{T}^{2}+1.69 \mathrm{~A}^{2} \pm 0.33$ & $0.997^{1}$ & $5.13^{2}$ \\
$\mathrm{R}(\mathrm{g} / \mathrm{kg})$ & $249.86+44.00 \Delta \mathrm{T}-6.05 \cdot \mathrm{A}-6.87 \cdot \mathrm{A}^{2} \pm 3.82$ & 0.992 & 1.56 \\
TPC $(\mathrm{mmol} \mathrm{GAE} / \mathrm{kg})$ & $180.53+27.13 \cdot \Delta \mathrm{T}-6.83 \cdot \mathrm{A}-4.24 \cdot \mathrm{A}^{2} \pm 3.55$ & 0.984 & 2.00 \\
TFC (mmol RE/kg) & $216.01+37.27 \cdot \Delta \mathrm{T}-8.25 \cdot \mathrm{A}-5.47 \cdot \mathrm{A}^{2} \pm 5.43$ & 0.980 & 2.56 \\
DPPH (mmol TE/kg) & $180.48+27.66 \cdot \Delta \mathrm{T}-7.00 \cdot \mathrm{A}-4.41 \cdot \mathrm{A}^{2} \pm 4.75$ & 0.973 \\
Oleuropein $(\mathrm{g} / \mathrm{kg})$ & $59.60+9.55 \cdot \Delta \mathrm{T}-2.39 \cdot \mathrm{A}-1.63 \cdot \mathrm{A}^{2} \pm 1.53$ & 2.67 \\
Luteolin-7-glucoside $(\mathrm{g} / \mathrm{kg})$ & $1.50+0.28 \cdot \Delta \mathrm{T}-0.074 \cdot \mathrm{A}-0.033 \cdot \mathrm{A}^{2} \pm 0.040$ & 0.977 & 2.61 \\
\hline
\end{tabular}

${ }^{1}$ Coefficient of determination; ${ }^{2}$ Coefficient of variation.

When setting the amplitude and the temperature increase as operating factors, the operating time is variable and is interpreted as response, so that the model in Table 3 shows the existing interrelation between the three variables. As it is deduced from the model, the time increases with $\Delta \mathrm{T}$ and decreases with the amplitude and shows a small positive curvature with both factors. In addition, there is a negative interaction between the factors so that at the lower ends of one factor the action of the other increases. Thus, for $\mathrm{A}=-1$ the coefficient of $\Delta \mathrm{T}$ becomes +5.92 and for $\mathrm{A}=+1$ its value is +1.36 , which indicates that for low amplitudes the heating speed is low and for high amplitudes the speed is high (Figure 1). Likewise, for the amplitude, if $\Delta \mathrm{T}$ is set at its value -1 the coefficient of $\mathrm{A}$ is -1.84 , the time slowly decreases with the increase of $\mathrm{A}$, and for $\Delta \mathrm{T}=+1$ the coefficient is -6.40 , the decrease in time is considerably higher than in the previous case. The greater the amplitude, the higher the heating rate and the shorter the time needed to reach a certain temperature.

For the rest of the responses in the Table 3, both increment of temperature and amplitude were statistically significant factors. The effects of interaction between the factors did not show to be statistically significant. As it can be seen, only linear terms and quadratic effect of amplitude resulted as significant. These data are quite consistent with those reported in a previous work [23] in which the quadratic terms of the amplitude were significant for the responses TPC and TFC measured in olive mill leaves extracts. In all models, except for time, $\Delta \mathrm{T}$ is the most important factor by several orders of magnitude. This indicates that, for these responses, it is much more important to increase temperature than to decrease amplitude, although with low amplitudes the responses are improved.

Since time and temperature are dependant on each other in UAE, optimizing the increment of temperature and/or maximum temperature makes it possible to reduce extraction time. In the case of UAE, in general, it has been reported that an increase in temperature from 20 to $70{ }^{\circ} \mathrm{C}$ correlates with enhancements in extraction yields of the phenolic compounds from olive leaves as well as occurs in other biomass compared to non-ultrasonicated extractions [22]. This is owing to the induction of matrix bonds ruptures, the increase of the compound solubility, solvent diffusion rate, mass transfer, and reduction in viscosity of the solvent [31]. Effectively, in this work a positive correlation was observed between all responses and the increment of temperature, which was the most influential 
factor, indicated that the responses were directly proportional to the temperature reached. A similar positive effect of temperature on total polyphenols recovery and antioxidant capacity from olive leaves has been also described by other authors $[3,25]$.

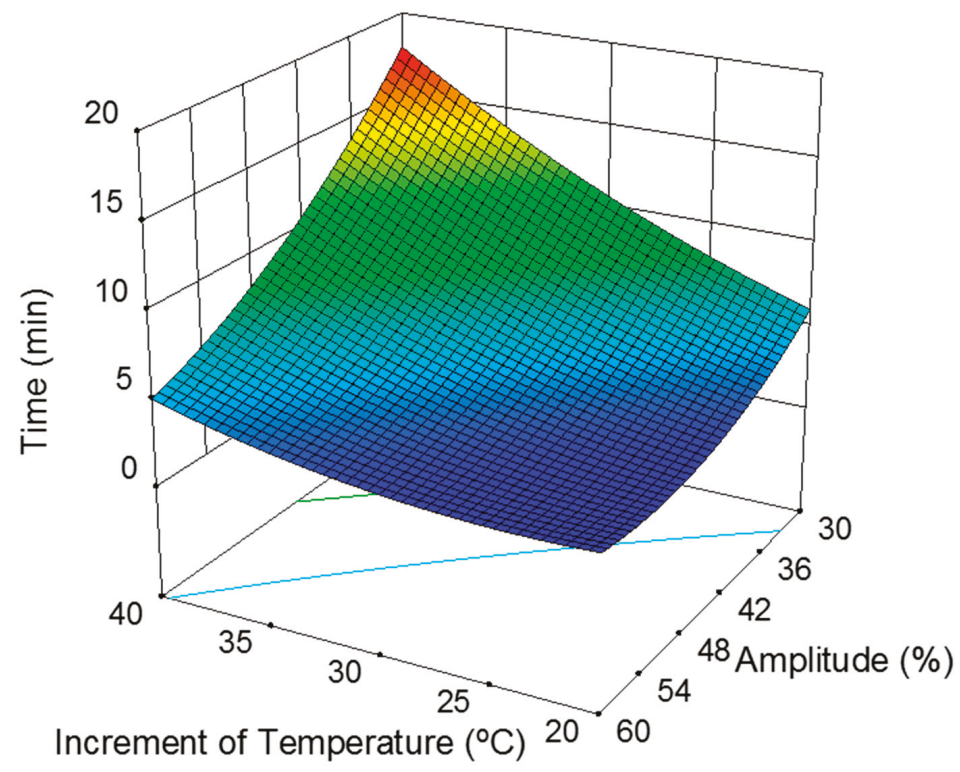

Figure 1. Variation of ultrasonic process duration time with increment of temperature and amplitude during processing of olive leaves by UAE.

In the ultrasonic system used in this work, the system with a coupled probe, amplitude plays an important role in the extraction since the number of compression cycles of ultrasonics depends on it. However, in this work its influence was less significant and showed a negative correlation with all responses slightly decreasing with increasing amplitude. It was observed that high amplitudes resulted in solvent agitation instead of cavitation, and as a consequence in low transmission of the ultrasounds through the liquid media.

The analysis of the simultaneous effects of the independent factors on the responses can also be seen on 3D response surface curves. Response surface plots were constructed in accordance with the equations of the predicted models (Table 3). Figure 2 presents the response surface graphs showing the combined influence of increment of temperature and amplitude on the oleuropein and luteolin-7-glucoside contents. Increment of temperature during extraction process was the most significant factor and the responses linearly increased with raising temperature, separately from amplitude.

\subsection{Optimization of the UAE Conditions}

The extraction process was optimized by using RSM. A simultaneous numerical optimization of the experimental conditions for all of responses was carried out using the optimization option in Design Expert software to maximize the responses what generated as optimal conditions of increment of temperature and amplitude of $40^{\circ} \mathrm{C}$ (corresponding to a maximum temperature of $60{ }^{\circ} \mathrm{C}$ ) and $30 \%$ (minimum amplitude), respectively (Table 4). 


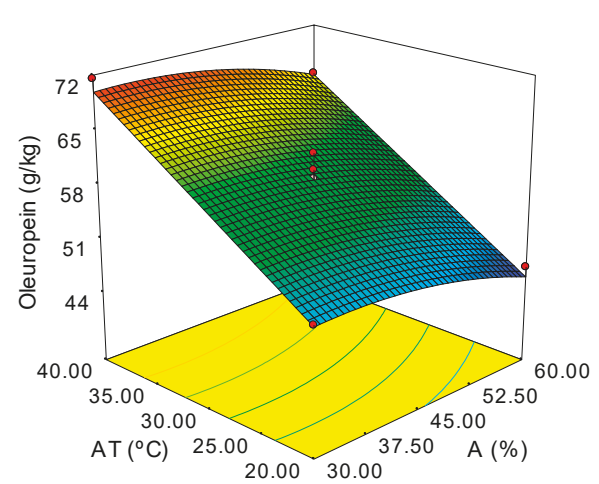

(a)

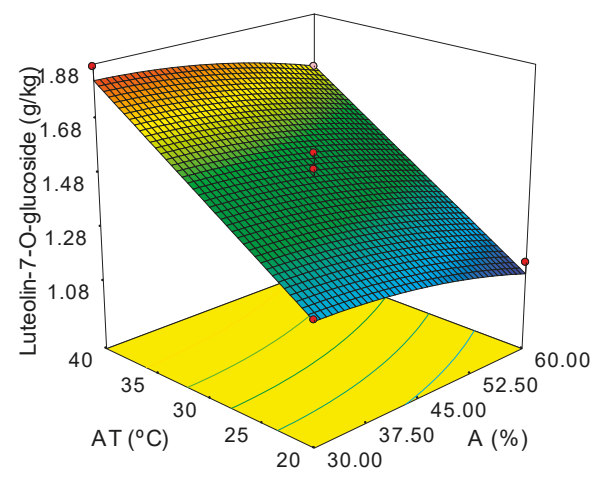

(b)

Figure 2. Response surface analysis for the oleuropein and luteolin-7-glucoside contents from olive leaves extracts with ultrasound-assisted extraction describing the influence of the factors increment of temperature and amplitude. The maxima of the responses are located at $60{ }^{\circ} \mathrm{C}$ and $30 \%$ amplitude. (a) Model graph for oleuropein content; (b) Model graph for luteolin-7-glucoside content.

Table 4. Comparison of both extraction methods applied in this work.

\begin{tabular}{ccc}
\hline Response & Soxhlet Extraction & Ultrasound-Assisted Extraction $^{\mathbf{1}}$ \\
\hline Time $(\mathrm{min})$ & 240 & $17.91 \pm 0.33$ \\
$\mathrm{R}(\mathrm{g} / \mathrm{kg})$ & $368.77 \pm 0.14^{2}$ & $293.06 \pm 3.82$ \\
$\mathrm{TPC}(\mathrm{mmol} \mathrm{GAE} / \mathrm{kg})$ & $251.91 \pm 2.13$ & $210.25 \pm 3.55$ \\
$\mathrm{TFC}(\mathrm{mmol} \mathrm{RE} / \mathrm{kg})$ & $274.97 \pm 4.39$ & $256.07 \pm 5.43$ \\
DPPH $(\mathrm{mmol} \mathrm{TE} / \mathrm{kg})$ & $291.42 \pm 18.80$ & $210.73 \pm 4.75$ \\
Oleuropein $(\mathrm{g} / \mathrm{kg})$ & $65.57 \pm 0.70$ & $69.91 \pm 1.53$ \\
Luteolin-7-glucoside $(\mathrm{g} / \mathrm{kg})$ & $1.32 \pm 0.03$ & $1.82 \pm 0.04$ \\
\hline
\end{tabular}

${ }^{1}$ Optimal values resulted from a simultaneous numerical optimization all of the responses, including time; ${ }^{2}$ Values reported are averages. The results were expressed as means \pm standard deviation of replicate extractions in triplicate.

Other researchers have also proven that the maximum oleuropein content is obtained at an extraction optimal temperature of $60{ }^{\circ} \mathrm{C}[33,39]$. Using optimal conditions, the maxima response values R, TPC, TFC, DPPH, oleuropein and luteolin-7-glucoside contents, and time were theoretically calculated (Table 4). The use of temperatures over the optimum should be avoided owing to the fact they lead to solvent loss by evaporation and may also promote degradation of the compounds of interest which has been reported at higher temperatures and therefore for longer periods of time using UAE [40]. As for the other factor considered for optimization, 30\% of amplitude was also selected as an optimal value by other authors [35] which indicated significant statistical differences when comparing phenolic extraction yields utilizing amplitudes in a range of $20 \%-50 \%$ [41]. Also, as mentioned before, it is important to indicate that high amplitudes can deteriorate the ultrasonic probe, resulting in liquid agitation instead of agitation.

\subsection{Comparison against Soxhlet Conventional Extraction Method}

Phenolic profiles from olive leaves extracts obtained by UAE and Soxhlet are shown in Figure 3. As can be seen, the extracts did not exhibit significant differences in composition, showing similar chromatograms as for the types of compounds observed in each one, although the amounts recovered were different. Oleuropein and luteolin-7-glucoside were the most abundant phenolic and flavonoid compounds in olive leaves extracts. 


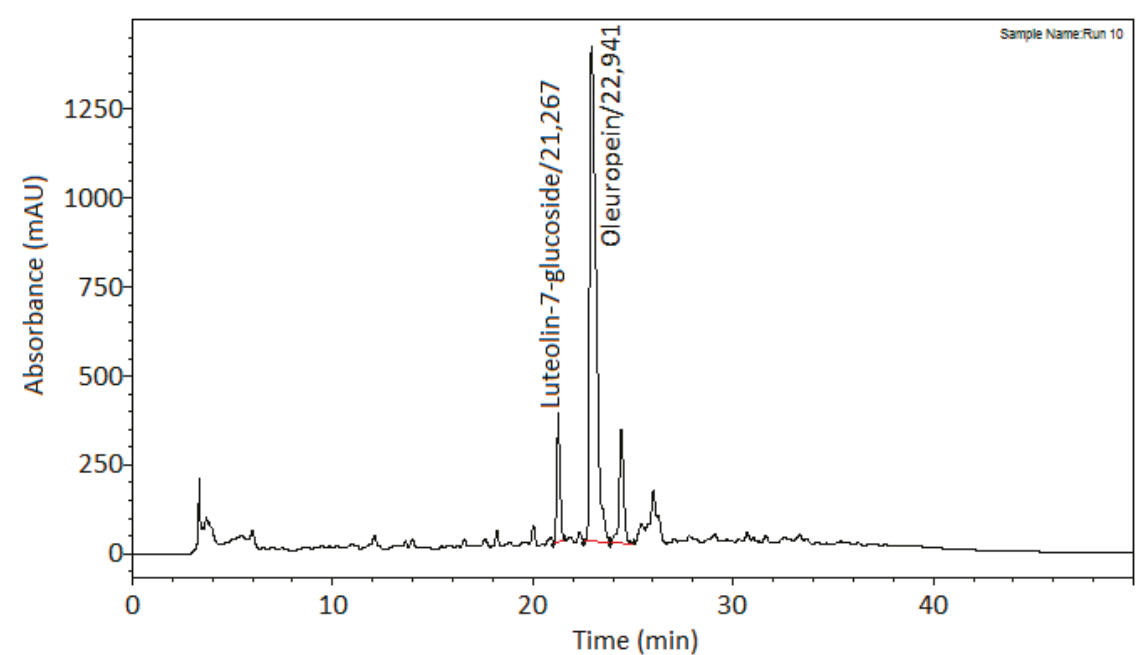

(a)

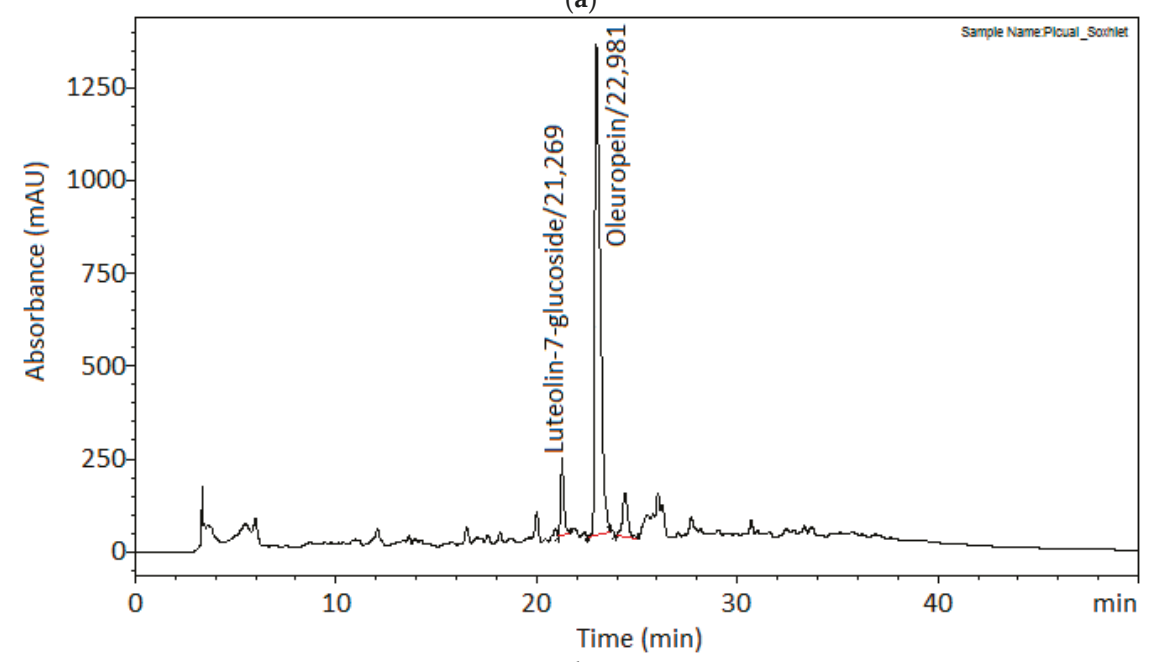

(b)

Figure 3. Representatives HPLC chromatograms recorded at $280 \mathrm{~nm}$ of olive leaves extracts recovered by UAE under the following conditions: $44.1^{\circ} \mathrm{C}$ of $\Delta \mathrm{T}$ and amplitude $45 \%$ (a) and Soxhlet extraction for $4 \mathrm{~h}$; (b) the peaks of oleuropein and luteolin-7-glucoside appear marked in the chromatograms.

Table 4 shows a comparison of the responses obtained by conventional Soxhlet extraction under the conditions described in the Section 2.2 and UAE from a simultaneous numerical optimization all of the responses obtained with an operating conditions of $44.1^{\circ} \mathrm{C}$ of $\Delta \mathrm{T}$ and $30 \%$ of amplitude from CCD stated in Table 1 . The best results regarding R, TPC, TFC, and DPPH were reached by Soxhlet method. Based on the differences observed between UAE and Soxhlet extracts in R, TPC, and TFC, it is reasonable to think that Soxhlet extracts may contain other phenolics in higher concentrations than oleuropein and luteolin-7-glucoside but also having solubilized more impurities during the extraction. On the other hand, ultrasounds can ease swelling and hydration, resulting in an expansion in the pores of the cell wall, which enhances the diffusion action, leading to improve mass transfer. However, the second stage which is the limiting step requires additional energy, for example, stirring to quicken 
the extraction procedure. The ultrasonic breakdown of olive leaf matrix by ultrasonics might not be enough. In addition, in the Soxhlet method each cycle of extraction was done with pure solvent which can be another explanation of the better yield of Soxhlet over UAE [42] in these responses.

The extraction method had also a great effect on the amount of the bioactives quantified in this work. In this case, however, if the influence of extraction method on oleuropein and luteolin-7-glucoside contents in the extracts is analyzed, the data indicate that the same trend does not repeat because UAE provides significantly higher contents of both compounds compared to Soxhlet extraction. In the case of oleuropein, UAE extracts $(69.91 \mathrm{~g} / \mathrm{kg})$ showed a $6.6 \%$ significantly higher content than Soxhlet extracts ones $(65.57 \mathrm{~g} / \mathrm{kg})$. With regards to the luteolin-7-glucoside content, significant differences were also found between Soxhlet $(1.32 \mathrm{~g} / \mathrm{kg})$ and UAE extracts $(1.82 \mathrm{~g} / \mathrm{kg})$. UAE extracts exhibited a $37.9 \%$ higher content than Soxhlet ones. Long exposure to temperature for several hours can more likely result in the degradation of these compounds by Soxhlet method. These differences were observed by other researchers; for instance, Cifá et al. [23] optimized the extraction of oleuropein and reported significant increases in extraction yield of oleuropein from $6 \%$ to $84 \%$ compared to traditional maceration and therefore within the range obtained in this work. Several works show the efficiency of the UAE technique compared to conventional methods. In any case, with regards to the content of the quantified bioactive compounds and from a point of view of the purity of the extracts, this should be seen as an advantage in favor of ultrasound-assisted extraction, since in the end purer extracts in oleuropein and luteolin-7-glucoside were obtained.

Although there are many aspects that can influence the extracts composition, like the cultivar and sampling date, the oleuropein content reached in this work was similar than that reported by other authors utilizing the same extraction technique, i.e., ultrasound application. The maximum oleuropein content was $71.65 \mathrm{~g} / \mathrm{kg}$ dry olive leaf versus $65 \mathrm{~g} / \mathrm{kg}$ dry olive leaf determined by Ahmad-Qasem et al. [43].

\section{Conclusions}

During the last decade, the interest in the recovery of bioactive polyphenols from olive leaves has increased in the industry and the scientific community. Oleuropein and luteolin-7-glucoside extraction from olive leaves could add value to this by-product of the olive oil industry if they are recovered by applying efficient extraction technologies. The present work investigated the influence of UAE experimental conditions (increment of temperature and amplitude) on the extraction yield of the two main olive leaf polyphenols, showing a significant increase of oleuropein and luteolin-7-glucoside contents in the extracts $(6.6 \%$ and $37.9 \%$, respectively) in comparison to Soxhlet conventional extraction. The extraction samples are heated using ultrasound, so that the greater the amplitude, the higher the heating rate. The optimal results were obtained at low amplitudes and high temperatures. The highest extraction efficiency and antioxidant capacity were obtained under optimal increment of temperature and amplitude conditions ( $40{ }^{\circ} \mathrm{C}$ and $30 \%$ amplitude). Under these conditions, the simultaneous optimization of oleuropein and luteolin-7-glucoside provided a concentration of oleuropein of $69.91 \mathrm{~g} / \mathrm{kg}$ whilst the concentration of luteolin-7-glucoside was $1.82 \mathrm{~g} / \mathrm{kg}$. The results demonstrated that the UAE is quicker and more efficient than conventional Soxhlet method, providing olive leaves extracts with higher amounts of oleuropein and luteolin-7-glucoside, but significantly reducing the extraction time (around only $15 \mathrm{~min}$ are required versus $4 \mathrm{~h}$ of the traditional extraction) and energy costs. This work can help to approach new studies on the UAE process on a larger scale for later industrial valorization of olive leaves. This process could provide large enough amounts of the bioactive compounds studied which will allow more analyses of their biological activities and improvement in the understanding of their properties.

Author Contributions: Conceptualization, A.L.M., F.E. and M.M.; methodology, A.L.M. and M.d.M.C.; software, M.M.; writing - original draft preparation, A.L.M and M.d.M.C.; writing—review and editing, A.L.M., F.E. and M.M.; supervision, E.C.; project administration, F.E.; funding acquisition, A.L.M., F.E. and I.R. 
Funding: This research was funded by University of Jaén (Acción 6) and Ministerio de Ciencia, Innovación y Universidades of Spanish Government (Project code: ENE2017-85819-C2-1-R).

Acknowledgments: The authors want to thank the technical and human support provided by CICT of the University of Jaén (UJA, MINECO, Junta de Andalucía, FEDER).

Conflicts of Interest: The authors declare no conflict of interest.

\section{References}

1. Rahmanian, N.; Jafaric, S.M.; Wani, T.A. Bioactive profile, dehydration, extraction and application of the bioactive components of olive leaves. Trends Food Sci. Technol. 2015, 42, 150-172. [CrossRef]

2. Cara, C.; Romero, I.; Oliva, J.M.; Sáez, F.; Castro, E. Liquid hot water pretreatment of olive tree pruning residues. Appl. Biochem. Biotechnol. 2007, 136-140, 379-394.

3. Xie, P.; Huang, L.; Zhang, C.; Zhang, Y. Phenolic compositions, and antioxidant performance of olive leaf and fruit (Olea europaea L.) extracts and their structure-activity relationships. J. Funct. Foods 2015, 16, 460-471. [CrossRef]

4. Luque de Castro, M.D.; Priego Capote, F. Extraction of oleuropein and related phenols from olive leaves and branches. In Olives and Olive Oil in Health and Disease Prevention, 1st ed.; Preedy, V., Watson, R., Eds.; Elsevier: Amsterdam, The Netherlands, 2010; Volume 1, pp. 259-273.

5. Rodrigues, F.; Pimentel, F.B.; Oliveira, M.B.P.P. Olive by-products: Challenge application in cosmetic industry. Ind. Crop. Prod. 2015, 70, 116-124. [CrossRef]

6. Nunes, M.A.; Pimentel, F.B.; Costa, A.S.G.; Alves, R.C.; Oliveira, M.B.P.P. Olive by-products for functional and food applications: Challenging opportunities to face environmental constraints. Innov. Food Sci. Emerg. 2016, 35, 139-148. [CrossRef]

7. Žugčić, T.; Abdelkebir, R.; Alcantara, C.; Collado, M.C.; García-Pérez, J.V.; Meléndez-Martínez, A.J.; Jambrak, A.R.; Lorenzo, J.M.; Barba, F.J. From extraction of valuable compounds to health promoting benefits of olive leaves through bioaccessibility, bioavailability and impact on gut microbiota. Trends Food Sci. Technol. 2019, 83, 63-77. [CrossRef]

8. Özcan, M.M.; Matthäus, B. A review: Benefit and bioactive properties of olive (Olea europaea L.) leaves. Eur. Food Res. Technol. 2017, 243, 89-99. [CrossRef]

9. Vogel, P.; Machado, I.K.; Garavaglia, J.; Zani, V.T.; de Souza, D.; Dal Bosco, S.M. Polyphenols benefits of olive leaf (Olea europaea L.) to human health. Nutr. Hosp. 2015, 31, 1427-1433. [CrossRef]

10. Şahin, S.; Elhussein, E.; Bilgin, M.; Lorenzo, J.M.; Barba, F.J.; Roohinejad, S. Effect of drying method on oleuropein, total phenolic content, flavonoid content, and antioxidant activity of olive (Olea europaea) leaf. J. Food Process. Pres. 2018, 1-10. [CrossRef]

11. Talhaoui, N.; Taamalli, A.; Gómez-Caravaca, A.M.; Fernández-Gutiérrez, A.; Segura-Carretero, A. Phenolic compounds in olive leaves: Analytical determination, biotic and abiotic influence, and health benefits. Food Res. Int. 2015, 70, 92-108. [CrossRef]

12. Salem, M.B.; Affes, H.; Ksouda, K.; Sahnoun, Z.; Zeghal, K.M.; Hammami, S. Pharmacological activities of Olea europaea leaves. J. Food Process. Pres. 2015, 39, 3128-3136. [CrossRef]

13. Hassen, I.; Casabianca, H.; Hosni, K. Biological activities of the natural antioxidant oleuropein: Exceeding the expectation-A mini-review. J. Funct. Foods 2015, 18, 926-940. [CrossRef]

14. Samet, I.; Villareal, M.O.; Motojima, H.; Han, J.; Sayadi, S.; Isoda, H. Olive leaf components apigenin 7-glucoside and luteolin 7-glucoside direct human hematopoietic stem cell differentiation towards erythroid lineage. Differentiation 2015, 89, 146-155. [CrossRef]

15. Ahmad-Qasem, M.H.; Cánovas, J.; Barrajón-Catalán, E.; Carreres, J.E.; Micol, V.; García-Pérez, J.V. Influence of olive leaf processing on the bioaccessibility of bioactive polyphenols. J. Agric. Food Chem. 2014, 62, 6190-6198. [CrossRef]

16. Cruz, R.M.S.; Brito, R.; Smirniotis, P.; Nikolaidou, Z.; Vieira, M.C. Extraction of bioactive compounds from olive leaves using emerging technologies. In Ingredients Extraction by Physicochemical Methods in Food, 1st ed.; Grumezescu, A., Holban, A.-M., Eds.; Elsevier Inc.: Amsterdam, The Netherlands, 2017; Volume 4, pp. 441-461.

17. Daso, A.P.; Okonkwo, O.J. Conventional extraction techniques: Soxhlet and liquid-liquid extractions and evaporation. Anal. Sep. Sci. 2015, 5, 1437-1468. [CrossRef] 
18. Lama-Muñoz, A.; Contreras, M.M.; Espínola, F.; Moya, M.; de Torres, A.; Romero, I.; Castro, E. Extraction of oleuropein and luteolin-7-O-glucoside from olive leaves: Optimization of technique and operating conditions. Food Chem. 2019, 293, 161-168. [CrossRef]

19. Šoštarič, M.; Klinar, D.; Bricelj, M.; Golob, J.; Berovič, M.; Likozar, B. Growth, lipid extraction and thermal degradation of the microalga Chlorella vulgaris. New Biotechnol. 2012, 29, 325-331. [CrossRef]

20. Borić, M.; Puliyalil, H.; Novak, U.; Likozar, B. An intensified atmospheric plasma-based process for the isolation of the chitin biopolymer from waste crustacean biomass. Green Chem. 2018, 20, 1199-1204. [CrossRef]

21. Bajić, M.; Jalšovec, H.; Travan, A.; Novak, U.; Likozar, B. Chitosan-based films with incorporated supercritical $\mathrm{CO}_{2}$ hop extract: Structural, physicochemical, and antibacterial properties. Carbohydr. Polym. 2019, 219, 261-268. [CrossRef]

22. Chemat, F.; Rombaut, N.; Sicaire, A.-G.; Meullemiestre, A.; Fabiano-Tixier, A.-S.; Abert-Vian, M. Ultrasound assisted extraction of food and natural products. Mechanisms, techniques, combinations, protocols and applications. A review. Ultrason. Sonochem. 2017, 34, 540-560. [CrossRef]

23. Cifá, D.; Skrt, M.; Pittia, P.; Di Mattia, C.; Ulrih, N.P. Enhanced yield of oleuropein from olive leaves using ultrasound-assisted extraction. Food Sci. Nutr. 2018, 6, 1128-1137. [CrossRef]

24. Shirzad, H.; Niknam, V.; Taheri, M.; Ebrahimzadeh, H. Ultrasound-assisted extraction process of phenolic antioxidants from Olive leaves: A nutraceutical study using RSM and LC-ESI-DAD-MS. J. Food Sci. Technol. 2017, 54, 2361-2371. [CrossRef]

25. Irakli, M.; Chatzopoulou, P.; Ekateriniadou, L. Optimization of ultrasound-assisted extraction of phenolic compounds: Oleuropein, phenolic acids, phenolic alcohols and flavonoids from olive leaves and evaluation of its antioxidant activities. Ind. Crop. Prod. 2018, 124, 382-388. [CrossRef]

26. Martínez-Patiño, J.C.; Gullón, B.; Romero, I.; Ruiz, E.; Brnčić, M.; Žlabur, J.Š.; Castro, E. Optimization of ultrasound-assisted extraction of biomass from olive trees using response surface methodology. Ultrason. Sonochem. 2019, 51, 487-495. [CrossRef]

27. Goldsmith, C.D.; Vuong, Q.V.; Stathopoulos, C.E.; Roach, P.D.; Scarlett, C.J. Ultrasound increases the aqueous extraction of phenolic compounds with high antioxidant activity from olive pomace. LWT-Food Sci. Technol. 2018, 89, 284-290. [CrossRef]

28. Roselló-Soto, E.; Koubaa, M.; Moubarik, A.; Lopes, R.P.; Saraiva, J.A.; Boussetta, N.; Grimi, N.; Barba, F.J. Emerging opportunities for the effective valorization of wastes and by-products generated during olive oil production process: Non-conventional methods for the recovery of high-added value compounds. Trends Food Sci. Technol. 2015, 45, 296-310. [CrossRef]

29. Di Khanh, N. Advances in the extraction of anthocyanin from vegetables. J. Food Nutr. Sci. 2015, 3, $126-134$. [CrossRef]

30. Arabi, M.; Ghaedi, M.; Ostovan, A. Development of dummy molecularly imprinted based on functionalized silica nanoparticles for determination of acrylamide in processed food by matrix solid phase dispersion. Food Chem. 2016, 210, 78-84. [CrossRef]

31. Medina-Torres, N.; Ayora-Talavera, T.; Espinosa-Andrews, H.; Sánchez-Contreras, A.; Pacheco, N. Ultrasound assisted extraction for the recovery of phenolic compounds from vegetable sources. Agronomy 2017, 7, 47. [CrossRef]

32. Şahin, S.; Şamlı, R. Optimization of olive leaf extract obtained by ultrasound-assisted extraction withresponse surface methodology. Ultrason. Sonochem. 2013, 20, 595-602. [CrossRef]

33. İlbay, Z.; Şahin, S.; Büyükkabasakal, K. A novel approach for olive leaf extraction through ultrasound technology: Response surface methodology versus artificial neural networks. Korean J. Chem. Eng. 2014, 31, 1661-1667. [CrossRef]

34. Wang, B.; Qu, J.; Luo, S.; Feng, S.; Li, T.; Yuan, M.; Huang, Y.; Liao, J.; Yang, R.; Ding, C. Optimization of ultrasound-assisted extraction of flavonoids from olive (Olea europaea) leaves, and evaluation of their antioxidant and anticancer activities. Molecules 2018, 23, 2513. [CrossRef]

35. Japón-Luján, R.; Luque-Rodríguez, J.M.; Luque de Castro, M.D. Dynamic ultrasound-assisted extraction of oleuropein and related biophenols from olive leaves. J. Chromatogr. A 2006, 1108, 76-82. [CrossRef]

36. Vidal, A.M.; Alcalá, S.; Ocaña, M.T.; De Torres, A.; Espínola, F.; Moya, M. Modeling of volatile and phenolic compounds and optimization of the process conditions for obtaining balanced extra virgin olive oils. Grasas Aceites 2018, 69, e250. [CrossRef] 
37. Chang, C.C.; Yang, M.H.; Wen, H.M.; Chern, J.C. Estimation of total flavonoid content in propolis by two complementary colorimetric methods. J. Food Drug Anal. 2002, 10, 178-182.

38. Vázquez-Roncero, A.; Janer del Valle, C.; Janer del Valle, M.L. Determinación de los polifenoles totales del aceite de oliva. Grasas Aceites 1973, 24, 350-357.

39. Giacometti, J.; Žauhar, G.; Žuvić, M. Optimization of ultrasonic-assisted extraction of major phenolic compounds from olive leaves (Olea europaea L.) using response surface methodology. Foods 2018, 7, 149. [CrossRef]

40. Ameer, K.; Shahbaz, H.M.; Kwon, J.-H. Green extraction methods for polyphenols from plant matrices and their byproducts: A review. Compr. Rev. Food Sci. Food Saf. 2017, 16, 295-315. [CrossRef]

41. Carrera, C.; Ruiz-Rodríguez, A.; Palma, M.; Barroso, C.G. Ultrasound assisted extraction of phenolic compounds from grapes. Anal. Chim. Acta 2012, 732, 100-104. [CrossRef]

42. Bilgin, M.; Şahin, S. Effects of geographical origin and extraction methods on total phenolic yield of olive tree (Olea europaea) leaves. J. Taiwan Inst. Chem. E. 2013, 44, 8-12. [CrossRef]

43. Ahmad-Qasem, M.H.; Cánovas, J.; Barrajón-Catalán, E.; Micol, V.; Cárcel, J.A.; García-Pérez, J.V. Kinetic and compositional study of phenolic extraction from olive leaves (var. Serrana) by using power ultrasound. Innov. Food Sci. Emerg. Technol. 2013, 17, 120-129. [CrossRef]

(C) 2019 by the authors. Licensee MDPI, Basel, Switzerland. This article is an open access article distributed under the terms and conditions of the Creative Commons Attribution (CC BY) license (http://creativecommons.org/licenses/by/4.0/). 
Article

\title{
Effect of Salt Addition upon the Production of Metabolic Compounds by Yarrowia lipolytica Cultivated on Biodiesel-Derived Glycerol Diluted with Olive-Mill Wastewaters
}

\author{
Markella Tzirita ${ }^{1}$, Maria Kremmyda ${ }^{1}$, Dimitris Sarris ${ }^{1,2}$, Apostolis A. Koutinas ${ }^{1}$ \\ and Seraphim Papanikolaou ${ }^{1, *}$ \\ 1 Department of Food Science \& Human Nutrition, Agricultural University of Athens, 75 Iera Odos, \\ 11855 Athens, Greece; markella.tz@gmail.com (M.T.); mariakremm1995@hotmail.com (M.K.); \\ akoutinas@aua.gr (A.K.) \\ 2 Department of Food Science \& Nutrition, School of the Environment, University of the Aegean, \\ 81400 Myrina, Lemnos, Greece; dsarris@aegean.gr \\ * Correspondence: spapanik@aua.gr; Tel.: +30-210-5294700
}

Received: 21 August 2019; Accepted: 16 September 2019; Published: 24 September 2019

\begin{abstract}
One of the major environmental problems is the highly toxic agro-industrial waste called olive mill wastewater (OMW), deriving from olive oil production. On the other hand, the continuous development of the biological liquid fuel industry (biodiesel and bioethanol) makes it mandatory the process and exploitation of their main by-products, crude glycerol. This study dealt with the biotechnological conversions of biodiesel-derived crude glycerol with the use of the non-conventional yeast Yarrowia lipolytica in media that had been diluted with OMWs. OMWs, employed as simultaneous liquid medium and substrate, is a new trend recently appearing in Industrial Biotechnology, where value-added metabolites could be produced with simultaneous partial detoxification (i.e. decolorization and phenol removal) of the used residue. In the present study, diluted OMWs (containing $2.0 \mathrm{~g} / \mathrm{L}$ of total phenolic compounds) blended with $70.0 \mathrm{~g} / \mathrm{L}$ crude glycerol were employed as substrates. Production of value-added compounds by Y. lipolytica strain ACA-YC 5031 was studied in nitrogen-limited media favoring the production of secondary metabolites (i.e. citric acid, polyols, microbial lipids, polysaccharides). Batch-flask cultures were carried out and the impact of the addition of different $\mathrm{NaCl}$ concentrations $(1.0 \%, 3.0 \%, 5.0 \% w / w)$ added upon the biochemical behavior of the strain was studied. Remarkable biomass production was observed in all trials, while in the "blank" experiment (no OMWs and no salt added), the metabolism was shifted toward the synthesis of polyols ( $\Sigma$ polyols $=$ mannitol + arabitol + erythritol $>20 \mathrm{~g} / \mathrm{L}$ and maximum total citric acid-Cit (sum of citric and isocitric acid) $=10.5 \mathrm{~g} / \mathrm{L}$ ). Addition of OMWs resulted in $\mathrm{Cit}_{\max }$ $=32.7 \mathrm{~g} / \mathrm{L}$, while $\Sigma$ polyols concentration dropped to $<15 \mathrm{~g} / \mathrm{L}$. Addition of salt in the OMW-based media slightly reduced the produced biomass, while Cit production drastically increased, reaching a final value of $54.0 \mathrm{~g} / \mathrm{L}$ (conversion yield of Cit produced per unit of glycerol consumed $=0.82 \mathrm{~g} / \mathrm{g}$ ) in the trial with addition of $5.0 \% \mathrm{NaCl}$. Finally, significant color and phenols removal were observed, evaluating the yeast as a decontamination medium for the OMW and a great candidate for the production of value-added compounds.
\end{abstract}

Keywords: Biodiesel-derived glycerol; citric acid; microbial lipid; olive-mill-wastewater; polyols; polysaccharides; Yarrowia lipolytica; value-added compounds 


\section{Introduction}

Olive-mill wastewaters (OMWs), the major effluent deriving from the olive oil production process, are considered as one of the most challenging agro-industrial wastes to treat, since they are produced in very high quantities. Moreover, their strong odor and dark color and their relatively high organic load have a direct negative impact on the environment if they are released untreated. This important residue of the olive oil industry is one of the most difficult to treat wastes because of its high content in phenolic compounds [1,2]. The increased concentration of OMWs (without prior treatment) in organic matter and phenolic components results in the reduction of the available concentration of oxygen to the organisms. Lack of oxygen to the organisms upsets the balance of ecosystems and the soil porosity, resulting in contaminated aquifers and polluted environments [1,3,4]. Consequent problems are the production of odors, the excessive growth of algae and bacteria, and the appearance of the eutrophication phenomena in aquatic environments in which OMWs are released untreated. The organic matter in OMW consists of sugars, phenols, tannins, polyphenols, aromatic molecules, ash, and, in some cases, lipids and nitrogen content [2]. The phenolic compounds are mainly considered responsible for the toxicity of the OMWs constituting, in several cases, the limiting step of their large-scale management [5-8]. It should be noted that, in several cases (i.e. in the case where traditional press extraction process is applied in order to liberate olive oil), OMWs containing very high concentrations of sugars (mostly glucose, in concentrations $\geq 65 \mathrm{~g} / \mathrm{L}$ [2]) are simultaneously liberated. However, a new trend that has appeared in relation to the valorization of these wastewaters refers to their simultaneous utilization as a substrate and water treatment in various fermentation processes. Value-added metabolites could be produced during these fermentation processes with simultaneous partial detoxification (i.e. decolorization and phenol removal) of the residue [2-4,9-11].

The continuous development of biological liquid fuel industry (principally that of biodiesel and bioethanol production) makes mandatory the process and exploitation of the increased production of its main by-product and principal liquid residue, which is the concentrated glycerol-containing water called crude glycerol (crude glycerin) [12]. Specifically concerning biodiesel production facilities, the synthesis of $10.0 \mathrm{~kg}$ of biodiesel deriving from trans-esterification of various oils, generates c. $1.0 \mathrm{~kg}$ of glycerol (purity $\approx 90.0 \% w / w$ ) $[13,14]$. Global biodiesel production has been growing in recent years. In 2016 more than $30.8 \times 10^{6} \mathrm{t}$. were produced ( $7.5 \%$ more than in 2015), and it is estimated that more than $3.0 \times 10^{6} \mathrm{t}$. of glycerol as an important industrial byproduct were produced only from biodiesel manufacture [15]. Biodiesel-derived glycerol is a great substrate for microbial growth due to its high concentration in carbon and inorganic constituents. Many studies have reported the ability of eukaryotic microorganisms to convert glycerol into a plethora of value-added compounds, such us microbial lipids (single cell oils; SCOs) [16-25], citric acid [11,26-32] polyols [20,33-35], enzymes, and microbial mass $[13,14,20,28,29,32,34,36-44]$. Biodiesel-derived glycerol as a carbon source employed in the Industrial Microbiology has many advantages over other conventional substrates frequently used as microbial substrates (i.e. commercial sugars). One such advantage is the significantly low (or even negative) acquisition cost that this renewable material presents. In spite of the fact that the industrial production of biodiesel is currently being accompanied by the side production of relatively highly purified industrial glycerol feedstock (e.g. purity of $\approx 85-90 \% w / w$, sometimes $>90 \% w / w$ ), which can be used directly as the starting material for the chemical synthesis of epichlorohydrin, or it can be used in various pharmaceutical applications after further purification processes. The acquisition cost of this relatively highly purified feedstock (i.e. purity $\approx 85-90 \% w / w$ ) is significantly low, and at the beginning of 2016, in the Asiatic markets, it was at c. $0.16 \mathrm{US} \$ / \mathrm{kg}$, with a systematic decreasing trend. Moreover, in several cases, a large fraction of low-quality glycerol deriving from biodiesel production process, the so-called "pitch" glycerol, is a typical waste-stream of the process amenable only for incineration, adding to climate relevant emissions $\left(\mathrm{CO}_{2}\right.$ as well as $\left.\mathrm{N}_{2} \mathrm{O}\right)$ and to the cost of the whole biodiesel production chain. Moreover, utilization of glycerol as microbial substrate is not competitive with substrates that can be used as edible products. It also has inorganic components such as potassium, calcium, sulfur and magnesium that can work favorably for microbial growth [45]. 
Y. lipolytica yeasts are ideal candidates for the remediation and valorization of OMWs as well as of other recalcitrant wastes and residues due to their ability to vigorously grow on a variety of substrates and to tolerate usually hostile growth media [46]. Moreover, Y. lipolytica yeasts have been reported to be capable of producing SCO having equivalent synthesis and value to that of cocoa-butter [47-50] and a plethora of other value-added compounds during growth on several types of agro-industrial co-products and residues of either fatty or hydrophilic chemical structures $[4,36,44,51,52]$. The present study investigated the ability of Y. lipolytica strain ACA-YC 5031, a strain that has been reported capable of producing SCO and citric acid during growth on glucose-based media under nitrogen-limited conditions [53], to grow and produce secondary metabolites on mixed nitrogen-limited media consisting of OMWs and biodiesel-derived glycerol. In fact, OMWs were used in order to partially replace tap water from the fermentations performed, whereas the effect of the addition of different sodium chloride $(\mathrm{NaCl})$ concentrations upon the physiological behavior of the strain on osmotic stress over the production of secondary metabolites was assessed. The number of investigations dealing with the cultivation of microorganisms on media that are composed of mixtures of OMWs with other low-cost substrates (i.e. commercial-type glucose, low-cost oils, etc.), in the approach in which OMWs are considered as simultaneous substrate and process water, are relatively restricted in the literature [3,8-11]. Moreover, studies in which blends of glycerol and OMWs as substrates were used are indeed very scarce [4]. On the other hand, the simultaneous effect of the addition of OMWs and $\mathrm{NaCl}$ upon the fermentation of glycerol by $\mathrm{Y}$. lipolytica strain, to the best of our knowledge, is studied for the first time in the literature. Therefore, the potential of a new natural Y. lipolytica strain (ACA-YC 5031) to reduce the color and the phenol content and simultaneously to convert the industrial wastes (OMWs blended with glycerol and $\mathrm{NaCl}$ ) into value-added compounds, useful in Industrial and Food Biotechnology, was investigated, and technological approaches of the bioprocess were critically addressed and discussed.

\section{Results}

\subsection{Batch Fermentations and Production of Value-Added Compounds by Yarrowia lipolytica Growing on $\mathrm{OMW} /$ Glycerol Blends Supplemented with $\mathrm{NaCl}$}

Experiments were carried out investigating the assimilation of glycerol and its conversion by the yeast $Y$. lipotytica ACA-YC 5031 to secondary metabolites with and without the addition of OMWs (phenolic compounds into the media $=2.0 \pm 0.20 \mathrm{~g} / \mathrm{L}$ ) into the medium. The effect on the microbial reactions and physiology of different concentrations of $\mathrm{NaCl}(1.0 \%, 3.0 \%$, and $5.0 \% w / v)$ added into the culture medium was also examined. Under all circumstances, there was production of secondary metabolites, such as SCO, citric acid, intracellular polysaccharides (IPS), and polyols (mainly mannitol and erythritol, while small concentrations of arabitol were also detected) during growth on OMW/glycerol blends, where variable quantities of $\mathrm{NaCl}$ were added. The secretion of extra-cellular metabolites was enhanced after virtual nitrogen depletion from the growth medium occurring c. 24-30 h after inoculation. Specifically, the initial nitrogen concentration of nitrogen as indicated by the concentration of extra-cellular free amino nitrogen (FAN) was $=85 \pm 10 \mathrm{mg} / \mathrm{L}$, whereas the final nitrogen concentration when this compound became the limiting substrate was $25 \pm 5 \mathrm{mg} / \mathrm{L}$ of FAN into the medium (data not presented). The results of the maximum values of the components obtained during the fermentations carried out are presented in Table 1. 
Table 1. values of glycerol consumption, production of biomass, cellular lipids, citric acid, mannitol, erythritol, intra-cellular polysaccharides (IPS) and yield coefficients in culture medium with just glycerol (blank) and glycerol blended with OMWs yielding at initial concentration of $2.0 \mathrm{~g} / \mathrm{L}$ of phenols using different concentrations of $\mathrm{NaCl} w / v(0.0 \%, 1.0 \%, 3.0 \%, 5.0 \%)$.

\begin{tabular}{|c|c|c|c|c|c|c|c|c|c|c|c|c|c|c|}
\hline \multicolumn{2}{|c|}{$\begin{array}{l}\text { Culture } \\
\text { medium }\end{array}$} & $\begin{array}{c}\text { Time } \\
\text { (h) }\end{array}$ & $\begin{array}{c}\mathrm{X} \\
(\mathrm{g} / \mathrm{L})\end{array}$ & $\begin{array}{c}\mathrm{L} \\
(\mathrm{g} / \mathrm{L})\end{array}$ & $\begin{array}{c}\text { Glol }_{\text {cons }} \\
(\mathrm{g} / \mathrm{L})\end{array}$ & $\begin{array}{c}\text { Cit } \\
(\mathrm{g} / \mathrm{L})\end{array}$ & $\begin{array}{l}\text { Man } \\
(\mathrm{g} / \mathrm{L})\end{array}$ & $\begin{array}{c}\text { Ery } \\
(\mathrm{g} / \mathrm{L})\end{array}$ & $\begin{array}{c}Y_{X / \text { Glol }} \\
(\mathrm{g} / \mathrm{g})\end{array}$ & $\begin{array}{l}Y_{L / X} \% \\
(w / w)\end{array}$ & $\begin{array}{c}Y_{\text {Cit/Glol }} \\
(\mathrm{g} / \mathrm{g})\end{array}$ & $\underset{(\mathrm{Man} / \mathrm{G})}{Y_{\text {Mlol }}}$ & $\begin{array}{c}Y_{\text {Ery/Glol }} \\
(\mathrm{g} / \mathrm{g})\end{array}$ & $\begin{array}{c}\text { IPS } \\
(\mathrm{g} / \mathrm{L})\end{array}$ \\
\hline \multirow{3}{*}{\multicolumn{2}{|c|}{ blank }} & 164 & 10.2 & 2.10 & 67.7 & 8.7 & 11.6 & 8.8 & 0.15 & 20.5 & 0.13 & 0.17 & 0.13 & 0.16 \\
\hline & & 188 & 10.0 & 2.39 & 68.7 & 10.5 & 13.4 & 5.6 & 0.15 & 23.9 & 0.15 & 0.20 & 0.08 & 0.33 \\
\hline & & 260 & 10.8 & 2.33 & 75.5 & 6.2 & $\overline{7.5}$ & 3.7 & 0.14 & 21.6 & 0.08 & 0.10 & 0.05 & 0.88 \\
\hline \multirow{10}{*}{ 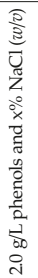 } & \multirow{2}{*}{$0.0 \%$} & 140 & 6.9 & 1.69 & 50.7 & 28.6 & 8.0 & 4.9 & 0.14 & 24.5 & 0.56 & 0.16 & 0.10 & 1.41 \\
\hline & & 188 & 8.7 & 2.47 & 62.8 & 32.7 & 4.5 & 3.7 & 0.14 & 28.3 & 0.52 & 0.07 & 0.06 & 1.52 \\
\hline & \multirow{3}{*}{$1.0 \%$} & 144 & 7.6 & 1.83 & 52.5 & 34.2 & 5.8 & 2.5 & 0.14 & 24.2 & 0.65 & 0.11 & 0.05 & 1.64 \\
\hline & & 224 & 8.1 & 2.07 & 66.3 & 45.2 & 6.0 & 0.0 & 0.12 & 25.7 & 0.68 & 0.09 & 0.00 & 2.59 \\
\hline & & 242 & 8.4 & $\underline{2.52}$ & 69.0 & 21.6 & 5.2 & 0.0 & 0.12 & 29.9 & 0.31 & 0.08 & 0.00 & $\overline{2.40}$ \\
\hline & \multirow[b]{2}{*}{$3.0 \%$} & 140 & 5.6 & 0.91 & 35.8 & 20.8 & 2.9 & 4.9 & 0.16 & 16.2 & 0.58 & 0.08 & 0.14 & 0.46 \\
\hline & & 280 & 4.6 & 1.48 & 53.2 & 42.0 & 3.6 & 0.0 & 0.08 & 31.5 & 0.79 & 0.07 & 0.00 & 0.72 \\
\hline & \multirow{3}{*}{$5.0 \%$} & 210 & 6.5 & 1.63 & 58.3 & 45.7 & 3.1 & 1.9 & 0.12 & 25.2 & 0.88 & 0.06 & 0.04 & 1.72 \\
\hline & & 233 & 6.8 & 2.16 & 66.1 & 54.0 & 1.0 & 0.0 & 0.10 & 31.7 & 0.82 & 0.01 & 0.00 & 0.87 \\
\hline & & 308 & 6.4 & 2.25 & 67.8 & $\overline{51.2}$ & 2.4 & 3.2 & 0.09 & 35.1 & 0.75 & 0.04 & 0.01 & 0.69 \\
\hline
\end{tabular}

In bold we highlight the maximum value of each fermentation compound (i.e. biomass, citric acid, etc) for each trial performed, whereas with bold and underline we highlight the maximum quantity of the given compound between all trials.

The results showed a small reduction of biomass (dry cell weight-DCW; g/L) production and a net reduction of polyols biosynthesis as well as reduced glycerol consumption after the addition of OMWs (viz. phenolic compounds) and $\mathrm{NaCl}$ into the culture medium, while concomitantly, citric acid (Cit) production significantly increased. Potentially, the addition of toxic compounds (i.e. OMWs and $\mathrm{NaCl}$ ) slightly negatively affected biomass production and substrate assimilation, in accordance with several reports in the literature $[3,8,11]$. Moreover, it was observed that the addition of $\mathrm{NaCl}$ combined with the addition of phenolic compounds into the medium favored the yeast to increase the production of lipids in its biomass $\left(Y_{L / X}\right.$ in \% $\left.w / w\right)$. Apparently, the combined effect of the high osmotic pressure and the addition of a "lipogenic" medium, such as the OMWs [3,8], increased the quantity of lipid accumulated inside the yeast cells. In a restricted number of cases, the addition of several types of natural substances (i.e. phenolic compounds, essential oils, etc.) has been revealed to be capable of increasing the content of cellular fatty acids (FAs) inside $Y$. lipolytica yeast [3,8,43], similarly to the current investigation. More specifically, the yeast consumed all glycerol without the presence of OMWs and $\mathrm{NaCl}$ in the culture medium after $260 \mathrm{~h}$ of fermentation, obtaining the highest DCW value between all the experiments (10.8 $\mathrm{g} / \mathrm{L}$ ) with biomass yield $\left(Y_{X / G l o l}\right)=0.14 \mathrm{~g} / \mathrm{g}$ and endopolysaccharides (IPS) $=0.88 \mathrm{~g} / \mathrm{L}$ (corresponding to polysaccharides per DCW value $\left.\left(Y_{I P S / X}\right)=8.1 \% w / w\right)$. However, during this fermentation, maximum production of intracellular lipids, $2.39 \mathrm{~g} / \mathrm{L}$, with maximum lipid in DCW value $\left(Y_{L / X}\right)=23.9 \% w / w$ was observed earlier, at $188 \mathrm{~h}$, at the same time producing the maximum value of citric acid 10.5 $\mathrm{g} / \mathrm{L}$ with $Y_{C i t / G l o l}=0.15 \mathrm{~g} / \mathrm{g}$. At the same conditions (no OMWs and no $\mathrm{NaCl}$ added), the highest values of mannitol $(13.4 \mathrm{~g} / \mathrm{L}$ at $188 \mathrm{~h})$ and erythritol $(8.8 \mathrm{~g} / \mathrm{L}$ at $164 \mathrm{~h})$ were obtained, and these values corresponded to the highest ones obtained in all trials carried out. The addition of OMWs yielding in initial phenolic compounds concentration of $c .2 .0 \mathrm{~g} / \mathrm{L}$ in the culture medium slightly reduced the produced biomass $\left(X_{\max }=8.7 \mathrm{~g} / \mathrm{L}\right)$ and the assimilation rate of glycerol. On the other hand, the amount of the maximum produced intracellular lipids and IPS were slightly increased, while citric acid production was significantly higher compared to the trial with no OMWs added (see Table 1). More specifically, maximum production of polyols $\left(8 \mathrm{~g} / \mathrm{L}\right.$ mannitol with $Y_{\mathrm{Man} / \mathrm{Glol}}=0.16 \mathrm{~g} / \mathrm{g}$ and $4.9 \mathrm{~g} / \mathrm{L}$ erythritol with $Y_{\text {Ery } / G l o l}=0.10 \mathrm{~g} / \mathrm{g}$ ) was observed at $140 \mathrm{~h}$ of fermentation. Maximum production of IPS, $1.52 \mathrm{~g} / \mathrm{L}$ corresponding to $17.5 \%$ of the produced biomass, and $32.7 \mathrm{~g} / \mathrm{L}$ of citric acid with $Y_{\mathrm{Cit} / \mathrm{Glol}}$ $=0.52 \mathrm{~g} / \mathrm{g}$ were obtained at $188 \mathrm{~h}$. At the same time, $Y_{L / X}=28.3 \% w / w$ was obtained. Finally, up to $93 \%$ glycerol consumption was observed. Similar results were observed when $1.0 \% \mathrm{NaCl}$ was added, while the highest values of intracellular lipids and IPS were obtained compared to all fermentations. More specifically, $2.52 \mathrm{~g} / \mathrm{L}$ intracellular lipids were produced at $242 \mathrm{~h}$ with an increased percentage of 
produced lipids per produced biomass $\left(Y_{L / X}=29.9 \% w / w\right)$. The maximum produced IPS was $2.59 \mathrm{~g} / \mathrm{L}$ at $224 \mathrm{~h}$. At the same time, the maximum production of citric acid, $45.2 \mathrm{~g} / \mathrm{L}$, and mannitol, $6.0 \mathrm{~g} / \mathrm{L}$, were obtained, with conversion yields of 0.68 and $0.09 \mathrm{~g} / \mathrm{g}$, respectively. Maximum produced erythritol was just $2.5 \mathrm{~g} / \mathrm{L}$ at $144 \mathrm{~h}$ with $Y_{\text {Ery } / \text { Glol }}=0.05 \mathrm{~g} / \mathrm{g}$.

The increased concentration of $\mathrm{NaCl}$ to $3.0 \%$ in the culture medium resulted in reduced glycerol consumption (up to $76 \%$ ) and decreased maximum DCW and polyols production. Although the absolute values of produced biomass and intracellular lipids were decreased, the maximum lipid in DCW value $Y_{L / X}(\% w / w)$ was increased to $31.5 \%$ at $280 \mathrm{~h}$. Maximum value of DCW, $5.6 \mathrm{~g} / \mathrm{L}$, was observed at $140 \mathrm{~h}$ having, however, the highest produced biomass per consumed substrate between the fermentations $\left(Y_{\mathrm{X} / \mathrm{Glol}}=0.16 \mathrm{~g} / \mathrm{g}\right)$. At the same time, maximum production of erythritol was $4.9 \mathrm{~g} / \mathrm{L}$ with a simultaneous yield $0.14 \mathrm{~g} / \mathrm{g}$. Maximum concentration of IPS was decreased to $0.72 \mathrm{~g} / \mathrm{L}$ at $280 \mathrm{~h}$, corresponding to endopolysaccharides in DCW $=19.8 \% w / w$. Maximum produced citric acid and mannitol were observed at $280 \mathrm{~h}$, with values 42.0 and $3.6 \mathrm{~g} / \mathrm{L}$, respectively, suggesting the positive combined effect of $\mathrm{NaCl}$ and $\mathrm{OMWs}$ addition upon the shift of the cellular metabolism toward citric acid production.

The addition of $5.0 \% w / v$ of $\mathrm{NaCl}$ favored the growth of the yeast, the assimilation of glycerol, and the production of secondary metabolites more than with the addition of $3.0 \% w / v \mathrm{NaCl}$, with the exception of polyols production. At these conditions, the percentage of lipids in produced biomass was further increased, reaching the highest value of $35.1 \% w / w$ with a $2.25 \mathrm{~g} / \mathrm{L}$ maximum of produced intracellular lipid after $308 \mathrm{~h}$ of fermentation. At that time, the maximum glycerol consumption was $90 \% w / w$, and the maximum erythritol production was only $3.2 \mathrm{~g} / \mathrm{L}$. Maximum DCW concentration was $6.8 \mathrm{~g} / \mathrm{L}$, with a yield $Y_{\mathrm{X} / \mathrm{Glol}}=0.10 \mathrm{~g} / \mathrm{g}$ at $233 \mathrm{~h}$, whereas, at the same time, the highest concentration of citric acid ( $54.0 \mathrm{~g} / \mathrm{L}$ with simultaneous yield $Y_{\text {Cit/Glol }}=0.82 \mathrm{~g} / \mathrm{g}$ ) was achieved. Those conditions favored the yeast to produce citric acid in the best conversion yield of citric acid produced per glycerol consumed of all conversions carried out (global conversion yield of citrate produced per unit of glycerol consumed $=0.81 \mathrm{~g} / \mathrm{g}$; see Figure 1 ).

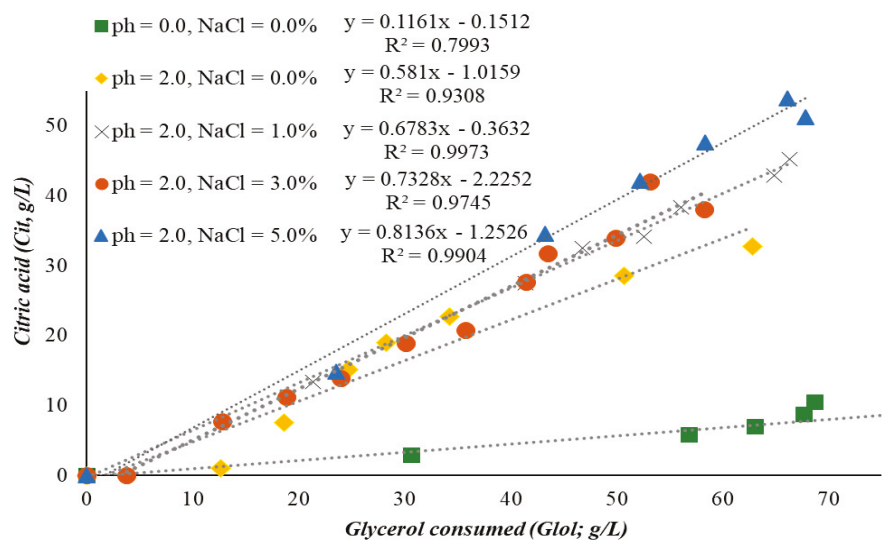

Figure 1. Citric acid production vs glycerol consumption by Yarrowia lipolytica ACA-YC 5031 in $70.0 \mathrm{~g} / \mathrm{L}$ glycerol (blank) and glycerol blended with $2.0 \mathrm{~g} / \mathrm{L}$ phenolic compounds and different concentrations of $\mathrm{NaCl}(0.0 \%, 1.0 \%, 3.0 \%$, and $5.0 \% \mathrm{w} / \mathrm{v})$.

Figure 1 illustrates the correlation between glycerol consumption and citric acid production, and clearly, the more $\mathrm{NaCl}$ is added, the more $Y_{\mathrm{Cit} / \text { /lol }}$ increases. The absence of phenolic compounds and $\mathrm{NaCl}$ from the culture medium (blank experiment) resulted in much less citric acid production per consumed glycerol $(\approx 0.11 \mathrm{~g} / \mathrm{g})$. Citric acid volumetric productivity was $0.05 \mathrm{~g} /(\mathrm{L} \cdot \mathrm{h})$, with a maximum value $10.5 \mathrm{~g} / \mathrm{L}$. While all substrate (glycerol) was consumed with a consumption rate $0.40 \mathrm{~g} /(\mathrm{L} \cdot \mathrm{h})$, 
a significant number of polyols was produced $(20.0 \mathrm{~g} / \mathrm{L})$ at that time, also producing a high amount of biomass (Figure 2). The addition of the phenolic compounds $(2.0 \pm 0.20 \mathrm{~g} / \mathrm{L})$ into the medium resulted in $94 \%$ glycerol consumption, with a consumption rate $0.32 \mathrm{~g} / \mathrm{L} \cdot \mathrm{h})$ and a bit less biomass $\left(X_{\max }=8.7 \mathrm{~g} / \mathrm{L}\right)$ compared to the previous fermentation. A clear decrease in polyols production was also observed, while the productivity of citric acid was faster $(0.18 \mathrm{~g} / \mathrm{L} \cdot \mathrm{h})$, and a significantly increased value, up to $32.7 \mathrm{~g} / \mathrm{L}$, was observed (Figure $2 \mathrm{~b}$ ). The kinetics of glycerol (Glol) consumption, biomass $(X)$ production, polyols biosynthesis ( $\Sigma$ Polyols), and citric acid (Cit) accumulation into the growth medium for all trials performed is shown in Figure 2a-e. Likewise, the illustration of parameters concerning the trials performed is presented in Table 2.
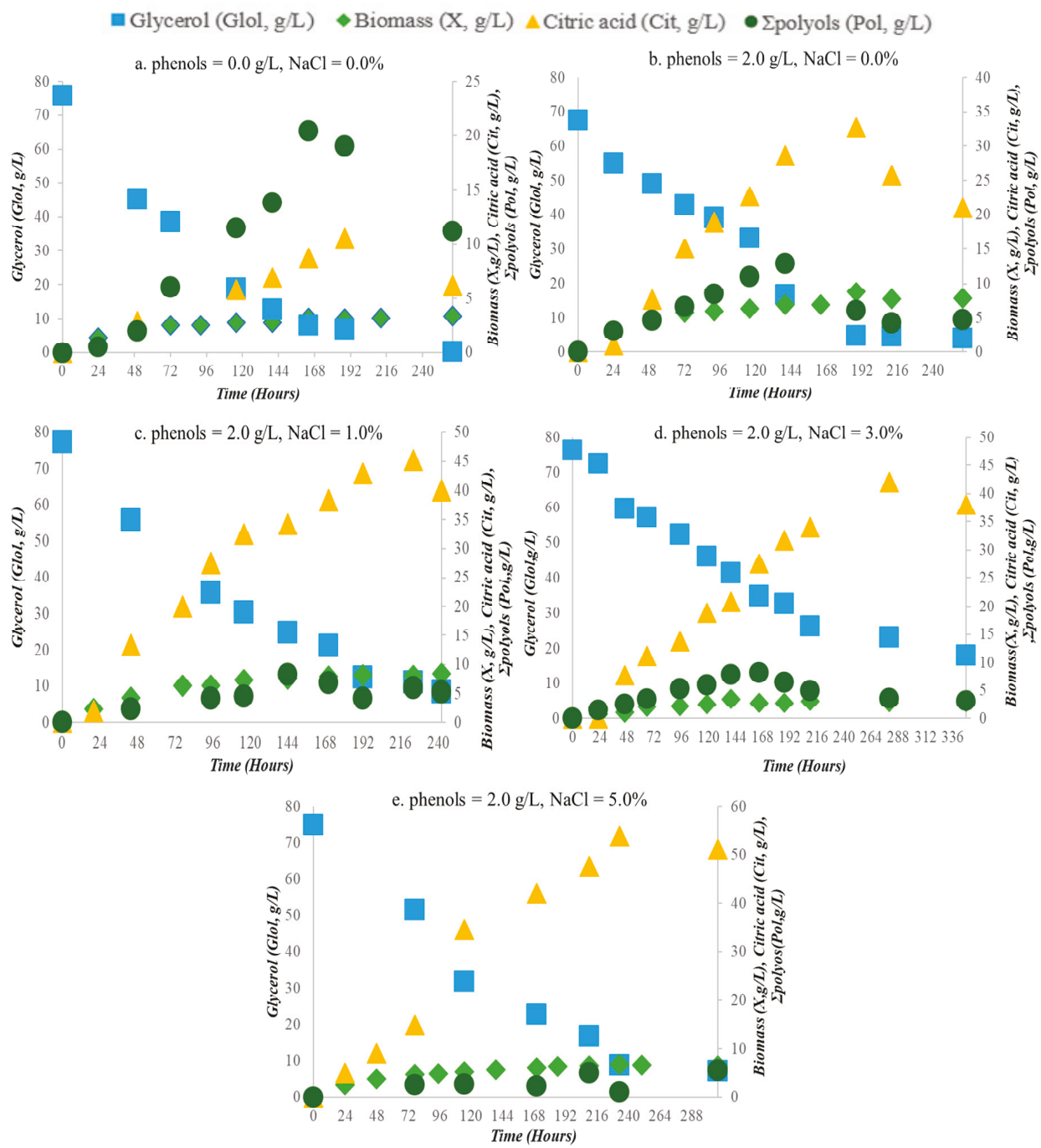

Figure 2. Kinetics of glycerol consumption (g/L), yeast Yarrowia lipolytica ACA-YC 5031 growth (DCW $\mathrm{g} / \mathrm{L})$, citric acid, and polyols production $(\mathrm{g} / \mathrm{L}$ ) in a glycerol-based culture medium containing (a) phenols $=0.0 \mathrm{~g} / \mathrm{L}$ and $\mathrm{NaCl}=0.0 \% ;(\mathbf{b})$ phenols $=2.0 \mathrm{~g} / \mathrm{L}$ and $\mathrm{NaCl}=0.0 \% ;(\mathbf{c})$ phenols $=2.0 \mathrm{~g} / \mathrm{L}$ and NaCl $=$ $1.0 \%$; (d) phenols $=2.0 \mathrm{~g} / \mathrm{L}$ and $\mathrm{NaCl}=3.0 \%$ and $(\mathbf{e})$ phenols $=2.0 \mathrm{~g} / \mathrm{L}$ and $\mathrm{NaCl}=5.0 \%$ concentration. 
Table 2. Glycerol consumption (\%), glycerol consumption rates, citric acid production rates, and specific citric acid production rates during fermentations of glycerol $(70.0 \mathrm{~g} / \mathrm{L})$ with and without phenolic compounds $(2 \mathrm{~g} / \mathrm{L})$ and with increased concentrations of $\mathrm{NaCl}(0.0 \%, 1.0 \%, 3.0 \%$ and $5.0 \% \mathrm{w} / \mathrm{v})$.

\begin{tabular}{cccccc}
\hline Phenolic compounds (g/L) & $\mathbf{0 . 0} \mathrm{g} / \mathrm{L}$ & \multicolumn{4}{c}{$\mathbf{2 . 0} \mathrm{g} / \mathrm{L}$} \\
\hline $\mathrm{NaCl} \%(w / v)$ & $0.0 \%$ & $0.0 \%$ & $1.0 \%$ & $3.0 \%$ & $5.0 \%$ \\
\hline Glycerol consumption $(\% \mathrm{w} / \mathrm{w})$ & $99.6 \%$ & $94 \%$ & $89.1 \%$ & $76.4 \%$ & $90.4 \%$ \\
Glycerol consumption rate, $\mathrm{g} / \mathrm{L} \cdot \mathrm{h})$ & 0.40 & 0.32 & 0.32 & 0.23 & 0.27 \\
Citric acid volumetric productivity, $\mathrm{g} / \mathrm{L} \cdot \mathrm{h})$ & 0.05 & 0.18 & 0.21 & 0.16 & 0.24 \\
Citric acid yield on glycerol consumed, $(\mathrm{g} / \mathrm{g})$ & 0.12 & 0.58 & 0.68 & 0.73 & 0.81 \\
\hline
\end{tabular}

\subsection{Intra-Cellular Lipid Concentration and Fatty Acid Composition}

The intra-cellular lipid concentration was in most of the cases steadily increased during all fermentations (Figure 3). However, the combined effect of the addition of OMWs and $\mathrm{NaCl}$ into the medium increased lipid in DCW values of Y. lipolytica ACA-YC 5031 (without OMWs and NaCl, $Y_{L / X \max }$ value was $23.9 \% \mathrm{w} / \mathrm{w}$, while with the addition of $\mathrm{OMWs}$ and $\mathrm{NaCl}$ at $5 \%$, the respective value was $35.1 \% w / w$-see Table 1). Specifically, the $Y_{L / X \max }$ value increased after the addition of OMW in the culture medium from $23.9 \%$ (blank experiment) to $28.3 \%$ (OMWs added; no $\mathrm{NaCl}$ added). $Y_{L / X \max }$ values continued to increase to $29.9 \%, 31.5 \%$, and $35.1 \%$ with increment of $\mathrm{NaCl}$ concentration in the culture medium $(1.0 \%, 3.0 \%$, and $5.0 \% w / v \mathrm{NaCl}$, respectively) (see Table 1$)$.

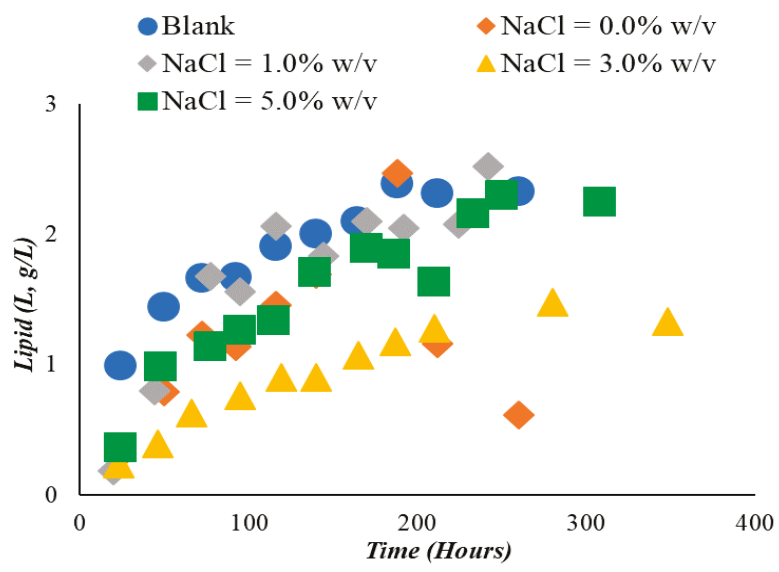

Figure 3. Single cell oil (SCO) production by Yarrowia lipolytica ACA-YC 5031 in culture medium containing blank glycerol and blended also with phenols and $0.0 \%, 1.0 \%, 3.0 \%$, and $5.0 \% \mathrm{w} / \mathrm{v} \mathrm{NaCl}$.

The intracellular fatty acid (FA) composition was analyzed using gas chromatography (GC) and is presented in Table 3. The analyzed fatty acids were palmitic acid, palmitoleic acid, stearic acid, oleic acid, and linoleic acid. The yeast produced a significant amount of C18:1, up to $66.0 \%$, mainly without the presence of phenols and $\mathrm{NaCl}$ in the medium. The addition of phenolic compounds, as well as the increased concentration of $\mathrm{NaCl}$, resulted in a slightly decreased concentration of oleic acid, in contrast to the production of palmitic acid, which it slightly increased from $10.0 \%$ to $16.0 \% \mathrm{w} / \mathrm{w}$. Maximum concentration of palmitoleic was observed when phenols and 3.0\% $w / v \mathrm{NaCl}$ were in the medium (12.0\% and $15.0 \%)$, while a lower concentration of linoleic acid was observed in their absence $(6.0 \%)$. The addition of phenols, as well as of $1.0 \% \mathrm{NaCl}$, resulted in the same concentration of linoleic acid, $11.0 \%$, which was decreased in time. The same phenomenon was observed for $3.0 \%$ and $5.0 \% w / v$ $\mathrm{NaCl}$, reaching $9.0 \%$ and the highest concentration of $21.0 \%$, respectively. Finally, FA C18:0 was found in low concentrations in all trials performed. 
Table 3. Fatty acid composition $(\%, w / w)$ of Yarrowia lipolytica ACA-YC 5031 when grown in a glycerol-based media (70.0 g/L) blended with OMWs $(2.0 \mathrm{~g} / \mathrm{L}$ of phenolic compounds), in which different concentrations of $\mathrm{NaCl}(0.0 \%, 1.0 \%, 3.0 \%$, and $5.0 \% w / v)$ were added.

\begin{tabular}{|c|c|c|c|c|c|c|c|}
\hline pHØ & $\mathrm{NaCl}(\% w / v)$ & Time (h) & C16:0 & C16:1 & C18:0 & C18:1 & C18:2 \\
\hline \multirow{2}{*}{0.0} & \multirow{2}{*}{0.0} & 116 & 10.4 & 10.4 & 6.5 & 66.4 & 6.3 \\
\hline & & 212 & 9.2 & 11.6 & 6.3 & 66.5 & 6.4 \\
\hline \multirow{2}{*}{2.0} & \multirow{2}{*}{0.0} & 50 & 13.0 & 3.5 & 6.6 & 65.3 & 11.6 \\
\hline & & 212 & 11.7 & 10.6 & 6.5 & 62.9 & 8.4 \\
\hline \multirow{2}{*}{2.0} & \multirow{2}{*}{1.0} & 46 & 12.6 & 6.9 & 9.3 & 59.8 & 11.4 \\
\hline & & 116 & 12.3 & 10.9 & 9.1 & 61.6 & 6.1 \\
\hline \multirow{2}{*}{2.0} & \multirow{2}{*}{3.0} & 95 & 13.1 & 12.1 & 8.4 & 57.0 & 9.4 \\
\hline & & 348 & 9.3 & 15.7 & 4.9 & 62.9 & 7.1 \\
\hline \multirow{2}{*}{2.0} & \multirow{2}{*}{5.0} & 48 & 16.0 & 2.9 & 5.2 & 54.7 & 21.1 \\
\hline & & 250 & 10.6 & 11.6 & 4.3 & 59.5 & 14.1 \\
\hline
\end{tabular}

\subsection{Color and Phenolic Compounds Removal}

Y. lipolytica ACA-YC 50231 efficiently removed color and phenolic compounds from the growth medium during most of the trials performed. There was a remarkable $(\approx 40.0 \%)$ color reduction after $50 \mathrm{~h}$ of incubation in the presence of OMWs and $1.0 \% \mathrm{w} / \mathrm{v}$ of $\mathrm{NaCl}$, followed by a $30.0 \%$ decolorization in $3.0 \% \mathrm{NaCl}$ and $17.5 \%$ in $0.0 \% \mathrm{NaCl}$ in the medium. The decolorization became slower following that incubation time. During the other time, only 13.0\% color was removed between 250 and $300 \mathrm{~h}$ of incubation in the presence of a higher concentration $(=5.0 \% \mathrm{w} / \mathrm{v})$ of $\mathrm{NaCl}$ in the medium (Figure 4a). On the other hand, significant removal of phenolic compounds (maximum removal ranging between $52.0-62.0 \% w / w$ irrespective of the quantity of $\mathrm{NaCl}$ initially added into the medium) occurred in all trials performed (Figure $4 b$ ).
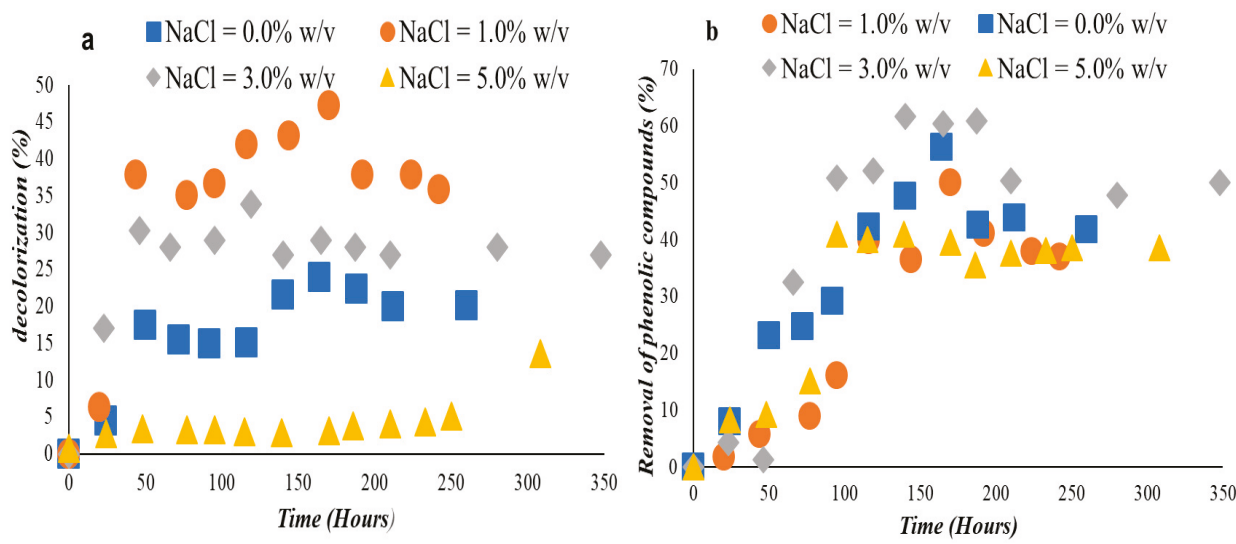

Figure 4. (a) Decolorization (\%) and (b) Removal of phenolic compounds (\%) from the culture medium when Yarrowia lipolytica ACA-5031 was grown in $0.0 \%, 1.0 \%, 3.0 \%$, and $5.0 \% \mathrm{NaCl}$ containing $2.0 \mathrm{~g} / \mathrm{L}$ phenolic compounds.

\section{Discussion}

Shake-flask batch fermentations were carried out to investigate the physiological behavior of the yeast Y. lipolytica ACA-YC 5031 cultivated on biodiesel-derived glycerol employed as the sole carbon source in nitrogen-limited media enriched with OMWs, into which different $\mathrm{NaCl}$ quantities were added. The ability of the yeast to convert crude glycerol into value-added metabolic products 
was examined, as well as its potential to remove phenolic compounds and color from the medium. The obtained results seemed promising since they demonstrated the ability of the yeast to produce value-added compounds under all circumstances, whereas simultaneously remarkable detoxification (decolorization and removal of phenolic compounds) of the employed wastewaters was realized. The amount of the produced secondary metabolites, however, varied significantly between the trials performed, since the addition of $\mathrm{OMWs}$ and $\mathrm{NaCl}$ (in various concentrations) had serious impact upon the physiology of the studied strain. The yeast performed a remarkable growth $(X=10.8 \mathrm{~g} / \mathrm{L}$, $\left.Y_{\mathrm{X} / \mathrm{Glol}} \approx 0.12-0.15 \mathrm{~g} / \mathrm{g}\right)$ in a nitrogen-limited glycerol-based media $\left(\mathrm{Glol}_{0} \approx 70.0 \mathrm{~g} / \mathrm{L}\right.$ ) comparable to previous studies $[20,35,40]$. However, in other cases in which trials of $Y$. lipolytica or other yeasts of Yarrowia clade (i.e. Y. bubula, Y. phangngensis, etc) cultivated in shake-flasks or bioreactor experiments were carried out, significantly higher DCW quantities (i.e. $X>20.0 \mathrm{~g} / \mathrm{L}$, in some cases $X \approx 40.0 \mathrm{~g} / \mathrm{L}$ ) were recorded [54-57].

The presence of OMW (added in initial phenolic compounds $\approx 2.0 \mathrm{~g} / \mathrm{L}$, a typical concentration of phenolic compounds found in OMWs liberated from 3.0- and 2.5-phase centrifuge operation modules [58]) slightly inhibited the cell growth, reducing the DCW to $8.7 \mathrm{~g} / \mathrm{L}$ as previously reported due to the high toxic effect of the effluent $[3,4,58]$. The simultaneous addition of increased salt concentrations resulted in further growth reduction, while the produced intra-cellular polysaccharides did not reach very high values. This phenomenon was reasonable and expected, taking into account that conditions of osmotic stress do not induce cell growth, in contrast to citric acid and polyols production.

Sarris et al. [3] reported a small biomass reduction of related Y. lipolytica strains after the addition of $2.0 \mathrm{~g} / \mathrm{L}$ phenolic compounds in a medium containing $35.0 \mathrm{~g} / \mathrm{L}$ glucose, the principal sugar found in OMWs, and a compound that is metabolized following similar metabolic pathways as those of glycerol. The produced citric acid $(=18 \mathrm{~g} / \mathrm{L})$ and the conversion yield on glucose consumed $(\approx 0.70 \mathrm{~g} / \mathrm{g}$ ) were not affected and remained almost constant. Similarly, Sarris et al. [4] reported a slightly reduced growth of the strain Y. lipolytica ACA-DC 5029 after the addition of OMW in a glycerol-based medium. In that study, the addition of OMW significantly increased citric acid production from $10.5 \mathrm{~g} / \mathrm{L}\left(Y_{\text {Cit } / \mathrm{Glol}}=0.15 \mathrm{~g} / \mathrm{g}\right)$ to $32.7 \mathrm{~g} / \mathrm{L}\left(Y_{\mathrm{Cit} / \mathrm{Glol}}=0.52 \mathrm{~g} / \mathrm{g}\right)$. These results, accompanied with previous studies that reported the successful usage of OMWs in order to dilute concentrated glycerol-based media, suggest that these wastewaters are a suitable medium for citric acid production $[4,58]$. In the present investigation, it was also demonstrated that addition of salt, combined with presence of OMW in the culture medium at ideal $\mathrm{pH}$ conditions $(\mathrm{pH}=5.0-6.0)$, resulted in biosynthesis of citric acid by $Y$. lipolytica at remarkably high quantities. The addition of phenols and the increased concentrations of $\mathrm{NaCl}$ significantly favored the production of citric acid, which it was steadily increased, reaching a maximum quantity of $54.0 \mathrm{~g} / \mathrm{L}$ and a $Y_{\text {Cit/Glol }}$ value $=0.82 \mathrm{~g} / \mathrm{g}$. On the other hand, Tomaszewska et al. [59] observed decreased citric acid production in low $\mathrm{pH}$ conditions $(\mathrm{pH}=3.0)$, while the polyols were significantly increased. In contrast, Rzechonek et al. [60] reported the ability of a genetically modified $Y$. lipolytica to produce a high amount of citric acid, up to $75.8 \mathrm{~g} / \mathrm{L}$, on media containing crude glycerol at $\mathrm{pH}=3.0$. Efficient citric acid production by $Y$. lipolytica strains occurs normally in nitrogen-limited glycerol-based media and $\mathrm{pH}$ values between 5.5 and $7.0[38,40,59]$. The results achieved in the current study ( $\mathrm{Cit}_{\max }=54.0 \mathrm{~g} / \mathrm{L}$ with $Y_{\mathrm{Cit} / \mathrm{Glol}}=0.82 \mathrm{~g} / \mathrm{g}$ ) compares favorably with the ones obtained in shake-flask and batch-bioreactor experiments for both the absolute $(\mathrm{g} / \mathrm{L})$ and relative (g per $\mathrm{g}$ of glycerol consumed) values of produced citric acid. Nevertheless, higher citric acid levels have been reported for conversions carried out in fed-batch bioreactors. A summary of the findings for the conversion of crude glycerol and olive-mill wastewater-based media to citric acid by $Y$. lipolytica strains in various fermentation configurations, including the current study, is given in Table 4.

Erythritol and mannitol were the main byproducts of glycerol metabolism. It is worth noting that traces of arabitol were also observed in the culture medium with and without OMW. However, production of arabitol was not observed in salt containing fermentations, indicating that both the salt and the citric acid inhibited its production, whereas mannitol and erythritol were synthesized at 
low concentrations. More specifically, mannitol and erythritol production reached maximum values $13.4 \mathrm{~g} / \mathrm{L}$ and $8.8 \mathrm{~g} / \mathrm{L}$, respectively, in the glycerol-based medium, while addition of OMW reduced their production. Rymowicz et al. [26] and Chatzifragkou et al. [42] reported that it is essential for polyol biosynthesis an appropriate high initial glycerol concentration. Although high glycerol concentration favored the production of mannitol, Rywińska et al. [34] reported higher erythritol production than mannitol in a crude glycerol substrate. Moreover, it has been found that growth of osmophilic yeasts in glycerol-based medium containing salt at low $\mathrm{pH}$ values promoted erythritol production $[23,39,40]$, a fact that was not validated in the current investigation since, as it has been previously reported, the addition of salt in OMW/glycerol blends clearly shifted the cellular metabolism towards citric acid production instead of polyols (see Figure $2 \mathrm{a}-\mathrm{e}$ ).

Table 4. Metrics of citric acid production from glycerol- or OMW-based media by Yarrowia lipolytica strains cultivated on various fermentation configurations.

\begin{tabular}{|c|c|c|c|c|c|}
\hline Strain & $\begin{array}{c}\text { Citric acid } \\
(\mathrm{g} / \mathrm{L})\end{array}$ & Substrate & $\begin{array}{l}\text { Yield } \\
(\mathrm{g} / \mathrm{g})\end{array}$ & Fermentation Type & Reference \\
\hline ACA-DC 50109 & 33.6 & Crude glycerol & 0.44 & Shake flasks & {$[38]$} \\
\hline Wratislavia 1.31 & 124.5 & $*_{\gg}$ & 0.62 & Batch bioreactor & [26] \\
\hline Wratislavia AWG7 & 88.1 & $»$ & 0.46 & » & 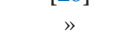 \\
\hline Wratislavia K1 & 75.7 & $»$ & 0.40 & $»$ & $»$ \\
\hline ACA-DC 50109 & 28.9 & Glucose/OMWs & 0.53 & Shake flasks & [11] \\
\hline$»$ & 62.5 & Crude glycerol & 0.56 & $»$ & [61] \\
\hline A-101-1.22 & 112.0 & $»$ & 0.60 & Batch bioreactor & [27] \\
\hline ACA-YC 5033 & 50.1 & $»$ & 0.44 & Shake flasks & [20] \\
\hline A-101 & 66.5 & Pure glycerol & 0.44 & Batch bioreactor & [30] \\
\hline$»$ & 66.8 & Crude glycerol & 0.43 & $»$ & $»$ \\
\hline Wratislavia K1 & 53.3 & Pure glycerol & 0.34 & $»$ & $»$ \\
\hline$»$ & 36.8 & Crude glycerol & 0.25 & $»$ & $»$ \\
\hline Wratislavia 1.31 & 126.0 & $»$ & 0.63 & Fed-batch bioreactor & [31] \\
\hline Wratislavia AWG7 & 157.5 & $»$ & 0.58 & $»$ & $》$ \\
\hline Wratislavia 1.31 & 155.2 & $»$ & 0.55 & $»$ & $»$ \\
\hline Wratislavia AWG7 & 154.0 & $»$ & 0.78 & Repeated batch & [29] \\
\hline N15 & 19.08 & Pure glycerol & 0.55 & Shake flasks & [32] \\
\hline$»$ & 98.0 & 》 & 0.70 & Fed-batch bioreactor & 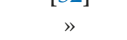 \\
\hline Wratislavia AWG7 & 86.5 & $»$ & 0.59 & Continuous bioreactor ${ }^{a}$ & [41] \\
\hline » & 63.3 & $»$ & 0.67 & Continuous bioreactor ${ }^{b}$ & $»$ \\
\hline W29 & 15.8 & Glucose/OMWs & 0.46 & Shake flasks & {$[62]$} \\
\hline ACA-YC 5033 & 18.1 & $»$ & 0.51 & » & 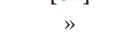 \\
\hline NG40/UV7 & 115.0 & Pure glycerol & 0.64 & Fed-batch bioreactor & [44] \\
\hline$»$ & 112.0 & Crude glycerol & 0.90 & 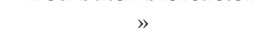 & $»$ \\
\hline JMY1203 & 57.7 & $》$ & 0.92 & Shake flasks & [28] \\
\hline ACA-YC 5029 & 39.0 & $»$ & 0.42 & Batch-bioreactor & [63] \\
\hline ACA-YC 5033 & 15.2 & Glucose/OMWs & 0.61 & Batch-bioreactor & [3] \\
\hline$»$ & 13.9 & $»$ & 0.58 & Batch-bioreactor $^{\mathrm{c}}$ & $"$ \\
\hline$»$ & 51.9 & $»$ & 0.64 & Shake flasks & $»$ \\
\hline ACA-DC 5029 & 37.4 & Glycerol/OMWs & 0.55 & $»$ & [4] \\
\hline$»$ & 79.0 & Crude glycerol & 0.46 & $»$ & $»$ \\
\hline ACA-YC 5031 & $54.0^{\mathrm{d}}$ & Glycerol/OMWs & 0.82 & Shake flasks & $\begin{array}{c}\text { Present } \\
\text { study }\end{array}$ \\
\hline
\end{tabular}

* „: same as above; ${ }^{\mathrm{a}}: D=0.009 \mathrm{~h}^{-1} ; \mathrm{b}: D=0.021 \mathrm{~h}^{-1} ;{ }^{\mathrm{c}}$ : Trial under non-aseptic conditions; ${ }^{\mathrm{d}}$ : Total citric acid, sum of citric and iso-citric acid;

Although the biochemical events leading to the synthesis of polyols from glycerol in $Y$. lipolytica strains have not been completely elucidated [20,35,42], it appears that the biosynthesis of polyols depends on the response of the enzyme complexes to the external osmotic environment. More specifically, high osmotic pressure increases the activity of the enzyme erythrose reductase (EP), while it reduces the activity of the enzyme mannitol-1-phosphate dehydrogenase (M-1-PDH) [23]. In 
this study, under all circumstances where salt was added, mannitol production was observed after 24 $h$ incubation until the end of the fermentations, while erythritol production was observed later and for a very short period. Since erythritol molecule with a smaller MW than the mannitol, the osmotic pressure that comes from erythritol is higher than that of mannitol at the same concentrations of both compounds. When the microorganism is exposed to a high osmotic pressure, it produces erythritol to balance the osmotic pressure extra-cellularly and intra-cellularly. The organism accumulates a high amount of erythritol, which compensates for the difference between the extracellular and intracellular water potential [27]. Other parameters that affect erythritol production are the $\mathrm{pH}$ of the culture medium (production of polyols is mainly performed at $\mathrm{pH}$ ranging between 2.5 and 3.5), the incubation temperature, the type and the concentration of the carbon source employed, the nitrogen and phosphate source, as well as the presence of some metals [59]. On the other hand, many studies have reported mannitol to be the only polyol produced by the yeast in order to be protected against the osmotic stress. Without doubt, the cooperative activity between those two polyols can protect the cell from damages by that kind of stress [23]. In general, the production of polyols from glycerol is not common for $Y$. lipolytica strains and can be very interesting for Food Technology.

Y. lipolytica is a well-known lipid-producing microorganism employed in the literature for the production of common and non-common fatty acids due to its ability to accumulate over $20.0 \%$ and up to $70.0 \%$ lipids per dry weight $(w / w)$ [51,64]. Dobrowolski et al. [65] have reported lipid content $25 \%$ of DCW in Y. lipolytica A101 on media containing crude glycerol from soap production, similar to this study on the glycerol-based medium. Lipid production by Y. lipolytica ACA YC-5031 increased from $23.9 \%$ of DCW on glycerol-based medium to $28.3 \%$ after the addition of phenolic compounds into the glycerol-based medium. Similarly, Sarris et al. [3,4] reported a proportionally increased in SCO production after the addition of OMWs in the culture medium. These results confirm the potential usage of OMW as a "lipogenic" substrate $[3,58]$. The addition of salt combined with OMW in the culture medium favored the production of intra-cellular lipids, which increased with increased salt concentration, reaching $Y_{L / X \max }=35.1 \% w / w$ when $5.0 \% w / v \mathrm{NaCl}$ had been added.

Biosynthesis of both citric acid and SCO from carbon sources like glycerol or similarly metabolized compounds in Y. lipolytica are processes that present remarkable similarities in their first steps being triggered by the depletion of an essential nutriment, and in particular nitrogen, from the culture medium $[37,51,61,64,66-68]$. Upon nitrogen limitation and in the presence of excess carbon, Y. lipolytica yeast strains produce large amounts of TCA cycle intermediates, including citric acid and iso-citric acid, which are not further catabolized. In essence, nitrogen exhaustion results in a rapid decrease of the intra-cellular AMP (adenosine monophosphate) concentration, as AMP is cleaved to produce $\mathrm{NH}_{4}{ }^{+}$ions, indispensable for cell growth. This event results in the inactivation of iso-citrate dehydrogenase in the TCA cycle and, consequently, the accumulation of both iso-citric and citric acids into the mitochondria. When the intra-mitochondrial citric acid concentration reaches a critical value, citrate enters the cytoplasm in exchange for malate. Citric acid is then either cleaved by ATP-citrate lyase (ACL), the key enzyme of the lipid accumulation process in oleaginous microorganisms, to yield acetyl-CoA and oxaloacetate, or it is secreted to the culture medium $[37,66,68]$. For the case of lipid-accumulating microorganisms, the resulting acetyl-CoA is carboxylated by acetyl-CoA carboxylase (ACC1) to form malonyl-CoA, the substrate for the biosynthesis of acyl-CoA esters and, subsequently, triacylglycerols $[37,66,68]$.

Y. lipolytica strains are a most untypical example of the group of oleaginous yeasts [68]. Lipid content of various strains during growth on glucose under nitrogen limitation (condition favoring lipid storage) is not very high in several cases (e.g. biomass in $\mathrm{DCW} \approx 36 \% w / w)$. It certainly does not compare favorably for lipid accumulation with species of Rhodotorula/Rhodosporidium, Lipomyces, and other genera $[66,68]$. In some cases observed during growth in a shake-flask or batch bioreactor experiments, growth of $Y$. lipolytica in nitrogen-limited glucose- or glycerol-based media resulted in sequential production of intra-cellular lipid and extra-cellular citric acid. In the first steps of nitrogen limitation, lipid accumulation was triggered (the maximum lipid in DCW was rarely $>25 \%$, $w / w$ ), 
and thereafter, lipid content decreased with time, even though significant substrate quantities remained unconsumed in the medium. The period of intra-cellular lipid degradation (turnover) coincided with the secretion of citric acid in non-negligible quantities into the culture medium $[25,28,35,53,63,67]$. On the other hand, in other studies, as in the current investigation, lipid content as a proportion of the DCW constantly increased over the whole range of the nitrogen-limited batch fermentation, with simultaneous significant citric acid secretion $[62,65]$. The above-mentioned complex regulation makes it difficult in many instances to obtain high rates of lipid accumulation in batch culture, since, in such conditions, lipid accumulation and citric acid production occur simultaneously, resulting in only moderate lipid accumulation, as in the current investigation. Potentially, this fact was the reason for which, in the first reports that have appeared, the ability of yeasts of the species Y. lipolytica to produce lipids from glucose, glycerol, or similarly metabolized compounds has been characterized as "dubious" [53,66,68].

In the trials performed by Y. lipolytica ACA-YC 5031, the produced fatty acids (FAs) were palmitic (C16:0), palmitoleic (C16:1), stearic (C18:0), oleic (C18:1), and linoleic (C18:2) acid. Oleic acid being the dominant fatty acid under all circumstances, is in agreement with previous reports $[4,20,25,27,42,58,62]$, indicating that SCO produced by Y. lipolytica ACA YC-5031 through the type of the conversion proposed, can constitute perfect precursors for the synthesis of second-generation biodiesel $[20,50]$. Fatty acid desaturase activity during cultivation was estimated by calculating the ratios of desaturase product to substrate (C16:1/C16:0; C18:1/C18:0; C18:2/C18:1). High C18:1/C18:0 ratios can be observed, in accordance with previous studies carried out by several wild-type mutant $Y$. lipolytica strains tested $[4,20,27,42,43]$, indicating an important $\Delta 9$-desaturase activity in the yeast cells, as reported by Rymowicz et al. [27] and Sarris et al. [4]. Sarris et al. [62] found that the addition of OMW in the culture medium increased the amount of oleic acid over $60.0 \%$ in time, while it reduced that of C18:0. However, in this study, the addition of OMW slightly reduced the amount of oleic acid, while the addition of increased salt concentration resulted in a slightly further reduction of the above-mentioned cellular FA (see Table 3). Nevertheless, cellular C18:0 concentrations were always $\approx 60.0 \% w / w$, showing that addition of salt to the OMW/glycerol blends affected to the minimum the FA composition of the cellular lipids produced (Table 3).

In all trials performed, and irrespective of the addition of $\mathrm{NaCl}$ into the OMW-based media employed, Y. lipolytica ACA-YC 5031 performed a significant removal of phenolic compounds into the medium (between $40.0-62.0 \% w / w$ ), while a non-negligible decolorization was also observed (Figure $4 \mathrm{a}, \mathrm{b}$ ). At the highest $\mathrm{NaCl}$ concentrations employed, a lower decolorization rate was observed, but, in any case and in accordance with the literature [3,7,8,11,62], the removal of phenolic compounds and the removal of color from the fermented OMW-based media was not proportional, meaning, in fact, that high decolorization did not mean that simultaneously high removal of phenolic compounds occurred, and vice versa. Sarris et al. [3] reported 55.9\% decolorization and simultaneous $12.9 \%$ removal of phenolic compounds when somehow low initial concentrations of phenolic compounds were employed $(\approx 1.80 \mathrm{~g} / \mathrm{L})$, while the respective values of removal of phenolic compounds and color were $50.9 \% w / w$ and $45.3 \%$ when OMW-based media with initial phenolic compounds $\approx 5.50 \mathrm{~g} / \mathrm{L}$ were employed for the related strain Y. lipolytica ACA-YC 5033 in OMW and glucose-enriched media. Similarly, the yeast Y. lipolytica ACA-YC 5029, as reported by Sarris et al. [4], performed up to $10.0 \% w / w$ phenols removal and up to $30.0 \%$ color removal during growth on crude glycerol. In general, the ability of some yeasts and fugal strains to remove phenolic compounds depends on the secretion of extracellular oxidases, laccases, and other enzymes, such as lignin peroxidases and manganese-dependent (or independent) peroxidases $[4,8,11]$. However, the yeasts do not have the appropriate mechanisms to produce those enzymes and to remove the phenolic compounds. Therefore, the phenol and color reduction might be attributed to potential adsorption of phenolic compounds in the yeast cells or even to their partial utilization as carbon source and energy [4]. 


\section{Materials and Methods}

\subsection{Microorganism, Media, and Culture Conditions}

The yeast Y. lipolytica ACA-YC 5031 isolated from various types of sourdoughs [69] was kindly offered by the Laboratory of Dairy Science, Agricultural University of Athens, Greece. The organism was maintained on YPDA slants (10 g/L glucose, $10 \mathrm{~g} / \mathrm{L}$ yeast extract, $10 \mathrm{~g} / \mathrm{L}$ peptone, and $20 \mathrm{~g} / \mathrm{L}$ agar) at $4^{\circ} \mathrm{C}$. The experiments were carried out in 250-mL Erlenmeyer flasks, containing $50 \pm 1 \mathrm{~mL}$ of growth medium, sterilized at $T=121^{\circ} \mathrm{C}$ for $20 \mathrm{~min}$ and inoculated with $1 \mathrm{~mL}$ of 24 -h exponential pre-culture yeast incubated at $3.0 \mathrm{~Hz}$ at $28 \pm 1{ }^{\circ} \mathrm{C}$. The yeast pre-culture was carried out in yeast extract-glucose-dextrose medium with $10.0 \mathrm{~g} / \mathrm{L}$ of each. Biodiesel-derived glycerol (initial glycerol concentration- $-\mathrm{Glol}_{0}=70 \pm 5 \mathrm{~g} / \mathrm{L}$ ) was used as a carbon source in the medium, while peptone and yeast extract, $1.0 \mathrm{~g} / \mathrm{L}$ each, were used as a nitrogen source. Two types of fermentation were examined a) containing OMWs that were added in order to yield in initial concentration $2.0 \pm 0.20 \mathrm{~g} / \mathrm{L}$ of phenolic compounds and b) without OMW in the culture medium. The origin and the composition of crude glycerol and the chemical composition of OMWs employed in the current investigation were as in Sarris et al. [4]. Specifically, OMWs were received from a three-phase decanter olive mill located in Chania (Crete, Greece) and were frozen at $T=-20^{\circ} \mathrm{C}$. Prior to the experiments, OMWs were de-frozen, and the solids were removed by centrifugation $\left(9000 \times \mathrm{g} / 15 \mathrm{~min}\right.$ at $\left.T=21^{\circ} \mathrm{C}\right)$ in a Universal 320R-Hettich centrifuge (Tuttlingen, Germany). OMW phenolic content expressed as gallic acid equivalent was $\sim 3.5 \mathrm{~g} / \mathrm{L}$, while no reducing sugars were found into the wastewaters. Moreover, negligible quantities of olive oil (c. $0.2 \mathrm{~g} / \mathrm{L}$; determination of oil conducted after triple extraction with hexane) were presented into the OMWs tested. Therefore, in the trials performed, the sole carbon source employed was crude glycerol. The culture medium salt composition was (g/L): $\mathrm{KH}_{2} \mathrm{PO}_{4}, 7.0$; $\mathrm{Na}_{2} \mathrm{HPO}_{4} \times 2 \mathrm{H}_{2} \mathrm{O}, 2.5 ; \mathrm{MgSO}_{4} \times 7 \mathrm{H}_{2} \mathrm{O}, 1.5 ; \mathrm{MnSO}_{4} \times \mathrm{H}_{2} \mathrm{O}, 0.06 ; \mathrm{ZnSO}_{4} \times 7 \mathrm{H}_{2} \mathrm{O}, 0.02 ; \mathrm{FeCl}_{3} \times 6 \mathrm{H}_{2} \mathrm{O}, 0.15$; $\mathrm{CaCl}_{2} \times 2 \mathrm{H}_{2} \mathrm{O}, 0.15$ [38]. As a nitrogen source, peptone and yeast extract were used in a concentration of $1.0 \mathrm{~g} / \mathrm{L}$ each, imposing nitrogen-limited conditions in all trials. Experiments were carried out evaluating the effect of $\mathrm{NaCl}$ at different concentrations $(0.0 \%, 1.0 \%, 3.0 \%$, and $5.0 \% w / v)$ in the culture medium. All fermentations carried out in shake-flask mode, and flasks were placed in an orbital shaker (New Brunswick Sc, USA) at an agitation rate of $3.0 \mathrm{~Hz}\left(\mathrm{~T}=28 \pm 1^{\circ} \mathrm{C}\right)$. Initial $\mathrm{pH}$ in the culture media was adjusted to 6.0 while $\mathrm{pH}$ value during the trials was measured and when necessary it was adjusted between the range $4.8-5.8$ by adding (periodically and aseptically) small quantities $\mathrm{NaOH}$ $5 \mathrm{M}$ (e.g. 500-600 $\mu \mathrm{l}$ ) [3,11]. The exact volume of $\mathrm{NaOH}$ solution needed for the $\mathrm{pH}$ correction was evaluated by measuring the volume of $\mathrm{NaOH}$ solution required for $\mathrm{pH}$ correction in one (at least) flask (collected daily). Then the appropriate volume of $\mathrm{NaOH}$ solution was aseptically added in the remaining flasks and the value of $\mathrm{pH}$ reached was verified to be in the range of 4.8-5.8.

\subsection{Dry Weight Determination}

The whole content of the $250 \mathrm{~mL}$ flasks was collected, and cells were harvested by centrifugation at $9000 \times \mathrm{g} / 10 \mathrm{~min}$ at $\mathrm{T}=4{ }^{\circ} \mathrm{C}$ using a Universal 320R-Hettich centrifuge (Tuttlingen, Germany). The pellet was then washed with distilled water, and centrifugation was applied one more time under the same conditions. Biomass $(X)$ was dried at $T=90 \pm 5^{\circ} \mathrm{C}$ for 24 hours to obtain the dry cell weight (DCW) expressed in $\mathrm{g} / \mathrm{L}$ [38]. Biomass yield $Y_{X / G l o l}(\mathrm{~g} / \mathrm{g})$, was expressed as the grams of cell dry weight $(X)$ produced, per grams of substrate (glycerol; Glol) consumed (g DCW / g glycerol consumed).

\subsection{Determination of Total Intra-Cellular Polysaccharides (IPS)}

Determination of IPS was carried out using a modified protocol described by Liang et al. [70] and the 3,5-dinitrosalicylic acid method (DNS) described by Miller [71]. Specifically, $0.05 \mathrm{~g}$ DCW were acidified by adding $10 \mathrm{~mL} \mathrm{HCl} 2 \mathrm{M}$. The solution was then hydrolyzed at $T=100{ }^{\circ} \mathrm{C}$ for $30 \mathrm{~min}$, followed by addition of $10 \mathrm{~mL} \mathrm{NaOH} 2 \mathrm{M}$ to reach $\mathrm{pH}=7.0$, and was subsequently filtered through Whatman filter paper twice. Then, $0.5 \mathrm{~mL}$ of the sample solution and $0.5 \mathrm{~mL}$ DNS reagent were 
transferred into tubes and left in a water-bath at $\mathrm{T}=100^{\circ} \mathrm{C}$ for $5 \mathrm{~min}$, followed by $2 \mathrm{~min}$ a $\mathrm{T}=25^{\circ} \mathrm{C}$. Finally, $5 \mathrm{~mL}$ distilled water were added to the samples mixing well, and the absorbance at $540 \mathrm{~nm}$ was measured using a Hitachi U-2000 Spectrophotometer (Tokyo, Japan).

\subsection{Determination of Glycerol, Polyols, and Citric Acid}

The concentration of the remaining glycerol, the produced polyols, and citric acid were determined during the fermentation using Waters 600E High-performance liquid chromatography (HPLC), (Waters Association, Milford, MA, USA) [72]. The samples were first filtered using a membrane of $0.2 \mu \mathrm{m}$ diameter, and $10 \mu \mathrm{l}$ of the sample were injected. The mobile phase was $\mathrm{H}_{2} \mathrm{SO}_{4} 5 \mathrm{mM}$, while the static phase was the column Amimex HPX-87H (Biorad, Richmond, CA, USA) (30 cm x $7.8 \mathrm{~mm}$ ). The column flow was $0.5 \mathrm{~mL} / \mathrm{min}$, at $T=45^{\circ} \mathrm{C}$. The apparatus type was Waters $600 \mathrm{E}$ with RI detector (RI; Waters 410) for the determination of glycerol and polyols and UV detector (Waters 486) for the determination of organic acids. The area of each compound was determined according its retention time, and the concentration of each compound (glycerol, citric acid, mannitol, erythritol) was determined using reference curves and expressed as $\mathrm{g} / \mathrm{L}$. Citric and iso-citric acid were not totally separated with the implicated HPLC analysis method, and the reported concentration corresponds to the sum of these acids, expressed as total citric acid (Cit). In order to proceed with a more precise determination of iso-citric acid, in some of the fermentation points, an enzymatic method, based on the measurement of the $\mathrm{NADPH}_{2}$ produced during conversion of the iso-citric to $\alpha$-ketoglutaric acid, the reaction catalyzed by the iso-citrate dehydrogenase, was employed. In all points where iso-citrate determination occurred, iso-citric acid represented a quantity of $5-7 \%(w / w)$ of total citric acid produced, regardless of the culture conditions employed.

Glycerol assimilation rate (in $\mathrm{g} / \mathrm{L} \cdot \mathrm{h}$ ) was expressed as substrate removed $(\mathrm{g}$ ) per $\mathrm{L}$ of medium and per hour $(=-\Delta \mathrm{Glol} / \Delta \mathrm{t})$ for the respective time in which fermentation was performed.

Total citric acid yield, $Y_{\text {Cit/Glol }}(\mathrm{g} / \mathrm{g})$, was calculated based on the grams of produced total citric acid (Cit) per grams of substrate consumed (Glol).

Citric acid volumetric productivity (in $\mathrm{g} / \mathrm{L} \cdot \mathrm{h}$ ) was determined as the concentration of citric acid in a given fermentation time divided by this respective time.

The global yield of the produced citric acid per glycerol consumed $\left(Y_{\text {Cit/Glol }}\right)$ was determined as the produced citric acid in a plot against the consumed substrate [Cit $=f$ (Glol consumed)] with equation $\mathrm{y}=a \mathrm{x}+\mathrm{b}$, where " $a$ " represents the citric acid yield $Y_{\mathrm{Cit} / \mathrm{Glol}}$.

Mannitol yield $Y_{M a n / G l o l}(\mathrm{~g} / \mathrm{g})$, was calculated based on the grams of produced mannitol (Man) per grams of substrate consumed (Glol).

Erythritol yield, $Y_{\text {Ery/Glol }}(\mathrm{g} / \mathrm{g})$, was calculated based on the grams of produced erythritol (Ery) per grams of substrate consumed (Glol).

\subsection{Quantitative Determination of the Cellular Lipid and Fatty Acid (FA) Composition Analysis}

Total cellular lipid was extracted from DCW with a chloroform-methanol mixture (30 mL, 2:1, $\mathrm{v} / \mathrm{v})$ after $72 \mathrm{~h}$ in the darkness [38]. After three days, cell debris were removed through filtration (Whatman $囚$ filter $n^{\circ} 3$ ) and the solvent mixture was completely evaporated in a rotary evaporator (R-144, Büchi Labortechnik, Flawil, Switzerland) at $T=60-65^{\circ} \mathrm{C}$. Total intra-cellular lipid, L, was determined gravimetrically and was expressed as g/L. Lipid in DCW $\left(\%, w / w ; Y_{L / X}\right)$ was calculated based on percentage of the accumulated lipid $(L)$ per produced dry biomass $(X)$.

The extracted intra-cellular lipids were converted to their fatty acid methyl-esters (FAMEs) and analyzed in a gas chromatography (GC) (Fisons 8000 series, Offenbach, Germany) equipped with an FID (Fisons) according to the method described by Zikou et al. [73]. Methyl-esterification was performed in two phases. One to two boiling stones and $10 \mathrm{~mL}$ sodium methoxide $\left(\mathrm{MeO}^{-} \mathrm{Na}^{+}\right)$were added into the extracted total lipids. The samples were left to boil for $20 \mathrm{~min}$. Methanol hydrochloride $\left(\mathrm{CH}_{3} \mathrm{OH}-\mathrm{HCl}\right)$ was then added until the decolorization of the mixture, which reached a milky color. Boiling was then continued for another $20 \mathrm{~min}$ before the addition of distilled water to end the reaction. 
Finally, $6 \mathrm{~mL}$ of hexane were added, and the mixture was shaken vigorously. The solvent phase was collected, and a $1 \mu \mathrm{l}$ sample was injected into GC.

\subsection{Phenolic Compounds Determination}

Determination of total phenol compounds $(\mathrm{pH}$ ) into the medium was carried out according to the method described by Aggelis et al. [8]. Sampleof $0.2 \mathrm{~mL}$ was mixed with $10.8 \mathrm{~mL}$ distilled water, $8 \mathrm{~mL} \mathrm{Na}_{2} \mathrm{CO}_{3}(75 \mathrm{~g} / \mathrm{L})$, and $1 \mathrm{~mL}$ Folin-Ciocalteu reagent. Blank samples used as controls were prepared using $0.2 \mathrm{~mL}$ of distilled water. The mixture was shaken and remained in darkness for $2 \mathrm{~h}$. The absorbance was measured at $750 \mathrm{~nm}$ in a Hitachi U-2000 Spectrophotometer (Tokyo, Japan). The concentration of phenolic compounds was expressed in equivalence of gallic acid according to a reference curve.

\subsection{Decolorization}

In order to determine the decolorization efficiency (color removal) of the fermentations, a $0.5 \mathrm{~mL}$ sample was mixed well with $14.5 \mathrm{~mL}$ distilled water, and the absorbance was measured at $395 \mathrm{~nm}$ according to Sayadi and Ellouz [74]. The decolorization percentage was calculated using the equation $\% \mathrm{~A}=\left[\left(\mathrm{A}_{0}-\mathrm{A}_{1}\right) / \mathrm{A}_{0}\right] \times 100$, where, $\mathrm{A}_{0}$ is the absorbance at time 0 and $\mathrm{A}_{1}$ is the absorbance at each experimental point during the fermentation.

\subsection{Determination of Extra-Cellular Nitrogen Into the Fermentation Medium}

Extra-cellular non-assimilated nitrogen was measured in some of the fermentation points of the trials performed according to free amino nitrogen (FAN) determination. FAN concentration into the liquid samples was determined according to Kachrimanidou et al. [72].

\subsection{Data Analysis}

All experiments and analyses were performed in duplicate. Each experimental point of all of the kinetics presented in the tables and figures is the mean value of two independent determinations. Data were plotted using Microsoft Office Excel Version 2007 showing the mean values with the standard error mean. Throughout the text, indices 0 and max represent the initial and the maximum quantity of the elements in each kinetics presented.

\section{Conclusions}

The addition of increased concentrations of $\mathrm{NaCl}$ to OMW/glycerol blends, a novel application, showed an interesting physiological response of the yeast $Y$. lipolytica. In essence, the combined effect of the addition of OMWs and $\mathrm{NaCl}$ into the medium increased lipid in DCW values of Y. lipolytica ACA-YC 5031, while remarkable biomass production was observed in all trials. Although glycerol metabolism, in the experiment with no OMWs and no salt added, was mainly shifted toward the synthesis of polyols, the addition of OMWs resulted in a reduction of $\Sigma$ polyols concentration and a significant increase in the production of citric acid. The addition of salt in the OMW-based media resulted in a drastic increase in citric acid production. reaching maximum values in the trial with the addition of $5.0 \% \mathrm{NaCl}$. These results indicate that the yeast $Y$. lipolytica is a great candidate for the production of value-added compounds. The results showed that the addition of $\mathrm{NaCl}$ positively affected the physiological behavior of the yeast, having a great impact on the production of citric acid and lipids by this microorganism. Additionally, OMWs were found to be a promising substrate for the production of citric acid, mainly combined with salt. Finally, simultaneous with the production of several added-value metabolites, the non-negligible color and phenolic compounds removal from the medium with the aid of Y. lipolytica suggested that the yeast can also be used as an important microbial cell factory amenable for the depollution and detoxification of several phenol-rich recalcitrant wastewaters that are frequently generated during various agro-industrial activities. 
Author Contributions: Conceptualization, S.P.; Methodology, M.K., S.P. and D.S; Validation, M.K and S.P.; Formal Analysis, M.T, M.K. and S.P.; Investigation, M.K.; Resources, A.A.K. and S.P.; Writing - Original Draft Preparation, M.T. and S.P.; Writing - Review \& Editing, M.T. and S.P.; Supervision, D.S., A.A.K. and S.P.; Project Administration, A.A.K. and S.P; Funding acquisition, S.P.

Funding: This research was funded by the project entitled "Adding value to biodiesel-derived crude glycerol with the use of Chemical and Microbial Technology" (Acronym: Addvalue2glycerol; grant number T1E $\Delta$ K-03002), which is financially supported by the Ministry of National Education and Religious Affairs, Greece (project action: "Investigate - Create - Innovate 2014-2020, Intervention II").

Conflicts of Interest: The authors declare no conflict of interest.

\section{References}

1. Mantzavinos, D.; Kalogerakis, N. Treatment of olive mill effluents, Part I. Organic matter degradation by chemical and biological processes-an overview. Environ. Int. 2005, 31, 289-295. [CrossRef] [PubMed]

2. Crognale, S.; D’Annibale, A.; Federici, F.; Fenice, M.; Quaratino, D.; Petruccioli, M. Olive oil mill wastewater valorization by fungi. J. Chem. Technol. Biotechnol. 2006, 81, 1547-1555. [CrossRef]

3. Sarris, D.; Stoforos, N.G.; Mallouchos, A.; Kookos, I.K.; Koutinas, A.A.; Aggelis, G.; Papanikolaou, S. Production of added-value metabolites by Yarrowialipolytica growing in olive mill wastewater-based media under aseptic and non-aseptic conditions. Eng. Life Sci. 2017, 17, 695-709. [CrossRef]

4. Sarris, D.; Rapti, A.; Papafotis, N.; Koutinas, A.A.; Papanikolaou, S. Production of Added-Value Chemical Compounds through Bioconversions of Olive-Mill Wastewaters Blended with Crude Glycerol by a Yarrowia lipolytica Strain. Molecules 2019, 24, 222. [CrossRef] [PubMed]

5. Hamdi, M. Toxicity and biodegradability of olive mill wastewaters in batch anaerobic digestion. Appl. Biochem. Biotechnol. 1992, 37, 155-163. [CrossRef]

6. Zervakis, G.; Balis, C. Bioremediation of olive mill wastes water through the production of fungal biomass. In Proceedings of the Second International Conference on Mushrooms Biology and Mushrooms Products, University Park, PA, USA, 9-12 June 1996; pp. 311-323.

7. Tsioulpas, A.; Dimou, D.; Iconomou, D.; Aggelis, G. Phenolic removal in olive oil mill wastewater by strains of Pleurotus spp. in respect to their phenol oxidase (laccase) activity. Bioresour. Technol. 2002, 84, 251-257. [CrossRef]

8. Aggelis, G.; Iconomou, D.; Christou, M.; Bokas, D.; Kotzailias, S.; Christou, G.; Tsagou, V.; Papanikolaou, S. Phenolic removal in a model olive oil mill wastewater using Pleurotus ostreatus in bioreactor cultures and biological evaluation of the process. Water Res. 2003, 37, 3897-3904. [CrossRef]

9. Crognale, S.; Federici, F.; Petruccioli, M. $\beta$-Glucan production by Botryosphaeria rhodina on undiluted olive-mill waste waters. Biotechnol. Lett. 2003, 25, 2013-2015. [CrossRef] [PubMed]

10. D'Annibale, A.; Sermani, G.G.; Federici, F.; Petruccioli, M. Olive-mill wastewaters: A promising substrate for microbial lipase production. Bioresour. Technol. 2006, 97, 1828-1833. [CrossRef] [PubMed]

11. Papanikolaou, S.; Galiotou-Panayotou, M.; Fakas, S.; Komaitis, M.; Aggelis, G. Citric acid production by Yarrowia lipolytica cultivated on olive-mill wastewater-based media. Bioresour. Technol. 2008, 99, 2419-2428. [CrossRef]

12. Sarris, D.; Papanikolaou, S. Biotechnological production of ethanol: biochemistry, processes and technologies. Eng. Life Sci. 2016, 16, 307-329. [CrossRef]

13. Rivaldi, J.D.; Sarrouh, B.F.; da Silva, S.S. Development of biotechnological processes using glycerol from biodiesel production. In Current Research Topics in Applied Microbiology and Microbial Biotechnology; Mendez-Vilas, A., Ed.; World Scientific Publishing Co. Formatex Research Center: Madrid, Spain, 2009; pp. 429-433.

14. Wen, Z.; Pyle, D.J.; Athalye, S.K. Glycerol waste from biodiesel manufacturing. In Microbial Conversions of Raw Glycerol; Aggelis, G., Ed.; Nova Science Publishers Inc.: New York, NY, USA, 2009; pp. 1-7.

15. Monteiro, M.R.; Kugelmeier, C.L.; Pinheiro, R.S.; Batalha, M.O.; da Silva César, A. Glycerol from biodiesel production: Technological paths for sustainability. Ren. Sustain. Energy Rev. 2018, 88, 109-122. [CrossRef]

16. Bellou, S.; Moustogianni, A.; Makri, A.; Aggelis, G. Lipids containing polyunsaturated fatty acids synthesized by Zygomycetes grown on glycerol. Appl. Biochem. Biotechnol. 2012, 166, 146-158. [CrossRef] [PubMed]

17. Dedyukhina, E.G.; Chistyakova, T.I.; Kamzolova, S.V.; Vinter, M.V.; Vainshtein, M.B. Arachidonic acid synthesis by glycerol-grown Mortierella alpina. Eur. J. Lipid Sci. Technol. 2012, 114, 833-841. [CrossRef] 
18. Dedyukhina, E.G.; Chistyakova, T.I.; Mironov, A.A.; Kamzolova, S.V.; Morgunov, I.G.; Vainshtein, M.B. Arachidonic acid synthesis from biodiesel-derived waste by Mortierella alpina. Eur. J. Lipid Sci. Technol. 2014, 116, 429-437. [CrossRef]

19. Fontanille, P.; Kumar, V.; Christophe, G.; Nouaille, R.; Larroche, C. Bioconversion of volatile fatty acids into lipids by the oleaginous yeast Yarrowia lipolytica. Bioresour. Technol. 2012, 114, 443-449. [CrossRef] [PubMed]

20. Xu, J.; Zhao, X.; Wang, W.; Du, W.; Liu, D. Microbial conversion of biodiesel byproduct glycerol to triacylglycerols by oleaginous yeast Rhodosporidium toruloides and the individual effect of some impurities on lipid production. Biochem. Eng. J. 2012, 65, 30-36. [CrossRef]

21. Chang, G.; Luo, Z.; Gu, S.; Wu, Q.; Chang, M.; Wang, X. Fatty acid shifts and metabolic activity changes of Schizochyrium sp. S31 cultured on glycerol. Bioresour. Technol. 2013, 142, 255-260. [CrossRef] [PubMed]

22. Cui, Y.; Blackburn, J.W.; Liang, Y. Fermentation optimization for the production of lipid by Cryptococcus curvatus: Use of response surface methodology. Biomass Bioenergy 2012, 47, 410-417. [CrossRef]

23. Yang, X.; Jin, G.; Gong, Z.; Shen Bai, F.; Zhao, Z.K. Recycling biodiesel-derived glycerol by the oleaginous yeast Rhodosporidium toruloides Y4 through the two-stage lipid production process. Biochem. Eng. J. 2014, 91 , 86-91. [CrossRef]

24. Bommareddy, R.R.; Sabra, W.; Maheshwari, G.; Zeng, A.P. Metabolic network analysis and experimental study of lipid production in Rhodosporidium toruloides grown on single and mixed substrates. Microb. Cell Fact. 2015, 14, 36. [CrossRef] [PubMed]

25. Tchakouteu, S.S.; Kalantzi, O.; Gardeli, C.; Koutinas, A.A.; Aggelis, G.; Papanikolaou, S. Lipid production by yeasts growing on biodiesel-derived crude glycerol: Strain selection and impact of substrate concentration on the fermentation efficiency. J. Appl. Microbiol. 2015, 118, 911-927. [CrossRef] [PubMed]

26. Rymowicz, W.; Rywińska, A.; Źarowska, B.; Juszczyk, P. Citric acid production from raw glycerol by acetate mutants of Yarrowia lipolytica. Chem. Pap. 2006, 60, 391-394. [CrossRef]

27. Rymowicz, W.; Fatykhova, A.R.; Kamzolova, S.V.; Rywinska, A.; Morgunov, I.G. Citric acid production from glycerol-containing waste of biodiesel industry by Yarrowia lipolytica in batch, repeated batch, and cell recycle regimes. Appl. Microbiol. Biotechnol. 2010, 87, 971-979. [CrossRef]

28. Papanikolaou, S.; Beopoulos, A.; Koletti, A.; Thevenieau, F.; Koutinas, A.A.; Nikaud, J.M.; Aggelis, G. Importance of the methyl-citrate cycle on glycerol metabolism in the yeast Yarrowia lipolytica. J. Biotechnol. 2013, 168, 303-314. [CrossRef] [PubMed]

29. Rywińska, A.; Rymowicz, W. High-yield production of citric acid by Yarrowia lipolytica on glycerol in repeated-batch bioreactors. J. Ind. Microbiol. Biotechnol. 2010, 37, 431-435. [CrossRef] [PubMed]

30. Rywińska, A.; Rymowicz, W.; Zarowska, B.; Skrzypiński, A. Comparison of citric acid production from glycerol and glucose by different strains of Yarrowia lipolytica. World J. Microbiol. Biotechnol. 2010, 26, 1217-1224. [CrossRef] [PubMed]

31. Rywińska, A.; Rymowicz, W.; Marcinkiewicz, M. Valorization of raw glycerol for citric acid production by Yarrowia lipolytica yeast. Electron. J. Biotechnol. 2010, 13, 9-10. [CrossRef]

32. Kamzolova, S.V.; Fatykhova, A.R.; Dedyukhina, E.G.; Anastassiadis, S.G.; Golovchenko, N.P.; Morgunov, I.G. Citric acid production by yeast grown on glycerol-containing waste from biodiesel industry. Food Technol. Biotechnol. 2011, 49, 65-74.

33. Tomaszewska, L.; Rywińska, A.; Gładkowski, W. Production of erythritol and mannitol by Yarrowia lipolytica yeast in media containing glycerol. J. Ind. Microbiol. Biotechnol. 2012, 39, 1333-1343. [CrossRef]

34. Rywińska, A.; Tomaszewska, L.; Rymowicz, W. Erythritol biosynthesis by Yarrowia lipolytica yeast under various culture conditions. Afr. J. Microbiol. Res. 2013, 7, 3511-3516.

35. Papanikolaou, S.; Rontou, M.; Belka, A.; Athenaki, M.; Gardeli, C.; Mallouchos, A.; Kalantzi, O.; Koutinas, A.A.; Kookos, I.K.; Zeng, A.P.; et al. Conversion of biodiesel-derived glycerol into biotechnological products of industrial significance by yeast and fungal strains. Eng. Life Sci. 2017, 17, 262-281. [CrossRef]

36. Papanikolaou, S.; Aggelis, G. Biotechnological valorization of biodiesel derived glycerol waste through production of single cell oil and citric acid by Yarrowia lipolytica. Lipid Technol. 2009, 21, 83-87. [CrossRef]

37. Abghari, A.; Chen, S. Yarrowia lipolytica as an oleaginous cell factory platform for production of fatty acid-based biofuel and bioproducts. Front. Energy Res. 2014, 2, 21. [CrossRef]

38. Papanikolaou, S.; Chevalot, I.; Komaitis, M.; Marc, I.; Aggelis, G. Single cell oil production by Yarrowia lipolytica growing on an industrial derivative of animal fat in batch cultures. Appl. Microbiol. Biotechnol. 2002, 58, 308-312. [CrossRef] [PubMed] 
39. Rymowicz, W.; Rywinska, A.; Gladowski, W. Simultaneous production of citric acid and erythritol from crude glycerol by Yarrowia lipolytica Wratislavia K1. Chem. Pap. 2008, 62, 239-246. [CrossRef]

40. Rymowicz, W.; Rywinska, A.; Marcinkiewicz, M. High yield production of erythritol from raw glycerol in fed-batch cultures of Yarrowia lipolytica. Biotechnol. Lett. 2009, 31, 377-380. [CrossRef] [PubMed]

41. Rywińska, A.; Juszczyk, P.; Wojtatowicz, M.; Rymowicz, W. Chemostat study of citric acid production from glycerol by Yarrowia lipolytica. J. Biotechnol. 2011, 152, 54-57. [CrossRef] [PubMed]

42. Chatzifragkou, A.; Makri, A.; Belka, A.; Bellou, S.; Mavrou, M.; Mastoridou, M.; Mystrioti, P.; Onjaro, G.; Aggelis, G.; Papanikolaou, S. Biotechnological conversions of biodiesel derived waste glycerol by yeast and fungal species. Energy 2011, 36, 1097-1108. [CrossRef]

43. Chatzifragkou, A.; Petrou, I.; Gardeli, C.; Komaitis, M.; Papanikolaou, S. Effect of Origanum vulgare L. essential oil on growth and lipid profile of Yarrowia lipolytica cultivated on glycerol-based media. J. Am. Oil Chem. Soc. 2011, 88, 1955-1964. [CrossRef]

44. Morgunov, I.G.; Kamzolova, S.V.; Lunina, J.N. The citric acid production from raw glycerol by Yarrowia lipolytica yeast and its regulation. Appl. Microbiol. Biotechnol. 2013, 97, 7387-7397. [CrossRef] [PubMed]

45. Huang, C.; Chen, X.; Xiong, L.; Chen, X.; Ma, L.; Chen, Y. Single cell oil production from low-cost substrates: The possibility and potential of its industrialization. Biotechnol. Adv. 2013, 31, 129-139. [CrossRef] [PubMed]

46. Aly, A.A.; Hasan, Y.N.; Al-Farraj, A.S. Olive mill wastewater treatment using a simple zeolite-based low-cost method. J. Environ. Manag. 2014, 145, 341-348. [CrossRef] [PubMed]

47. Papanikolaou, S.; Chevalot, I.; Komaitis, M.; Aggelis, G.; Marc, I. Kinetic profile of the cellular lipid composition in an oleaginous Yarrowia lipolytica apable of producing a cocoa-butter substitute from industrial fats. Antonie Leeuwenhoek 2001, 80, 215-224. [CrossRef] [PubMed]

48. Papanikolaou, S.; Muniglia, L.; Chevalot IAggelis, G.; Marc, I. Accumulation of cocoa-butter-like lipid by Yarrowia lipolytica cultivated on agro-industrial residues. Curr. Microbiol. 2003, 46, 124-130. [CrossRef] [PubMed]

49. Xiong, D.; Zhang, H.; Xie, Y.; Tang, N.; Berenjian, A.; Song, Y. Conversion of mutton fat to cocoa butter equivalent by increasing the unsaturated fatty acids at the sn-2 position of triacylglycerol through fermentation by Yarrowia lipolytica. Am. J. Biochem. Biotechnol. 2015, 11, 57-65. [CrossRef]

50. Zhao, L.; Li, B.; Xiong, D.; Zhang, H.; Song, Y.; Yang, S. Cocoa-butter equivalent production from Yarrowia lipolytica by optimization of fermentation technology. Am. J. Biochem. Biotechnol. 2016, 12, 196-205. [CrossRef]

51. Papanikolaou, S.; Aggelis, G. Yarrowia lipolytica: A model microorganism used for the production of tailor-made lipids. Eur. J. Lipid Sci. Technol. 2010, 112, 639-654. [CrossRef]

52. Poli, J.S.; da Silva, M.A.N.; Siqueira, E.P.; Pasa, V.M.D.; Rosa, C.A.; Valente, P. Microbial lipid produced by Yarrowia lipolytica QU21 using industrial waste: A potential feedstock for biodiesel production. Bioresour. Technol. 2014, 161, 320-326. [CrossRef]

53. Papanikolaou, S.; Chatzifragkou, A.; Fakas, S.; Galiotou-Panayotou, M.; Komaitis, M.; Nicaud, J.M.; Aggelis, G. Biosynthesis of lipids and organic acids by Yarrowia lipolytica strains cultivated on glucose. Eur. J. Lipid Sci. Technol. 2009, 111, 1221-1232. [CrossRef]

54. Juszczyk, P.; Rymowicz, W. Characterization of microbial biomass production from glycerin waste by various yeast strains. In Microbial Conversions of Raw Glycerol; Aggelis, G., Ed.; Nova Science Publishers: New York, NY, USA, 2009; pp. 125-135.

55. Celińska, E.; Grajek, W. A novel multigene expression construct for modification of glycerol metabolism in Yarrowia lipolytica. Microb. Cell Fact. 2013, 12, 102. [CrossRef] [PubMed]

56. Rakicka, M.; Kieron, A.; Hapeta, P.; Neuvéglise, C.; Lazar, Z. Sweet and sour potential of yeast from the Yarrowia clade. Biomass Bioenergy 2016, 92, 48-54. [CrossRef]

57. Rakicka, M.; Rywinska, A.; Cybulski, K.; Rymowicz, W. Enhanced production of erythritol and mannitol by Yarrowia lipolytica in media containing surfactants. Braz. J. Microbiol. 2016, 47, 417-423. [CrossRef] [PubMed]

58. Dourou, M.; Kancelista, A.; Juszczyk, P.; Sarris, D.; Bellou, S.; Triantaphyllidou, I.E.; Rywinska, A.; Papanikolaou, S.; Aggelis, G. Bioconversion of olive mill wastewater into high-added value products. J. Clean. Prod. 2016, 139, 957-969. [CrossRef]

59. Tomaszewska, L.; Rakicka, M.; Rymowicz, W.; Rywińska, A. A comparative study on glycerol metabolism to erythritol and citric acid in Yarrowia lipolytica yeast cells. FEMS Yeast Res. 2014, 14, 966-976. [CrossRef] 
60. Rzechonek, D.A.; Dobrowolski, A.; Rymowicz, W.; Mirończuk, A.M. Aseptic production of citric and isocitric acid from crude glycerol by genetically modified Yarrowia lipolytica. Bioresour. Technol. 2019, 271, 340-344. [CrossRef] [PubMed]

61. Papanikolaou, S.; Fakas, S.; Fick, M.; Chevalot, I.; Galiotou-Panayotou, M.; Komaitis, M.; Marc, I.; Aggelis, G. Biotechnological valorisation of raw glycerol discharged after bio-diesel (fatty acid methyl esters) manufacturing process: Production of 1, 3-propanediol, citric acid and single cell oil. Biomass Bioenergy 2008, 32, 60-71. [CrossRef]

62. Sarris, D.; Galiotou-Panayotou, M.; Koutinas, A.A.; Komaitis, M.; Papanikolaou, S. Citric acid, biomass and cellular lipid production by Yarrowia lipolytica strains cultivated on olive mill wastewater-based media. J. Chem. Technol. Biotechnol. 2011, 86, 1439-1448. [CrossRef]

63. Papanikolaou, S.; Kampisopoulou, E.; Blanchard, F.; Rondags, E.; Gardeli, C.; Koutinas, A.A.; Aggelis, G. Production of secondary metabolites through glycerol fermentation under carbon-excess conditions by the yeasts Yarrowia lipolytica and Rhodosporidium toruloides. Eur. J. Lipid Sci. Technol. 2017, 119, 1600507. [CrossRef]

64. Papanikolaou, S.; Aggelis, G. Lipids of oleaginous yeasts. Part II: Technology and potential applications. Eur. J. Lipid Sci. Technol. 2011, 113, 1052-1073. [CrossRef]

65. Dobrowolski, A.; Mituła, P.; Rymowicz, W.; Mirończuk, A.M. Efficient conversion of crude glycerol from various industrial wastes into single cell oil by yeast Yarrowia lipolytica. Bioresour. Technol. 2016, 207, 237-243. [CrossRef] [PubMed]

66. Papanikolaou, S.; Aggelis, G. Sources of microbial oils with emphasis to Mortierella (Umbelopsis) isabellina fungus. World J. Microbiol. Biotechnol. 2019, 35, 63. [CrossRef] [PubMed]

67. Makri, A.; Fakas, S.; Aggelis, G. Metabolic activities of biotechnological interest in Yarrowia lipolytica grown on glycerol in repeated batch cultures. Bioresour. Technol. 2010, 101, 2351-2358. [CrossRef] [PubMed]

68. Ratledge, C. Yeasts, moulds, algae and bacteria as sources of lipids. In Technological Advances in Improved and Alternative Sources of Lipids; Kamel, B.S., Kakuda, Y., Eds.; Blackie Academic and Professional: London, UK, 1994; pp. 235-291.

69. Paramithiotis, S.; Muller, M.R.A.; Ehrmann, M.A.; Tsakalidou, E.; Seiler, H.; Vogel, R.; Kalantzopoulos, G. Polyphasic identification of wild yeast strains isolated from Greek sourdoughs. Syst. Appl. Microbiol. 2000, 23, 156-164. [CrossRef]

70. Liang, Y.N.; Sarkany, N.; Cui, Y.; Blackburn, J.W. Batch stage study of lipid production from crude glycerol derived from yellow grease or animal fats through microagal fermentation. Bioresour. Technol. 2010, 101, 6745-6750. [CrossRef]

71. Miller, G. Determination of reducing sugar by DNS method. Anal. Chem. 1959, 31, 426-428. [CrossRef]

72. Kachrimanidou, V.; Kopsahelis, N.; Chatzifragkou, A.; Papanikolaou, S.; Yanniotis, S.; Kookos, I.K.; Koutinas, A.A. Utilisation of by-products from sunflower-based biodiesel production processes for the production of fermentation feedstock. Waste Biomass Valor. 2013, 4, 529-537. [CrossRef]

73. Zikou, E.; Chatzifragkou, A.; Koutinas, A.A.; Papanikolaou, S. Evaluating glucose and xylose as cosubstrates for lipid accumulation and $\gamma$-linolenic acid biosynthesis of Thamnidium elegans. J. Appl. Microbiol. 2013, 114, 1020-1032. [CrossRef]

74. Sayadi, S.; Ellouz, R. Decolourization of olive mill waste-waters by the white-rot fungus Phanerochaete chrysosporium: Involvement of the lignin-degrading system. Appl. Microbiol. Biotechnol. 1992, 37, 813-817. [CrossRef]

(C) 2019 by the authors. Licensee MDPI, Basel, Switzerland. This article is an open access article distributed under the terms and conditions of the Creative Commons Attribution (CC BY) license (http://creativecommons.org/licenses/by/4.0/). 

Article

\title{
Long-Term Evaluation of Mesophilic Semi-Continuous Anaerobic Digestion of Olive Mill Solid Waste Pretreated with Steam-Explosion
}

\author{
Antonio Serrano ${ }^{1,2}$, Fernando G. Fermoso 1,*, Bernabé Alonso-Fariñas ${ }^{3}$, \\ Guillermo Rodríguez-Gutiérrez ${ }^{1}$, Sergio López ${ }^{1,4}$, Juan Fernandez-Bolaños ${ }^{1}$ and Rafael Borja ${ }^{1}$ \\ 1 Instituto de la Grasa (CSIC), Campus Universitario Pablo de Olavide, Edificio 46, Ctra. de Utrera km 1, \\ 41013 Sevilla, Spain; a.serranomoral@uq.edu.au (A.S.); guirogu@ig.csic.es (G.R.-G.); serglom@us.es (S.L.); \\ jfbg@cica.es (J.F.-B.); rborja@cica.es (R.B.) \\ 2 School of Civil Engineering, The University of Queensland, Campus St. Lucia-AEB Ed 49, St Lucia, \\ QLD 4067, Australia \\ 3 Department of Chemical and Environmental Engineering, University of Sevilla, Higher Technical School of \\ Engineering, Camino de los Descubrimientos, s/n, 41004 Sevilla, Spain; bernabeaf@us.es \\ 4 Department of Cell Biology, University of Sevilla, 41004 Sevilla, Spain \\ * Correspondence: fgfermoso@ig.csic.es
}

Received: 23 May 2019; Accepted: 10 June 2019; Published: 11 June 2019

\begin{abstract}
Steam-explosion is a promising technology for recovering phenolic compounds from olive mill solid waste (OMSW) due to its high impact on the structure of the fibre. Moreover, the recovery of the phenols, which are well-known microbial inhibitors, could improve the subsequent biomethanization of the dephenolized OMSW to produce energy. However, there is a considerable lack of knowledge about how the remaining phenolic compounds could affect a long-term biomethanization process of steam-exploded OMSW. This work evaluated a semi-continuous mesophilic anaerobic digestion of dephenolized steam-exploited OMSW during a long operational period (275 days), assessing different organic loading rates (OLRs). The process was stable at an OLR of $1 \mathrm{gVS} /(\mathrm{L} \cdot \mathrm{d})$, with a specific production rate of $163 \pm 28 \mathrm{~mL} \mathrm{CH}_{4} /(\mathrm{gVS} \cdot \mathrm{d})$. However, the increment of the OLR up to $2 \mathrm{gVS} /(\mathrm{L} \cdot \mathrm{d})$ resulted in total exhaust of the methane production. The increment in the propionic acid concentration up to $1486 \mathrm{mg} / \mathrm{L}$ could be the main responsible factor for the inhibition. Regardless of the OLR, the concentration of phenolic compounds was always lower than the inhibition limits. Therefore, steam-exploited OMSW could be a suitable substrate for anaerobic digestion at a suitable OLR.
\end{abstract}

Keywords: anaerobic digestion; economic evaluation; olive mill solid waste; phenolic compounds; steam explosion

\section{Introduction}

The olive oil sector stands as an important economic sector for many countries; however, some current challenges could risk its economical sustainability. On one hand, the olive oil industry has to compete with other low-cost vegetable oils, such as sunflower or palm oil. On the other hand, it is required to develop sustainable and environmentally friendly management for the olive mill solid waste (OMSW) generated during extraction [1]. The OMSW results from the two-phase olive oil manufacturing process, being a high-humidity and polluting waste, where the remains of olive fruit are retained. Each ton of olive oil generates around 4 tons of OMSW each year, and due its high potential impact on the environment [2,3], it is necessary to develop an adequate OMSW management strategy to ensure environmental protection, but also economic sustainability of the olive oil sector.

One of the main processes for valorisation of OMSW is the recovery of bioactive compounds contained in its structure, which can be used in food industry, pharmacy or cosmetics. In fact, 
most of the bioactive compounds present in the olives remains in the OMSW [4,5]. These bioactive compounds, which are the responsible for the healthy properties of olive oil, are mainly phenols such as hydroxytyrosol, tyrosol, vanillic acid, p-coumaric acid, 3,4-dihydroxyphenylglycol, etc. [4,6]. Most of these compounds form part of complex structures, such as the cell wall, and it is therefore necessary to carry out a previous solubilisation step to recover them [7]. Thermal treatments have been shown to facilitate the solubilisation of the valuable bioactive compounds to a liquid phase, where they can be easily recovered [6].

Among the different thermal treatments, steam-explosion allows higher breakdown of the lignocellulosic structures compared to other conventional and less aggressive thermal treatments [8]. During the steam-explosion process, the material is heated with high pressure saturated steam for a short period of time followed by a fast pressure reduction, which causes an explosive decompression. During steam-explosion of OMSW, hemicellulose and cellulose structures are mainly hydrolysed, whereas the lignin is also affected to a certain degree [4]. The breakdown of the lignocellulosic structures through the steam-explosion treatment results in a high solubilisation of bioactive compounds that can be easily recovered [9].

The steam-exploded OMSW that remains after bioactive compound extraction still needs to be stabilized to avoid environmental pollution. Among the processes to stabilize OMSW, anaerobic digestion offers the possibility of obtaining methane, as an energy carrier, and the possibility to recover the remaining digestate as fertilizer [10,11]. According to Stoyanova et al. [12], stable single-stage semi-continuous OMSW anaerobic digestion proceeded at an organic loading rate (OLR) of $0.76 \mathrm{kgVS} /\left(\mathrm{m}^{3} \mathrm{~d}\right)(\mathrm{VS}$, total volatile solids). Recently, Serrano et al. [13] determined that the accumulation of VFA was the main reason for destabilization during anaerobic digestion of OMSW subjected to a thermal pre-treatment $\left(1 \mathrm{~h}\right.$ at $\left.170{ }^{\circ} \mathrm{C}\right)$ at an OLR of $2 \mathrm{gVS} /\left(\mathrm{m}^{3} \cdot \mathrm{d}\right)$. Inhibition of the anaerobic digestion of lignocellulosic material has been also associated with phenols and derived phenolic compounds because of their severe antimicrobial properties [14,15]. Some phenols and furans with high inhibitory properties, such as hydroxymethylfurfural or furfural, are generated during the thermal treatment of the lignocellulosic compounds in severe conditions $[8,16]$.

Extraction of polyphenols can enable the utilization of OMSW and its valorisation with energy recovery by anaerobic digestion due to the partial removal of inhibitors [3,9]. A recent previous study showed that a steam-explosion pre-treatment of OMSW $\left(200{ }^{\circ} \mathrm{C}\right.$ for $5 \mathrm{~min}$, prior to a rapid decompression) allowed the recovery of more than $2000 \mathrm{mg}$ hydroxytyrosol $/ \mathrm{kg}$ of steam-exploded OMSW [9]. In the subsequent anaerobic digestion step of the steam-exploded OMSW performed in the batch mode, pre-treatment and phenol extraction improved the methane yield coefficient and methane production rate of the dephenolized liquid fraction by $26.8 \%$ and $26.4 \%$, respectively, with respect to untreated OMSW [9]. However, more information about the performance of long-term anaerobic digestion of steam-exploded OMSW in semi-continuous mode, and how the accumulation of potential toxic compounds could affect the process stability and biogas production, is still required prior to industrial implementation.

Therefore, due to the potential of the mentioned OMSW steam-explosion pre-treatment [9], the present work aimed to assess the semi-continuous mesophilic anaerobic digestion of steam-exploded OMSW during a long operational period (275 days) assessing different OLRs. Finding the adequate OLR is very important for optimizing process efficiency and, at the same time, reducing the risk of destabilization.

\section{Materials and Methods}

\subsection{Substrate and Inoculum}

The substrate used in the present study consisted of OMSW, which was subjected to a phenol recovery process. Raw OMSW was obtained from the OMSW management plant "Oleícola El Tejar" located in Marchena (Seville), Spain. The phenol recovery process entailed a steam-explosion 
pre-treatment $\left(200^{\circ} \mathrm{C}\right.$ for $5 \mathrm{~min}$, prior to a rapid decompression). Subsequent phenol recovery was done by a patented chromatographic system (WO 2013/007850A1) from the generated liquid phase (detailed in Serrano et al. [9]). The treated OMSW used in the present research was composed of the pre-treated solid phase and the dephenolized liquid phase. Analytical characterization of the used OMSW is shown in Table 1.

Table 1. Characterization of the Olive Mill Solid Waste (OMSW).

\begin{tabular}{ccc}
\hline TS & $\mathrm{g} / \mathrm{L}$ & $72.7 \pm 0.8$ \\
VS & $\mathrm{g} / \mathrm{L}$ & $68.4 \pm 0.8$ \\
COD & $\mathrm{g} / \mathrm{L}$ & $80.6 \pm 2.6$ \\
sCOD & $\mathrm{g} / \mathrm{L}$ & $47.5 \pm 0.3$ \\
$\mathrm{pH}$ & & $4.49 \pm 0.05$ \\
Alkalinity & $\mathrm{mg} \mathrm{CaCo} / \mathrm{L}$ & - \\
Acetic acid & $\mathrm{mg} / \mathrm{L}$ & $1802 \pm 8$ \\
Total Phenols & $\mathrm{mg} \mathrm{Gallic} \mathrm{Acid/L}$ & $4400 \pm 149$ \\
Soluble Phenols & $\mathrm{mg} \mathrm{Gallic} \mathrm{Acid/L}$ & $1373 \pm 66$ \\
3,4-Dihydroxyphenylglycol & $\mathrm{mg} / \mathrm{L}$ & $22.0 \pm 2.5$ \\
Hydroxymethylfurfural & $\mathrm{mg} / \mathrm{L}$ & $85.1 \pm 6.1$ \\
Hydroxytyrosol & $\mathrm{mg} / \mathrm{L}$ & $513.8 \pm 25.4$ \\
Tyrosol & $\mathrm{mg} / \mathrm{L}$ & $92.9 \pm 14.1$ \\
Homovanillic acid & $\mathrm{mg} / \mathrm{L}$ & $1.16 \pm 0.09$ \\
Vanillic acid & $\mathrm{mg} / \mathrm{L}$ & $7.7 \pm 0.9$ \\
Vanillin & $\mathrm{mg} / \mathrm{L}$ & $188.7 \pm 27.8$ \\
4-Ethylphenol & $\mathrm{mg} / \mathrm{L}$ & $\mathrm{n} . \mathrm{d}$. \\
\hline
\end{tabular}

A full-scale digester treating sewage sludge at the "COPERO" wastewater treatment plant (Seville, Spain) was the source of the used anaerobic inoculum (Total Solids (TS) $=21 \pm 1 \mathrm{~g} / \mathrm{kg}$ and $\mathrm{VS}=19 \pm 1 \mathrm{~g} / \mathrm{kg}$ ). The reactors were initially inoculated at a concentration of $10 \mathrm{gVS} / \mathrm{L}$.

\subsection{Anaerobic Digestion Experimental Procedure}

Two-litre continuously stirred tank reactors (CSTRs) in triplicate were used to evaluate mesophilic semi-continuous anaerobic digestion of the steam-exploded OMSW (more details in [13]). The volume of methane was measured daily after the removal of the $\mathrm{CO}_{2}$ with tightly closed bubblers containing a $\mathrm{NaOH}$ solution $(3 \mathrm{~N})$. The remaining gas volume was measured by liquid displacement using Boyle-Mariotte flasks. The volume of methane was expressed at standard temperature and pressure conditions $\left(0^{\circ} \mathrm{C}\right.$ and $\left.1 \mathrm{~atm}\right)$.

Table 2 summarizes the experimental design, including the stages for bio-stimulation and the adaptation to the substrate. After the stimulation and adaptation periods, anaerobic digestion performance of the steam-exploded OMSW was evaluated during a long-term period of 275 days at different organic loading rates (OLR). Samples were analyzed at least twice per week.

Table 2. Experimental design.

\begin{tabular}{cccc}
\hline Experimental Stage & Duration & Feeded Substrate & OLR (gVS/(L·d)) \\
\hline Bio-stimulation & 15 days & SS & $0.5-1$ \\
\hline \multirow{2}{*}{ Adaptation } & 15 days & SS:OMSW 75:25, in VS & 2 \\
& 15 days & SS:OMSW 50:50, in VS & 2 \\
Stage 1 & 15 days & SS:OMSW 25:75, in VS & 2 \\
Stage 2 & 75 days (days 0-75) & OMSW & 2 \\
Stage 3 & 100 days (days 75-175) & OMSW & 1 \\
\hline
\end{tabular}

SS, Synthetic solution with glucose $(50 \mathrm{~g} / \mathrm{L})$ and sodium acetate $(25.2 \mathrm{~g} / \mathrm{L})$. 


\subsection{Chemical Analyses}

TS, VS, pH and alkalinity were analysed following the recommendations of the American Public Health Association (APHA) [17]. Total chemical oxygen demand (COD; g/ $/ \mathrm{kg}$ ) was measured as described by Raposo et al. (2008), while the colorimetric standard method 5220D was used for the measurement of the soluble COD (sCOD; $\mathrm{g} / \mathrm{L}$ or $\mathrm{mg} / \mathrm{L}$ ) [17]. Total phenols were quantified by spectrophotometry at $655 \mathrm{~nm}$ through the Folin-Ciocalteu method [5]. A gallic acid curve was used for calibration of the method. Soluble phenols were also quantified by the Folin-Ciocalteu method after filtration through a Millipore $0.45 \mu \mathrm{m}$ filter (Albet GF $47 \mathrm{~mm}$, Dassel, Germany). Individual phenols were quantified using a Hewlett-Packard 1100 liquid chromatography system (more details are described in Serrano et al. [9]). Separate volatile fatty acids $\left(\mathrm{C}_{2}-\mathrm{C}_{6}\right)$ were determined using a Shimadzu gas chromatograph (GC-2010).

\subsection{Microbial Analysis}

Samples were taken at the inoculum and at 75, 175, and 250 days of the experiment in order to analyze the number of microorganisms by DAPI staining. Diluted samples of $10 \mathrm{~L}$ volume were fixed onto glass slides for $20 \mathrm{~min}$, and then mounted with $25 \mathrm{~L}$ of Fluoroshield with DAPI (Abcam, Cambridge, UK) prior to visualization in a Zeiss Axio Imager M2 fluorescence microscope under UV light. Images were acquired with an AxioCam MRm digital camera. The mean number of cells was determined from 10 random images using the ImageJ software. Backgrounds were subtracted from all images to improve image clarity as described elsewhere [18].

\subsection{Economic Assessment}

The economic profitability of a bio-refinery consisting of steam explosion pre-treatment, phenol extraction, anaerobic digestion and a cogeneration system was assessed (Figure 1). Net present value (NPV), internal rate of return (IRR) and payback were calculated according to the methodology described in Serrano et al. (2017b). An additional natural gas input was considered to cover the thermal energy requirement due to the steam explosion pre-treatment. Although a price of $520 € / \mathrm{kg}$ for phenol extract [19] was initially fixed in the study, a possible drop in this price due to the implementation of the new technology could be considered in the profitability assessment. In this sense, a sensibility study was performed to calculate, making NPV equal to zero, the minimum price for which the bio-refinery project would be profitable. A full and detailed description of the economic assessment methodology is reported in Serrano et al. [3]. Due to the high initial inversion, only the financed project scenario was considered. The discount rate and prices of both sold electricity and bought natural gas were updated to $1.40 \%, 0.12 € / \mathrm{kWh}[20]$, and $0.04 € / \mathrm{kWh}[21]$, respectively.

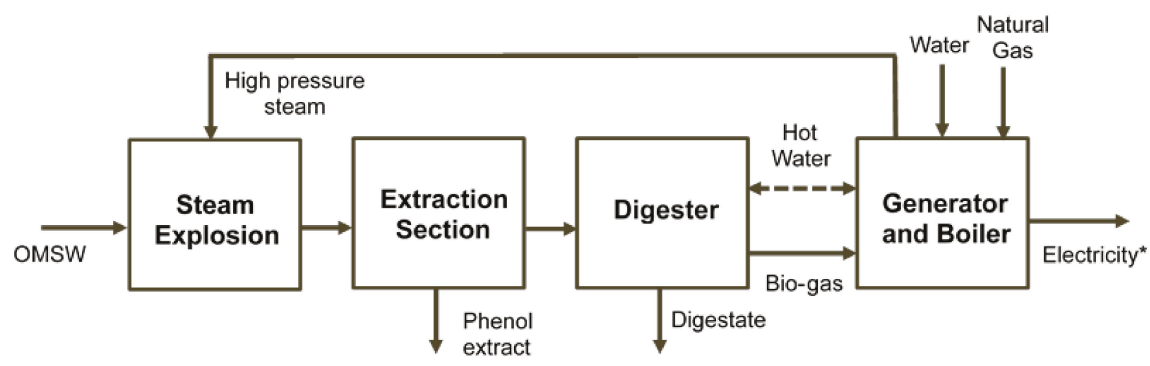

Figure 1. Bio-refinery scheme consisting of steam explosion solubilization, phenol recovery, anaerobic digestion and a cogeneration system. * Net electricity production after subtracting the total electricity consumed from the electricity produced. 


\section{Results and Discussion}

\subsection{Methane Production at the Different Experimental Phases}

The methane production was monitored daily throughout the digestion time for the different OLRs (Figure 2). As can be observed, methane yield varied significantly when varying the OLR. In the first stage, i.e., $0-75$ days and OLR $=2 \mathrm{gVS} /(\mathrm{L} \cdot \mathrm{d})$, the methane production rate presented a mean value of around $0.369 \pm 0.049 \mathrm{gCOD} \mathrm{CH}_{4} /(\mathrm{gCOD} \cdot \mathrm{d})$, i.e., $152 \pm 21 \mathrm{~mL} \mathrm{CH}_{4} /(\mathrm{gVS} \cdot \mathrm{d})$ (Table 3).

Table 3. Methane production rates, $\mathrm{pH}$, alkalinity, COD, CODs, total phenols, soluble phenols, total VFA and individual phenolic compound concentration during the different organic loading rates (OLRs).

\begin{tabular}{|c|c|c|c|c|}
\hline & & Stage 1 & Stage 2 & Stage 3 \\
\hline OLR & $\operatorname{gVS} /\left(L^{*} \mathrm{~d}\right)$ & 2 & 1 & 2 \\
\hline Days & & $0-75$ & 75-175 & $175-275$ \\
\hline Methane production rate & $\mathrm{mL} \mathrm{CH}_{4} /\left(\mathrm{gVS}^{*} \mathrm{~d}\right)$ & $152 \pm 21$ & $163 \pm 28$ & $85 \pm 59($ from 175 to 8$)$ \\
\hline Methane production rate & $\mathrm{gCOD}^{-\mathrm{CH}_{4}} /\left(\mathrm{gCOD}^{*} \mathrm{~d}\right)$ & $0.369 \pm 0.049$ & $0.395 \pm 0.066$ & $\begin{array}{c}0.167 \pm 0.126 \\
(\text { from } 0.345 \text { to } 0.030)\end{array}$ \\
\hline $\mathrm{pH}$ & & $7.81 \pm 0.34$ & $7.39 \pm 0.41$ & $6.73 \pm 0.58$ \\
\hline Alkalinity & $\mathrm{mg} \mathrm{CaCO} \mathrm{Ca}_{3} / \mathrm{L}$ & $3033 \pm 894$ & $1869 \pm 694$ & $2704 \pm 902$ \\
\hline CODt & $\mathrm{mgO}_{2} / \mathrm{L}$ & $19290 \pm 5780$ & $21220 \pm 3760$ & $24890 \pm 5500$ \\
\hline sCOD & $\mathrm{mgO}_{2} / \mathrm{L}$ & $4045 \pm 1175$ & $4010 \pm 830$ & $\begin{array}{c}8613 \pm 2733 \\
\text { (from } 4350 \text { to } 11765 \text { ) }\end{array}$ \\
\hline Total phenols & mg Gallic Acid/L & $686 \pm 199$ & $697 \pm 112$ & $1406 \pm 413$ \\
\hline Soluble phenols & mg Gallic Acid/L & $612 \pm 208$ & $676 \pm 93$ & $996 \pm 197$ \\
\hline VFA & $\mathrm{mgO}_{2} / \mathrm{L}$ & $1682 \pm 718$ & $683 \pm 586$ & $4180 \pm 1907$ \\
\hline $\mathrm{C} 2$ & $\mathrm{mgO}_{2} / \mathrm{L}$ & $230 \pm 135$ & $80 \pm 67$ & $1334 \pm 629$ \\
\hline C3 & $\mathrm{mgO}_{2} / \mathrm{L}$ & $1010 \pm 450$ & $251 \pm 318$ & $1486 \pm 670$ \\
\hline iC4 & $\mathrm{mgO}_{2} / \mathrm{L}$ & $71 \pm 64$ & $16 \pm 17$ & $94 \pm 70$ \\
\hline $\mathrm{nC4}$ & $\mathrm{mgO}_{2} / \mathrm{L}$ & $24 \pm 24$ & $32 \pm 64$ & $294 \pm 250$ \\
\hline iC5 & $\mathrm{mgO}_{2} / \mathrm{L}$ & $172 \pm 76$ & $180 \pm 117$ & $406 \pm 97$ \\
\hline $\mathrm{nC5}$ & $\mathrm{mgO}_{2} / \mathrm{L}$ & $119 \pm 64$ & $99 \pm 95$ & $376 \pm 242$ \\
\hline iC6 & $\mathrm{mgO}_{2} / \mathrm{L}$ & < D.L. & $5 \pm 6$ & $<$ D.L. \\
\hline $\mathrm{nC6}$ & $\mathrm{mgO}_{2} / \mathrm{L}$ & $57 \pm 22$ & $18 \pm 24$ & $92 \pm 71$ \\
\hline
\end{tabular}

At day 75, the OLR was set to $1 \mathrm{gVS} /(\mathrm{L} \cdot \mathrm{d})$. From days $75-175$, the mean methane production rate was $0.395 \pm 0.066 \mathrm{gCOD}_{\mathrm{CH} 4} /(\mathrm{gCOD} \cdot \mathrm{d})$, i.e., $163 \pm 28 \mathrm{~mL} \mathrm{CH}_{4} /(\mathrm{gVS} \cdot \mathrm{d})$ (Table 3, Figure 2). This value was just $7.2 \%$ higher than that obtained at the previous $2 \mathrm{gVS} /(\mathrm{L} \cdot \mathrm{d})$ OLR. As the reduction of the OLR did not result in a marked increase in methane production, it could be remarked that the steam-explosion pre-treatment allowed operation at both OLRs without affecting the methane production during the assayed period. Serrano et al. [3] reported that the batch anaerobic digestion of steam-exploded OMSW produced a maximum value of $108 \pm 9 \mathrm{~mL} \mathrm{CH} /(\mathrm{gVS} \cdot \mathrm{d})$, i.e., $40 \%$ and $50 \%$ lower than that obtained in the present research operating at an OLR of 2 and $1 \mathrm{gVS} /(\mathrm{L} \cdot \mathrm{d})$, respectively. The higher total duration of the experiments at the semi-continuous mode, compared with the 25 day operating period of the batch experiments, allowed adaptation of the inoculum to the substrate and a higher methane production was acheived [3].

After 175 days, the OLR feed to the reactors was again augmented to $2 \mathrm{gVS} /(\mathrm{L} \cdot \mathrm{d})$ to evaluate if a long operation time could result in better adaptation of the digesting microorganisms to the substrate or if the accumulation of organic compounds could affect the stability. However, the increment in the OLR entailed a complete failure of the process and a marked decrease of the methane production (Figure 2). Concretely, the values of methane production rate decreased from 0.345 to $0.030 \mathrm{gCOD} \mathrm{CH}_{4} /(\mathrm{gCOD} \cdot \mathrm{d})$, i.e., from 175 to $8 \mathrm{~mL} \mathrm{CH}_{4} /(\mathrm{gVS} \cdot \mathrm{d}$ ) (Table 3). The values of methane production rates in the third stage implied that the OMSW was not efficiently converted in methane. According to the obtained results, the OLR of $1 \mathrm{gVS} /(\mathrm{L} \cdot \mathrm{d})$ was most recommendable for the biomethanization of steam-explosion pre-treated and dephenolized OMSW, due to the more stable operation and higher methane yield and methane 
production rate. Other authors have shown similar effects on the methane production by increasing the OLR. For example, Serrano et al. [13] reported that the methane production in the anaerobic digestion of OMSW subjected to a thermal treatment at $170{ }^{\circ} \mathrm{C}$ and $1 \mathrm{~h}$ varied from $119 \pm 30 \mathrm{~mL} \mathrm{CH} 4 /(\mathrm{gVS} \cdot \mathrm{d})$ to $172 \pm 60 \mathrm{~mL} \mathrm{CH} 4 /(\mathrm{gVS} \cdot \mathrm{d})$ by reducing the OLR from 2 to $1 \mathrm{gVS} /(\mathrm{L} \cdot \mathrm{d})$. Stoyanova et al. [12] reported that the biomethanization of two-phase OMSW operated at stable conditions at an OLR of $0.76 \mathrm{gVS} /(\mathrm{L} \cdot \mathrm{d})$, but the increase of the OLR up to 1.1 and $1.76 \mathrm{gVS} /(\mathrm{L} \cdot \mathrm{d})$ affected the methane production and, finally, caused the total failure of the system. In the same way, Orive et al. [22] also reported low methane production rates $\left(77 \mathrm{~mL} \mathrm{CH}_{4} /(\mathrm{gVS} \cdot \mathrm{d})\right.$ in the semi-continuous anaerobic digestion of diluted OMSW (COD: $60 \mathrm{~g} / \mathrm{kg}$ ) at high OLRs (around $3 \mathrm{gVS} /(\mathrm{L} \cdot \mathrm{d})$.

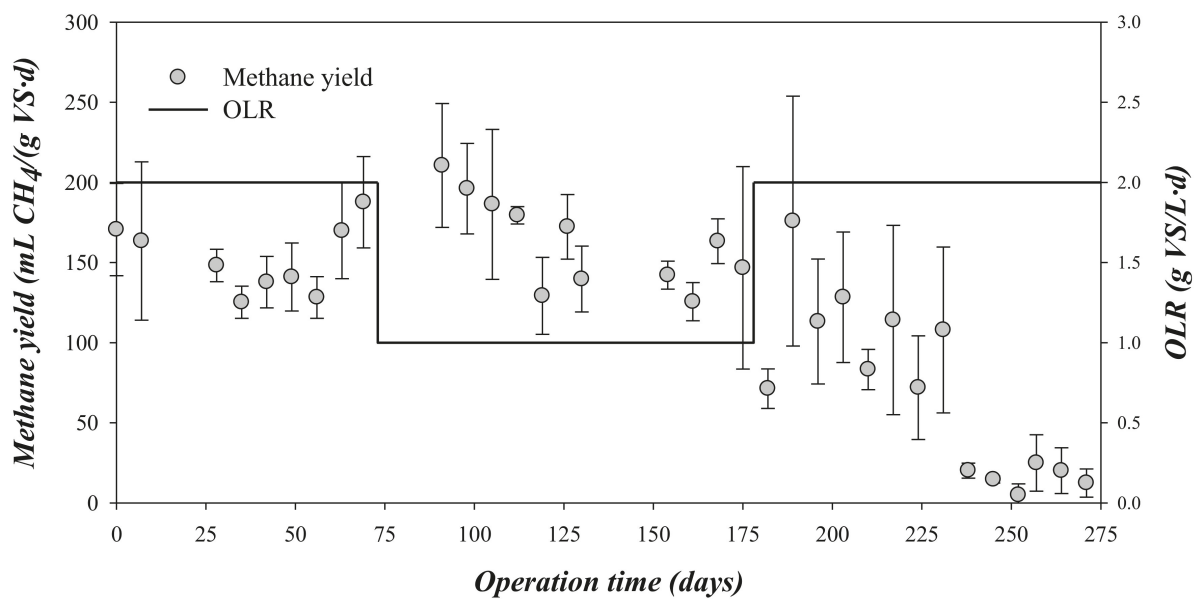

Figure 2. Variation of the methane yield and the organic loading rate (OLR) during the experimental time.

\subsection{Chemical Oxygen Demand and Volatile Fatty Acid Concentration throughout the Experimental Time}

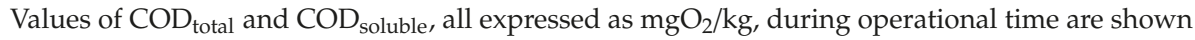
in Figure 3. During the first 175 days, i.e., stages 1 and 2, the concentration of $\mathrm{COD}_{\text {soluble }}$ remained at relatively constant values regardless of the applied OLR, with a mean value of $10,000 \mathrm{mgO} / \mathrm{kg}$. By contrast, the $\mathrm{COD}_{\text {total }}$ presented a clear increment from 12.0 to $27.0 \mathrm{gO}_{2} / \mathrm{kg}$ during the first stage, i.e., 0-75 days and OLR $=2 \mathrm{gVS} /(\mathrm{L} \cdot \mathrm{d})$. The accumulation of the $\mathrm{COD}_{\text {total }}$, when the $\mathrm{COD}_{\text {soluble }}$ and the VFA remained at low values, indicated that the hydrolysis was probably the limiting rate-step during this stage. In fact, the hydrolysis has been widely reported to be the limiting step in the anaerobic degradation pathway for complex organic solids [23]. The decrease of the OLR to $1 \mathrm{gVS} /(\mathrm{L} \cdot \mathrm{d})$, entailed a rapid decrease of the accumulated $\mathrm{COD}_{\text {total }}$ from days 75 to 100 . After this period, the concentration of $\mathrm{COD}_{\text {total }}$ remained at a mean value of $19.6 \pm 1.2 \mathrm{gO}_{2} / \mathrm{kg}$. 


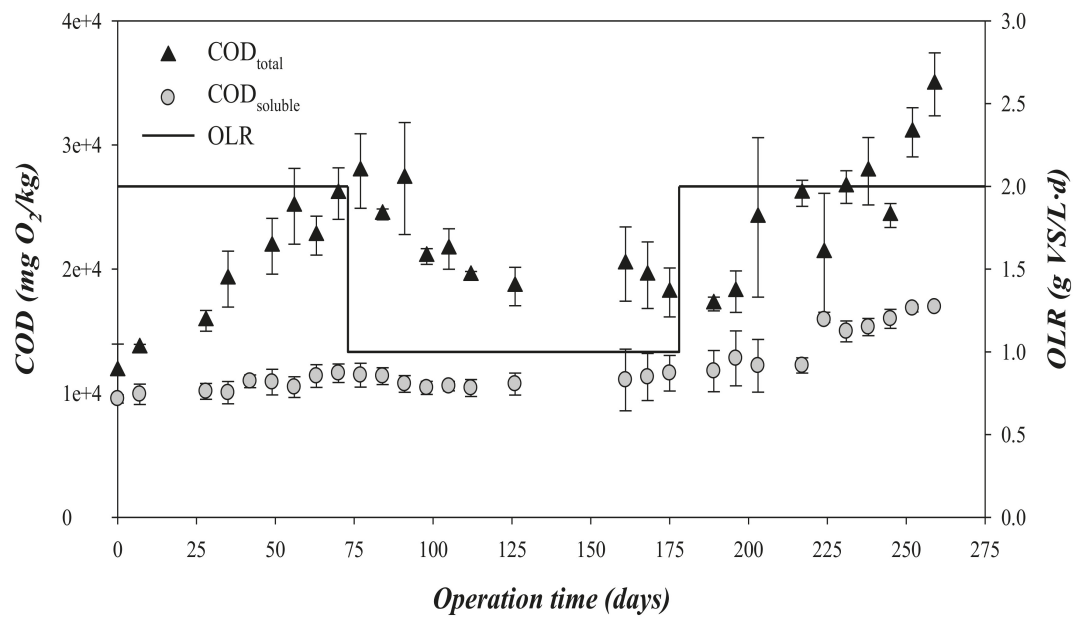

Figure 3. Variation of the total and soluble COD and the organic loading rate (OLR) during operation time.

The VFA composition was also analysed and the obtained results are shown in Figure 4. VFA concentration systematically decreased during the first stage (OLR $=2 \mathrm{gVS} /(\mathrm{L} \cdot \mathrm{d})$, days $0-75)$, from $\sim 3000 \mathrm{mgO}_{2} / \mathrm{kg}$ to $\sim 900 \mathrm{mgO}_{2} / \mathrm{kg}$, where propionic acid was the most abundant VFA determined in these first 75 days (Figure 4). VFA concentration remained almost undetectable during stage $2(\mathrm{OLR}=1 \mathrm{gVS} /(\mathrm{L} \cdot \mathrm{d})$, days 75-175). The decrease of propionic acid and almost disappearance of the rest of VFA during the second stage (75-175 days) carried out at an OLR of $1 \mathrm{gVS} /(\mathrm{L}$ d) could indicate that the microorganisms required this time period to complete their adaptation to the substrate [24]. Despite the initial increase in the VFA concentration, the $\mathrm{pH}$ of the digesters remained at mean values of $7.81 \pm 0.34$ and $7.39 \pm 0.41$ (Table 3), during stages 1 and 2, respectively. The $\mathrm{pH}$ remained slightly alkaline (above 7.8) until day 80 of operation. From that day, despite having reduced the OLR of the system, the alkalinity suffers a very marked decrease, which diminished from about $3220 \pm 100$ to $1460 \pm 400 \mathrm{mg} \mathrm{CaCO}_{3} / \mathrm{L}$. It should be noted that this substrate did not provide alkalinity. This decrease in the alkalinity was also associated with a decrease in $\mathrm{pH}$, from $7.9 \pm 0.1$ on day 75 to $7.0 \pm 0.2$ on day 175 .

After 175 days, the OLR once more increased up to $2 \mathrm{gVS} /(\mathrm{L} \cdot \mathrm{d})$. In view of the previously mentioned fall in alkalinity, alkalinity was added daily from day 175 to maintain the $\mathrm{pH}$ of the operation within the optimum values for anaerobic digestion. As a consequence, enormous oscillations in alkalinity values were observed in the third operating period (OLR: $2 \mathrm{gVS} /(\mathrm{L} \cdot \mathrm{d})$, days: 175-275). In spite of all the artificially added alkalinity, the $\mathrm{pH}$ during this stage falls repeatedly to values below those recommended, reaching values close to 6 . Although the acidification of the digesters was not reached until the end of this third stage, the acid $\mathrm{pH}$ and high acetic content of the OMSW (Table 1), i.e., $4.49 \pm 0.05$ and $1802 \pm 8 \mathrm{mg} / \mathrm{L}$, respectively, could acidify the process if the buffer capacities of the digesters were not high enough. The alkalinity values determined in the present research were markedly lower than that reported by Rincon et al. [25] for the anaerobic digestion of steam-exploded OMSW at mesophilic conditions. These authors reported alkalinity values in a range from 7000 to $9000 \mathrm{mg} \mathrm{CaCO} / \mathrm{L}$, which ensured that no acidification appeared despite the acid character of the OMSW. In the same way, Orive et al. [22] reported very high alkalinity values (10.6-21.3 $\left.\mathrm{g} \mathrm{CaCO}_{3} / \mathrm{L}\right)$ in digesters treating diluted OMSW, which allowed them to achieve $\mathrm{pH}$ values in the reactors within the optimal range for methanogenesis. 


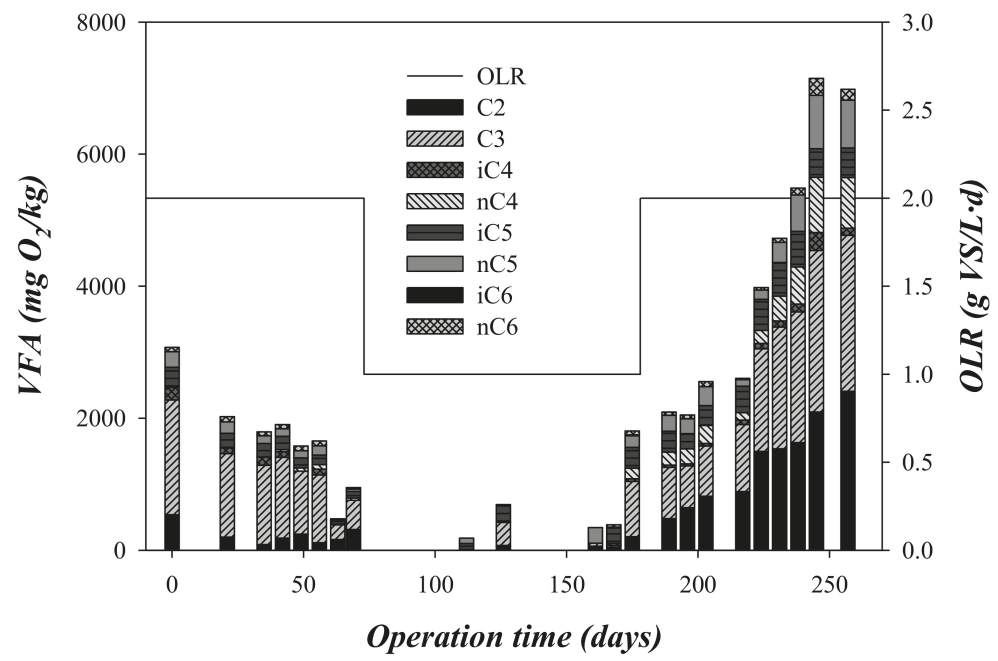

Figure 4. Variation of the VFA and the organic loading rate (OLR) with the operation time.

In this third stage, the $\mathrm{COD}_{\text {total }}$ critically increased above $28 \mathrm{gO}_{2} / \mathrm{kg}$ (Figure 3). $\mathrm{COD}_{\text {total }}$ accumulation was associated with a decrease in methane production, indicating a strong inhibition of the process (Figure 2). Process failure was also detected by $\mathrm{COD}_{\text {soluble }}$ and VFA accumulation during this stage (Figures 3 and 4). This accumulation indicated that the acidogenic and methanogenic microorganisms were inhibited prior to the hydrolytic microorganisms. This performance is in contrast to that observed from days 0 to 75 , where the concentrations of $\mathrm{COD}_{\text {soluble }}$ and VFA presented low values (Figures 2 and 3). The decrease in $\mathrm{pH}$ during the third phase due to the accumulation of VFA could explain the inhibition of the methanogenic activity [26]. As can be seen in Figure 4, the concentration of propionic acid increased during this third stage, being the most abundant VFA. Specifically, the mean propionic acid content increased from $251 \mathrm{mg} / \mathrm{L}$ during the second stage (OLR: $1 \mathrm{gVS} /(\mathrm{L} \cdot \mathrm{d})$ ) to $1486 \mathrm{mg} / \mathrm{L}$ in the third stage (OLR: $2 \mathrm{gVS} /(\mathrm{L} \cdot \mathrm{d})$ (Table 3). This accumulation was probably a consequence of the inhibition of the methanogens, which are the most sensitive microorganisms to acidification in the anaerobic processes [27]. The increment in the propionic acid concentration indicated that the inhibition also affected the microorganisms involved in the acetogenesis, and not only the methanogens. Although alkalinity was added periodically during the third stage, it was not able to compensate for the VFA accumulation, dropping the $\mathrm{pH}$ to values below 6 at the end of the third stage. Likewise, a recent study of anaerobic digestion of untreated OMSW in CSTR reactors carried out by Stoyanova et al. [12] revealed that the increase of the OLR up to a value of $1.76 \mathrm{gVS} /(\mathrm{L} \cdot \mathrm{d})$ entailed a drop in $\mathrm{pH}$ to values below 7. In the present study, the operation at an OLR of $2 \mathrm{gVS} /(\mathrm{L} \cdot \mathrm{d})$ was not stable, resulting in a strong inhibition due to the accumulation of solids in the digester, an increase of the VFA concentration and a marked drop of the $\mathrm{pH}$ and the alkalinity. However, at an OLR of $1 \mathrm{gVS} /(\mathrm{L} \cdot \mathrm{d})$, the effluent characterization confirmed the good performance of the digesters described for the high methane production rate obtained.

\subsection{Composition and Concentration of the Phenolic Compounds}

Phenolic compounds are reported to be toxic to anaerobic microorganisms, affecting the microbial growth [28]. In that sense, the presence of phenols, as total and soluble phenol concentration, and phenolic composition were monitored (Figure 5 and Table 4). The concentration of total and soluble phenolic compounds followed a similar trend to the values of $\mathrm{COD}_{\text {total }}$ (Figures 2 and 4). At an OLR $=2 \mathrm{gVS} /(\mathrm{L} \cdot \mathrm{d})$, the phenolic compounds accumulated in the digesters, whereas at an OLR of $1 \mathrm{gVS} /(\mathrm{L} \cdot \mathrm{d})$, the phenol concentration was kept at a lower constant concentration (Figure 5) compared 
to an OLR of $2 \mathrm{gVS} /(\mathrm{L} \cdot \mathrm{d})$. The relation between soluble phenol and total phenol was close to 1 during stages 1 and 2 . This value is much higher than the ratio of soluble phenol/total phenol in the substrate, which presented a value of 0.31 (Table 1). This difference indicated that the phenols were partially hydrolyzed during the biomethanization. However, the presence in the digesters of detectable concentrations of vanillin and 4-ethylphenol indicated that the degradation was not complete (Table 4) [3].

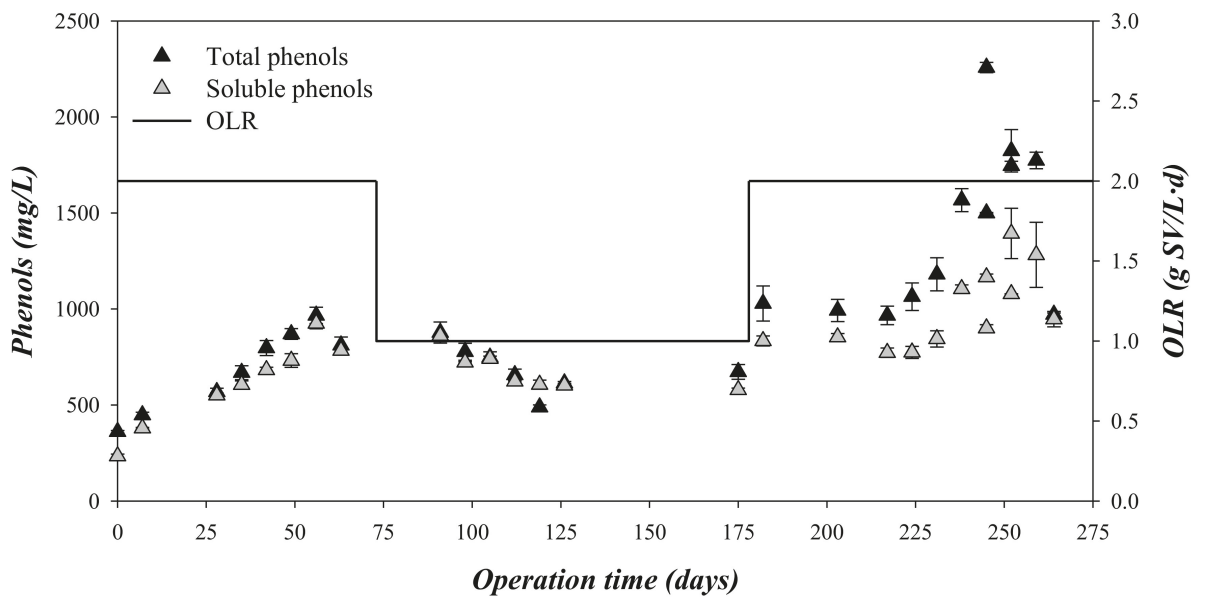

Figure 5. Variation in the total and soluble phenols and the organic loading rate (OLR) with the operation time.

Table 4. Individual phenolic compound concentration during the different organic loading rates (OLRs), where n.d., non detected; Traces, $<0.01 \mathrm{mg} / \mathrm{L}$.

\begin{tabular}{ccccc}
\hline Experimental Stage & Start & End Stage 1 & End Stage 2 & End Stage 3 \\
\hline Day & $\mathbf{0}$ & $\mathbf{6 3}$ & $\mathbf{1 7 5}$ & $\mathbf{2 7 5}$ \\
\hline OLR (gVS/(L*d)) & - & $\mathbf{2}$ & $\mathbf{1}$ & $\mathbf{2}$ \\
\hline 3,4-Dihydroxyphenylglycol & $0.35 \pm 0.04$ & $0.08 \pm 0.01$ & Traces & $0.62 \pm 0.05$ \\
Hydroxymethylfurfural & n.d. & n.d. & n.d. & n.d. \\
Hydroxytyrosol & $3.90 \pm 0.17$ & $3.49 \pm 0.05$ & $3.87 \pm 0.55$ & $10.3 \pm 2.52$ \\
Tyrosol & n.d. & n.d. & n.d. & $9.9 \pm 0.4$ \\
Homovanillic acid & n.d. & n.d. & n.d. & n.d. \\
Vanillic acid & n.d. & n.d. & n.d. & $1.3 \pm 0.4$ \\
Vanillin & $48.1 \pm 6.9$ & $30.8 \pm 11.8$ & $25.6 \pm 2.1$ & $149 \pm 55$ \\
4-Ethylphenol & $23.2 \pm 2.9$ & $141 \pm 33$ & $108 \pm 6$ & $136 \pm 15$ \\
\hline
\end{tabular}

Regardless of the OLR applied to the digester, the phenol and furanic concentration was always lower than the limits described as inhibitory for the anaerobic digestion. For example, hydroxymethylfurfural was totally biodegraded, and, therefore, was not detected, whereas vanillin concentrations were lower than $150 \mathrm{mg} / \mathrm{L}$ throughout the experimental time (Table 3). The inhibition concentrations for anaerobic processes were reported to be around $800 \mathrm{mg} / \mathrm{L}$ for hydroxymethylfurfural and $2000 \mathrm{mg} / \mathrm{L}$ for vanillin [16]. Both compounds, i.e., hydroxymethylfurfural and vanillin, have been widely defined as the most toxic phenolic and furanic compounds for biomethanization $[3,16,29]$. Therefore, the failure of the biomethanization process determined at an OLR of $2 \mathrm{gVS} /(\mathrm{L} \cdot \mathrm{d})$ was probably a consequence of overloading of the system, with acidification of the process, instead of the toxic effect of the accumulated phenolic and furanic compounds [30]. 


\subsection{Microbiology Assessment}

On the last day of each tested OLR, the number of microbes was determined from fluorescence images in the inoculum and at 75, 175, and 250 days after starting the experiment (Figure 6). The mean number of microorganisms decreased from $12,761 \pm 3213$ cells $/ \mathrm{mm}^{2}$ in the inoculum to $7126 \pm 3099$ cells $/ \mathrm{mm}^{2}$ after 75 days (Figure 7) as a result of a process of selection by the substrate [31] The number of microbes was no different at day 175 (5739 \pm 1316 cells $\left./ \mathrm{mm}^{2}\right)$ from day 75, which suggests that the rate of duplication met an equilibrium with the OLR. Interestingly, duplication rate was similar to the production of methane, which suggest that most cells are methanogenic bacteria. Accordingly, the number of microbes dramatically decreased at day $250\left(2159 \pm 801 \mathrm{cells} / \mathrm{mm}^{2}\right)$ where a low production of methane was detected.
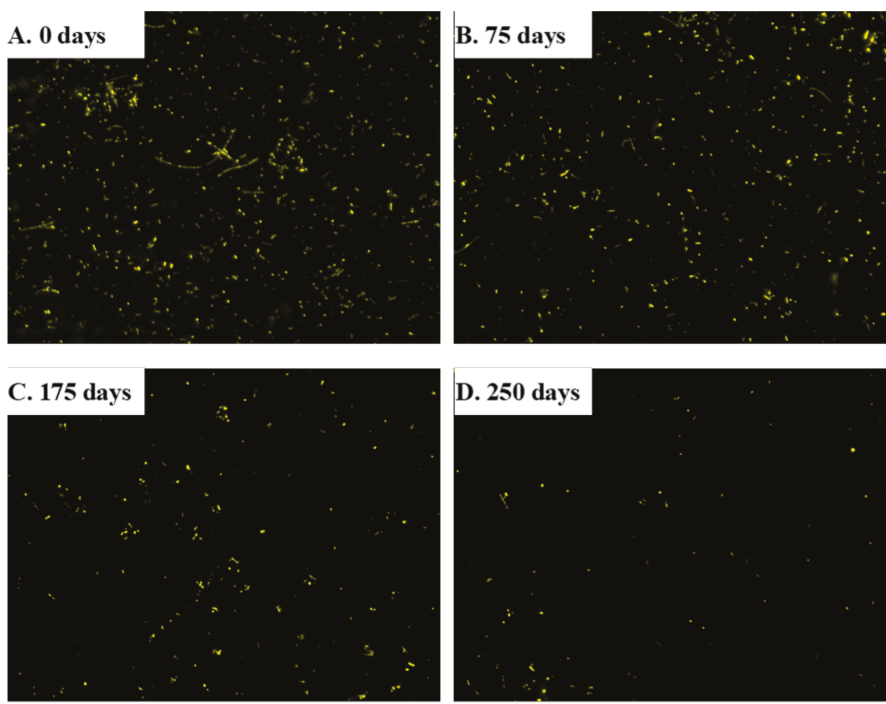

Figure 6. DAPI stained images for the determination of bacterial abundance at distinct experimental times.

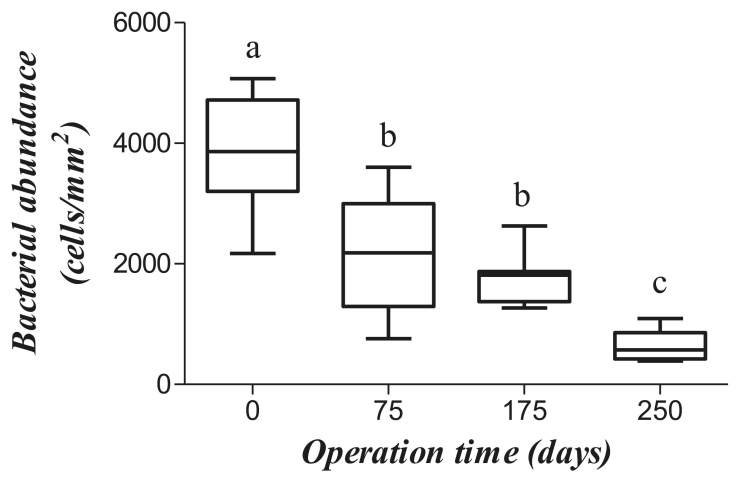

Figure 7. Bacterial abundance at distinct operation times. Boxes with distinct letters are significantly different ( $p<0.05$, one-way ANOVA plus Tukey post-hoc test). 


\subsection{Economic Assessment}

The results obtained for the profitability assessment are summarised in Table 5. The proposed bio-refinery concept (Figure 1) shows high values for both NPV (3.9.10 $\mathrm{M} €$ ) and IRR (10 11\%), and a short payback period (1 year), which point to high profitability. Regarding the influence of the price of the phenol extract, the project would be profitable for prices higher than $42.2 € / \mathrm{kg}$. This minimum price is almost lower than the minimum price of $51.8 € / \mathrm{kg}$ obtained for a similar bio-refinery concept in which the OMSW is pre-treated with steam at $170{ }^{\circ} \mathrm{C}[13]$.

Table 5. Economical assessment.

\begin{tabular}{|c|c|c|c|c|c|c|c|}
\hline $\begin{array}{l}\text { Methane } \\
\text { Production } \\
\text { (Nm³/year) }\end{array}$ & $\begin{array}{c}\text { Initial } \\
\text { Investment } \\
\text { Cost }(€)\end{array}$ & $\begin{array}{l}\text { Incoming } \\
\text { (€/year) }\end{array}$ & $\begin{array}{l}\text { Annual } \\
\text { Costs } \\
(€ / \text { year })^{a}\end{array}$ & $\begin{array}{l}\text { NPV } \\
\text { (M€) }\end{array}$ & IRR (\%) & $\begin{array}{l}\text { Payback } \\
\text { Period } \\
\text { (years) }\end{array}$ & $\begin{array}{l}\text { Limit Phenol } \\
\text { Price }(€ / \mathrm{kg})^{b}\end{array}$ \\
\hline $2,951,115$ & $28,367,480$ & $330,066,600$ & $28,277,122$ & $3.9 \cdot 10^{3}$ & $10^{11}$ & 1 & 42.2 \\
\hline
\end{tabular}

\section{Conclusions}

The anaerobic digestion process was stable at an OLR of $1 \mathrm{gVS} /(\mathrm{L} \cdot \mathrm{d})$, with a specific production rate of $163 \pm 28 \mathrm{~mL} \mathrm{CH}_{4} /(\mathrm{gVS} \cdot \mathrm{d})$. However, the increment of the OLR up to $2 \mathrm{gVS} /(\mathrm{L} \cdot \mathrm{d})$ resulted in total exhaust of the methane production. The increment in the total and soluble organic matter, especially the increment in the propionic acid concentration up to $1486 \mathrm{mg} / \mathrm{L}$, described an overloaded inhibition process. Regardless of the OLR, the concentration of phenolic compounds was always lower than the inhibition limits. An economic analysis showed that the combination of the steam-explosion recovery of phenols and the anaerobic digestion, under stable conditions, could generate high profitability, which would still be profitable for a phenol extract price higher than $42.2 € / \mathrm{kg}$. Therefore, steam-exploited OMSW could be a suitable substrate for anaerobic digestion at the correct OLR.

Author Contributions: Conceptualization: A.S., F.G.F., B.A.-F., G.R.-G., S.L., J.F.-B. and R.B.; Methodology: A.S., F.G.F., B.A.-F., G.R.-G., S.L., J.F.-B. and R.B.; Validation: A.S., F.G.F., B.A.-F., G.R.-G., S.L., J.F.-B. and R.B.; Formal Analysis, A.S., F.G.F., B.A.-F., G.R.-G., S.L., J.F.-B. and R.B.; Investigation: A.S., F.G.F., B.A.-F., G.R.-G., S.L., J.F.-B. and R.B.; Resources: A.S., F.G.F., B.A.-F., G.R.-G., S.L., J.F.-B. and R.B.; Data Curation: A.S., F.G.F., B.A.-F., G.R.-G., S.L., J.F.-B. and R.B.; Writing-Original Draft Preparation: A.S., F.G.F., B.A.-F., G.R.-G., S.L., J.F.-B. and R.B.; Writing-Review \& Editing: A.S., F.G.F., B.A.-F., G.R.-G., S.L., J.F.-B. and R.B.; Supervision: F.G.F. and R.B.; Project Administration: F.G.F. and R.B.; Funding Acquisition: F.G.F. and R.B.

Funding: This research was funded by the [Spanish Ministry of Economy and Competitiveness] through Project grant number CTM2014-55095-R and the Ramon y Cajal Programme (RyC 2012-10456). Sergio López acknowledges the financial support from the 'V Own Research Plan' of the University of Seville (VPPI-US) for his research contract. This contracts is cofunded by the European Social Fund.

Acknowledgments: The authors wish to express their gratitude to. Ainoa Botana for her assistance to this research.

Conflicts of Interest: The authors declare no conflict of interest.

\section{References}

1. Mahmoud, A.E.; Fathy, S.A.; Ali, M.M.; Ezz, M.K.; Mohammed, A.T. Antioxidant and anticancer efficacy of therapeutic bioactive compounds from fermented olive waste. Grasas y Aceites 2018, 69, 266. [CrossRef]

2. Tortosa, G.; Alburquerque, J.A.; Bedmar, E.J.; Ait-Baddi, G.; Cegarra, J. Strategies to produce commercial liquid organic fertilisers from "alperujo" composts. J. Clean. Prod. 2014, 82, 37-44. [CrossRef]

3. Serrano, A.; Fermoso, F.G.; Alonso-Fariñas, B.; Rodríguez-Gutierrez, G.; Fernandez-Bolaños, J.; Borja, R. Olive mill solid waste biorefinery: High-temperature thermal pre-treatment for phenol recovery and biomethanization. J. Clean. Prod. 2017, 148, 314-323. [CrossRef]

4. Rodríguez-Gutiérrez, G.; Lama-Muñoz, A.; Rubio-Senent, F.; Fernández-Bolaños, J.; Ruiz-Méndez, M.V. New Olive-Pomace Oil Improved by Hydrothermal Pre-Treatments; INTECH Open Access Publisher: London, UK, 2012. 
5. García, A.; Rodríguez-Juan, E.; Rodríguez-Gutiérrez, G.; Rios, J.J.; Fernández-Bolaños, J. Extraction of phenolic compounds from virgin olive oil by deep eutectic solvents (DESs). Food Chem. 2016, 197, 554-561. [CrossRef] [PubMed]

6. Rodríguez, G.; Lama, A.; Rodríguez, R.; Jiménez, A.; Guillén, R.; Fernández-Bolaños, J. Olive stone an attractive source of bioactive and valuable compounds. Bioresour. Technol. 2008, 99, 5261-5269. [CrossRef] [PubMed]

7. Rubio-Senent, F.; Rodríguez-Gutíerrez, G.; Lama-Muñoz, A.; Fernández-Bolaños, J. New phenolic compounds hydrothermally extracted from the olive oil byproduct alperujo and their antioxidative activities. J. Agric. Food Chem. 2012, 60, 1175-1186. [CrossRef] [PubMed]

8. Hendriks, A.T.W.M.; Zeeman, G. Pretreatments to enhance the digestibility of lignocellulosic biomass. Bioresour. Technol. 2009, 100, 10-18. [CrossRef]

9. Serrano, A.; Fermoso, F.G.; Alonso-Fariñas, B.; Rodríguez-Gutierrez, G.; Fernandez-Bolaños, J.; Borja, R. Phenols recovery after steam explosion of Olive Mill Solid Waste and its influence on a subsequent biomethanization process. Bioresour. Technol. 2017, 243, 169-178. [CrossRef]

10. Christoforou, E.; Fokaides, P.A. A review of olive mill solid wastes to energy utilization techniques. Waste Manag. 2016, 49, 346-363. [CrossRef]

11. Al-Mallahi, J.; Furuichi, T.; Ishii, K. Appropriate conditions for applying NaOH-pretreated two-phase olive milling waste for codigestion with food waste to enhance biogas production. Waste Manag. 2016, 48 (Suppl. C), 430-439. [CrossRef]

12. Stoyanova, E.; Lundaa, T.; Bochmann, G.; Fuchs, W. Overcoming the bottlenecks of anaerobic digestion of olive mill solid waste by two-stage fermentation. Environ. Technol. 2017, 38, 394-405. [CrossRef] [PubMed]

13. Serrano, A.; Fermoso, F.G.; Alonso-Fariñas, B.; Rodríguez-Gutiérrez, G.; López, S.; Fernandez-Bolaños, J.; Borja, R. Performance evaluation of mesophilic semi-continuous anaerobic digestion of high-temperature thermally pre-treated olive mill solid waste. Waste Manag. 2019, 87, 250-257. [CrossRef] [PubMed]

14. Borja, R.; Alba, J.; Banks, C.J. Impact of the main phenolic compounds of olive mill wastewater (OMW) on the kinetics of acetoclastic methanogenesis. Process Biochem. 1997, 32, 121-133. [CrossRef]

15. Kourmentza, C.; Koutra, E.; Venetsaneas, N.; Kornaros, M. Integrated Biorefinery Approach for the Valorization of Olive Mill Waste Streams towards Sustainable Biofuels and Bio-Based Products. In Microbial Applications Vol.1: Bioremediation and Bioenergy; Kalia, V.C., Kumar, P., Eds.; Springer International Publishing: Cham, Switzerland, 2017; pp. 211-238.

16. Ghasimi, D.S.M.; Aboudi, K.; de Kreuk, M.; Zandvoort, M.H.; van Lier, J.B. Impact of lignocellulosic-waste intermediates on hydrolysis and methanogenesis under thermophilic and mesophilic conditions. Chem, Eng, J. 2016, 295, 181-191. [CrossRef]

17. American Public Health Association, American Water Works Association, The Water Environment Federation. Standard Methods for the Examination of Water and Wastewater; APHA-AWWA-WEF: Washington, DC, USA, 2005.

18. Siritantikorn, S.; Jintaworn, S.; Noisakran, S.; Suputtamongkol, Y.; Paris, D.H.; Blacksell, S.D. Application of ImageJ program to the enumeration of Orientia tsutsugamushi organisms cultured in vitro. Trans. R. Soc. Trop. Med. Hyg. 2012, 106, 632-635. [CrossRef] [PubMed]

19. Ciriminna, R.; Meneguzzo, F.; Fidalgo, A.; Ilharco, L.M.; Pagliaro, M. Extraction, benefits and valorization of olive polyphenols. Eur. J. Lipid Sci. Technol. 2016, 118, 503-511. [CrossRef]

20. EUROSTAT. Available online: https://ec.europa.eu/eurostat/web/main/home (accessed on 27 March 2019).

21. endesaonline.es. Available online: www.endesaonline.es (accessed on 20 March 2019).

22. Orive, M.; Cebrián, M.; Zufía, J. Techno-economic anaerobic co-digestion feasibility study for two-phase olive oil mill pomace and pig slurry. Renew. Energy 2016, 97, 532-540. [CrossRef]

23. Ortega, L.; Husser, C.; Barrington, S.; Guiot, S.R. Evaluating limiting steps of anaerobic degradation of food waste based on methane production tests. Water Sci. Technol. 2008, 57, 419-422. [CrossRef]

24. Wang, Y.; Zhang, Y.; Wang, J.; Meng, L. Effects of volatile fatty acid concentrations on methane yield and methanogenic bacteria. Biomass Bioenergy 2009, 33, 848-853. [CrossRef]

25. Rincón, B.; Rodríguez-Gutiérrez, G.; Bujalance, L.; Fernández-Bolaños, J.; Borja, R. Influence of a steam-explosion pre-treatment on the methane yield and kinetics of anaerobic digestion of two-phase olive mil solid waste or alperujo. Process Saf. Environ. 2016, 102, 361-369. [CrossRef]

26. Liu, C.; Yuan, X.; Zeng, G.; Li, W.; Li, J. Prediction of methane yield at optimum pH for anaerobic digestion of organic fraction of municipal solid waste. Bioresour. Technol. 2008, 99, 882-888. [CrossRef] [PubMed] 
27. Borja, R. Biogas Production. In Comprehensive Biotechnology, 2nd. ed; Elsevier: Amsterdam, The Netherlands, 2011; Volume 2, pp. 785-798.

28. Battista, F.; Fino, D.; Erriquens, F.; Mancini, G.; Ruggeri, B. Scaled-up experimental biogas production from two agro-food waste mixtures having high inhibitory compound concentrations. Renew. Energy 2015, 81 (Suppl. C), 71-77. [CrossRef]

29. Carrere, H.; Antonopoulou, G.; Affes, R.; Passos, F.; Battimelli, A.; Lyberatos, G.; Ferrer, I. Review of feedstock pretreatment strategies for improved anaerobic digestion: From lab-scale research to full-scale application. Bioresour. Technol. 2016, 199, 386-397. [CrossRef]

30. Theuerl, S.; Klang, J.; Prochnow, A. Process disturbances in agricultural biogas production-Causes, mechanisms and effects on the biogas microbiome: A review. Energies 2019, 12, 365. [CrossRef]

31. Alcántara-Hernández, R.J.; Taş, N.; Carlos-Pinedo, S.; Durán-Moreno, A.; Falcón, L.I. Microbial dynamics in anaerobic digestion reactors for treating organic urban residues during the start-up process. Lett. Appl. Microbiol. 2017, 64, 438-445. [CrossRef] [PubMed]

(C) 2019 by the authors. Licensee MDPI, Basel, Switzerland. This article is an open access article distributed under the terms and conditions of the Creative Commons Attribution (CC BY) license (http://creativecommons.org/licenses/by/4.0/). 

MDPI

St. Alban-Anlage 66

4052 Basel

Switzerland

Tel. +41616837734

Fax +41 613028918

www.mdpi.com

Energies Editorial Office

E-mail: energies@mdpi.com www.mdpi.com/journal/energies

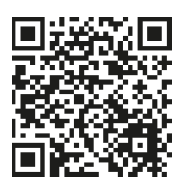



MDPI

St. Alban-Anlage 66

4052 Basel

Switzerland

Tel: +41 616837734

Fax: +41 613028918 